\title{
SYNTHESIS, MICROSCOPY AND MANIPULATION OF COLLOIDAL MATTER
}



Synthesis, microscopy and manipulation of colloidal matter 
Cover: A test tube coated with a colloidal crystal of silica particles illuminated with white light via a pinhole in a blank canvas. The bright colours result from Bragg interference of light reflected from different planes of the regular structure. A micrograph showing the individual particles may be found in Figure $2.12 \mathrm{c}$.

PhD thesis, Utrecht University, the Netherlands, 2020.

ISBN: $978-90-393-7330-9$

DOI: $10.33540 / 132$

A digital version of this thesis is available in the repository of Utrecht University. 


\section{Synthesis, microscopy and manipulation of colloidal matter}

\section{Synthese, microscopie en manipulatie van colloïdale materie}

(met een samenvatting in het Nederlands)

\section{Proefschrift}

ter verkrijging van de graad van doctor aan de Universiteit Utrecht op gezag van de rector magnificus, prof. dr. H.R.B.M. Kummeling, ingevolge het besluit van het college voor promoties in het openbaar te verdedigen op woensdag 25 november 2020 des ochtends te 9.15 uur

$$
\text { door }
$$

\section{Chris Lee Kennedy}

geboren op 20 februari 1993 te Glasgow, Schotland 
Promotor: $\quad$ Prof. dr. A. van Blaaderen

This work was supported by the Netherlands Center for Multiscale Catalytic Energy Conversion (MCEC), an NwO Gravitation programme funded by the Ministry of Education, Culture and Science of the government of the Netherlands. 


\section{Contents}

1 Introduction 1

1.1 Hierarchy . . . . . . . . . . . . . . . . . . . 2

1.2 The colloidal domain . . . . . . . . . . . . . . . . . 4

1.3 The Toolkit .................... . . 5

1.3.1 Creation: Colloidal synthesis . . . . . . . . . 5

1.3.2 Observation: Microscopy . . . . . . . . . . . . . 6

1.3.3 Manipulation: Phoresis . . . . . . . . . . . . . . 6

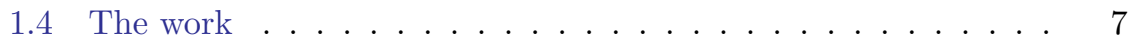

2 Synthesis of monodisperse fluorescent silica nanospheres 9

2.1 Introduction . . . . . . . . . . . . . . . . 10

2.2 Theory . . . . . . . . . . . . . . . . . . . 14

2.2.1 Particle formation and growth in the amino acid catalysed method . . . . . . . . . . . . . . . 14

2.2.2 Control parameters in the two phase method . . . . . . 14

2.2 .3 Stöber Silica . . . . . . . . . . . . . . . . . . 16

2.2.4 Polydispersity in seeded growth . . . . . . . . . . . 17

2.3 Methods . . . . . . . . . . . . . . . . . . . . . 18

2.3 .1 Materials . . . . . . . . . . . . . . . . . 18

2.3 .2 Notation. . . . . . . . . . . . . . . . . . . . . . 19

2.3.3 Aqueous Seed Synthesis . . . . . . . . . . . . . . . . 19

2.3.4 Direct growth . . . . . . . . . . . . . . . . . . 20

2.3.5 Diluted arginine growth . . . . . . . . . . . . 20

2.3 .6 Stöber growth . . . . . . . . . . . . . . . 21

2.3.7 Surface modification . . . . . . . . . . . . . . . . . . 24

2.3.8 Particle characterisation . . . . . . . . . . . . . 25

2.3.9 Optical Microscopy . . . . . . . . . . . . . . . . . . 25

2.4 Results and Discussion . . . . . . . . . . . . . . . . . 26

2.4 Seed Synthesis . . . . . . . . . . . . . . . . 26

2.4.2 Direct arginine growth . . . . . . . . . . . . . . . 28

2.4 .3 Diluted arginine growth . . . . . . . . . . . . . . 29

2.4 .4 Immediate Stöber growth . . . . . . . . . . . . . . 31

2.4.5 Early (dyed) Stöber growth . . . . . . . . . . . . . 34

2.4.6 Late Stöber growth . . . . . . . . . . . . . . . . . 37 
2.4.7 Decreasing polydispersity upon seeded growth . . . . . . 37

2.4.8 Surface Treatment . . . . . . . . . . . . . . . 41

2.4.9 Efficacy of the dye in different systems . . . . . . . . . . 42

2.5 Conclusions ..................... 45

2.6 Outlook ........................ 46

3 The Nanopede: Parallelised microfluidics for colloidal droplets $\quad 47$

3.1 Introduction . . . . . . . . . . . . . . . . . . . . 48

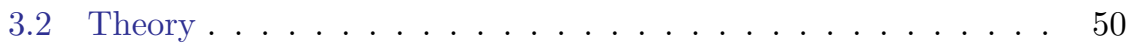

3.2.1 Droplet Formation . . . . . . . . . . . . . . 50

3.2.2 Droplet Size . . . . . . . . . . . . . . . . 52

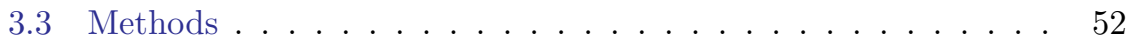

3.3.1 The Nanopede . . . . . . . . . . . . 52

3.3.2 General emulsification procedure .......... 53

3.3.3 Hexadecane Droplets . . . . . . . . . . . . . . . . 53

3.3.4 Polymer Particles . . . . . . . . . . . . . . . . 56

3.3.5 Supraparticles . . . . . . . . . . . . . 56

3.3.6 Operational Notes . . . . . . . . . . . . . . 57

3.4 Results and Discussion . . . . . . . . . . . . . . . . 59

3.4.1 Monodisperse 1 um droplets . . . . . . . . . . . 59

3.4.2 Imaging droplet formation . . . . . . . . . . . . . . . . . 64

3.4.3 Supraparticles .................... 65

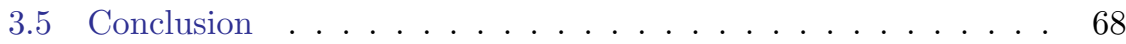

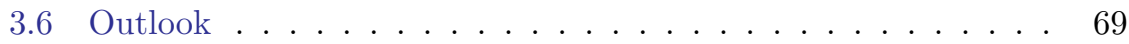

3.A Method to collect a volatile creaming emulsion produced in a microfluidic device . . . . . . . . . . . . . 70

4 Deep STED microscopy: experimental PSFs and deconvolution $\mathbf{7 3}$

4.1 Introduction . . . . . . . . . . . . . . . . 74

4.2 Theory............................... 77

4.2.1 Image formation . . . . . . . . . . . . . . . . 77

4.2 .2 Deconvolution ................... 78

4.3 Methods ..................... . . 82

4.3.1 Sample Preparation . . . . . . . . . . . . 82

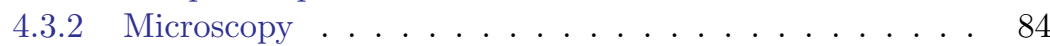

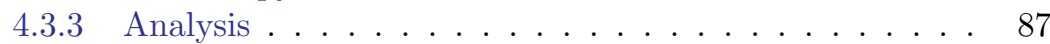

4.4 Results and Discussion . . . . . . . . . . . . . . . . . 88

4.4.1 Behaviour of the depletion beam far from the objective 88

4.4 The measured PSF . . . . . . . . . . . . . 93

4.4.3 Depth Variant Deconvolution . . . . . . . . . . . . 97

4.4.4 Deconvolution of synthetic images . . . . . . . . . 108 
4.5 Conclusions . . . . . . . . . . . . . . . . . . . 108

4.6 Outlook ............................. 110

4.A Linear focal shift between excitation and depletion spots . . . . 111

5 Diffusiophoresis at the single particle level $\mathbf{1 1 5}$

5.1 Introduction . . . . . . . . . . . . . . . . . . . 116

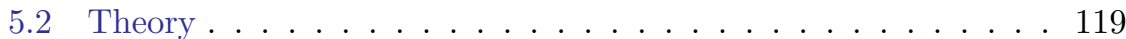

5.2.1 Diffusiophoresis . . . . . . . . . . . . . . 119

5.2.2 Zeta potential measurement with electrophoresis . . . . 123

5.2.3 Salt gradient evolution along the channel . . . . . . . . 124

5.3 Methods . . . . . . . . . . . . . . . . 126

5.3.1 Particle synthesis and characterisation . . . . . . . 126

5.3.2 Microfluidic Experiments . . . . . . . . . . . 126

5.3.3 Microscopy and image analysis . . . . . . . . . . . 127

5.3.4 Fluorescent dye diffusion . . . . . . . . . . . . . 128

5.4 Results and Discussion . . . . . . . . . . . . . . . . . 128

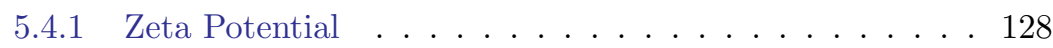

5.4 Diffusiophoresis of the ensemble . . . . . . . . . . 129

5.4.3 Diffusiophoresis of single particles . . . . . . . . . 132

5.4 .4 The salt gradient . . . . . . . . . . . . . . . . 136

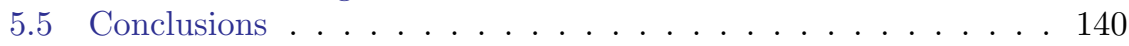

5.6 Outlook . . . . . . . . . . . . . . . . . . 140

5.A Diffusiophoresis of TPM particles . . . . . . . . . . . . . 142

$\begin{array}{ll}\text { Bibliography } & 143\end{array}$

$\begin{array}{ll}\text { Summary for a broad audience } & 161\end{array}$

$\begin{array}{ll}\text { Samenvatting voor een breed publiek } & 165\end{array}$

$\begin{array}{lr}\text { Acknowledgements } & 169\end{array}$

$\begin{array}{ll}\text { About the author } & 171\end{array}$ 



\title{
1 Introduction
}

\begin{abstract}
The subjects of the thesis are placed in broad context and the relevant concepts articulated in an informal manner. This style is chosen to facilitate insight and intuition before the physical phenomena are more formally introduced in the following chapters. Provision of historical context is likewise delegated to the separate chapters, while this general introduction is presented in a logical order from a modern perspective.
\end{abstract}




\section{Introduction}

\subsection{Hierarchy}

It is not possible to hold in one's human mind an idea that contains the entire universe as a single holistic concept. To make some sense of it all, we divide it up into smaller objects and ideas and think about how they behave and interact with each other. These pieces might be anything from a grain of sand to a flock of sheep to a galaxy, anything to avoid thinking about everything at once! Of course we can do better than just dividing things up, we should also organise them. Having a concept of a grain of sand is all very well, as is having the idea of a beach, but better yet is to understand how the two are related. Namely that one is a collection of many separate entities of the other. This is a useful hierarchy: we needn't worry about the individual grains every time we wish to walk on the beach, but it is convenient to know what a small part of the beach looks like if we accidentally take it home between our toes.

Structural hierarchies such as these, where something is made up of multiple subunits, are everywhere. We have covered the beach, but also our flock of sheep is made up of individual animals. Each of those is composed of several million much smaller cells, and so on all the way down to the subatomic particles which are fundamental as far as we know.

The complexity of the hierarchy is highly variable. While most matter that we interact with has atoms as a common building block, above those the possibilities are innumerable. For example, let us consider the differences between a cast iron stove and a hearth made of bricks and mortar. The atoms in cast iron are strongly bonded together and can be arranged in various ways. Zooming out a little, it becomes clear that the material has not only one type of atomic arrangement, but contains a patchwork of domains with atoms organised differently in adjacent patches. The sizes and structures of these domains depend on the way the iron object was made and determine many of its properties.

For the hearth on the other hand, we can step down through some extra structural levels before again arriving at the atoms that the two have in common $^{1}$. The first and most obvious step is that from the walls of the fireplace to the individual bricks comprising them. A more interesting rung on the ladder is below that, as we cannot yet say that the brick is domains of atoms from here on down.

Zooming in on the brick to a scale just below that accessible with the naked eye, domains will appear but we should keep an eye on our magnification

\footnotetext{
${ }^{1}$ This approach to the topic was inspired by a discussion in Reference 1 , in which the hierarchy of a forest is the illustrative example. The aforementioned book is recommended to everyone: from the layperson looking for an introduction to colloid science to the expert who will frequently benefit from novel perspectives therein.
} 
because these domains are not made of atoms, but something hundreds or thousands of times larger. These are the clay particles which the brick itself is built from [2]. Within each particle, atomic domains and interatomic bonds are found, thus reconciling with the case of cast iron. The clay particles can have different arrangements and form domains just like the atoms.

What is the difference between these 'particles' and arbitrary subunits of cast iron? In wet clay, these particles are able to slide past each other and the mass may be moulded into different shapes. This is because there are not strong interatomic bonds linking two clay particles, but weaker and longer ranged forces which are discussed in the following section. Only with drying and firing at high temperature do the edges of the discrete particles melt together and from bridges of bonded atoms upon cooling [3]. The particles only stick at certain points of contact, so the result is a porous network with a complex structure, quite distinct from the 'landlocked' domains in cast iron.

The structural hierarchies may be summarised as follows for cast iron:

$$
\text { atoms } \rightarrow \text { atomic domains } \rightarrow \text { cast iron } \rightarrow \text { stove }
$$

and brick:

$$
\begin{aligned}
& \text { atoms } \rightarrow \text { atomic domains } \rightarrow \text { clay mineral particles } \rightarrow \text { particle domains } \rightarrow \\
& \text { bricks } \rightarrow \text { brick wall } \rightarrow \text { hearth. }
\end{aligned}
$$

Knowledge of the microstructure allows us to answer many questions about our fireplace materials which cannot be explained simply from consideration of the chemical components. Why does an iron bar weigh so much more than a brick of the same size? Why does the brick have much lower heat conductivity? Why is the brick able to absorb so much water while the iron bar cannot? The answers to all of these questions lie in the pores of the brick, which may be full of lightweight and insulating air or with water sponged from the environment.

We have seen that insight may be gained from consideration of the individual bricks which were used to build the wall, and now turn our attention to the individual particles which were used to build the brick. To get there, let us return to the brick-making process, specifically to the wet clay. If we go in the opposite direction to pottery and brick-making, and keep adding water, we will make a thick slurry and eventually: muddy water. A familiar feature of the latter is that when your feet are immersed in it, they cannot be seen through the liquid. This is a good indication that the clay is not dissolved in the water (in contrast to when salt is added to water, and it becomes transparent) but is dispersed in it as particles much larger than atoms, which scatter the light between your feet and your eyes.

A system of discrete particles such as this is known as a colloidal dispersion. To omit the colloidal size region in our thinking by skipping all the way from 


\section{Introduction}

macroscopic objects to atoms would leave many phenomena unexplainable. Principal among these are processes occurring in the most complex hierarchy we know of: human life.

\subsection{The colloidal domain}

The boundaries of the colloidal size range are not rigidly defined, with the broadest definition including particles with at least one dimension in the range $1 \mathrm{~nm}-10 \mu \mathrm{m}$ [4]. Below this range, the dispersion approaches the behaviour of a true solution, and above it lies the realm of granular materials [5]. The change which justifies having an upper limit is that of the relative importance of the thermal energy of the particle (which does not change with size) to its behaviour. For a $1 \mathrm{~nm}$ particle, there is significant thermal motion at room temperature, while this is negligible for a grain of sand, so the latter is considered granular and not colloidal. The boundary is fuzzy because the degree of thermal motion depends on the material properties and because the definition of 'significant motion' depends on how long a human experimenter is willing to wait for it. Therefore the upper limit may fall anywhere between 1 and $10 \mu \mathrm{m}$.

The surface to volume ratio of solid objects increases with decreasing size. This means that the surface properties of colloidal particles greatly influence their behaviour, while for macroscopic objects, the properties of the bulk often dominate. In fact, colloidal stability (the property of staying as discrete particles and not sticking together) is due entirely to surface phenomena. With only volume effects, all similar colloidal particles would irreversibly aggregate upon meeting each other, held together by the attractive van der Waals forces. The force keeping them apart can arise either from electrostatic repulsion between their like charged surfaces or from steric repulsion. The latter occurs when hair-like polymer molecules which 'prefer' to be near the solvent than to each other are attached to the surface. When two such particles approach, a repulsive force manifests to avoid interdigitation and/or compression of the polymers, which would derive them of their precious solvent molecules. The combination of these attractive and repulsive interactions has energy on the order of the thermal energy, meaning that there is still a chance of the particles spontaneously sticking together. How likely this is depends on the details of the attractive and repulsive forces.

The interaction energies being close to the thermal energy at room temperature means that materials composed of colloidal particles are readily deformed by external forces. It is for this property that such materials are known as soft matter, an intuitive name for things like the squidgy modelling clay discussed in the previous section. It remains reasonable for the dried clay before it enters 
the kiln, which behaves like a soft rock akin to chalk ${ }^{2}$. Upon firing however, the atomically bonded links between the particles reduce the thermal motion to tiny vibrations and at this point the material graduates out of the field of soft matter and the issue is handed over to the aptly named solid-state physicist. At the other extreme, when presented only with the dilute dispersion (muddy water), the meaning of 'soft' is less clear and one must revert to the more abstract concepts of softness as being related to the particle interactions and the thermal energy.

\subsection{The Toolkit}

There are myriad ways to study colloidal systems, with the muddy-water light scattering experiment in Section 1.1 being a perfectly valid one. In this section, three of the main tools which are available to the modern colloid scientist are introduced. With these, the colloidal domain may be studied to increase our understanding, as well as leveraged to do our bidding.

\subsubsection{Creation: Colloidal synthesis}

Being able to create colloidal particles custom designed for a purpose provides many opportunities in both fundamental research and applications. For the former, the simplest system is often the most useful, at least to begin with. In colloid science, the base system is one consisting of identical hard spheres, whose interaction is completely isotropic and acts only to prevent overlap when two particles touch.

Real colloids are often born through nucleation and growth from a complex chemical soup which yields particles with a range of sizes, complicating the comparison with theory. The ability to minimise the size dispersity in a system of colloidal particles is therefore an important tool. At present, the most readily synthesised monodisperse systems are particles of organic polymers or silica.

Clear from Section 1.2 is the importance of also controlling the particle surface properties. For charge stabilised particles, alteration of the $\mathrm{pH}$ or salt concentration can change the interactions to make the particles more or less stable. Charged chemical species can also be attached to the surface to change the magnitude and/or sign of the surface charge. In non-polar solvents where the particle surface less readily becomes charged, the attachment of polymer chains for steric stabilisation may be used to control the particle interactions.

${ }^{2}$ Chalk is also a hierarchical material, being formed by sedimentation of colloidal calcite platelets left behind by prehistoric algae [6]. 


\section{Introduction}

\subsubsection{Observation: Microscopy}

The upper limit of the colloidal size range may be debatable, but there is no questioning that it lies well below what our eyes can resolve unassisted. Therefore to see the individual particles and observe their behaviour, some sort of microscope is required. Electron microscopes enable the closest examination of the particles, being able to resolve surface features on the nanoscale. This is an important tool for particle characterisation but the study of many colloidal phenomena is impossible in the vacuum of the electron column ${ }^{3}$.

What the more old-fashioned optical microscope lacks in resolution, it gains in versatility. Here, particles can readily be observed while suspended in liquids and a compound microscope of the type the reader may remember from school is sufficient to observe individual colloids engaged in their mesmerising Brownian dance. Upping the tech-level with fluorescent colloids and a confocal microscope which can image thin cross sections of a thick sample, $3 \mathrm{D}$ volume images (and videos) may be captured.

The confocal method pushes the resolution of the microscope to a fundamental physical limit, which is determined by the diffraction of light. Recently, a range of 'super-resolution' techniques have emerged which sneak around this limit in various ways, to resolve ever finer details [8]. One of the tricks is to somehow separate the illumination of the unresolvable objects in time as well as space. This may be illustrated with the example of taking a photograph of two lightbulbs which are close together and far away from the camera. When photographed together, only a single blob of light is visible but if two pictures are captured in sequence with only one of the lights on at at time, comparison of the two photographs will reveal the separate positions of the bulbs.

\subsubsection{Manipulation: Phoresis}

Ways to manipulate colloidal dispersions may be placed on a spectrum of granularity (or selectivity), with global methods like pouring or pumping the whole dispersion at one end and single particle choreography with optical tweezers at the other. In between, we may place sedimentation under gravity closer to the global end, and phoretic transport between this and the local end. This last is defined as colloidal migration due to a field interacting with the particle surfaces [9]. Examples are electrophoresis in an electrical potential gradient (i.e. an electric field), thermophoresis in a temperature gradient and diffusiophoresis in a gradient of concentration of a chemical species. These gradients induce fluid flows along the particle surface and cause it to move.

\footnotetext{
${ }^{3}$ Liquid phase electron microscopy is an emerging technique with the promise to change this [7].
} 
The reason that gravitational sedimentation is not a phoretic effect is a subtle question of force. The difference may be illustrated with a thought experiment involving a screw immersed in a fluid. The case of sedimentation is clear: a net force is exerted on the particle by the gravitational field causing it to accelerate until the drag force balances the gravitational one. If we then consider the screw to be somehow made to rotate around its long axis, then it will be propelled forwards by pulling fluid along its surface and effectively swapping places with the fluid in front of it. There is no net force on the combined system of screw and swapping-fluid. This lack of a net force causing movement is characteristic of phoretic transport, as is the confinement of the disturbed fluid to a region very close to the particle surface [9].

Dependence on the surface properties is what makes phoretic manipulation more specific than sedimentation. Approaching the usefulness limit of our screw analogy, we could distinguish two screws with different sizes and/or densities by watching them sink in the liquid. On the other hand, our phoretic analogue could not only distinguish size but also screws with different surface properties, i.e. the handedness and pitch of the threads. The relevant surface parameters of colloidal particles are the sign and magnitude of the surface charge as well as the electrolyte concentration. These govern the behaviour of the electric double layer around the particle, and hence that of the fluid involved in phoresis. A feature which is lost in the analogy of the screw is the non monotonous relationship between the surface charge (zeta potential) and the velocity (both magnitude and direction) of phoretic migration.

\subsection{The work}

The pebble to be added to the cairn atop the mountain of scientific achievement by this thesis is a little more knowledge of the cutting edges of the above tools and of how these edges may be honed.

Chapters 2 and 3 deal with the creation of colloidal matter, in the form of solid spherical particles and oil droplets respectively. The solid fluorescent silica colloids were made by a combination of methods, one older and one newer. Seed particles were synthesised using a relatively new amino acid catalysed route and additional layers were grown around these using more well established techniques. The relative sizes of the core, dyed layer and spacer layer were carefully tuned to make a model system suitable for study with super-resolution optical microscopy. With just the right proportions, single particles may be resolved without the spacer layer becoming so large that gravitational sedimentation dominates.

In a different example of colloidal creation, the oil droplets of Chapter 3 


\section{Introduction}

were made using microfluidics. Here, droplets are made serially at a nozzle resembling a microscopic dripping tap. Such methods are well known for their production of uniformly sized droplets, albeit relatively slowly and usually at sizes larger than the colloidal limit. It is these latter two issues which are addressed in Chapter 3. Results are presented from a parallelised dropmaking device which contains thousands of nozzles all creating colloidal droplets of diameter $1 \mu \mathrm{m}$.

Observation by microscopy is represented by Chapter 4. Therein, the resolution limits of a commercial super-resolution (specifically: $\mathrm{STED}^{4}$ ) microscope are probed at different imaging depths using fluorescent silica particles small enough to approximate point light sources. The response to such a source is the point spread function (PSF) which completely characterises the information gathering ability of the microscope. An attempt was also made to use this characterisation to extract more information from microscopic data, by depth dependent deconvolution.

Finally, attention is turned to colloidal manipulation in the form of diffusiophoresis in Chapter 5. This is a specific case of phoretic migration due to a gradient in solute concentration. Combining a salt gradient constructed using a microfluidic device with single particle confocal microscopy, the phoretic transport was measured. With such detailed data, direct comparison with current theories of diffusiophoresis could be made to increase understanding of how colloidal particles may be precisely controlled using imposed salt gradients.

\footnotetext{
${ }^{4}$ Stimulated Emission Depletion, in reference to the method of temporal separation of fluorescent signals, by darkening the adjacent fluorophores using stimulated emission.
} 


\title{
2 Synthesis of monodisperse fluorescent silica nanospheres
}

\begin{abstract}
Synthetic routes to model systems of spherical silica nanoparticles with diameters in the range $30-360 \mathrm{~nm}$ are presented. The general method uses nanoparticles formed by an arginine catalysed method as seeds for growth of Stöber-type silica. In this way, fluorescently dyed and uniformly sized particles could be made with diameter as low as $30 \mathrm{~nm}$ (polydispersity $<7 \%$ ). By growing the particles to diameter $360 \mathrm{~nm}$ (polydispersity $<2 \%$ ), the possibility of imaging single particles in $3 \mathrm{D}$ with super-resolution optical microscopy was opened up, while keeping the gravitational length of the particles more than $50 \times$ greater than the particle diameter. Examining the resulting particles, preliminary results suggest the arginine catalysed growth is surface reaction limited, with polydispersity inversely proportional to the particle diameter. Finally, with a modified particle surface, dispersion in non polar organic solvents was demonstrated, showing the versatility of this model colloidal system.
\end{abstract}




\subsection{Introduction}

The packing behaviour of spheres has been of interest to humanity since at least 1591, when Walter Raleigh ${ }^{1}$ wanted to calculate the number of cannonballs in a stack and his friend Thomas Harriot provided him with a formula [10]. In the intervening time, atomic theory has been proven, lending fundamental relevance to the sphere packing problem and bringing this niche interest of $16^{\text {th }}$ century gunners to the attention of physics and chemistry undergraduates the world over. Wishing to model the behaviour of atoms, cannonballs are rather limited in predictive power since their arrangement is determined by forces which do not behave at all like those acting on atoms. Where atomic behaviour is governed by quantum mechanics and may be described by the laws of thermodynamics, that of cannonballs is determined principally by their initial configuration (e.g. how they were put into the box) and by gravity.

A model system which takes a step closer to an accurate representation of atomic behaviour is that consisting of colloidal particles. These are able to sample different configurations by Brownian rearrangement and eventually reach thermodynamic equilibrium. While colloids do sink (or float) under gravity, their thermal motion allows them to jiggle around enough to find the arrangement that minimises the free energy of the system. Just how much jiggling is possible in a gravitational field may be characterised by the ratio of the thermal energy to the gravitational force on a particle ${ }^{2}$, the 'gravitational length', $l_{g}=k T / m g$ [11]. This length may be thought of as 'how far a particle is likely to be kicked up against gravity by Brownian motion'. Atoms, colloids and cannonballs all have a thermal energy per particle of $\sim k T$. The different (buoyant) masses of these mean that the gravitational length of a cannonball is completely negligible, that of an atom or molecule is large $(\sim 9 \mathrm{~km}$ for $\mathrm{N}_{2}$ ) and that of a silica colloid is somewhere in between. More specifically, colloidal particles can have gravitational lengths on the order of the particle size. Figure 2.1 shows the variation of the gravitational length with diameter for silica colloids in water. As $l_{g}$ depends on the inverse of the cubed diameter, a small increase in diameter drastically reduces $l_{g}$, reaching $100 \times$ the particle diameter at $D \sim 300 \mathrm{~nm}, 10 \times$ at $\sim 500 \mathrm{~nm}$ and $l_{g}=D$ at $\sim 1 \mu \mathrm{m}$. It is not impossible to reach thermodynamic equilibrium with $l_{g}=D$, but $l_{g}=10 D$ is a safer threshold.

It being possible to reach thermodynamic equilibrium is no guarantee of getting there any time soon. The equilibration time depends not on how much

\footnotetext{
${ }^{1}$ Not to be confused with Lord Rayleigh, who would be born some 250 years later.

${ }^{2}$ The thermal energy is given by the Boltzmann constant, $k$, multiplied by the absolute temperature, $T$. The net force on a dispersed particle is the product of the buoyant mass, $m$, and the gravitational acceleration, $g$.
} 


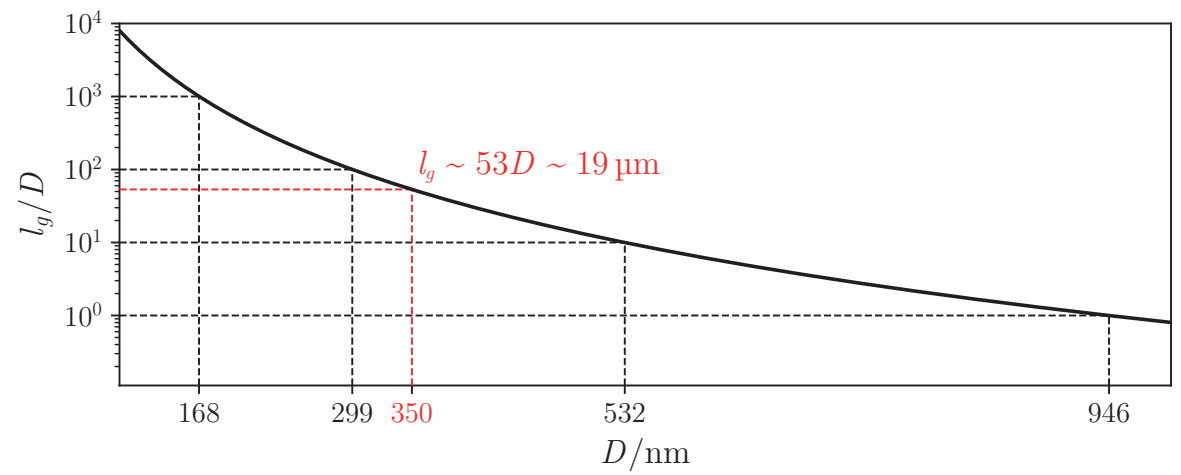

Figure 2.1: Calculated gravitational length, $l_{g}$, of spherical silica colloids in water as a function of diameter, $D$. The black dotted lines indicate the diameters at which the gravitational length reaches various multiples of the particle diameter. The red dotted line indicates the gravitational length of the target $350 \mathrm{~nm}$ diameter particles of this chapter, chosen to maximise $l_{g}$ while maintaining the possibility of single particle confocal microscopy.

the particles are jiggling around, but how quickly they are doing so. The time required for a free particle to diffuse over its own diameter scales with $D^{3}$, so the dynamics are much faster in systems of smaller particles.

Why not push the particle size down as far as possible to maximise $l_{g}$ and minimise equilibration times? This would negate the reason for using colloids rather than atoms themselves: colloids can be large enough and move slowly enough to be imaged with in situ optical microscopy. Therefore there is a lower limit on the useful size of particles set by the spatial and temporal resolution of the microscope to be used to study them.

Apart from being a suitable size for microscopic study, the interactions between colloids may be readily tuned by modification of the particle surfaces and/or properties of the dispersing solvent, making them a versatile model system. Monodisperse colloidal spheres have therefore been extensively studied and, for example, the earlier predicted [12] entropy driven crystallisation of hard spheres was demonstrated with such a system [13].

In reality, systems of colloidal particles always contain some finite size dispersity, which affects the equilibrium phase behaviour. Specifically, increasing polydispersity destabilises the crystal with respect to the fluid phase, as the lattice must accommodate increasingly disparate particle sizes [14, 15]. Hard 


\section{Synthesis of monodisperse fluorescent silica nanospheres}

spheres (HS) have long been observed to be able to crystallise up to a polydispersity $(\delta$, relative standard deviation in the diameter distribution) of $\sim 7 \%$, above which amorphous phases dominate at high density [16-18]. Above this 'terminal polydispersity', formation of the classic face centred cubic (FCC) crystal is no longer possible and the phase behaviour becomes more complex and is dominated by the polydispersity ${ }^{3}$.

Spherical colloids may be made from a number of materials, with silica and organic polymers being the most well established. Both of these facilitate synthesis of (almost) monodisperse spherical particles across a range of sizes within the colloidal range [23]. The reason for the prominent position of silica stems largely from the work of Stöber, Fink and Bohn who in 1968 discovered a way to make monodisperse silica colloids with diameters ranging from around $50 \mathrm{~nm}$ to $2 \mu \mathrm{m}$ by condensation of an alkoxysilane precursor in alcohol/water with an ammonia catalyst [24]. Since then, methods have been found to change the size of the particles by growing shells of silica around them, in the process also reducing the relative width of the size distribution [25, 26]. Furthermore, it is possible to incorporate dyes into the particles, allowing them to be studied with fluorescence microscopy [27].

In 2006, a new route to colloidal silica spheres was discovered by Yokoi et al. [28]. In contrast to the Stöber method, this involves two liquid phases: an oily (tetra)alkoxysilane containing phase and an aqueous solution of a basic amino acid (lysine or arginine). This method yields low polydispersity particles with diameter around $12 \mathrm{~nm}$, considerably below the lower limit accessible using the Stöber process.

Building upon this discovery was the comprehensive work of Hartlen et al. in which the accessible size range was greatly extended using seeded growth [29]. Using an arginine catalyst, growth up to $167 \mathrm{~nm}(\delta=2 \%)$ was demonstrated as well as the incorporation of fluorescent dye inside the particles. By switching to conventional seeded Stöber growth, larger particles could be made. With this detailed study, the method became well established and is often referred to in literature as the "two phase method" . Particles made in this way have since been frequently applied in the assembly of photonic crystals [30-37] and the fluorescently dyed particles have found application in biological mapping [38].

An important distinction between dyed particles is whether the fluorophore

${ }^{3}$ At high polydispersity, crystal phases may still be formed via size segregation [19]. This requires rearrangement in dense suspension which is extremely slow and thus the timescale for such HS crystallisation is well beyond the typical experimental one for $1 \mu \mathrm{m}$ colloids. However, fractionation into exotic binary phases has been demonstrated for systems of $8 \mathrm{~nm}(\delta=14 \%)$ nanoparticles with almost hard interactions [20]. Furthermore, it was recently suggested by computer simulations that a range of binary crystal phases may be formed in systems with polydispersity up to $19 \%$ with very fast dynamics/long equilibration times $[21,22]$. 
loading allows visualisation of (sufficiently well separated) individual particles or only of particle ensembles in a fluorescence microscope. The dyed particles from the amino acid method reported thus far have mostly fallen into the former category. One notable exception was recently presented in Reference 39. Single particle imaging is an important feature if these particles are to find application as a colloidal model system.

In the present study, an almost monodisperse $(\delta<2 \%)$ smooth spherical particle system suitable for single particle imaging in dense suspension was made. As referenced above, this requires a sufficient quantity of fluorescent dye to be incorporated into each particle as well as separation of the fluorescent particles. Separation of the dyed cores may be achieved by adding non fluorescent silica around a dyed core such that even when the particles are almost touching in a dense phase, the fluorescent signals remain resolvable in the microscope. For this work, the somewhat arbitrary target particle diameter of $350 \mathrm{~nm}$ was chosen for having a reasonably high $l_{g}$ of $53 D \sim 19 \mu \mathrm{m}$ and a structure consisting of a $100 \mathrm{~nm}$ dyed core with a $125 \mathrm{~nm}$ thick spacer shell around it to facilitate single particle volume imaging in a $3 \mathrm{D}$ STED microscope.

The various synthetic routes to this goal are summarised in Figure 2.3 in Section 2.4. It was found that addition of dye during two phase growth incorporated insufficient fluorophores for single particle imaging. Therefore a Stöber-type method was used to grow a dense dyed silica layer around the seeds [40], followed by a non fluorescent spacer layer.

This chapter is focussed on the synthetic routes to the desired particles as well as understanding why they are successful (or not) in terms of the particle growth mechanism. First an overview of previous work on the two phase method is presented, along with a more limited discussion of the well established Stöber method to put the latter growth steps into context. The experimentally probed methods are presented, followed by the particles resulting from them along with a discussion of why each of the observed particle systems was formed and how each relates to the goal of this chapter. The possibilities of single particle imaging and surface modification are then demonstrated. Finally, suggestions for future work are given.

\footnotetext{
${ }^{4} \mathrm{~A}$ closely related method of amino acid catalysed seed synthesis and growth modifies this with the addition of ethanol which allows homogenisation of the reaction mixture. This is also frequently applied and allows access to a similar size range as the Hartlen method [38, 41-45].
} 


\subsection{Theory}

\subsubsection{Particle formation and growth in the amino acid catalysed method}

Several efforts have been made to elucidate the phenomena at play in the amino acid catalysed formation of silica particles [43, 46-49]. Perhaps the most convincing of these is presented in Reference 49, in which in situ pH data, transmission electron microscopy ( $\mathrm{TEM}$ ) and ${ }^{29} \mathrm{Si}$ spectra were combined to deduce a mechanism. In the lysine catalysed synthesis, it was observed using cryogenic TEM that the resulting $\varnothing 24 \mathrm{~nm}$ particles were composed of $1.3 \mathrm{~nm}$ primary particles. The mechanism involves initial formation of diffusively charged 'clouds' which densify to form the primary particles. These then associate to form assemblies of around $\varnothing 5 \mathrm{~nm}$ which are the precursors to the main particles. The primary particles are continually formed at around the same size and add to the assemblies in the growth stage of the reaction. This hinges on the idea that the primaries are more stable to each other than to the assemblies such that there is only one short period of association at the beginning of the reaction.

A short initial association period is consistent with destabilisation due to a peak in ionic strength caused by a maximum in the silicic acid concentration. An early conductivity peak is known to occur in the Stöber process before condensation balances hydrolysis [50]. The same almost certainly occurs in the amino acid synthesis, as indirectly evidenced by the early minimum in $\mathrm{pH}$ in Reference 49.

The primary particles are still visible in the main particles in $\mathrm{TEM}$, but with a smaller size of around $1.3 \mathrm{~nm}$, attributed to a further structural collapse upon association. This structural collapse was confirmed by the observation of increasingly condensed silica (higher $\mathrm{Q}^{4} / \mathrm{Q}^{3}$ ratio) from primary to assembly to final particles. This reaction mechanism is consistent with earlier small angle x-ray scattering (S A X S) studies in which it was found that the number density of particles was constant after the initial nucleation event [43, 46, 51].

\subsubsection{Control parameters in the two phase method}

Figure 2.2 shows the various factors involved in the two phase synthesis method, both directly and indirectly controllable. These are discussed below in terms of their names and letter labels in Figure 2.2.

Salt Concentration: Additional salt may be explicitly added to the reaction to (a) increase the ionic strength of the aqueous phase. Catalyst Concentration: The added amino acid is itself an ionic species and so (b) 


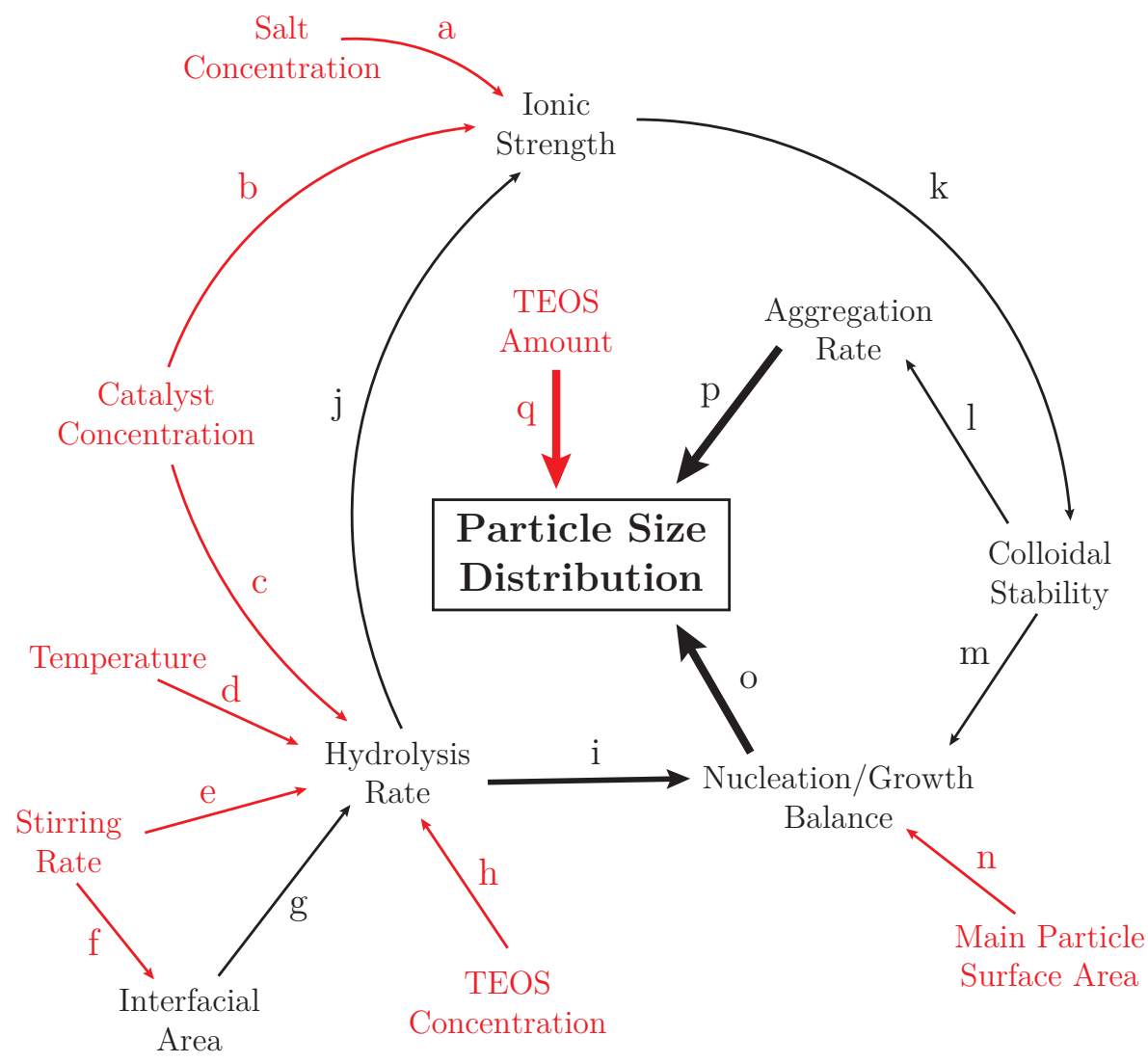

Figure 2.2: Interdependence of factors affecting the resulting particle size distribution in the two phase synthesis. Arrows may be read in the forward direction as this - affects $\rightarrow$ that. Directly controllable experimental parameters are highlighted in red and causal relationships are labelled with letters. Explanations of the terms and the letters may be found in the text of Section 2.2.2.

contributes to increasing the ionic strength. As it is the catalyst, an increased concentration (c) increases the rate of TEOS hydrolysis at the oil-water interface. Temperature: Elevation of the temperature also (d) increases the rate of hydrolysis. Stirring Rate: By transporting the hydrolysis products away from the interface, faster stirring (e) increases the hydrolysis rate. If the stirring is fast enough to disturb the water-oil interface, increasing the stirring 
rate (f) increases the interfacial area of the metastable emulsion. Interfacial Area: A larger interfacial area increases the fraction of TEOS exposed to the aqueous phase and hence (g) increases the rate of hydrolysis. TEos Concentration: The less diluted the TEOS is in the oil phase, the more TEOS is exposed to the aqueous phase thus (h) increasing the rate of hydrolysis. Hydrolysis Rate: Hydrolysis enriches the aqueous phase with silicic acid, increasing saturation and (i) pushing the balance towards nucleation of new particles. This acidic species dissociates in the basic medium and (j) contributes to the ionic strength until it is condensed into silica. Ionic Strength: Ions screen the surface charge which stabilises the silica particles, so at high ionic strength, (k) the stability of the particles is decreased. Colloidal Stability: If the main particles are unstable, (l) they will aggregate. If these are stable but the protoparticles unstable, the protoparticles add to the main particles and $(\mathrm{m})$ the balance is pushed towards growth. If colloidal stability is high, the protoparticles form stable assemblies, pushing the balance towards nucleation. Main Particle Surface Area: With a high surface area of main particles to add to, protoparticles are likely to add to a main particle before forming a stable secondary assembly, thus (n) the balance is pushed towards growth. Nucleation/Growth Balance: This balance determines whether or not seeded growth of the already present particles takes place, or new particles nucleate. Therefore this (o) controls the size of the final main particles and the presence and size of any secondary modes in the distribution. Aggregation Rate: If the main particles aggregate, (p) the particle shapes deviate from spheres and the size and shape monodispersity of the system are disrupted. Teos Amount: The total atom amount of silicon added to the reaction determines the endpoint (q) and the final sizes of the particles are controlled by how much they may grow before the silicon runs out.

\subsubsection{Stöber Silica}

The older Stöber process is in many ways similar to the arginine catalysed one that stemmed from it, also utilising the base hydrolysis and condensation of an alkoxysilane. In terms of the control parameters depicted in Figure 2.2, the only one which no longer applies at all is the interfacial area since TEOS is miscible with ethanol and so the there is no liquid-liquid interface. This more homogeneous system means that the mechanism of particle formation is also rather different from the arginine catalysed method.

Despite the large amount of effort devoted to the topic, consensus has not yet been reached on a general mechanism of Stöber particle formation and growth [52]. While it is generally agreed that the process begins with nucleation of some sort, there is contention about whether the particles grow by 
aggregation of smaller particles/ogliomers, by addition of monomers or by some combination of the two.

For the purposes of this chapter, the view is taken that the general mechanism is a combined one with aggregation dominating at the beginning of reaction, monomer addition dominating at the end and the experimental conditions determining the balance between these. This approach has been shown often to explain experimental results [25,53-55], and it will be seen that it is consistent with the results in this chapter.

\subsubsection{Polydispersity in seeded growth}

The differently sized particles comprising a polydisperse system may compete for monomers with different efficiencies, resulting in different growth rates and changes to the final relative width of the particle size distribution. The rate (and sign) of polydispersity change during seeded growth is of interest for both applied and fundamental reasons. When striving for a monodisperse particle system, the polydispersity must decrease upon particle growth and how quickly it does so determines how much a set of seed particles will need to be grown to reduce the polydispersity below the required threshold. As the rate of polydispersity change (with respect to diameter) depends on competition in time for monomers, kinetic insights may be gained by following the polydispersity evolution during seeded growth. For Stöber type growth, the dependence of polydispersity on particle size has been extensively studied in the context of the growth mechanism $[25,56,57]$. This route has not yet been investigated for the amino acid catalysed method and it would seem that the various particle systems studied in this chapter provide a suitable dataset to elucidate the relationship, and hence the dependence of the reaction rate on particle size.

The changing volume of a growing particle obeys a rate equation of the form $[25,56]$ :

$$
\frac{d V_{p}}{d t} \sim k_{c} c_{m} R^{\alpha}
$$

$V_{p}$ is the particle volume, $k_{c}$ a rate constant, $c_{m}$ the monomer concentration ('monomers' here referring to the units adding to the particles, so likely primary particles in amino acid case) and $R$ the particle radius. The exponent $\alpha$ characterises the power law dependence of the growth rate on the particle size and therefore depends on the growth mechanism ${ }^{5}$. If the growth rate is determined by the attachment rate of units to the surface (surface reaction limited), the rate depends on the particle surface area. Therefore for a sphere which is

${ }^{5}$ This definition of $\alpha$ is consistent with Reference 25 , but is $3 \times$ greater than $\alpha$ in References 56 and 57, where the rate equation is in terms of $V_{p}$ rather than $R$. 
smooth on the scale of the monomer, $\alpha=2$. For a completely penetrable sphere in which every comprising monomer is an available attachment site, $\alpha=3$. For a rough surface, $\alpha$ corresponds to the surface fractal dimension and takes values between 2 and $3[56,58]$. For a polydisperse system, the difference in growth rate of the differently sized particles depends on the value of $\alpha$.

This difference in growth rate determines the polydispersity, $\delta$, of the grown particles and it may be shown that

$$
\delta \propto\langle D\rangle^{\gamma}
$$

where $\langle D\rangle$ is the mean diameter of the distribution and $\gamma=\alpha-3$ for $(\alpha>$ $\left.\frac{3}{2}\right)$ [57]. For Stöber type growth, values of $\gamma$ close to $-1(\alpha=2)$ were measured, thus confirming reaction limited growth [25, 56, 57].

An alternative route to determine $\alpha$ and hence probe the kinetics involves following the growth of a bidisperse system [25, 59]. Instead of following changes in polydispersity to find out how differently sized particles compete for monomers, the mean diameters of the two modes in the size distribution are measured. This is much easier to determine accurately.

It may be shown for growth of a bidisperse system that

$$
D_{s}^{3-\alpha}-D_{0 s}^{3-\alpha}=D_{b}^{3-\alpha}-D_{0 b}^{3-\alpha},
$$

where $D_{0 s}$ and $D_{0 b}$ are the diameters of the small and big seed particles respectively [59]. These seeds are grown to larger diameters $D_{s}$ and $D_{b}$. Solving for the diameter ratio yields

$$
\frac{D_{b}}{D_{s}}=\frac{D_{0 s}}{D_{s}}\left[\left(\frac{D_{s}}{D_{0 s}}\right)^{3-\alpha}+\left(\frac{D_{0 b}}{D_{b}}\right)^{3-\alpha}-1\right]^{\frac{1}{3-\alpha}}
$$

With measurements of the mean diameters of the two modes in a bidisperse system before and after competitive seeded growth, Equation 2.4 may be used to determine $\alpha$.

\subsection{Methods}

\subsubsection{Materials}

Tetraethyl orthosilicate ( TE O s, 98\%), (3-aminopropyl)triethoxysilane (A P S, $\geq$ $98 \%$ ), octadecyl trimethoxysilane ( o T M Os, technical grade, 90\%), L-arginine (reagent grade, $\geq 98 \%$ ), butylamine $(99.5 \%$ ), ammonium hydroxide solution ('ammonia', $28 \mathrm{w} \% \mathrm{NH}_{3}$ in $\mathrm{H}_{2} \mathrm{O}$ ), rhodamine B isothiocyanate ( $\mathrm{R}$ I T C, mixed 
isomers), fluorescein isothiocyanate (FITC, isomer I, $\geq 90 \%$ ), cyclohexane (reagent grade, $\geq 99 \%$ ) and toluene (technical grade) were obtained from Sigma-Aldrich and not purified further. Deionised water was obtained from a Merck Milli-Q system $(\rho=18.2 \mathrm{M} \Omega \mathrm{cm})$.

All syntheses were carried out in round bottomed flasks quantitatively cleaned with potassium hydroxide. Temperature control was achieved using metal mantles (IKA Flask Carrier). With these mantles, the reaction temperature is usually lower than that of the mantle (e.g. for a mantle temperature of $70{ }^{\circ} \mathrm{C}$, the reaction temperature was around $60^{\circ} \mathrm{C}$ ) so the liquid temperature was also measured and that is reported in method descriptions.

$40 \mathrm{kHz}$ sonication was carried out in a Branson CPX2800 ultrasonic bath operating at full power.

\subsubsection{Notation}

Much of this chapter concerns particles which were grown first with the arginine catalysed method and then by the Stöber process. With many systems having similar particle sizes, additional specification is required to avoid confusion. Therefore the notation ${ }_{\delta}^{c} D$ is used, in which $c$ is the diameter of the 'core' grown with the arginine method, $D$ is the mean full particle diameter and $\delta$ is the diameter polydispersity as a percentage.

For example, ${ }_{5}^{20} 40 \mathrm{~nm}$ denotes a system of particles which were grown to $20 \mathrm{~nm}$ with the arginine method before Stöber silica was grown to make the final particle diameter $40 \mathrm{~nm}$ and the final polydispersity $5 \%$. When $c=D$, the particles were made with the amino acid method without addition of a Stöber shell.

\subsubsection{Aqueous Seed Synthesis}

Fluorescent silica nanoparticles were prepared according to the method described in Reference $38.174 \mathrm{~mL}$ of deionised water containing dissolved arginine $(276 \mathrm{mg}, 9.10 \mathrm{~mm})$ and RITC $(30.1 \mathrm{mg}, 0.32 \mathrm{~mm})$ was heated to $60{ }^{\circ} \mathrm{C}$ in a $500 \mathrm{~mL}$ flask. While vigorously stirring $(600 \mathrm{rpm}), 11.3 \mathrm{~mL}$ TEOs $(50.6 \mathrm{mmol})$ was pipetted under the surface of the hot solution. The approach of reaction completion was indicated by the disappearance of the oily TEOS layer. To ensure complete reaction, the mixture was not cooled and stored until at least $36 \mathrm{~h}$ after TEOS addition.

To prepare particles with differently coloured fluorescence, the procedure described above was repeated but with FITC $(0.30 \mathrm{~mm})$ in place of RITC. In both cases, only a portion of the dye was incorporated in the particles (as indicated by a fluorescent supernatant after centrifuging all particles to the 
bottom). Finally, the same method with no dye added was used to obtain non-fluorescent silica nanoparticles. The resulting particles of all three types are shown in Figure 2.4 (Section 2.4.1).

\subsubsection{Direct growth}

'Direct growth' here means increasing the particle size by adding further TEOS to the completed seed synthesis reaction. The seed reaction described above was scaled down by a factor of 5.3 to suit a $100 \mathrm{~mL}$ flask. $24 \mathrm{~h}$ after the first TEOS addition, a further $2.265 \mathrm{~mL}(10.1 \mathrm{mmol})$ of TEOS was added with stirring kept at $600 \mathrm{rpm}$ and temperature at $60^{\circ} \mathrm{C}$. This addition was repeated every $24 \mathrm{~h}$ for a total of 6 growth steps. Thereafter, $5.00 \mathrm{~mL}(22.4 \mathrm{mmol})$ of TEOS was added every $48 \mathrm{~h}$ for a further 3 growth steps. Before each TEOS addition, a sample was removed and diluted with ethanol and dropcast onto a TEM grid. Images of these samples are shown in Figure 2.5 (Section 2.4.2).

\subsubsection{Diluted arginine growth}

To take larger growth steps and reach larger particle diameters, the seed particles were diluted by around $10 \times$ and the reagent concentrations maintained. The method is similar to that presented in Reference 29. The values were chosen such that the total reaction mixture filled a $500 \mathrm{~mL}$ round bottomed flask to the level of the top of the heating mantle $(\sim 215 \mathrm{~mL})$.

$21.5 \mathrm{~mL}$ of the completed seed synthesis mixture (Section 2.3.3) was added to a $500 \mathrm{~mL}$ round bottomed flask containing $160 \mathrm{~g}$ of arginine solution. The arginine concentration (including arginine added with the seeds) was $9.22 \mathrm{M}$ and the reaction temperature was $60^{\circ} \mathrm{C}$. With stirring at $200 \mathrm{rpm}, 21.5 \mathrm{~mL}$ TEOS was added slowly by pipette to float on top of the aqueous dispersion. The low stirring speed was such that the oil layer had a small central depression, and was unbroken. These conditions were maintained for at least $48 \mathrm{~h}$ before the reaction was allowed to cool to room temperature. The particles were then stored without washing or used as seeds for further growth. In case of further growth steps, the procedure was repeated, each time with $21.5 \mathrm{~mL}$ of seed dispersion and $21.5 \mathrm{~mL}$ of TEOS. The specific concentrations during each growth step are given in Table 2.1 and the resulting particles are shown in Figures 2.6 and 2.7 (Section 2.4.3).

When it was required to reduce the appearance of secondary particles, the conditions were modified. The ionic strength was increased by addition of $\mathrm{LiCl}$, the catalyst concentration was reduced by adding less arginine and the available seed surface area was increased by increasing the concentration of 
silica particles at the beginning of growth. The specific conditions tested may be found in the lower section of Table 2.1.

\begin{tabular}{|c|c|c|c|c|c|c|c|}
\hline $\begin{array}{c}\mathrm{D} \\
(\mathrm{nm})\end{array}$ & $\begin{array}{c}{[\text { Arg. }]} \\
(\mathrm{mm})\end{array}$ & $\begin{array}{c}{[\mathrm{LiCl}]} \\
(\mathrm{mM})\end{array}$ & $\begin{array}{c}{[\text { F I T }]} \\
(\mathrm{mm})\end{array}$ & $\begin{array}{c}{[\mathrm{Seeds}]} \\
(\mathrm{mg} / \mathrm{mL})\end{array}$ & $\begin{array}{c}{[\text { TEOS }]} \\
(\mathrm{M})\end{array}$ & $\begin{array}{c}\text { Volume } \\
(\mathrm{mL})\end{array}$ & Figure \\
\hline $\begin{array}{c}15 \\
12\end{array}$ & 8.5 & 0.77 & 0.28 & 0 & 0.27 & 37 & 2.3 \\
\hline $\begin{array}{c}35 \\
5\end{array} 5$ & 9.2 & 0.84 & 0.31 & 1.6 & 0.44 & 219 & 2.3 \\
\hline${ }_{3}^{83} 83$ & 9.3 & 0.77 & 0.12 & 2.8 & 0.44 & 219 & 2.3 \\
\hline \hline${ }_{3}^{182} 182 /{ }_{6}^{83} 83$ & 9.30 & 0.84 & - & 2.9 & 0.44 & 219 & $2.7 \mathrm{a}$ \\
\hline${ }_{3}^{182} 182 /{ }_{7}^{76} 76$ & 9.30 & 2.3 & - & 2.9 & 0.44 & 219 & $2.7 \mathrm{~b}$ \\
\hline${ }_{3}^{177} 177 /{ }_{10}^{75} 75$ & 9.29 & 23 & - & 2.9 & 0.44 & 219 & $2.7 \mathrm{c}$ \\
\hline${ }_{2}^{166} 166 /{ }_{20}^{51} 51$ & 2.00 & 78 & - & 2.9 & 0.44 & 220 & $2.7 \mathrm{~d}$ \\
\hline${ }_{4}^{156} 156 /{ }_{4}^{58} 58$ & 8.95 & 0.11 & - & 4.3 & 0.66 & 146 & $2.7 \mathrm{e}$ \\
\hline${ }_{4}^{166} 166 /{ }_{10}^{54} 54$ & 2.07 & 0.86 & - & 3.0 & 0.45 & 213 & $2.7 \mathrm{f}$ \\
\hline
\end{tabular}

Table 2.1: Concentrations of reagents in successive arginine catalysed (aqueous) seeded growths. Bidisperse particle systems are indicated by big / small. All systems below the break in the table used the same $83 \mathrm{~nm}$ particles as seeds.

\subsubsection{Stöber growth}

Stöber growth was carried out starting from various particle sizes. The procedures are described in this section in order of increasing seed size.

\section{From $16 \mathrm{~nm}$ seeds}

$155 \mathrm{~g}$ ethanol (absolute) and $2.59 \mathrm{~g}$ deionised water were added to a $500 \mathrm{~mL}$ round bottomed flask and heated to $40^{\circ} \mathrm{C}$ with stirring. $1.22 \mathrm{~mL}$ ammonia $(26.99 \mathrm{w} \%)$ was added before the addition of $15.0 \mathrm{~mL}$ of $16 \mathrm{~nm}$ RITC dyed seeds prepared as described in Section 2.3.3. $2.90 \mathrm{~mL}$ of TEOS was added under the liquid surface by pipette with $600 \mathrm{rpm}$ stirring. The stirring rate was reduced to $200 \mathrm{rpm}$ about $10 \mathrm{~s}$ later and the reaction allowed to proceed with stirring and temperature maintenance for at least $12 \mathrm{~h}$. Particles resulting from this synthesis are shown in Figure 2.8 b (Section 2.4.4).

For further growth, the stirring and temperature were maintained and an additional $6.00 \mathrm{~mL}$ of TEOS was added dropwise with a syringe pump at $0.30 \mathrm{~mL} \mathrm{~h}^{-1}$. This was also allowed to react for $24 \mathrm{~h}$ after the final TEOS addition and the resulting particles are shown in Figure 2.9 a. For the following 


\begin{tabular}{|c|c|c|c|c|c|c|c|}
\hline $\begin{array}{c}D \\
(\mathrm{~nm})\end{array}$ & $\begin{array}{c}{[\mathrm{EtOH}]} \\
(\mathrm{M})\end{array}$ & $\begin{array}{c}{\left[\mathrm{NH}_{4} \mathrm{OH}\right]} \\
(\mathrm{M})\end{array}$ & $\begin{array}{c}{\left[\mathrm{H}_{2} \mathrm{O}\right]} \\
(\mathrm{M})\end{array}$ & $\begin{array}{c}\text { [Seeds] } \\
(\mathrm{mg} / \mathrm{mL})\end{array}$ & $\begin{array}{c}\text { TEOS] } \\
(\mathrm{M})\end{array}$ & $\begin{array}{c}\text { Volume } \\
(\mathrm{mL})\end{array}$ & Notes \\
\hline${ }_{12}^{15} 15$ & - & $\begin{array}{ll}- & \text { - }\end{array}$ & $\begin{array}{ll}- & \text { - }\end{array}$ & - & - & - & Seeds \\
\hline${ }_{7}^{15} 27$ & 15.4 & 0.078 & 0.424 & 1.13 & 0.060 & 218 & \\
\hline${ }_{4}^{15} 82$ & 12.8 & 0.183 & 2.355 & 1.78 & $0.195^{*}$ & 229 & \\
\hline
\end{tabular}

Table 2.2: Reagent concentrations in successive growths starting from $15 \mathrm{~nm}$ seed particles (assuming additive volumes). The ${ }_{5}^{15} 39 \mathrm{~nm}$ intermediate step is omitted since this growth was done by addition of TEOS to the mixture used to grow the particles to ${ }_{7}^{15} 27 \mathrm{~nm}$. The resulting particles are shown in Figures $2.8 \mathrm{~b}$ and 2.9. * Concentration of TEOS is quoted as if it were added all at once when in fact it was added gradually by syringe pump as described in the text.

growth step (result in Figure $2.9 \mathrm{~b}$ ), the synthesis was carried out with $135 \mathrm{~g}$ absolute ethanol, $15.0 \mathrm{~g}$ deionised water, $3.00 \mathrm{~mL}$ ammonia, $30.0 \mathrm{~mL}$ of the previous particle dispersion and $10.00 \mathrm{~mL}$ TEOS added at $0.20 \mathrm{~mL} \mathrm{~h}^{-1}$.

\section{From 33 nm seeds (dyed)}

$126 \mathrm{~g}$ absolute ethanol and $1.50 \mathrm{~mL}$ ammonia were mixed in a $500 \mathrm{~mL}$ round bottomed flask and the temperature raised to $30^{\circ} \mathrm{C} .21 .5 \mathrm{~mL} 33 \mathrm{~nm}$ seed particles in arginine growth mixture $(1.90 \mathrm{mg} / \mathrm{mL})$ were added, followed by $2.00 \mathrm{~mL}$ TEOS.

Around these particles a dyed silica shell was grown using the silane coupling agent A P S as described in Reference 27. First, the dye was coupled to the silane as follows. $90 \mathrm{mg}$ of RITC $(0.168 \mathrm{mmol})$ was dissolved in $10.0 \mathrm{~mL}$ absolute ethanol by shaking and light sonication. $94 \mu \mathrm{L}$ A P S $(0.402 \mathrm{mmol})$ was added to this mixture which was then left on a flat orbital shaker for $24 \mathrm{~h}$. This mixture was then diluted by adding it to $20.0 \mathrm{~mL}$ of absolute ethanol.

For the growth, $30.0 \mathrm{~g}$ of absolute ethanol, $100.0 \mathrm{~mL}$ of the reaction product from the previous step and $0.50 \mathrm{~mL}$ ammonia were mixed in a $500 \mathrm{~mL}$ round bottomed flask. $10.00 \mathrm{~mL}$ TEOS was diluted with $20.0 \mathrm{~mL}$ absolute ethanol and loaded into a syringe. Both the diluted TEOS and APS/dye mixtures were added at $0.70 \mathrm{~mL} \mathrm{~h}^{-1}$ to a total volume of $29.0 \mathrm{~mL}$, corresponding to A P S and TEOS addition rates of 2.2 and $230 \mu \mathrm{L} \mathrm{h}^{-1}$ respectively. After $13 \mathrm{~mL}$ of addition, $5.0 \mathrm{~mL}$ deionised water was added. The reaction was left heated and stirring for $24 \mathrm{~h}$ after the final addition before $0.500 \mathrm{~mL}$ of $\mathrm{TEOS}$ dissolved in $1.0 \mathrm{~mL}$ ethanol was added by pipette. This step was included to add a small additional layer of silica with a reduced concentration of destabilising surface 


\begin{tabular}{|c|c|c|c|c|c|c|c|}
\hline $\begin{array}{c}D \\
(\mathrm{~nm})\end{array}$ & $\begin{array}{c}{[\mathrm{EtOH}]} \\
(\mathrm{M})\end{array}$ & $\begin{array}{c}{\left[\mathrm{NH}_{4} \mathrm{OH}\right]} \\
(\mathrm{M})\end{array}$ & $\begin{array}{c}{\left[\mathrm{H}_{2} \mathrm{O}\right]} \\
(\mathrm{M})\end{array}$ & $\begin{array}{c}{[\text { Seeds }]} \\
(\mathrm{mg} / \mathrm{mL})\end{array}$ & $\begin{array}{c}{[\mathrm{TEOS}]} \\
(\mathrm{M})\end{array}$ & $\begin{array}{c}\text { Volume } \\
(\mathrm{mL})\end{array}$ & Notes \\
\hline${ }_{8}^{33} 33$ & - & - & - & - & - & - & Seeds \\
\hline${ }_{5}^{33} 41$ & 14.8 & 0.12 & 3.6 & 3.4 & 0.05 & 185 & \\
\hline${ }_{4}^{33} 77$ & 9.80 & 0.08 & 2.4 & 2.8 & $0.20^{*}$ & 227 & + Dye \\
\hline${ }_{2}^{33} 200$ & 22.1 & 0.58 & 3.6 & 1.5 & $0.41^{*}$ & 92 & \\
\hline${ }_{2}^{33} 359$ & 13.5 & 0.67 & 4.1 & 3.8 & $0.28^{*}$ & 89 & \\
\hline
\end{tabular}

Table 2.3: Reagent concentrations used to grow the dyed particles (assuming additive volumes). These particles correspond to those shown in Figure 2.10. * Concentration of TEOS is quoted as if it were added all at once when in fact it was diluted 1:2 in ethanol and the mixture added at $0.70 \mathrm{~mL} \mathrm{~h}^{-1}$ using a syringe pump. Additional ethanol added in this way was not included when computing concentrations until the subsequent step.

amine groups compared to that after the dying step. The resulting particles are shown in Figure 2.10 c (Section 2.4.5).

The dyed particles were then washed by centrifuging at $900 \mathrm{~g}$ for $6 \mathrm{~h}$ and replacing the supernatant with ethanol 4 times. The final two growth steps to add a spacer of undyed silica to the particles were carried out in a similar fashion, although in $250 \mathrm{~mL}$ flasks. To avoid repetition, the relevant details are relegated to Table 2.3 .

\section{From larger seeds}

When the switch to Stöber growth was made later, similar methods were used for the growth as in the previous section. The specific concentrations used may be found in Table 2.4. In all cases, the TEOS was diluted 1:2 in ethanol and the mixture added at $0.7 \mathrm{~mL} \mathrm{~h}^{-1}$ using a syringe pump $[26,60]$. In contrast to the early Stöber method, a dyed Stöber shell was not added since F ITC dye was already incorporated during the arginine catalysed seeded growth as described in Section 2.3.5.

In the case of the $165 \mathrm{~nm}$ seed particles, it was necessary to remove the secondary particles which were created during the final arginine growth before beginning Stöber growth. This was done by repeated $(6 \times)$ centrifugation $(900 \mathrm{~g}, 2 \mathrm{~h})$ and redispersion of the $165 \mathrm{~nm}$ particles. The majority of the turbid supernatant was discarded after each centrifugation step and the volume recovered by addition of ethanol. 


\begin{tabular}{|c|c|c|c|c|c|c|l|}
\hline$D$ & {$[\mathrm{EtOH}]$} \\
$(\mathrm{nm})$ & $(\mathrm{M})$ & $\begin{array}{c}{\left[\mathrm{NH}_{4} \mathrm{OH}\right]} \\
(\mathrm{M})\end{array}$ & $\begin{array}{c}{\left[\mathrm{H}_{2} \mathrm{O}\right]} \\
(\mathrm{M})\end{array}$ & $\begin{array}{c}{[\mathrm{Seeds}]} \\
(\mathrm{mg} / \mathrm{mL})\end{array}$ & $\begin{array}{c}{[\mathrm{TEO}]^{*}} \\
(\mathrm{M})\end{array}$ & $\begin{array}{c}\text { Volume } \\
(\mathrm{mL})\end{array}$ & Notes \\
\hline${ }_{3}^{83} 83$ & - & - & - & - & - & - & Seeds \\
${ }_{2}^{83} 201$ & 13.0 & 0.56 & 3.0 & 1.7 & 0.48 & 94 & \\
${ }_{1}^{83} 350$ & 12.2 & 0.62 & 3.8 & 4.7 & 0.24 & 87 & \\
\hline${ }_{1}^{165} 165$ & - & - & - & - & - & - & Seeds \\
${ }_{2}^{165} 330$ & 11.6 & 0.61 & 3.7 & 3.5 & 0.28 & 86 & \\
\hline
\end{tabular}

Table 2.4: Reagent concentrations used for Stöber growth of 83 and $165 \mathrm{~nm}$ seeds (assuming additive volumes). The resulting particles are shown in Figure 2.12. * Concentration of TEOS is quoted as if it were added all at once when in fact it was diluted and gradually added as described in the text.

\subsubsection{Surface modification}

To disperse the particles in non-polar organic solvents, $\mathrm{C}_{18}$ chains were attached to the particles using an unstable to stable method with the silane coupling agent otmos [61]. This was done with two particle sizes: $39 \mathrm{~nm}$ and $330 \mathrm{~nm}$. Before coating, the particles were washed into ethanol to remove remaining reactants, catalyst etc. This was more difficult for the smaller particles due to their lower stability.

To prevent aggregation, the $39 \mathrm{~nm}$ particles required long and gentle centrifugation runs. Specifically, they were centrifuged at $900 \mathrm{~g}$ for $15 \mathrm{~h}$ in $40 \mathrm{~mL}$ glass vials and the supernatant removed and replaced with ethanol. This was repeated at least 3 times. The larger particles could be centrifuged at the same speed for only 15 minutes and then washed in the same way.

For the coating, the silica concentration was adjusted such that the total particle surface area was $\sim 2.8 \mathrm{~m}^{2}$ (e.g. $36 \mathrm{mg}$ of $39 \mathrm{~nm}$ particles). They were then centrifuged once again with the same conditions and the supernatant removed as much as possible without disturbing the sediment. The ethanol level was reduced further by encouraging it to evaporate by flowing nitrogen into the open vial. A mixture containing $8.00 \mathrm{~mL}$ toluene, $0.80 \mathrm{~mL}$ butylamine catalyst and $0.80 \mathrm{~mL}$ OTMOS was added and the vial shaken to partially disperse the unstable colloids. This was then treated with ultrasound in a bath at $40{ }^{\circ} \mathrm{C}$ for about $16 \mathrm{~h}$. The coated particles were then washed using the same speeds as before but this time into toluene for the first wash and into cyclohexane for 3 thereafter. 


\subsubsection{Particle characterisation}

After each growth step a small portion $(\sim 0.5 \mathrm{~mL})$ of the reaction mixture was diluted in $\sim 10 \mathrm{~mL}$ ethanol and this mixture was dropcast onto a butvar coated and carbon sputtered copper grid. After drying, the particles were imaged using an FEI Tecnai 10, 12 or 20 transmission electron microscope (TEM). Scanning electron microscopy (SEM) was done using a Phillips XL 30 FEG microscope. Size distributions were measured by drawing lines across at least 100 particle diameters in digital images (using software iт E M or ImageJ).

\subsubsection{Optical Microscopy}

A $10 \mu \mathrm{m}$ thick film of ${ }_{2}^{33} 359 \mathrm{~nm}$ particles was made by vertical deposition [62]. A \#1.5 borosilicate cover glass was placed vertically in a $20 \mathrm{~mL}$ scintillation vial containing $7 \mathrm{~mL}$ of silica dispersion in ethanol with volume fraction $1.8 \%$. The open vial was then covered with a $400 \mathrm{~mL}$ beaker and the dispersion dried at $50{ }^{\circ} \mathrm{C}$ over a period of 16 hours.

The glass slide with deposited particles was then incorporated into a cell of the type described in Section 4.3.1. The refractive index of the particles was approximately determined by eye ${ }^{6}$ to match that of a $12.7 \mathrm{wt} . \%$ water/glycerol mixture, corresponding to a refractive index of 1.454 [63]. The cell was filled with such a mixture to produce an optically homogeneous sample for volume imaging.

Image volumes were captured using a Leica TCS SP 8 STED 3 X inverted confocal microscope operated in $100 \%$ 3 $\mathrm{D}$ STED mode. The objective was a Leica HC PL APO $93 \times / 1.30$ glyc STED white lens with an 85 w\% glycerol/water mixture $\left(n_{D}^{21}=1.4506\right)$ as immersion. For excitation, a $543 \mathrm{~nm}$ laser line $(80 \mathrm{MHz}$ pulsed white light laser, NKT Photonics) was used and a $660 \mathrm{~nm}$ continuous wave laser provided the depletion light. Fluorescence emission was detected in the range $553-650 \mathrm{~nm}$ by a hybrid detector.

The voxel dimensions in Figure 2.11 are $23 \times 23 \times 80 \mathrm{~nm}$, while the image volume has dimensions $11.6 \times 11.6 \times 14.1 \mu \mathrm{m}$. Deconvolution was done using Huygens software and a theoretical point spread function.

The particle coordinates were fitted in $3 \mathrm{D}$ using Trackpy $[64,65]$. The particle crystalline environments were determined using common neighbour analysis as implemented in OVITO software [66, 67].

${ }^{6}$ By eye here meaning adding glycerol to a concentrated dispersion of particles in water and judging the point of maximum dispersion transparency by looking through the vial after each addition. 


\subsection{Results and Discussion}

The main results of this chapter are the different particle systems which were synthesised. These are summarised in Figure 2.3 and the details of the different results are discussed in the indicated sections.

\subsubsection{Seed Synthesis}

The arginine catalysed seed synthesis was found to be both highly reproducible and robust to changes in conditions. In 14 repeated runs under the same conditions $^{7}$, the mean of the mean diameters was $15.5 \mathrm{~nm}$ with a standard deviation from the mean of the means of $0.95 \mathrm{~nm}$. With changing conditions, the minimum and maximum mean diameters observed were $12 \mathrm{~nm}$ and $17 \mathrm{~nm}$ respectively. The conditions which affected the resulting particle size are discussed in this section.

Figure 2.4 shows TEM images of particles with and without different fluorescent dyes incorporated. The particles in each of the three systems have similar shapes, all being slightly deformed from perfect spheres. Related to this is the size difference between the particle systems. The addition of the fluorescent dyes resulted in increased particle diameter and it appears that the larger the particles, the more spherical they become. This shape trend may be explained by the addition of protoparticles of fixed size. As the relative size of the protoparticles to the main particles decreases, the more homogeneously they may be distributed. The reason for the dyes increasing the particle size is due to the increase in the ionic strength of the reaction mixture, rather than special properties of the dye molecules. This was confirmed by the observance of the same trend after adding lithium chloride salt to the arginine solution instead of fluorescent dye. Increased ionic strength reduces particle stability, meaning that colloidal stability of the primary assemblies is reached at a larger size, yielding a smaller number of larger particles.

More rapid stirring of the reaction vessel decreased the size of the resulting nanoparticles. The stirring rate was always high enough that the TEOs did not float atop the aqueous phase as an oily layer but was a dynamic and unstable emulsion. As indicated in Figure 2.2, this means that faster stirring not only improved the mixing but also increased the interfacial area available for hydrolysis to take place. Thus with faster stirring, the rate of TEOS hydrolysis increased, increasing the dissolved silicate concentration at the time

\footnotetext{
${ }^{7}$ The conditions are those reported in for RIT C dyed particles in Section 2.3.3, scaled down by a factor of 5.3 to fit in a $100 \mathrm{~mL}$ round bottomed flask. The reason for this repetition was a mistaken belief that seeded growth would not be possible with aged seeds. This issue is discussed further in Section 2.4.4.
} 


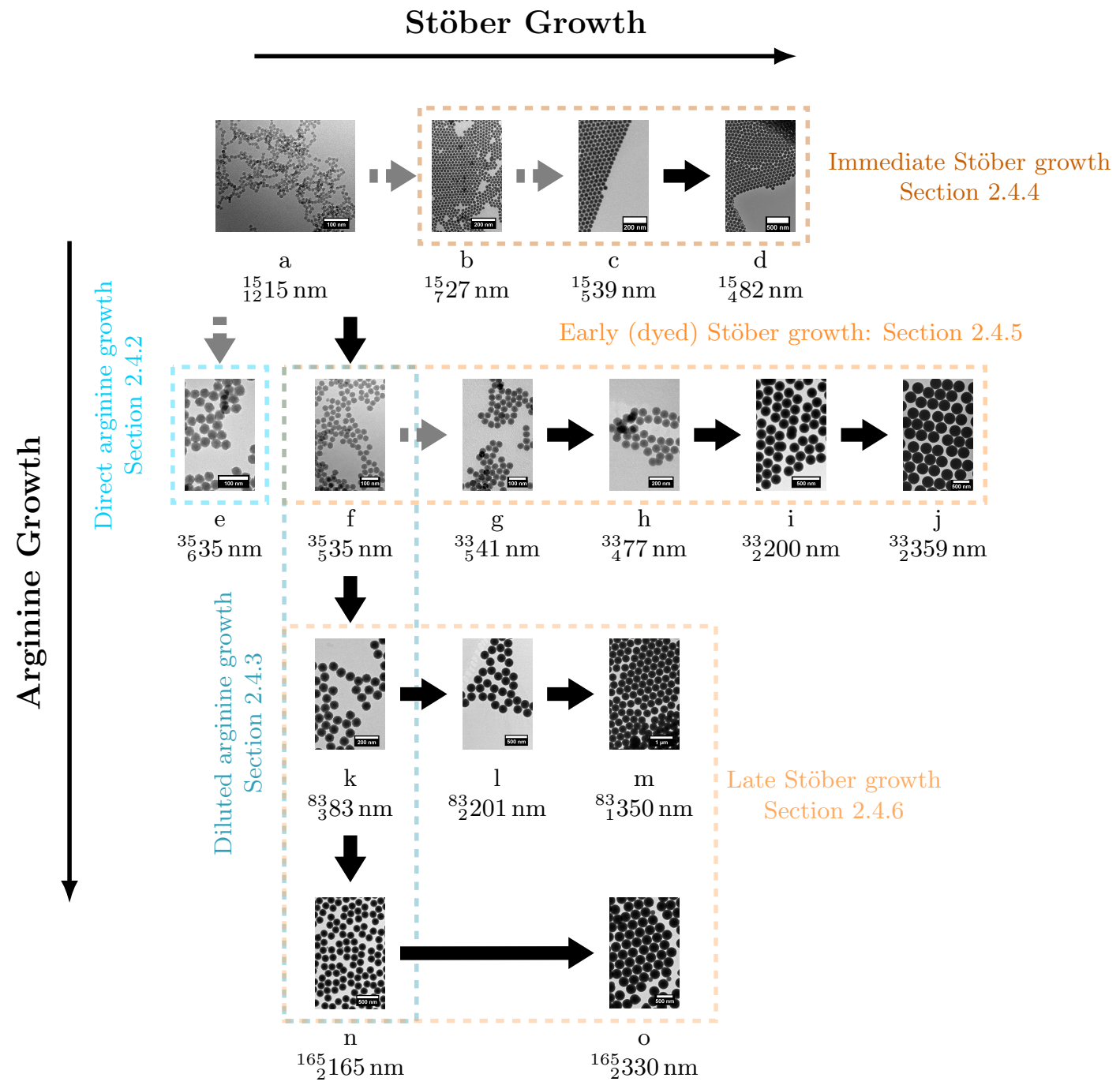

Figure 2.3: Summary of seeded growth protocols in this chapter. The particle sizes are indicated in the compact notation: ${ }_{\delta}^{c} D$, with $c$ the diameter of the core grown with the arginine catalysed method, $D$ the mean total particle diameter and $\delta$ the relative polydispersity in $D$ as a percentage. Solid arrows connect pairs which are successive growths of the same particles while broken arrows indicate that the particles were grown using seed particles comparable to those shown, but not from the same batch. 

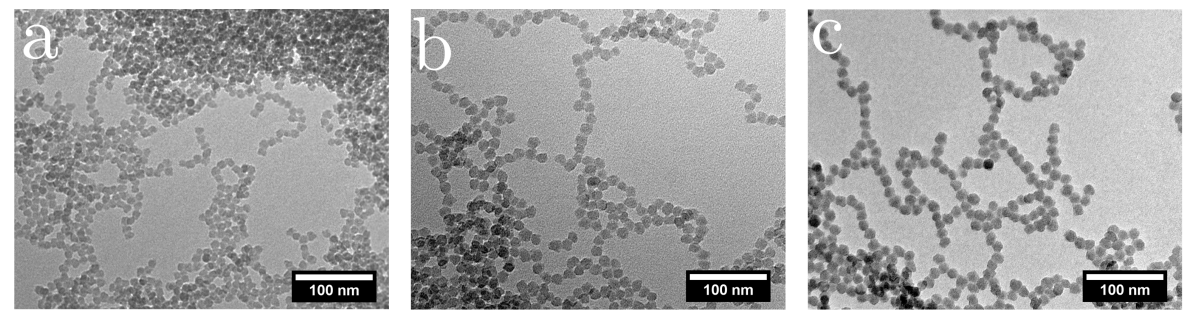

Figure 2.4: Primary particles synthesised via the amino acid catalysed route. a) ${ }_{11}^{12} 12 \mathrm{~nm}$ : without fluorescent dye. b) ${ }_{12}^{15} 15 \mathrm{~nm}$ : with FITC. c) ${ }_{9}^{14} 14 \mathrm{~nm}$ : with RITC.

of nucleation as well as the height of the silicic acid conductivity peak, resulting in a larger number of particles. Each of these grew approximately equally with the end result being the limited silica precursor being divided into a larger number of smaller parts.

The particle size was also observed to decrease upon scaling the reaction volume up. This is likely due to the non-obvious way in which the mixing of the reaction was changed with the larger reaction flask, stirring bar etc.

\subsubsection{Direct arginine growth}

The results of adding additional TEOS to a completed seed synthesis reaction mixture are shown in Figure 2.5. The maintenance of a monomodal size distribution, as well as the cube-root increase in diameter (Figure $2.5 \mathrm{~g}$ ), indicate that all of the additional silica was incorporated into the original particles rather than nucleating new ones.

The shape and surface morphology of the particles are worthy of note. In contrast to the familiar Stöber seeded growth process, the particles are not smooth spheres. The surfaces are rough to such an extent that the shape deviates significantly from spherical, even after more than doubling the seed diameter (Figure 2.5f). In a more positive contrast with the Stöber growth method, no particles were observed to have fused to form dumbbells or larger multiparticle aggregates.

Finally, Figure $2.5 \mathrm{~g}$ suggests that in the last two growth steps, the reaction was not completed. This was confirmed by the observation of an oily TEOS layer still present in the reaction flask. This may have been due to depletion of the arginine catalyst as it became incorporated into the particles. Furthermore, at this stage a deposit of silica was observed to be building up on the stirring bar. At this high silica concentration $(\sim 2 \mathrm{M})$, it was apparently not possible 
to maintain all of it in suspension and so the particles in Figure 2.5 are close to the limit of growth with this method.
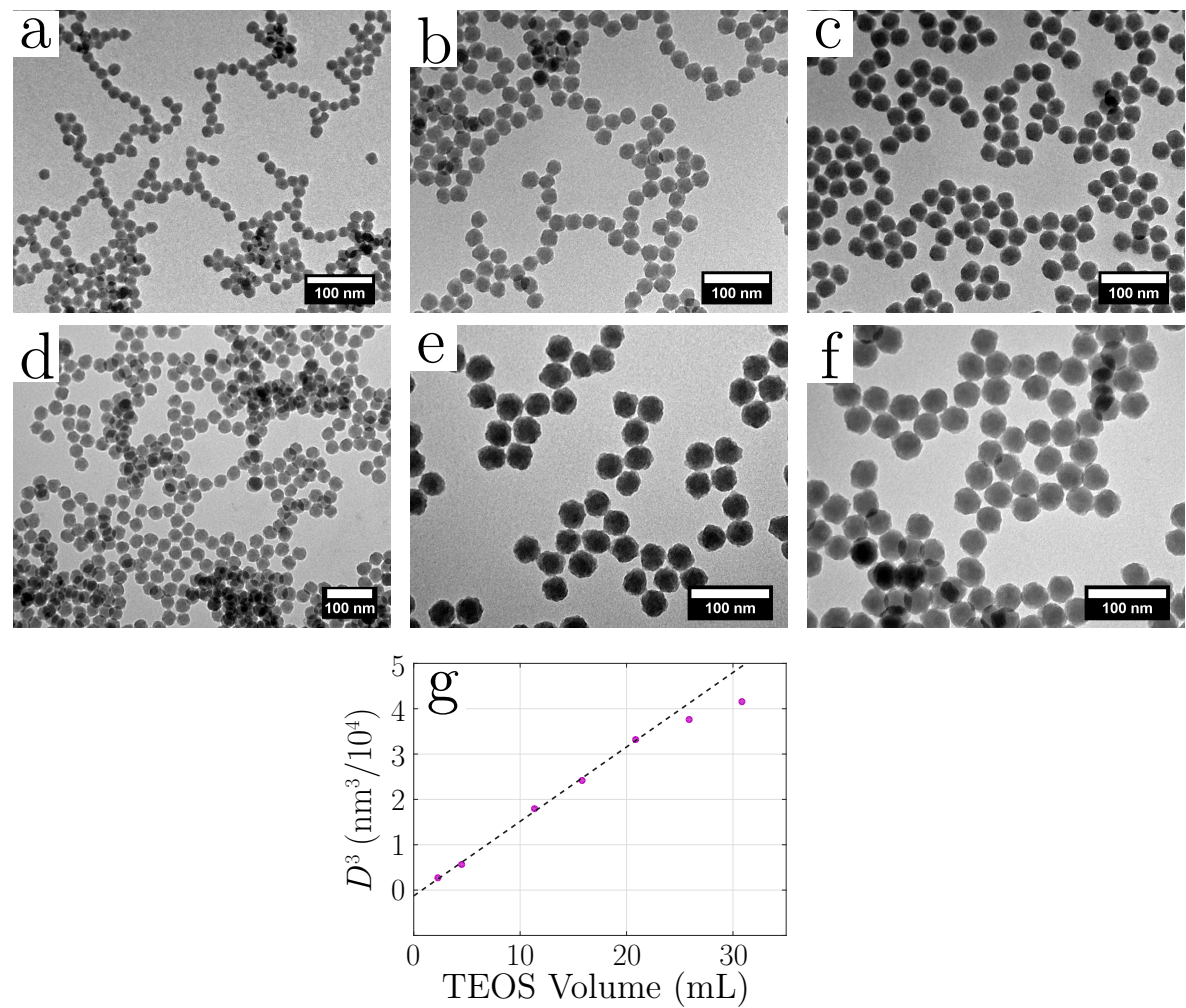

Figure 2.5: Direct growth of seed particles in Figure 2.4c to a diameter of ${ }_{6}^{35} 35 \mathrm{~nm}$. a-f) TEM images of the particles resulting from each growth step. g) Variation of the cubed diameter with the cumulative amount of TEOS added. The line is a fit to the first 5 points (seeds + four growth steps).

\subsubsection{Diluted arginine growth}

In order to take larger growth steps, the particle concentration was lowered by a factor of 10 while maintaining reagent concentrations of the seed synthesis. The particles resulting from this method are shown in Figures 2.6 and 2.7.

This method is similar to that utilised in Reference 29 in which monomodal 
systems of rough particles were made with diameters up to $120 \mathrm{~nm}$. Above this diameter, secondary nucleation occurred and was found to be unavoidable. A similar phenomenon was observed in the present work, with particles first grown to $83 \mathrm{~nm}$ without difficulty. These also had rough surfaces (as compared to Stöber particles, see e.g. Figure 2.8) and a monomodal diameter distribution after two growth steps (Figure $2.6 \mathrm{c}$ ). Rougher particles likely arise from addition of larger silica units throughout growth as discussed in Section 2.2.3 in the context of Stöber growth. Unlike in the Stöber case, in the two phase method the addition unit size remains constant so there is no smoothing effect of smaller addition units at the end of the reaction. It may be possible to find conditions in which the growth tends towards monomer addition to make smoother particles, but the aqueous reaction medium is less conducive to this than the homogeneous Stöber mixture. Here, the TEOS must be hydrolysed before it can move through the water phase and the energy benefit in assembling with a fellow monomer is greater in water than in ethanol.

At the subsequent growth step, aiming for $180 \mathrm{~nm}$, secondary nucleation occurred. Several attempts were made to avoid this and continue growth of the particles but as may be seen in Figure 2.7, none of these were successful in fully suppressing the appearance of secondary particles.

The attempted routes to avoid secondary nucleation were based upon the factors affecting the balance of nucleation and growth as depicted in Figure 2.2. First, lithium chloride salt was added to decrease the stability of the secondary particles (Figure $2.2 \mathrm{a}, \mathrm{k}$ ). In Figure $2.7 \mathrm{c}$, it may be seen that the proportion of secondary particles was reduced by this increased ionic strength. Unfortunately, further increase of the $\mathrm{LiCl}$ concentration did not suppress secondary particle formation completely but instead resulted in much more drastic and multimodal secondary particles (Figure $2.7 \mathrm{~d}$ ). The reason for this may lie in the delicate balance of colloidal stability involved in growth. For growth only, the main particles should be stable to each other and the protoparticles should be stable to each other but the protoparticles should be unstable with respect to the main particles, allowing them to add to the main particle surfaces. It may be that by further increasing the ionic strength, the protoparticles became unstable to each other, allowing many 'nucleation' events throughout the reaction, resulting in multimodal secondary particles.

Figure 2.7 e shows the particles resulting from growth with an increased concentration of $83 \mathrm{~nm}$ seed particles (an increased main particle surface area, Figure $2.2 \mathrm{n}$ ). This resulted in similar levels of secondary nucleation, suggesting that the issue is more one of stability than of the probability to encounter a seed surface to add to.

Finally, the arginine concentration was lowered in order to reduce the hydrolysis rate to lower the supersaturation of protoparticles and drive the balance 

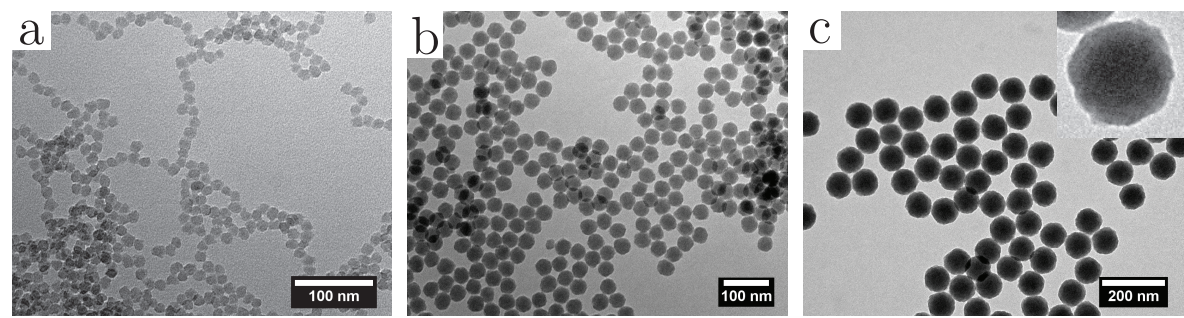

Figure 2.6: Successive growth steps of FITC dyed particles in water. (a) ${ }_{12}^{15} 15 \mathrm{~nm}$ (b) ${ }_{5}^{35} 35 \mathrm{~nm}$ (c) ${ }_{3}^{83} 83 \mathrm{~nm}$. The inset window has side length $100 \mathrm{~nm}$.

away from nucleation of new particles. As may be seen from Figure $2.7 \mathrm{f}$, the secondary nucleation instead became more severe. This could be for a number of reasons. First of all, the reduction in the arginine concentration also would reduce the ionic strength, increasing the stability of the secondary particles. Furthermore, the arginine plays a complicated role as a catalyst which is also partially incorporated into the final particles [68-70], making its contribution hard to predict.

\subsubsection{Immediate Stöber growth}

As the arginine growth yields particles with poorly defined surfaces in terms of structure (rough) and chemistry (variable arginine incorporation), the Stöber method was used to grow shells of well defined (and well studied [52]) silica around the seed particles. Stöber growth on arginine silica has been demonstrated previously, but only after some arginine catalysed seeded growth to $88 \mathrm{~nm}[29]$.

Stöber growth directly from the seed particles extends the accessible size range of smooth spheres downwards. In Figure $2.6 \mathrm{~b}$, the rough surfaces of the arginine grown particles are seen to significantly distort the particle shapes away from spherical for $35 \mathrm{~nm}$ particles. For the larger particles in Figure 2.6 c, the scale of the roughness is small compared to the $83 \mathrm{~nm}$ particle diameter, making the distortion less relevant for larger spheres. Thus for small diameters $(\sim 15-50 \mathrm{~nm})$, there is a need for a different method which results in surface roughness on a scale much smaller than these particles.

Unfortunately, these smaller particles are more difficult to grow without encountering either aggregation or the production of secondary particles. While consensus has not yet been reached on a mechanism, it is likely that the Stöber process proceeds at least in part by an aggregative growth mechan- 


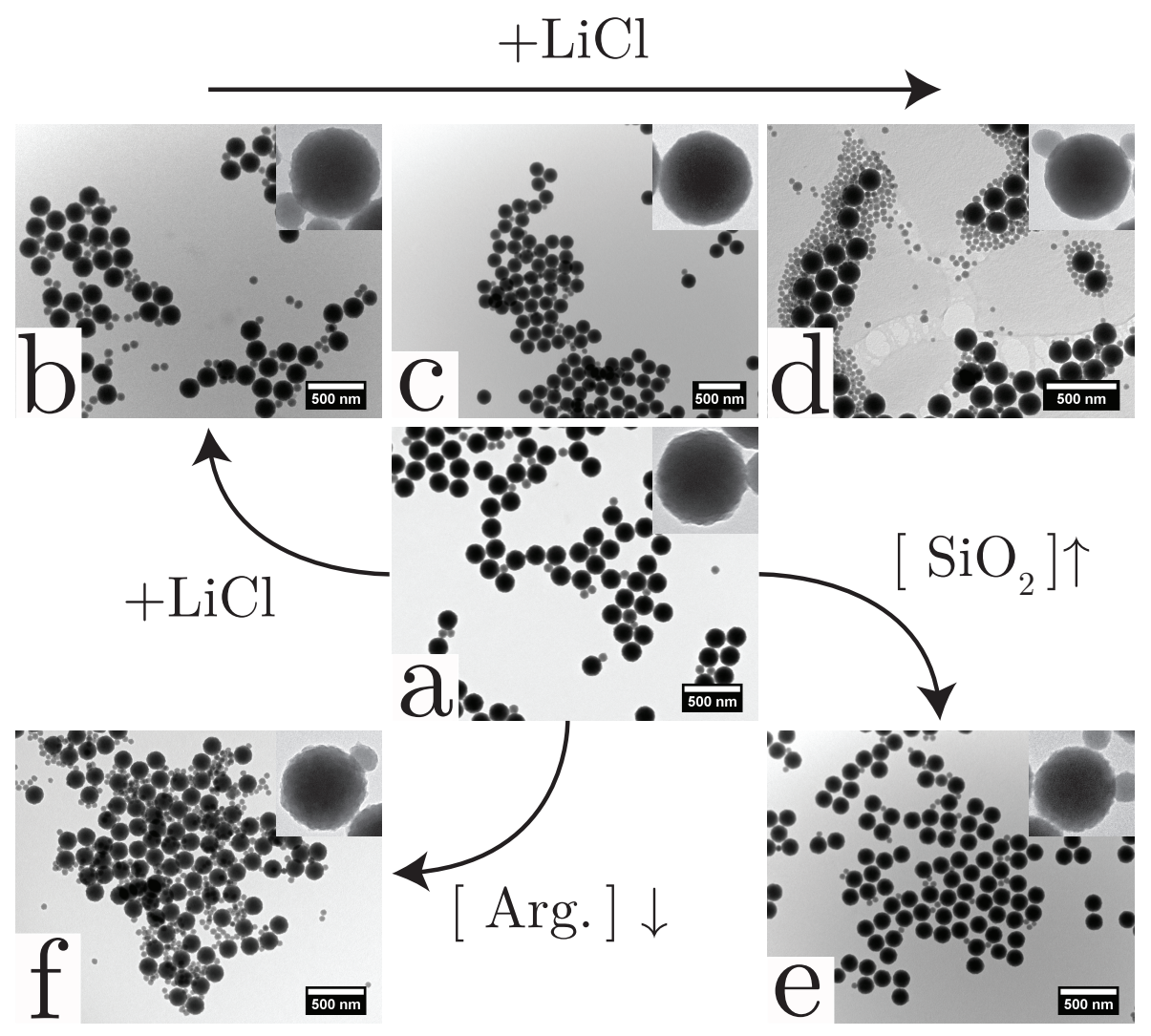

Figure 2.7: Attempts at growth of the particles in Figure 2.6 c to $180 \mathrm{~nm}$. (a) Big/small diameter: $182 / 83 \mathrm{~nm}$. (b) $182 / 76 \mathrm{~nm}$ (c) $177 / 75 \mathrm{~nm}$ (d) $166 / 51 \mathrm{~nm}$ (e) $156 / 58 \mathrm{~nm}$ (f) $166 / 54 \mathrm{~nm}$. Insets show a single particle at larger scale in order to make surface roughness visible. All inset windows have side length $200 \mathrm{~nm}$. 
ism $[25,52,54]$. Therefore the increased difficulty of growing smaller nanoparticles may be understood in terms of the relative stability of the seeds and the addition particles. For aggregative growth of single particles, it is required that the small addition particles be unstable and the larger seeds are stable. Within the colloidal size range, larger particles are generally more stable $[71,72]$. This means that the closer in size the seeds are to the addition particles, the narrower the stability range in which single particle growth is possible.

In practice, the principal factor controlling stability in this reaction is the ionic strength. Figure 2.8 shows the results of carrying out the synthesis at increasing ionic strength. When it was low, the addition particles were too stable and formed stable secondary particles. When it was high, electrostatic screening destabilised the seed particles causing them to aggregate into chains, becoming permanently bonded together by the silica growth. The reason for forming chains rather than fractal aggregates of higher dimension is due to electrostatic effects. Namely as soon as a dimer is formed, the energy barrier for an approaching monomer is lower at the ends than at the sides [73]. This phenomenon has been well studied with gold nanoparticles [73-75] but has also been observed for arginine catalysed seeded growth at high ionic strength [44].

In this reaction, the ionic strength is principally determined by the added ammonia catalyst, both in being an ionic species itself and by controlling the rate of TEOS hydrolysis. With an increased rate of hydrolysis, the initial peak in conductivity is heightened as discussed in Section 2.2.1. Thus the ammonia concentration was adjusted to achieve the differences in Figure 2.8. In the case of aggregation, the probabilities of a seed being in an aggregated chain of length 1-8 particles was computed by counting the single particles and aggregates in TEM images. Figure $2.8 \mathrm{~d}$ shows how the mean value of the chain length approaches 1 (i.e. all single spheres, the desired outcome) with decreasing ammonia concentration, seemingly in an exponential fashion. The dependence of the chain length on the ionic strength corresponds to a changing aggregation rate. In terms of DLVO theory, the aggregation rate should increase super exponentially at salt concentrations below complete double layer suppresion [76]. Therefore it is concluded that the observed dependence is in qualitative agreement with DLVO predictions, without a more in depth attempt to apply the theory to this complicated reaction system.

It is worth noting that ammonia plays two roles in the particle stability. The basic environment increases stability by deprotonating the silica surface and hence increasing its negative charge. In fact, the small seed particles are not stable in ethanol and were observed to rapidly flocculate when added to the pure solvent. For this reason, it is crucial when carrying out the described growth that the reaction mixture is basic before the seeds are added. Incidentally, this 


\section{Synthesis of monodisperse fluorescent silica nanospheres}

is also thought to be the reason for the formation of strings of particles in the TEM images of the seeds (Figure 2.4), since the particles were dropcast from ethanol before imaging.

Another factor which is often discussed in literature is the age of the seed particles [29, 69]. The consensus seems to be that seeded growth is not possible with older seeds. In this work the age of the seeds was found to have no effect on seeded growth (neither Stöber nor arginine catalysed). Similar results were observed with newly synthesised seeds and seeds which were stored in the reaction mixture for more than one month. In Reference 29, it was noted that "it seems that aged particles partially aggregate upon addition of ammonia, yielding polydisperse samples upon regrowth". It may be in that work the ammonia was not always added first to the reaction mixture (as discussed above) and the resulting aggregation was attributed to aged seeds. It is also possible that some aspect of the storage conditions was different between that work and this one.

Further seeded Stöber growth was carried out when the correct conditions were found and the results are shown in Figure 2.9. While the particles are generally smooth and monodisperse, it may be seen that the size range is still one where stability is an issue. This is evidenced by the few aggregated and secondary particles in Figures $2.9 \mathrm{a}$ and b respectively.

\subsubsection{Early (dyed) Stöber growth}

One way to incorporate dye into the particles is to add a Stöber-like silica shell in which a fluorescent label is covalently bonded to the silica matrix. This addition of a dyed shell was done from seeds grown a little larger to $33 \mathrm{~nm}$ using the arginine method. The series showing the seeds, first Stöber shell, dyed shell and subsequent spacer shells is shown in Figure 2.10. The synthesis was demonstrated with two dyes: RITC (Figure 2.10a-e) and FITC (Figure 2.10 f).

The first thin Stöber shell was added since both the switch from water to ethanol of the arginine seeds and the dying procedure are sources of potential instability and hence aggregation. By first growing an undyed shell, these two factors were at least not present at the same time. It may be seen that the first shell growth yielded single particles but the dying step resulted in some multiparticle aggregates. Likely some parameter (ionic strength) tuning would be able to reduce the occurrence of these aggregates.

The final two steps do not appear to have introduced new aggregates, but those created during the dying step are of course still present.

In order to demonstrate the utility of these dyed particles for single particle studies, colloidal crystals were assembled and imaged with $3 \mathrm{D}$ STED microscopy. Figure 2.11 shows slices through the image volume as well as fitted 

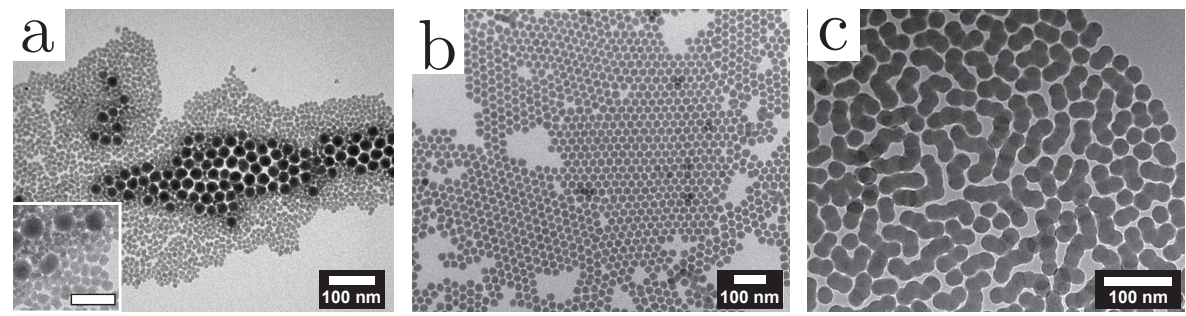

Increasing Ionic Strength

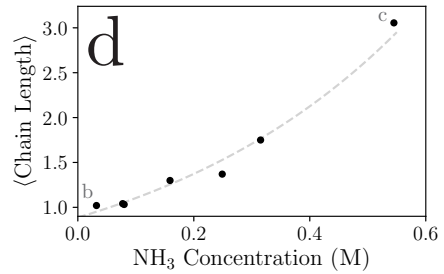

Figure 2.8: Particles grown directly with Stöber silica. a) At low ionic strength, stable secondary particles were formed. Big/small particle diameters: $\sim 28 \mathrm{~nm} / 13 \mathrm{~nm}$. Inset shows ill defined shape of secondary particles at higher magnification (scale bar $50 \mathrm{~nm}$ ). b) At intermediate ionic strength the particles grow as intended, here to ${ }_{7}^{15} 27 \mathrm{~nm}$. c) At high ionic strength, strings of aggregated particles were formed. d) Mean length of aggregated chains (in number of seed particles) as a function of the ammonia concentration. The systems which yielded secondary particles rather than aggregated chains are not shown. The first and last points correspond to the systems in (b) and (c) as indicated in the plot. The dashed line shows the equation $\langle L\rangle=0.9 \exp \left(2.2\left[\mathrm{NH}_{3}\right]\right)$.
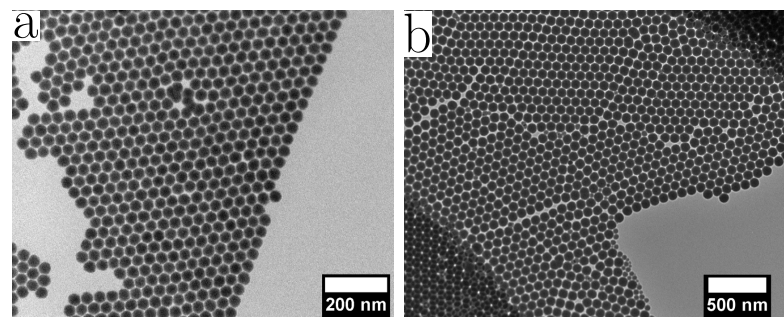

Figure 2.9: Particles grown larger with Stöber silica from those depicted in Figure 2.8 b. a) ${ }_{5}^{15} 39 \mathrm{~nm} \mathrm{b)}{ }_{4}^{15} 79 \mathrm{~nm}$. 


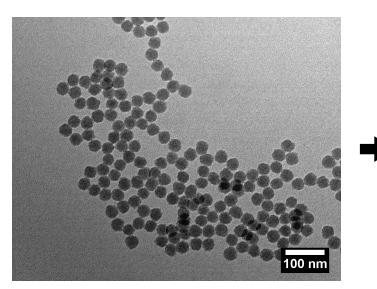

(a) ${ }_{8}^{33} 33 \mathrm{~nm}$

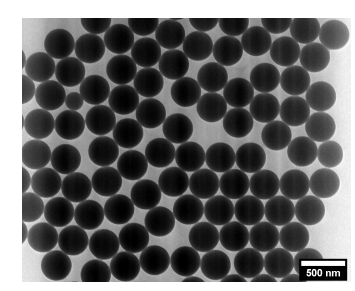

(f) ${ }_{2}^{34} 349 \mathrm{~nm}$

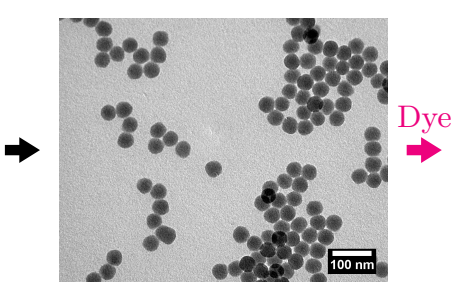

(b) ${ }_{5}^{33} 41 \mathrm{~nm}$

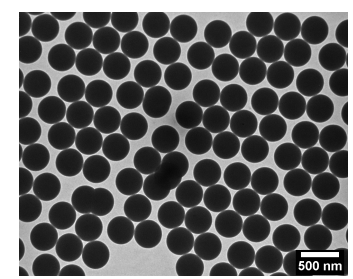

(e) ${ }_{2}^{33} 359 \mathrm{~nm}$

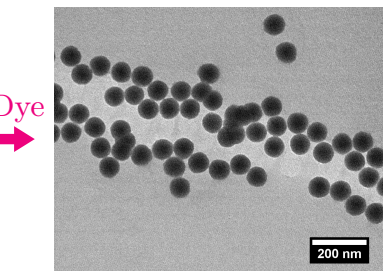

(c) ${ }_{4}^{33} 77 \mathrm{~nm}$

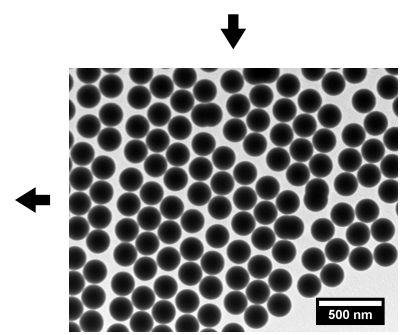

(d) ${ }_{2}^{33} 200 \mathrm{~nm}$

Figure 2.10: Particles with fluorescent dye covalently incorporated via modified Stöber method. a) Particles resulting from single diluted arginine seeded growth step. Comparable to Figures $2.3 \mathrm{f}$ and $2.6 \mathrm{~b}$. b) Single Stöber growth step. Steep decrease in polydispersity likely in part due to increase in sphericity. c) Particles after growth of RITC dyed shell. It may be seen that this is the step most vulnerable to aggregation during growth. d, e) Further Stöber growths to provide a spacer between the fluorescent 'cores'. f) Particles grown with the same method but with FITC dye incorporated. 
particle locations in $3 \mathrm{D}$.

\subsubsection{Late Stöber growth}

If fluorescent dye is not a requirement, the most convenient route to $350 \mathrm{~nm}$ silica nanoparticles is that depicted in Figure $2.12 \mathrm{a}-\mathrm{c}^{8}$. Growth to around $80 \mathrm{~nm}$ with the arginine method is simple and robust and sufficiently large that the switch to Stöber growth does not give rise to the stability issues discussed in previous sections. Thus it may be seen in Figure $2.12 \mathrm{c}$ that the particles are very well defined smooth spheres.

Finally, in the bottom row of Figure 2.12, it is shown that it was possible to remove the secondary particles from the larger arginine growths and add a Stöber shell. While the main particles are well defined, some secondary particles are still visible which have been grown and therefore the method described above is preferred.

\subsubsection{Decreasing polydispersity upon seeded growth}

Figure 2.13 shows the rate of polydispersity decrease for all the particles in this chapter. There is considerable spread in the data, making it difficult to draw conclusions. The spread comes from the fact that this study was not carried out with the intention of being a detailed kinetic one. For this reason, the polydispersities were not measured with high precision: only $100-200$ particle diameters were measured 'manually' for each particle system. Furthermore, the particle diameters were not measured in TEM images at maximal magnification, meaning that a small but systematic contribution to the polydispersity was made by measurement error. While the mean diameter may be trusted as random measurement errors will balance on either side of the average, all measurement errors make a positive contribution to the apparent polydispersity. This is particularly problematic for low polydispersities on the order of $1 \%$.

Examining the data nonetheless, $\gamma \approx-0.6$ was found for both the amino acid and Stöber type growths, corresponding to $\alpha \approx 2.4$. It is known that $\alpha$ should be close to 2 for Stöber growth, so this high value would seem to be the result of systematic overestimation of the polydispersity, casting doubt on the accuracy of the values resulting from both growth types. Another factor which would drive $\alpha$ up in the Stöber case is the surface roughness (for the amino acid case the roughness is on the scale of the monomers). However, none of the particle surfaces observed in this chapter appear to exhibit a fractal dimension as high as 2.4.

\footnotetext{
${ }^{8}$ In fact there is some dye in these particles but the fluorescent volume of the dyed core is so small compared to the bulk of the particle that it is inconsequential.
} 

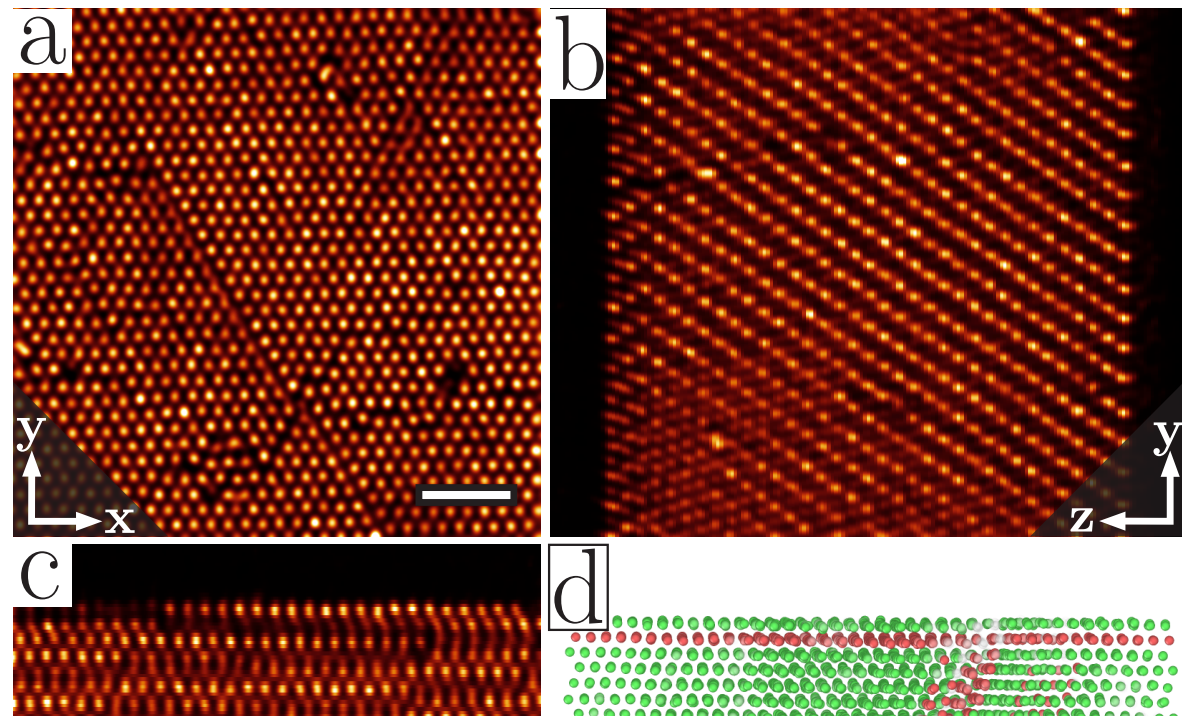

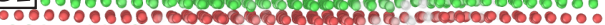

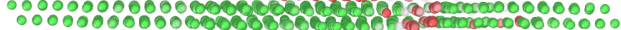

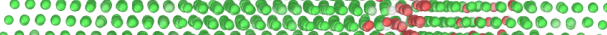

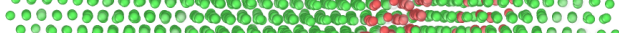

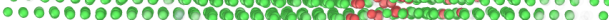

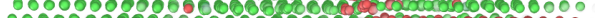

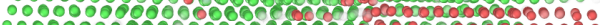

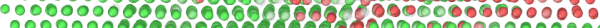

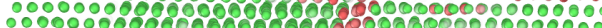
0000000000000000001000000000000000 000000000000000000000000000000000000 0000000000000000000000000000000000000 (1)

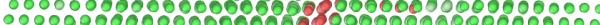
0000000000000000000000000000000000 .0000000000000000000000000000000000000 - 000000000000000000000000000000000

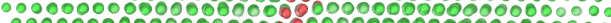
 10000000000000000600000000000000000 00000000000000086000000000000000 Z 00000000000000000000000000000000000

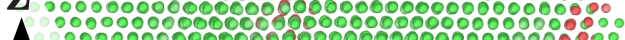

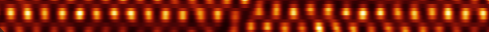
Z
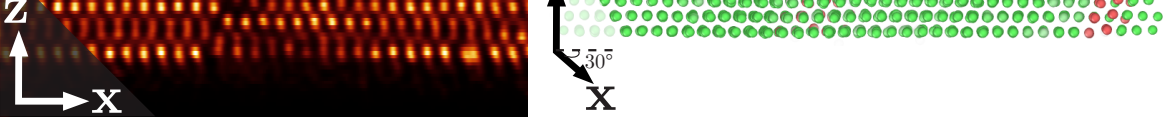

Figure 2.11: 3D STED microscopy of a colloidal crystal composed of ${ }_{2}^{33} 359 \mathrm{~nm}$ particles. $\mathbf{a}-\mathbf{c}$ ) Orthogonal slices through the deconvolved image volume showing that the fluorescent cores are resolvable in all three dimensions. The scale bar has length $2 \mu \mathrm{m}$. d) Rendering of the imaged volume based on fitted particle coordinates. The particles are coloured according to their identified crystal environment (FCC: green, HCP: red, unidentified: transparent). This makes the grain boundary (visible in a,c) as well as the stacking fault near the top (visible in b,c) clear and demonstrates the utility of this model system for crystallographic studies. 


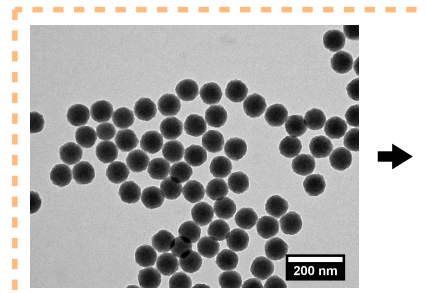

(a) ${ }_{3}^{83} 83 \mathrm{~nm}$
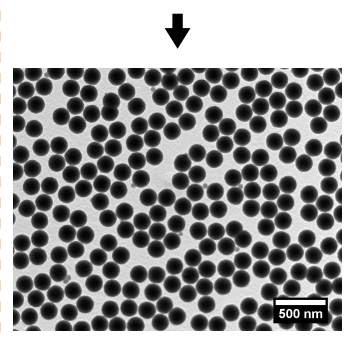

(d) ${ }_{2}^{665} 165 \mathrm{~nm}$

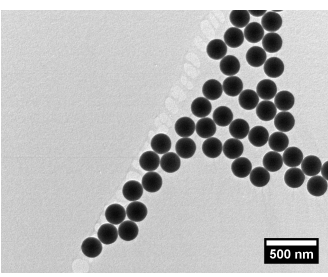

(b) ${ }_{2}^{83} 201 \mathrm{~nm}$

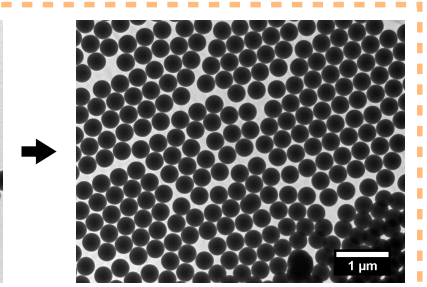

(c) ${ }_{1}^{83} 350 \mathrm{~nm}$

Figure 2.12: Effectively undyed particles obtained by 'late' switch from arginine-catalysed to Stöber seeded growth. Note that this figure is an enlarged extract of the lower boxed region of Figure 2.3. a) Particles grown to $\varnothing 83 \mathrm{~nm}$ with the arginine catalysed method. b, c) Successive Stöber growth steps of these particles. d) Particles obtained by arginine growth of those in (a) followed by removal of most of the secondary particles by centrifugation. The unwashed particles are shown in Figure 2.7 d. e) Further Stöber growth of the particles in $(\mathrm{d})$. 

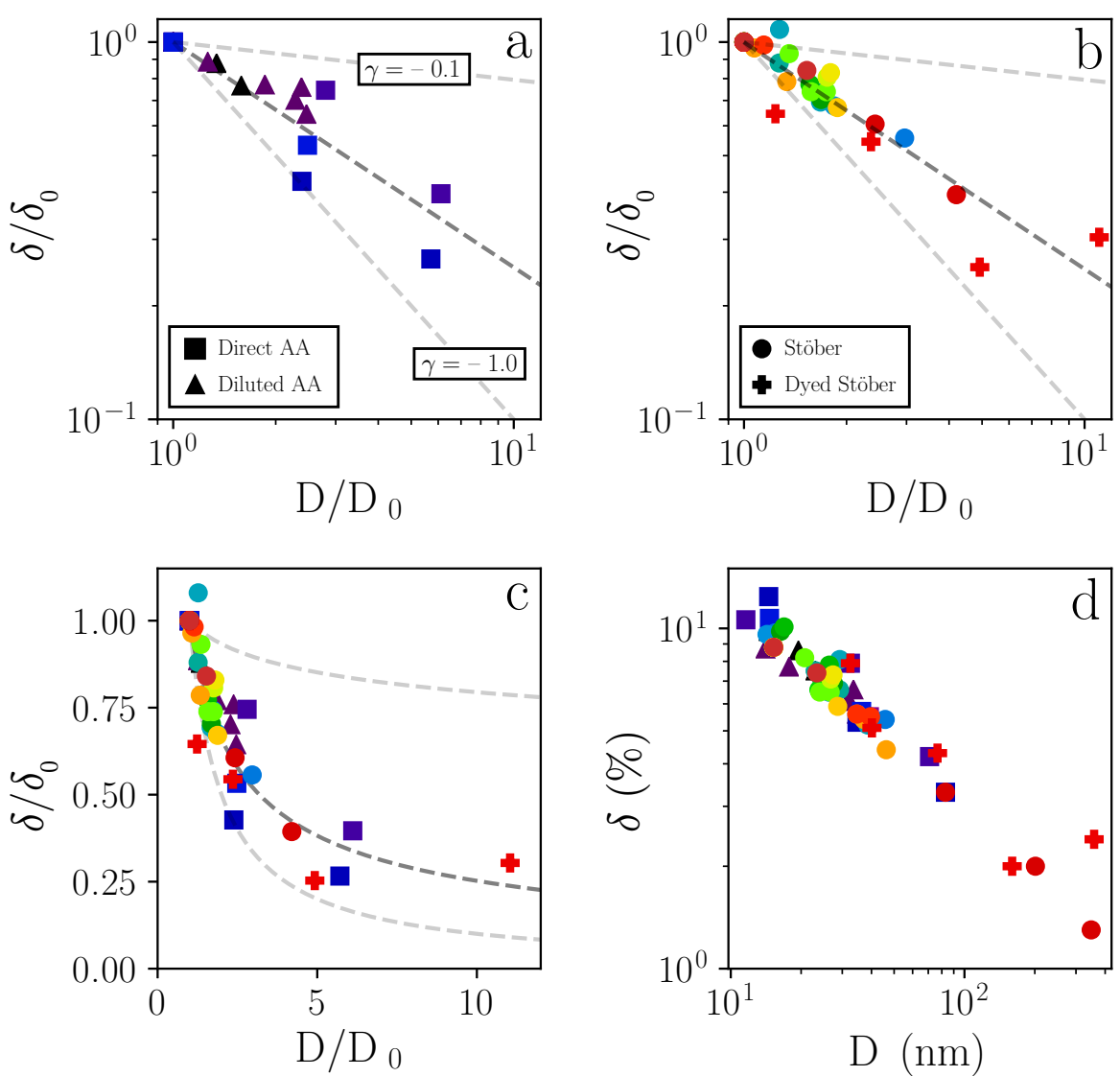

Figure 2.13: Behaviour of the polydispersity during seeded growth. a) Amino acid catalysed growth (AA). The fit line (dark grey dashed) corresponds to $\gamma=-0.6$. Both the diameter and polydispersity are normalised with the values of the seed particles for each series $\left(D_{0}\right.$ and $\left.\left.\delta_{0}\right) \mathbf{b}\right)$ Stöber growth. The fit line corresponds to $\gamma=-0.6$. c) Data points from (a) and (b) with linear scales. d) The raw (not normalised) data used to construct the plots in this figure. The symbol shapes distinguish specific growth situations and each growth series is assigned a colour. The symbols correspond to the same particle systems in all four plots. The upper and lower light grey dashed lines in $(\mathrm{a}-\mathrm{c})$ show the behaviour with reference $\gamma$ values of -0.1 and -1.0 respectively. 

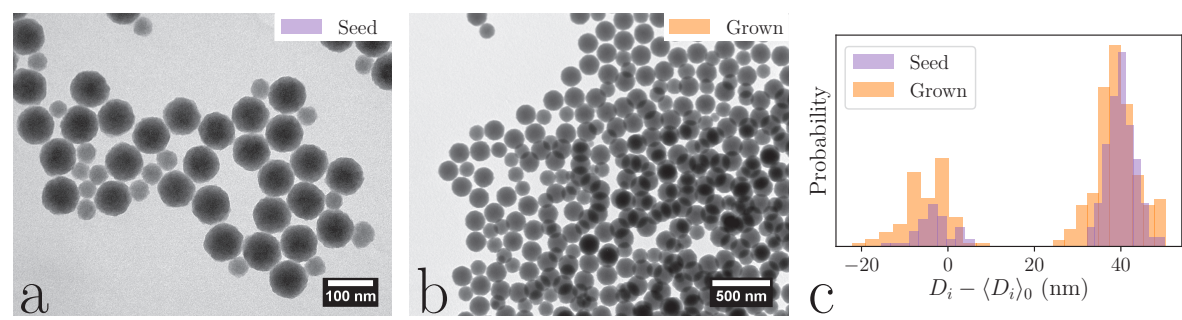

Figure 2.14: Competitive amino acid growth of bidisperse seeds. a) Seed particles with diameters ${ }_{10}^{44} 44 \mathrm{~nm}$ and ${ }_{4}^{86} 86 \mathrm{~nm}$. b) Grown particles with diameters ${ }_{4}^{134} 134 \mathrm{~nm}$ and ${ }_{3}^{177} 177 \mathrm{~nm}$. c) Histograms of the distributions. The horizontal axis shows the diameters of the four distributions (small seeds, big seeds, small grown, big grown) with the mean diameter of the corresponding seeds subtracted. $i$ indicates the 'small' or 'big' particle distribution.

As this work was focussed on monodisperse particles, growth was generally not done after the system became bidisperse. However, one well-defined bidisperse system of the type discussed in Section 2.4.3 was grown with the amino acid method. The seeds and resulting particles are shown in Figure 2.14. Inserting the mean diameters into Equation 2.4 and solving numerically for $\alpha$ yields $\alpha=2.0$. As discussed above, this value corresponds to surface reaction limited growth, in which the absolute difference between the particle sizes remains constant. This is visualised in Figure $2.14 \mathrm{c}$, in which the two distributions lie on top of each other after subtracting the mean diameter of the seeds, because both particle sizes increased by the same absolute value. Equation 2.3 also serves as a quick check for whether or not $\alpha=2$ holds, since in that case it simplifies to $\Delta D_{s}=D_{s}-D_{0 s}=\Delta D_{b}=D_{b}-D_{0 b}$. For the particles in Figure 2.14, $\Delta D_{s}=90 \mathrm{~nm}$ and $\Delta D_{b}=91 \mathrm{~nm}$.

While this single run of bidisperse growth is not sufficient to draw strong conclusions, it does suggest that the amino acid growth proceeds in a surface reaction limited manner. This means that the true polydispersity is expected to decrease in proportion to $1 /\langle D\rangle$. Thus it appears that the growth kinetics of the Stöber and amino acid processes are similar, even though the mechanisms are different.

\subsubsection{Surface Treatment}

The versatility of silica nanoparticles is increased by the ability to modify the particle surfaces and thus allow them to be dispersed in non-polar solvents. 
This was demonstrated in this work by coating the particles with stabilising $\mathrm{C}_{18}$ chains using the silane coupling agent O T M OS. The effective diameter of the particles is slightly increased by the coating.

Figure 2.15 a shows the particles before the surface treatment. These are the same particles as shown in Figure 2.9 a, but after some purification steps to remove the worst of the aggregates. After organic coating, the particles were dispersed in cyclohexane and deposited upon a (polar) liquid-air interface [77] before imaging the resulting assemblies in TEM (Figures $2.15 \mathrm{~b}$ and $\mathrm{c}$ ).

The success of the coating is demonstrated by the formation of colloidal crystals from cyclohexane. Crystallisation requires the particles to be individually stable in order to sample sufficient configurations and reach the thermodynamically favoured crystal. A new feature which is observed in the coated particles is the appearance of Moiré patterns in regions of multiple layers. This phenomenon is observed when two periodic patterns are overlayed with a rotational and/or translational offset. This indicates that the particles have formed a stable film upon assembly and then two of these have fallen on top of each other when scooping the film from the interface onto a TEM grid. The ability to form free standing films has previously been observed in nanoparticles coated with hydrocarbon chains and is due to van der Waals attraction between interdigitated chains on adjacent particles [77, 78].

Thus it has been shown that these particles may be readily transferred into organic solvents which allows them to be used for studies of, for example, supraparticle assembly as shown in Figure 2.16. These were made by allowing cyclohexane droplets containing the dispersed nanoparticles to shrink by dissolution and evaporation in water.

\subsubsection{Efficacy of the dye in different systems}

Almost all of the particles in this chapter were labelled with a fluorescent dye. Depending on the method of incorporation and the particle diameter, the labelling is suitable for different purposes.

In the case of the smallest particles, only the $15 \mathrm{~nm}$ 'arginine silica' core was dyed and white Stöber silica was grown around it (Section 2.4.4). It may be seen in Figure $2.16 \mathrm{~d}$ that the dye inside the core of these $39 \mathrm{~nm}$ particles was sufficient to image dense ensembles of the particles and discern the shapes of such ensembles. Single particle imaging of such small particles in dense suspension is not possible using optical microscopy. In more dilute suspension, or equivalently in particles grown larger with white spacer silica, it turned out that the signal from the tiny fluorescent core was insufficient to image the particles individually. Even when further dye was (presumably) incorporated during seeded arginine growth (Section 2.4.3), the signal was still insufficient 

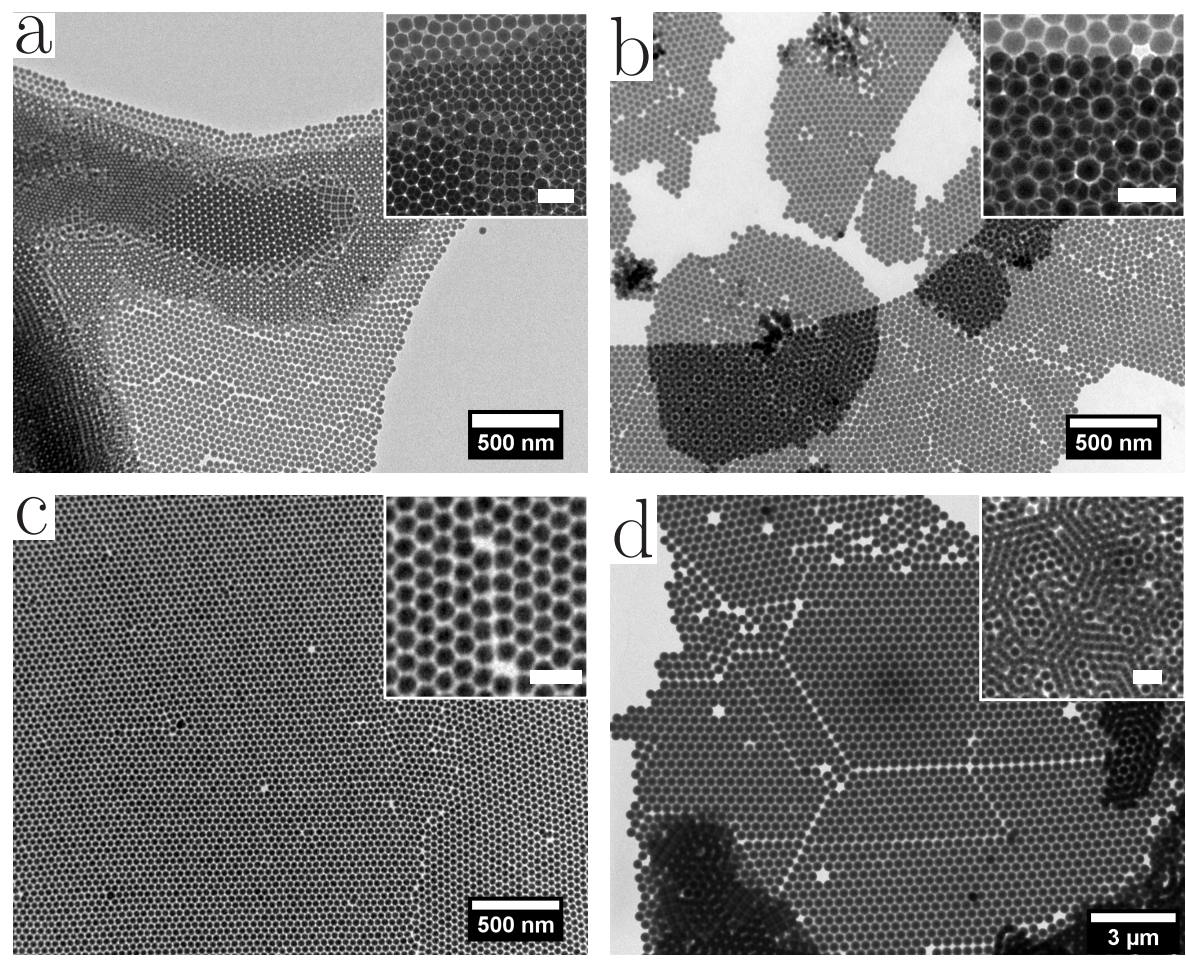

Figure 2.15: Sterically stabilised silica nanoparticles. a) ${ }_{5}^{15} 39 \mathrm{~nm}$ particles before surface treatment. Inset: enlarged central region showing stacking up to three layers. b) Monolayers of the $\mathrm{C}_{18}$ coated particles assembled on ethylene glycol surface. The Moiré patterns (enlarged in inset) are characteristic of multiple stable layers stacked with a small offset [79]. c) Monolayer assembled on a diethylene glycol surface. The few remaining aggregated particles are clearly visible where they disrupt the lattice. The inset shows a dumbbell and its effect on the surrounding lattice. d) Larger $\left({ }_{1}^{83} 350 \mathrm{~nm}\right)$ particles coated in the same way and assembled by dropcasting from cyclohexane. Here the inset shows a different region with another Moiré pattern. The inset scalebars are $100 \mathrm{~nm}$ (a-c) and $1 \mu \mathrm{m}(\mathrm{d})$. 

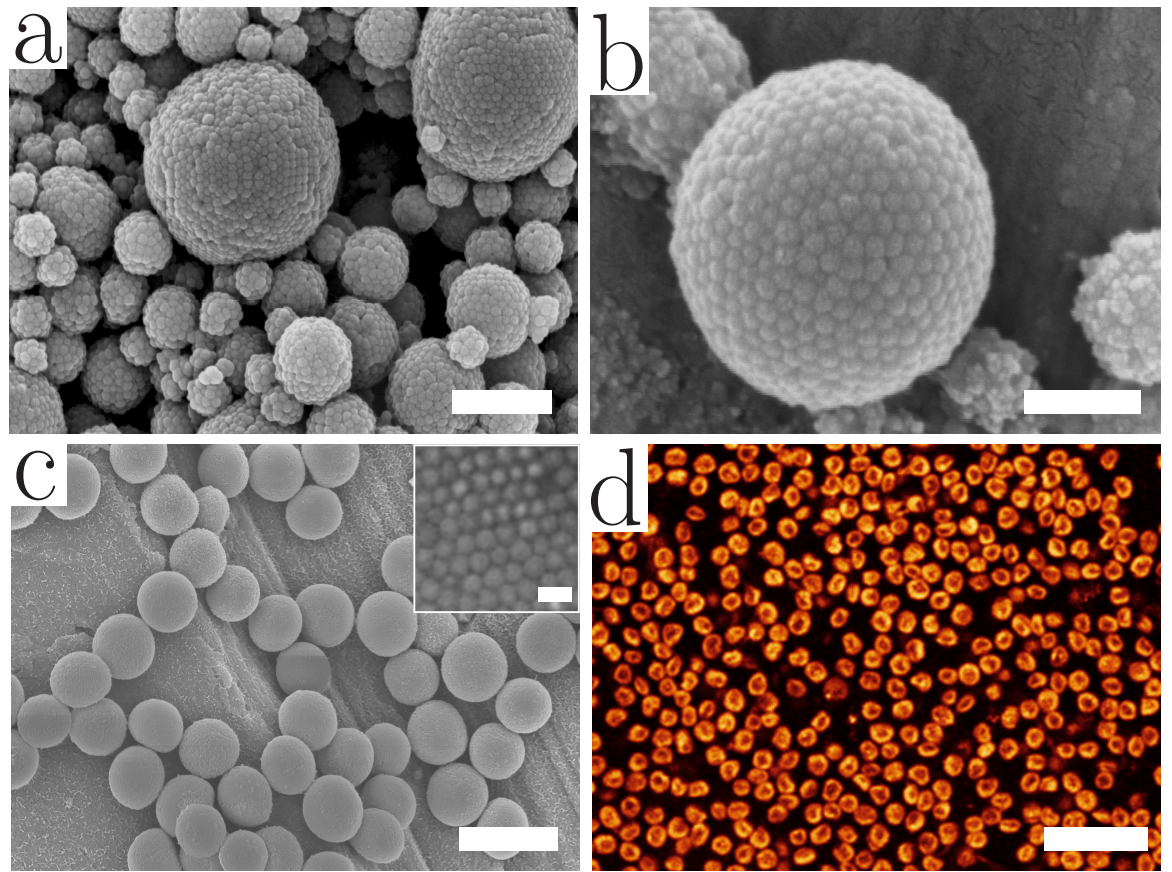

Figure 2.16: Supraparticles assembled from sterically stabilised particles shown in Figure 2.15 b,c. a) Polydisperse supraparticles resulting from poorly defined emulsion created by hand shaking. b) Higher magnification image of same sample. c) Low polydispersity supraparticles resulting from microfluidic emulsification. The average diameter is $1.1 \mathrm{\mu m}$ and the polydispersity $9 \%$. The inset shows a the same particles in a colloidal crystal, but with poor image quality due to surfactant deposition (insufficient washing). d) Confocal image of supraparticles formed from a similar emulsion to that in (c), but with much more rapid droplet shrinking causing buckling into coffee bean like shapes. Scale bars are a) $500 \mathrm{~nm}$, b) $200 \mathrm{~nm} \mathrm{c)} 2 \mu \mathrm{m}$ (both) and d) $10 \mu \mathrm{m}$. 
for imaging.

The mechanism of dye incorporation in the amino acid method is not yet fully understood. It is known that addition of an isothiocyante dye during the Stöber process does not yield fluorescent particles [27]. Only by adding a silane coupling agent can the dye be incorporated. Therefore it is clear that the amino acid has a role to play. It has been suggested that the isothiocyanate group attached to the dye becomes covalently bonded to the amine group of the amino acid $[80,81]$. The amino acid is known to become non-covalently incorporated into the silica matrix during the synthesis [68]. It seems likely that the dyebound amino acid molecules are able to do the same. With the addition of capping silica shells, the physisorbed dye groups may be permanently trapped inside the particles [29]. Further understanding of this mechanism is required in order to optimise the dye loading using this method.

In this work, additional dye was added into the larger particles using the established method of covalently bonding an isothiocyanate dye to the silica matrix in a modified Stöber process. With this additional fluorescent shell and a sufficiently large spacer shell grown around it, it was possible to image the single particles in $3 \mathrm{D}$.

\subsection{Conclusions}

Synthetic routes to uniform spherical silica nanoparticles with diameter in the range $30-360 \mathrm{~nm}$ with various properties suitable for different purposes were demonstrated. These involved growth of Stöber silica on particles made by the newer arginine catalysed method. In this way, smooth spherical particles with the surface chemistry of Stöber silica could be made at sizes lower than previously achieved. All of the particles were fluorescently dyed allowing them to be used as model systems for study with optical microscopy.

At the low end of the size range, the dye incorporated in the amino acid synthesis was shown to be suitable for imaging ensembles of particles. When the particles were grown larger, the dye incorporated in this way was no longer sufficient and so a method was optimised to bind further dye into the silica with a silane coupling agent and a modified Stöber growth. This resulted in a particle system with a diameter of $360 \mathrm{~nm}$ (and gravitational length $\sim 50 \times$ greater) which could be imaged in $3 \mathrm{D}$ as single particles.

In order to understand the behaviour of the polydispersity during amino acid catalysed seeded growth, the changes in the size distribution were examined for monomodal and bimodal distributions. In the former it was found that the measurement error in the polydispersity was too high do draw conclusions. For a bidisperse system, preliminary results suggest that the amino acid growth is 
surface reaction limited and the polydispersity may be expected to decrease in inverse proportion to the mean diameter.

Finally, by modifying the surface of the particles, they could be dispersed in non polar organic solvents. Thus the model system presented was shown to be tunable in both size and surface interactions, as well as having particles minimally affected by gravity and yet suitable for single particle imaging in dense suspension.

\subsection{Outlook}

The next step in this research is to demonstrate the utility of the particles in a more extensive study of colloidal phase behaviour. This might take the form of a nucleation study or of answering fundamental questions about the glass transition.

From a synthetic perspective, a particle system can always be improved. In this case, further optimisation could reduce the formation of dumbbells to none at the silane coupled dying step. This dying step could also be carried out on smaller particles, to shrink the diameter of the fluorescent core and hence final particle diameter in order to further increase the gravitational length.

To improve understanding of the growth mechanism and kinetics, further studies of competitive seeded growth of bidisperse particles would be the most effective route.

Further characterisation of the particles would also be beneficial. In particular, an in depth study of the dye loading of the different particle systems in this chapter would facilitate improvement of the fluorescent signal from the particles and hence more effective $3 \mathrm{D}$ microscopy.

\section{Acknowledgements}

This work was carried out in collaboration with Relinde van Dijk-Moes and Judith Wijnhoven. The particles in Figure $2.10 \mathrm{f}$ were made by Roy Hoitink. Wendy Steinvoort, Anan Mohammed and Michael Ludwig provided valuable feedback after using the methods described in this chapter. All are gratefully acknowledged for their contributions. 


\title{
3 The Nanopede: Parallelised microfluidics for colloidal droplets
}

\begin{abstract}
Droplet microfluidics is well known to be capable of producing emulsion droplets of very low polydispersity, albeit with low throughput. Parallelisation has been successfully applied to scale up the production of monodisperse droplets but this has not yet been achieved for the full range of droplet sizes which are accessible in single droplet junctions. Crucially, the colloidal size range with droplet diameters around $1 \mu \mathrm{m}$ (or less) is still missing. In this work, a new device is tested which is based on the downscaling of a recently presented parallel design known as the 'millipede'. This 'nanopede' was found to yield crystallising droplets of close to $1 \mu \mathrm{m}$ with polydispersity below $7 \%$. Preliminary results are also presented in which this device is used for the formation of new colloidal particles by polymerising the droplets or dispersing nanoparticles within them to yield supraparticles after removal of the dispersed liquid.
\end{abstract}




\subsection{Introduction}

Microfluidic devices are the artisans of the emulsification business. While droplets can be made by the billion per passing moment by other mechanical techniques $^{1}$, none can match the size uniformity achieved by the individual and sequential crafting of each droplet at the microfluidic junction. Droplet microfluidics as a field may be traced back to the 1960 s with the invention of the inkjet printer $[85,86]$. In the decades since, progress has been driven by the parallel development of lab-on-a-chip devices and carried by innovations in microfabrication from the semiconductor industry. However, despite this long period of incubation and the continued predictions of revolutionary changes brought in by microfluidic technology, the field has not yet reached maturity $[86,87]$.

The reasons that microfluidic devices in general are still largely confined to academic research laboratories are varied but there is a recurring theme: the difficulties in scaling. Microfluidic devices often operate in a serial manner, with the droplet maker being the archetype. The oft quoted solution is to simply run many droplet making chips in parallel but this only shunts the scaling problem a little further down the road. Namely to the scaling of production. The fabrication techniques used in prototyping are frequently unsuitable for mass production [86], meaning that commercially available microfluidic devices are expensive (a few hundred euros for a device containing a single dropmaking junction). Running a thousand such devices in parallel is simply not feasible, neither economically nor practically.

Thus attention has increasingly turned to on-chip parallelisation, allowing many drop makers to be supplied by a few interfaces with the world. From the many proven droplet forming geometries, came many different attempts to parallelise them [88]. These may be broadly categorised into those in which droplet formation is controlled by external pressure, and those in which it is governed by the Laplace pressure. Here the discussion will be limited to the latter which have the advantage that pressure differences in the device (spatial or temporal) should not cause significant changes to the produced droplet size.

Such devices belong to a broad class utilising step emulsification ${ }^{2}$ in which droplet formation is driven by the Laplace pressure difference when the shallow dispersed phase channel meets a deep emulsion channel in which the pressure is much lower. This was pioneered in the 1990s with arrays of straight microchannels leading to a wide but still of low height 'terrace region' followed by a 'step' to a much deeper region [89, 91]. Many papers have been published

\footnotetext{
${ }^{1}$ Examples include membrane emulsification [82], Taylor-Couette shearing [83], high pressure homogenisation [84] and splashing around while washing dishes.

${ }^{2}$ Also known as terrace emulsification [89] and edge droplet generation (EDGE) [90].
} 
based on this design ${ }^{3}$ and by 2007 a range of droplet sizes from $1.4-100+\mu \mathrm{m}$ had been created in these highly parallelised devices [93, 94], although the smallest of them with the relatively high polydispersity of $17 \%$ [93]. At the lower end of the size range, droplets with diameter below $\sim 5 \mu \mathrm{m}$ fall into the colloidal size range, and can therefore act as a new colloidal model system if they are sufficiently monodisperse.

As these small droplets are of particular interest to the field of colloid science, it is worth comparing how this parallelised production compares to previously available small droplet makers. Unsurprisingly, single droplet makers are still able to produce smaller and more monodisperse droplets. By using a combination of capillary focussing and a step, highly monodisperse $2.5 \mu \mathrm{m}$ diameter droplets have been made and used to study colloidal crystals [95]. Shortly afterwards, the range of these devices was extented to cover much of the colloidal size range: from $400 \mathrm{~nm}$ to $3.5 \mu \mathrm{m}[96,97]$. Unfortunately, these impressive devices rely on precise control of both the continuous and dispersed phase streams at the nozzle and are therefore unsuited to parallelisation. Therefore parallelisation efforts have focussed on step devices in which droplet formation is not dependent on precise input flows.

In this vein, a design modification to the microchannel device was recently proposed alongside claims that it increased the robustness of emulsification due to reliance on a quasi static instability at droplet breakoff, rather than a dynamic one [98]. The new design feature introduced was a gradual widening of the microchannel until meeting the step, such that the droplet grows spherically in the deep channel rather than pancaking in a shallow terraced region. The other innovation was a marketing one, this device was given a memorable name for the resemblance of the dropmaking channels to many tiny legs: the millipede.

The millipede design has been shown capable of forming monodisperse emulsions in hundreds of nozzles simultaneously at diameters down to $20 \mu \mathrm{m}$ [98, 99]. While impressive, this does not cover the full range of droplet sizes achievable using previous devices, in particular the capability to produce droplets in the colloidal size range.

That is therefore the aim of this work: to bring the robustness and low polydispersity of the millipede design to the production of droplets of $1 \mu \mathrm{m}$ in diameter using a glass ${ }^{4}$ device. For utility in colloidal self assembly studies, a (semi-arbitrary) upper limit of $5 \%$ polydispersity is imposed as with high polydispersity, behaviour deviates significantly from that of models of

\footnotetext{
${ }^{3}$ For a review, see Reference 92.

${ }^{4}$ Glass devices involve more effort to fabricate than those from the typical prototype material PDMS. However they have the advantages of more well defined nano-dimensions and much broader solvent resistance.
} 


\begin{tabular}{|c|c|c|c|c|}
\hline $\mathbf{D} \mathbf{( n m})$ & $\mathbf{V}(\mathbf{L})$ & $\mathbf{1} \mathbf{~} \mathbf{~ L ~ a t ~} \mathbf{1} \mathbf{~ k H z}$ & $\mathbf{1} \mathbf{~} \mathbf{m L}$ at $\mathbf{1} \mathbf{~ M H z}$ & Naming \\
\hline 500 & $7 \times 10^{-17}$ & 484 years & 6 months & \multirow{2}{*}{ Attolitre } \\
\cline { 1 - 4 } 1,000 & $5 \times 10^{-16}$ & 61 years & 3 weeks & \\
\hline 3,000 & $1 \times 10^{-14}$ & 27 months & 20 hours & \multirow{2}{*}{ Femtolitre } \\
\hline 10,000 & $5 \times 10^{-13}$ & 3 weeks & 32 minutes & \\
\hline
\end{tabular}

Table 3.1: Illustrative comparison of droplet diameters, volumes and production times. The third and fourth columns give the approximate time it would take to produce $1 \mathrm{~mL}$ of dispersed droplets in a device operating at 1 thousand and 1 million droplets per second, respectively. The former is in the range of a single dropmaking nozzle [88] and the latter represents 1000 such nozzles in parallel. The final column is included as an aid to the colloid scientist used to quoting particle diameters who wishes to consult the microfluidics literature, which is often concerned with droplet volumes [97].

monodisperse particles and will crystallise very slowly or not at all [17, 100]. Another important requirement is sufficient throughput for desired applications. For fundamental studies, a few million dispersed droplets can be sufficient to study, for example, colloidal crystals [95] or glasses [101] and these can readily be made using a single dropmaker. However, for assembly of materials, throughput should approach the gram scale. Table 3.1 illustrates the need for parallelisation if such scales are to be achieved.

The chapter is organised as follows: first a discussion of the relevant theory is presented followed by an account of the device demonstration using a common test system: hexadecane droplets in water. Thereafter, attempts to make materials in the device using photocurable polymer and dispersed nanoparticles are discussed. Finally, suggestions for future work are presented.

\subsection{Theory}

\subsubsection{Droplet Formation}

Pushing a fluid through a small nozzle into a chamber containing a second, immiscible, fluid is not enough to guarantee the formation of droplets. Why should not a single drop of ever increasing size be formed as fluid is added? Under some conditions this may be exactly what does happen [102]. There are two main mechanisms of droplet formation at a microfluidic step junction in the absence of continuous phase flow: the spontaneous dripping and the induced jetting. 

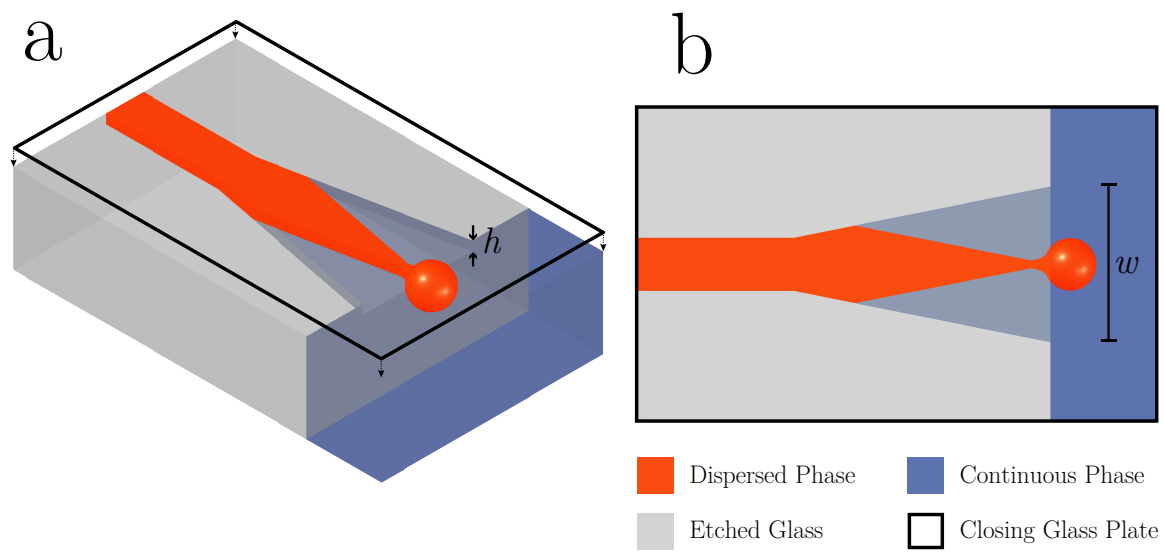

Figure 3.1: Schematic representation of a single nozzle at the moment of droplet release (just before the shrinking neck of the dispersed phase has reached breaking point). The dimensions are proportional to those of the devices used in this work, with an opening angle of $11.3^{\circ}$, $h=200 \mathrm{~nm}, w=3 \mu \mathrm{m}$ and a droplet diameter of $1 \mu \mathrm{m}$. The bottom surface of the closing glass plate is shown slightly raised for clarity.

a) Isometric projection. b) Top view.

Jetting occurs when the dispersed phase emerges from the nozzle at high velocity (relative to the continuous phase), forming a fluid column which disintegrates into droplets as a result of the Rayleigh-Plateau instability [103]. This yields many droplets of approximately the same size as governed by the geometry of the collapsing liquid column, but in general does not match the monodispersity of the droplets formed by dripping, which takes place at lower flow rates.

In the dripping mode, the dispersed phase emerges more slowly, forming a spherical bulb where it emerges into the deep channel (Figure 3.1). This bulb has much lower curvature than that of the interface which is confined by the shallow channel, so there is a drop in Laplace pressure at the step. This drives the growth of the emerging bulb, further increasing the pressure drop and increasing the flow rate over the step. When the flow rate out of the step exceeds that coming in from behind, the tongue shrinks in from the walls and a neck is formed. This neck is unstable and also breaks in a Rayleigh-Plateau manner [104].

Whether dripping or jetting takes place is determined by the dimensionless 
capillary number,

$$
C a=\frac{v \eta}{\gamma}=\frac{Q \eta}{A \gamma},
$$

which indicates the relative influence of viscous and surface forces. Equation 3.1 contains the conventional definition containing the characteristic velocity $(v)$, the viscosity $(\eta)$ and surface tension of the interface $(\gamma)$ as well as the adapted form concerning the volumetric flow rate $(Q)$ across an area, $A$. It was shown in Reference 104 that there is a critical capillary number above which jetting takes place:

$$
C a_{\text {crit }}=\frac{\pi}{128}[2 \cos (\pi-\alpha)-1] .
$$

Here, $\alpha$ is the contact angle of the dispersed phase on the channel wall. This puts a constraint on the conditions required for dripping: the contact angle must be greater than $2 \pi / 3\left(120^{\circ}\right)$. If this condition is not met, the only option is jetting, whatever the capillary number. For practical purposes, this means that the dispersed phase should wet the channel walls as poorly as possible and conversely the continuous phase should have high affinity with the channel walls.

\subsubsection{Droplet Size}

In the same work, it was shown that the droplet diameter varies as

$$
d \sim \frac{h}{\cos (\pi-\alpha)},
$$

with $h$ the channel height in the nozzle [104]. The approximation is that the pressure drop between neck and bulb is low and constant. This was found to agree with experimental and simulation data after scaling with the viscosity ratio.

\subsection{Methods}

\subsubsection{The Nanopede}

The design of the device to produce $1 \mu \mathrm{m}$ droplets is depicted in Figures 3.1 and 3.2. The device was given the working title of 'nanopede' in homage to the millipede design and the smallest channel dimension being $200 \mathrm{~nm}$. The nanopede was fabricated at the University of Twente. Briefly, the design was 
etched into glass using lithography/wet-etching and deep reactive ion etching. This was bonded to an unpatterned glass wafer to close the channels.

The design contains 5000 nozzles divided equally across 5 rows. To yield droplets of around $1 \mu \mathrm{m}$, the height of the channel was $200 \mathrm{~nm}$, the opening width was $3 \mu \mathrm{m}$ and the opening angle was $11.3^{\circ}[104,105]$.

\subsubsection{General emulsification procedure}

All liquids were filtered before use in the nanopede using $0.1 \mu \mathrm{m} \mathrm{PVDF}$ and $0.2 \mu \mathrm{m}$ P TFE (both Millipore) syringe filters for polar and non polar components respectively. The device was prepared by pushing isopropanol (IPA), $1 \mathrm{M}$ $\mathrm{NaOH}$, and isopropanol sequentially backwards (starting at exit) through it, each until the solvent front was visible emerging from both inlets. In this work, the continuous phase ( $\mathrm{CP}$ ) was generally aqueous surfactant solution and the dispersed phase (DP) a hydrophobic solution or dispersion. Liquids were dispensed from $1000 \mu \mathrm{L}$ glass syringes (ILS) mounted on a syringe pump (Cetoni Nemesys 290N) through PTFE tubing (inner diameter $0.8 \mathrm{~mm}$, Bola). The tubing interfaced with the chip via a Micronit $15 \times 30 \mathrm{~mm}$ sideconnect holder using FKKM (perfluoroelastomer, Micronit) ferrules. In the exceptional case of high NA imaging inside the chip, the custom holder shown in Figure 3.2 c was used instead.

The DP was pumped to the top inlet and the $\mathrm{CP}$ to the bottom, as depicted in Figure $3.2 \mathrm{a}$, with a flow rate ratio of $\mathrm{CP} / \mathrm{DP} \approx 50$, e.g. $250 / 5 \mathrm{LL} \mathrm{h}^{-1}$. The outlet used the same connection system as the inlet and the emulsion was collected in a vessel containing the $\mathrm{CP}$.

\subsubsection{Hexadecane Droplets}

The CP was aqueous SDS solution (12 mm Sodium dodecyl sulfate, Sigma Aldrich) and the DP was hexadecane (synthesis grade, Merck). For confocal imaging, the DP contained $1.2 \mathrm{~mm}$ pyrromethene $56_{7}$ dye (P M 567 , Exciton, measured excitation and emission wavelength maxima in hexadecane: $\lambda_{\mathrm{ex}}=$ $\left.520 \mathrm{~nm}, \lambda_{\mathrm{em}}=543 \mathrm{~nm}\right)$. In a separate run under the same conditions, undyed droplets were made for measurement with static light scattering.

The emulsion was collected with $\mathrm{CP}$ and $\mathrm{DP}$ flow rates of 40 and $5 \mu \mathrm{L} \mathrm{h}^{-1}$ respectively for $18 \mathrm{~h}$ in a $4 \mathrm{~mL}$ empty glass vial and left undisturbed for a few days, during which time the droplets creamed. A portion of the resulting dense emulsion layer was drawn into a $0.05 \times 1 \mathrm{~mm}$ rectangular glass capillary and both ends were sealed with UV-curing glue (N O A68, Norland). The capillary was mounted on a standard microscopy slide placed vertically, resulting in creaming of the droplets to one end of the capillary. 

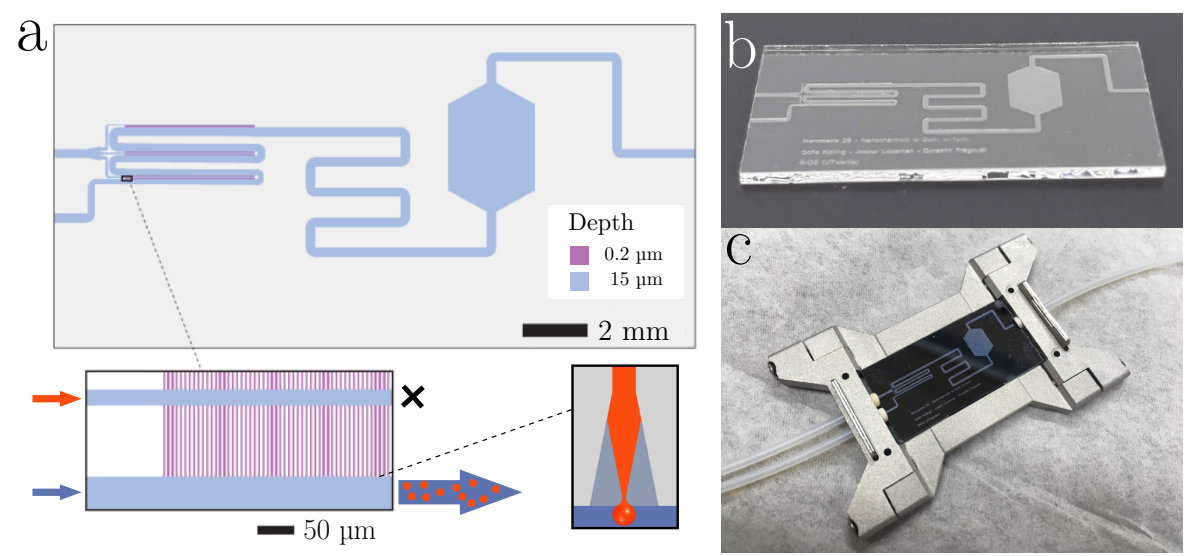

Figure 3.2: The nanopede device. a) The overall design (top) shows the dispersed phase inlet at top left which forks into three channels to distribute the dispersed phase to the 5000 nanochannels. The liquid is pushed through these channels (shown in magenta) and emerges as droplets in the low resistance emulsion channel (connected to the continuous phase inlet shown on lower left). The emulsion passes into the wide observation chamber before exiting the chip on the right hand side. At the bottom, two increasingly zoomed in views are shown. On the left, the arrangement of the nanochannels is shown. Here, the upper orange arrow indicates the dispersed phase (DP) inlet, the lower blue arrow indicates continuous phase $(\mathrm{CP})$ inlet and the ' $X$ ' indicates that the central D P channel leads to a dead-end. On the right, a single nozzle is shown, to place Figure 3.1 in the context of this schematic. b) A photograph of the $15 \times 30 \mathrm{~mm}$ nanopede device. c) Photograph of the custom holder used for high N A microscopy inside the chip. Here a silicon based chip is shown so the features are visible from above, but all results in this chapter were obtained using fully glass chips. 
After several days, images of the droplets were obtained using a confocal microscope (Leica SP 8 with $100 \times / 1.4$ STED oil immersion lens). Ensemble images of diffraction from the crystal were captured using a Nikon Z6 consumer digital camera mounted on a Leica DMIL LED inverted microscope equipped with a $5 \times / 0.12$ air lens.

\section{Imaging Droplet Formation}

To image the droplet formation process at maximal resolution, a high NA objective lens was used (Leica 93x/1.30 s T E D glycerol immersion lens mounted on a Leica D M 6 oо B inverted microscope). This lens has a working distance of $300 \mu \mathrm{m}$ based on a $170 \mu \mathrm{m}$ cover glass, making it unable to focus on the droplet junction in the standard chip design (with a $500 \mu \mathrm{m}$ glass lid). Therefore a chip with a thinner glass lid of thickness $285 \mu \mathrm{m}$ was made, in which imaging was possible.

The brightfield transmission images in Figure 3.7 were captured using a high speed camera (Phantom Miro ex 4 , Vision Research) with framerates of $4000 \mathrm{FPS}$ and 30,000 FPS (exposures $240 \mu \mathrm{s}$ and $30 \mu \mathrm{s}$ respectively). The latter framerate was the highest possible, as limited by the intensity of the illumination light (Leica 100W lamp, condenser N A 0.55). The confocal image in Figure 3.7 was captured at the same junction using the Leica SP 8 attached to the same inverted microscope. The scanning speed was $4 \mathrm{kHz}$ corresponding to a line scan time of $250 \mu \mathrm{s}$ and a framerate of $63 \mathrm{FPS}(256 \times 128 \mathrm{px})$.

\section{Static Light Scattering (SLS) for droplet size distribution measurement}

A dilute suspension (volume fraction, $\phi \sim 10^{-4}$ ) of the droplets in deionised water was prepared and characterised in a custom built SLS apparatus [106]. The scattering intensity of a HeNe laser $(632 \mathrm{~nm}, 10 \mathrm{~mW})$ in the emulsion was measured at angles from $14^{\circ}$ to $135^{\circ}$ relative to the transmitted beam using a photomultiplier tube.

To determine the droplet size distribution, an analytical calculation of the scattering intensity was required. The scattering intensity as a function of angle was calculated using miepython [107, 108], which provides a convenient method for Mie scattering calculations on single spheres. The scattering of a polydisperse size distribution was assembled by summing the scattering intensities of several sphere sizes with a Gaussian weighting around the mean diameter $^{5}$. A least squares optimisation was then carried out to fit this model to the log of the measured intensity distribution, with the mean diameter and

\footnotetext{
${ }^{5}$ This method was tested and found to be in exact agreement with an alternative software: Miescat (v4) by Arnout Imhof, which is based on the algorithm in Reference 109.
} 
polydispersity as fit parameters. It was known that larger droplets were present in the distribution, which contribute most strongly to multiple scattering at low angle (low K) and decrease the depth of the first minimum [110]. Therefore the first minimum and maximum were excluded when fitting the scattering data, in order to characterise only the main droplet distribution. The data and fit are plotted against $K=4 \pi n_{c p} \sin (\theta / 2) / \lambda$ in Figure $3.4 \mathrm{~d}$, with $n_{c p}=1.333$ the refractive index of water and $\lambda$ the vacuum wavelength of the laser. The refractive index of hexadecane used for the scattering calculations was $n_{D}^{20}=$ 1.4345 [111].

\subsubsection{Polymer Particles}

Under safe lighting (all light sources filtered: OD > 7 at $365 \mathrm{~nm}$ ), an emulsion was created using the nanopede. The D P was 10\% E TP TA (trimethylolpropane ethoxylate triacrylate, Sigma Aldrich) in octanol with 0.5 wt.\% photoinitiator Irgacure 2100 and the $\mathrm{CP}$ was 1 wt.\% Pluronic $\mathrm{F} 108$ in deionized water with respective flow rates of $5 \mathrm{LL} \mathrm{h}^{-1}$ and $100 \mu \mathrm{L} \mathrm{h}^{-1}$. The output tubing was directed into a vial containing F108 solution inside a light sealed box continuously illuminated with $365 \mathrm{~nm}$ light (Thorlabs M $365_{5} \mathrm{~L} \mathrm{P} 1,>1150 \mathrm{~mW}$ LED). After collecting for 1 hour, the emulsion was left for a further 30 minutes to polymerise before being retrieved and the resulting particles washed into pure deionised water (by $500 \mathrm{~g}$ centrifugation and supernatant replacement 3 times). $5 \mu \mathrm{L}$ of this washed dispersion was dried on a silicon wafer at room temperature and imaged in SEM (XL3O SFEG scanning electron microscope, FEI), shown in Figure 3.6.

\subsubsection{Supraparticles}

\section{Volatile dispersed solvent: cyclohexane}

The DP was $11 \mathrm{~nm}$ spherical cobalt iron oxide particles [112, 113] dispersed in cyclohexane at a concentration of $4.5 \mathrm{mg} \mathrm{mL}^{-1}$ and cyclohexane saturated $38 \mathrm{mM}$ aqueous SDS solution was the $\mathrm{CP}$. The $\mathrm{CP}$ and DP were pumped through the nanopede at flow rates of 75 and $15 \mu \mathrm{L} \mathrm{h}^{-1}$ respectively and the resulting creaming emulsion was collected as described in Appendix 3.A for 1 hour. At this time, blockages had developed in the oil inlet channels, preventing some of the nanochannels from producing droplets.

The collected emulsion was then transferred to a $1 \mathrm{~mL}$ glass vial containing $0.5 \mathrm{~mL}$ of the same SDS solution. The vial was capped with P TFE tape with a $0.8 \mathrm{~mm}$ hole in the centre and the cyclohexane allowed to evaporate overnight with the vial on a shaker table at $250 \mathrm{R} \mathrm{PM}$. The brown sedimenting dispersion 
was washed 3 times with deionised water by repeated centrifugation at $500 \mathrm{~g}$ and redispersion. The resulting supraparticles were imaged in SEM (Figure $3.8 \mathrm{~b}$ ) and TEM (Tecnai 20 transmission electron microscope, FEI, Figure 3.8c).

To characterise the cyclohexane droplet distribution, the procedure above was repeated but with the nanoparticle dispersion replaced with cyclohexane containing the fluorescent dye P M 567 . A $0.05 \times 1 \mathrm{~mm}$ glass capillary was filled directly from the collection syringe and after creaming, the confocal image in Figure 3.8 a was obtained.

\section{Non-volatile dispersed solvent: hexadecane}

In this case the dispersed phase was a dispersion of the same cobalt iron oxide nanoparticles as detailed above but in hexadecane at the same volume fraction. The continuous phase was $12 \mathrm{~mm}$ aqueous sDs solution. The emulsion was collected for 1 hour as described in Appendix 3. A with flow rates $250 / 5 \mathrm{LL} \mathrm{h}^{-1}$. This was then poured into a vial containing $10 \mathrm{~mL}$ of either ethanol or acetone. The resulting particles were then washed and concentrated in deionised water using centrifugation $(3 \times 500 \mathrm{~g} / 1 \mathrm{~h})$ and redispersion with a final volume of approximately $300 \mu \mathrm{L}$. The particles were imaged with SEM (Figure 3.9).

\subsubsection{Operational Notes}

\section{On flow rates quoted in this chapter}

Two factors contributed to the (especially D P) flow rate at the droplet junctions likely not matching that input to the syringe pump: leaking at inlets and defective nozzles. The first of these was sometimes catastrophic, resulting in no droplets, but often leakage was noted only after an emulsification run when the holder was being dismantled. In the latter event, one or both of the flow rates was lower than intended. In many cases, only a few defective nozzles (either blocked or producing large droplets, see Figure $3.4 \mathrm{~b}$ ) were observed and would likely have negligible effect on the flow through the other nozzles. However, in the thin chip used for confocal imaging, only one working nozzle was found (Figure 3.7), with many blocked and some sections allowing the DP to flow freely into the emulsion channel. Leakage may have been due to a bonding problem and blockage due to glass shards introduced during fabrication. Therefore, in the case of the prototype devices discussed here, the flow rates are comparable only within a single emulsification run and cannot be quantitatively related to actual flow rates through individual nozzles. 


\section{Starting Emulsification}

The main challenge at the beginning of a run was to fill the three DP arms which supply the nanochannels. Simply pumping the dispersed phase at the final flow rate $\left(5 \mu \mathrm{Lh}^{-1}\right)$ was not sufficient to fill these channels within a reasonable length of time. No completely general procedure was found to begin emulsification, presumably due to differences in the solvent properties and in the size of the air bubble often introduced when switching the inlets from IPA to the intended DP. By some combination of the following, with observation of the effects of each via the microscope, it was usually possible to fill $2-3$ arms of the DP supply channel. First, pushing the DP at up to $300 \mu \mathrm{Lh}^{-1}$ for a short time to build the required pressure was sometimes sufficient. Also sometimes helpful was sucking on the outlet with an empty syringe to increase the pressure drop across the whole device, and remove dense emulsion resulting from high DP flow rates.

\section{Chip Cleaning}

While the nanochannels make clearing the chip of blockages difficult and tedious, in several cases it could be returned to a working state after severe clogging with nanoparticles. The general procedure is to flow various filtered solvents backwards (so that no debris is forced through the nanochannels) through the chip. The emulsion/CP passage was never blocked in this work and may be easily flushed with large volumes of solvent due to its wide channels. With this done, this path was blocked ${ }^{6}$, forcing the solvent through the DP inlet. Often high pressures were required which were provided by hand with syringes. This sometimes led to leakages at the chip-tubing interfaces, requiring cleaning of the holder and reconnection.

An alternative method was to use a syringe pump to flow a liquid capable of dissolving the blocking material (e.g. $\mathrm{HCl}$ for nanoparticle clogs) through the chip at low rate for a long time (e.g. $30 \mu \mathrm{Lh}^{-1}$ for 3 days) to slowly dislodge blockages. Ultrasonication in acids, bases, alcohols and alkanes was also used to loosen debris without noticeable damage to the chip.

With the return of a blocked chip to working order usually taking more than a week, prevention was always preferable to cure. Therefore, in the case of potentially clogging DPs, the emulsification was closely observed for signs of blockage and stopped and cleaned at the first sign of clogging. This way, it could usually be cleaned with a single flushing step.

\footnotetext{
${ }^{6}$ A plug may be made by filling the hub of a blunt needle with glue which, after curing, is inserted into the tubing which is to be blocked.
} 


\subsection{Results and Discussion}

\subsubsection{Monodisperse $1 \mu \mathrm{m}$ droplets}

\section{Hexadecane in water: an uncomplicated emulsion}

The first evidence of the creation of monodisperse droplets is visible in Figure 3.3. With so many nozzles in close proximity rapidly producing droplets, the density in the emulsion channel can become very high if there is not a rapid flow removing the droplets as they are created. Colloidal spheres with sufficiently uniform size form crystals at high density and this was evidenced in this chip by the brightly coloured reflections resulting from diffraction at the crystalline layers under transverse white light illumination (Figures 3.3 and 3.4).

The higher magnification image in Figure 3.4c shows the crystal planes directly, allowing an estimate of the droplet size to be made. The plane spacing was found to be $1.15 \mu \mathrm{m}$, close to the design aim of $1 \mu \mathrm{m}$ droplets. Also visible when zoomed in is the undesirable presence of droplets far larger than the crystalline ones (Figure $3.4 \mathrm{~b}$ ). These are presumably formed by nozzles which have taken on shapes different to the design at some point in the fabrication process. Such defective nozzles were found to be present at the same locations on a given chip, independent of flow rates or other operational changes.

While imaging the droplets during emulsification is clearly insightful, it provides only instantaneous information. More important to applications of the nanopede is the ensemble size distribution at the end of a run. Therefore the emulsion was collected and characterised using SLS. The data are shown alongside a calculated scattering curve for a Gaussian size distribution with mean diameter $1.12 \mu \mathrm{m}$ and polydispersity $7 \%$. The fit to the data is good, after excluding the multiple scattering region, and the indicated diameter being slightly lower than the observed crystal plane spacing is consistent. While efforts were made to exclude multiple scattering from the large particles, their single scattering would still contribute to the fitted data, increasing the apparent polydispersity. It is thus concluded that that the $7 \%$ value measured here is an overestimate of that of the main distribution.

To take a closer look at the droplets, fluorescent dye was added allowing them to be imaged using confocal microscopy. Thus was the image showing individually resolved droplets in Figure 3.5 a obtained. It may be seen that the emulsion was sufficiently monodisperse to form colloidal crystals. The outsized particles are clearly visible due to the defects they cause in the lattice. Some very small droplets also became visible, possibly due to satellite formation at droplet breakoff in defective nozzles. The clear peaks in the FF T indicate the 

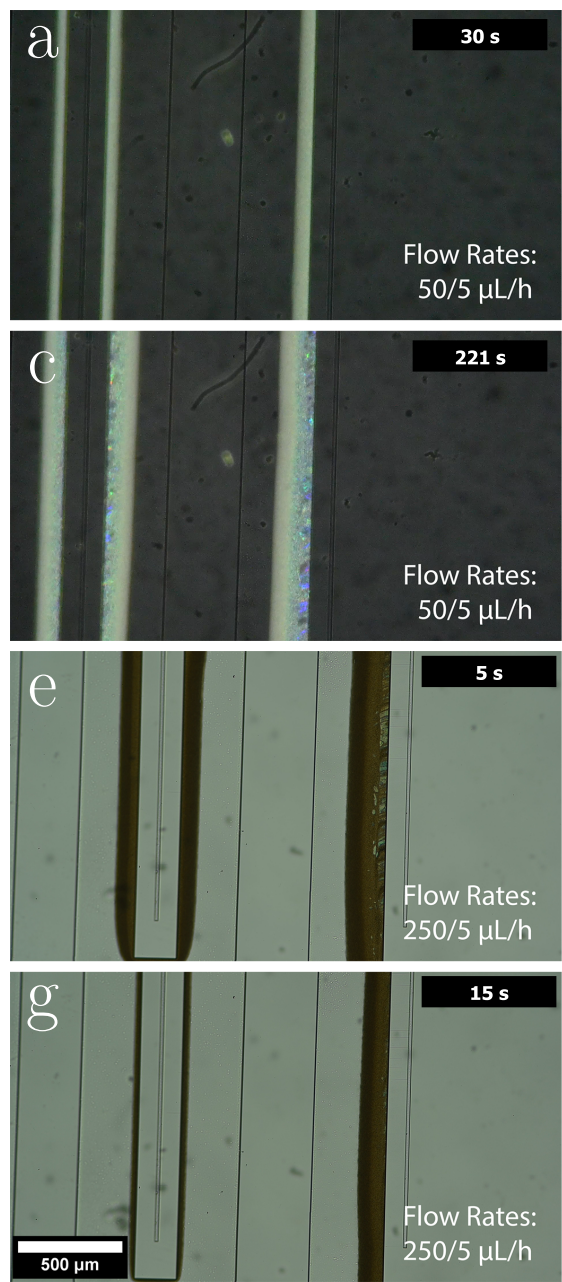
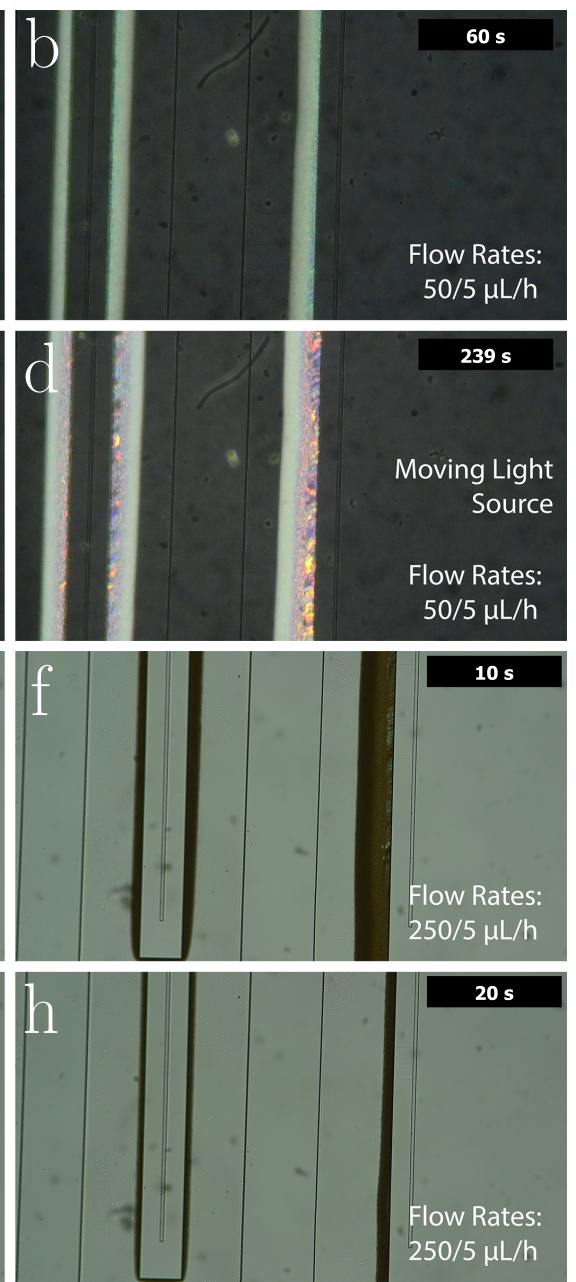

Figure 3.3: Formation of colloidal crystals in the chip when the droplets are not removed rapidly enough from the channel. This was demonstrated by reducing the CP flow rate (before (a)) and then increasing it again (between (d) and (e)). The timestamps indicate the number of seconds since the last change in flow rate and the flow rates are stated as CP/DP. a) 30 seconds after the CP flow rate was reduced from 250 to $50 \mathrm{\mu L} \mathrm{h}^{-1}$. With the lowered flow rate, the stream of droplets (bright in (a-d) and brownish in (e-h) becomes wider. b) Bragg reflections from crystalline droplets started to become visible in the highest concentration channel (right). c) The crystalline regions reached a steady state for these flow rates with progressively larger domains visible as the concentration increases. d) The reflections became more vivid with changing angle of transverse illumination. e-h) When the flow rate was increased back to $250 \mathrm{\mu L} \mathrm{h}^{-1}$, the droplets at the nozzles quickly departed as crystalline rafts and single particles. Production then resumed as before the flow rate was lowered. Images a-d were illuminated from the side, and e-h were illuminated from above, through the condenser lens. 

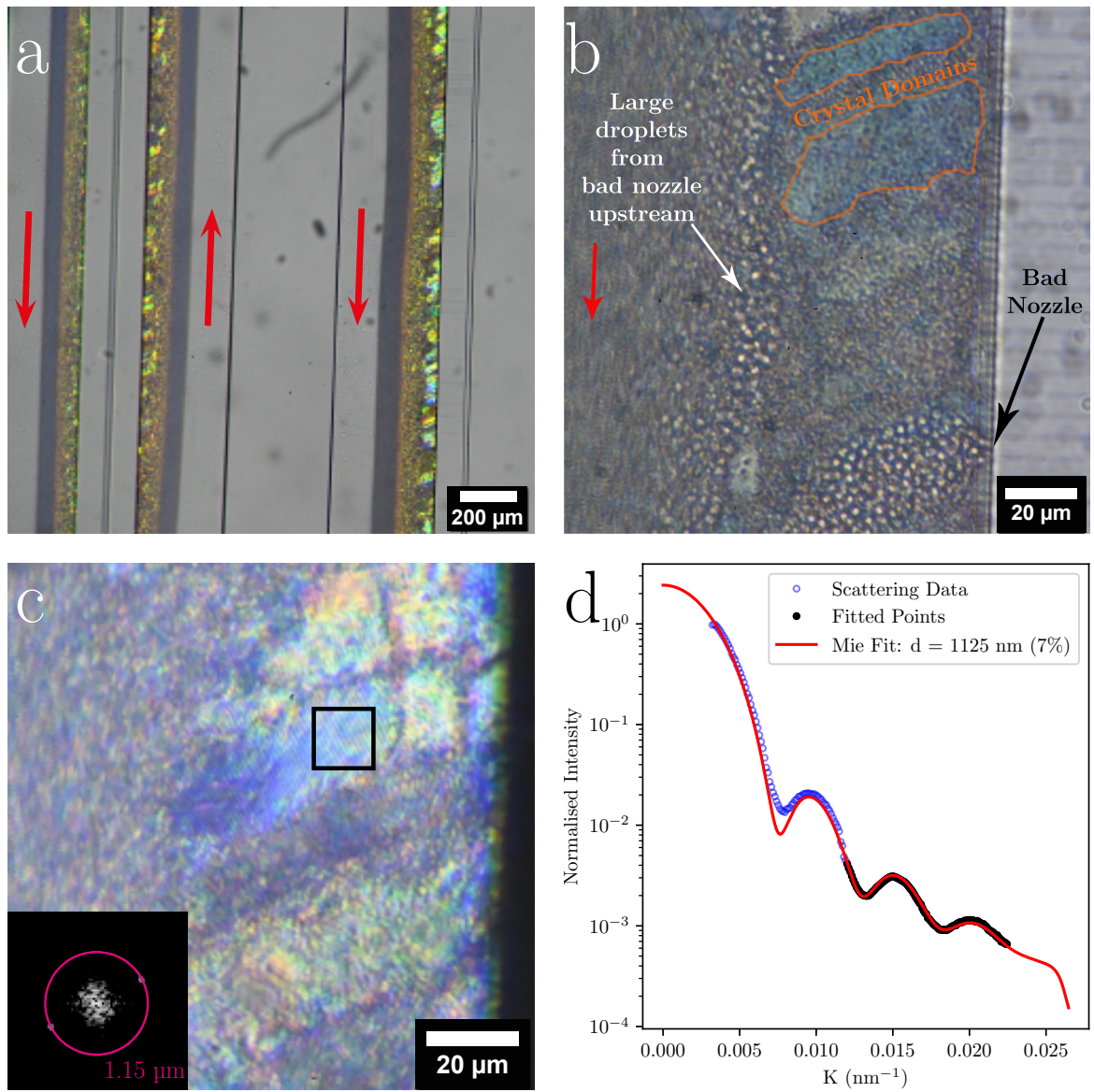

Figure 3.4: Hexadecane droplets. a) Micrograph with both transmission and side illumination showing both the features of the chip and the brightly coloured Bragg reflections. The red arrows indicate the direction of $\mathrm{CP} /$ emulsion flow. b) Higher magnification image with only condenser illumination reveals presence of a defective nozzle producing larger droplets as well as evidence of another one further upstream. c) Transverse illumination of a similar region shows the crystalline domains in colour. Within these, the crystal planes are faintly visible. The inset shows the FFT of the region within the black box, showing peaks at periodicity $1.15 \mu \mathrm{m} /$ cycle. d) SLS data and calculated curve for a Gaussian distribution of spheres with mean $1.12 \mu \mathrm{m}$ and polydispersity $7 \%$. The filled black markers were used for the Mie fit and the empty blue ones were excluded to minimise influence of multiple scattering on the result. 
crystallinity and also the approximate diameter of the droplets, in this case $0.91 \mu \mathrm{m}$. The reason for the dyed droplets being slightly smaller is not clear.

What may be ruled out with some confidence is that the droplets were formed with a diameter of around $1.1 \mu \mathrm{m}$ and then shrunk by $200 \mathrm{~nm}$ by evaporation and/or dissolution while preparing the confocal sample. This shrinkage would reduce the droplet volume by around $40 \%$. Based on a mole fraction solubility on the order of $10^{-10}$ [114], around $60 \mathrm{~m}^{3}$ of water would be required to dissolve this quantity of hexadecane.

The reason for these smaller droplets being formed in the same device is likely related to the contact angle of the oil within the nozzle. It has previously been reported that a larger contact angle leads to smaller droplets [104]. A larger contact angle may have occurred in the case of the dyed droplets due to a surface effect of the dissolved dye or to changes in the surface as the device is used and ages. Further investigation is required to determine the cause of this difference.

Finally, the throughput of the device operating under these conditions is noteworthy. Assuming no leakage, the DP flow rate of $5 \mu \mathrm{Lh}^{-1}$ would yield $1 \mathrm{~mL}$ of dispersed droplets in less than 9 days, corresponding to a production rate of $2.7 \mathrm{MHz}$. In practice, leakage during this $18 \mathrm{~h}$ run was minimal, so the production rate was likely close to this upper limit.

\section{Towards polymer particles and an accurate size distribution}

As the droplet diameter was close to $1 \mu \mathrm{m}$, obtaining an accurate shape of the size distribution from optical images was not possible, with considerable uncertainty in the measurements in Figure 3.5. Therefore an attempt was made to solidify the droplets in order to then measure the size distribution accurately with electron microscopy. Polymerisation of droplets is a well established field with various routes available [115]. For this work, the UV curable monomer ETPTA was chosen such that the droplets would remain liquid until departing the chip and being exposed to UV light.

With this dispersed phase, the new issue of catastrophic polymerisation in the chip was encountered. This was observed to occur when making droplets of pure ETPTA with $1 \%$ initiator, rendering the chip irreversibly blocked. This likely occurred due to the combined effects of a too high initiator concentration and mild UV excitation by the lighting in the laboratory. To prevent this, the monomer was diluted with octanol (additionally allowing the viscosity to be tuned down), the initiator concentration lowered and the fluorescent tube lighting replaced with safe-lighting (see methods in Section 3.3.4). With these modifications, the blocking was prevented and droplets were formed as shown in Figure 3.6 a. 

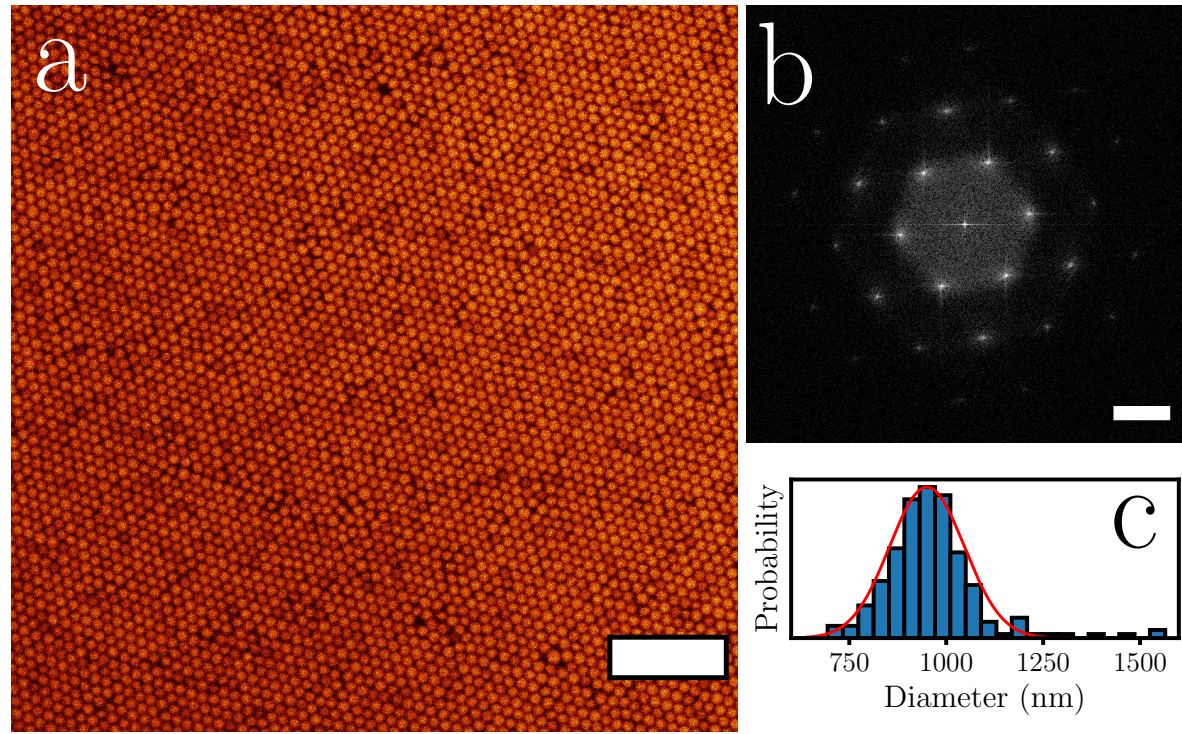

Figure 3.5: Colloidal crystals of hexadecane droplets in water. a) Confocal micrograph of a single layer showing monodisperse droplets in a hexagonally ordered arrangement. b) FFT of a zoomed out image of the same region. The first peaks correspond to a periodicity of $0.91 \mu \mathrm{m}$. The scale bars indicate $10 \mu \mathrm{m}$ and $1 \mu^{-1}$. c) Approximate histogram of droplet diameters based on manual measurement in digital images. The curve shows a Gaussian with mean of $950 \mathrm{~nm}$ and polydispersity $10 \%$ (these calculated from the measured diameters, after disregarding values greater than $1300 \mathrm{~nm}$ ). This approximate method does not give an accurate polydispersity value but does give an indication of the shape of the distribution and the sizes of the larger droplets.

Unfortunately, the polymer particles formed were broken and squashed when observed in SEM (Figure $3.6 \mathrm{~b}$ ). This is presumably due to the polymer particles being deformed either by the washing or drying steps before SEM imaging. Additionally, from the few spherical particles visible in Figure $3.6 \mathrm{~b}$, it may be seen that the diameter of the polymer particles is approximately half that of the original droplets. This shrinkage is expected for polymer particles in the vacuum of the microscope and would not prevent the shape of the size distribution from being measured.

In future work, these problems should be solved by increasing the monomer concentration to increase the strength of the particles. There is still significant 

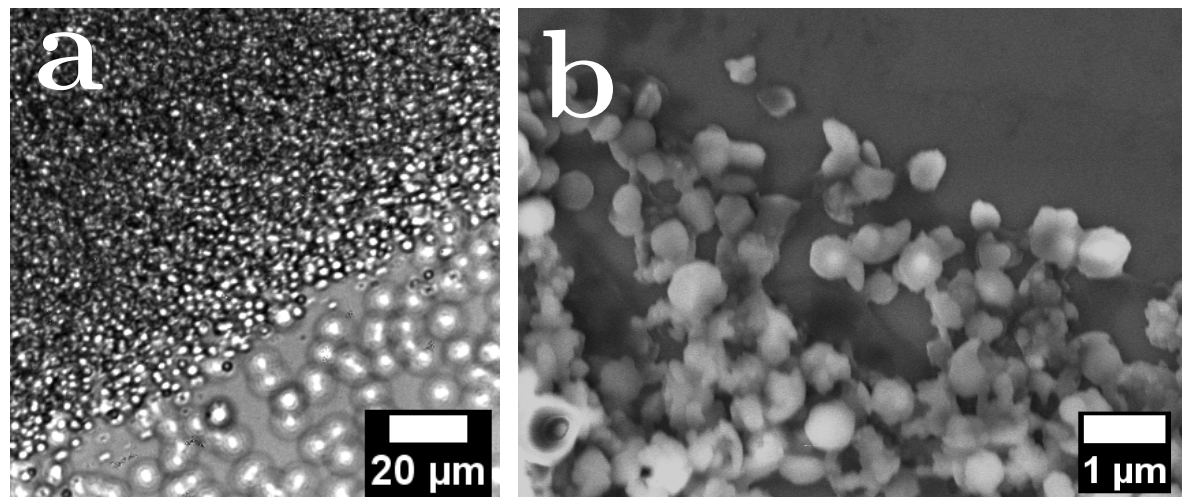

Figure 3.6: a) Octanol/ET P TA droplets of approximately $1 \mu \mathrm{m}$ in the nanopede. b) SEM image of polymerised particles. Most particles did not survive the washing/drying/imaging process. Those that did, have diameter around $500 \mathrm{~nm}$, indicating significant shrinkage in the vacuum of the microscope.

range to do this before the chip begins to block as this was done at the low monomer concentration of $10 \%$.

\subsubsection{Imaging droplet formation}

Droplet formation takes place at very small scale (nozzle height $200 \mathrm{~nm}$ ) and very quickly. Both of these aspects make the imaging of the process challenging, particularly if $3 \mathrm{D}$ information is desired. Figure 3.7 shows images in brightfield transmission which indicate the timescales involved. A droplet was observed to grow and break off in about $10 \mathrm{~ms}$ but the transition to break-off is complete in only $100 \mu \mathrm{s}$.

By counting frames between 18 droplet releases, the average production frequency for the nozzle was found to be $74 \mathrm{~Hz}$ (relative standard deviation $6 \%$ ). Scaling this to a fully working chip with 5000 nozzles yields a production frequency of $370 \mathrm{kHz}$. As only one nozzle was working in this chip, and much of the applied DP pressure presumed to be leaking, this frequency is assumed to be on the low side compared to a fully functional device. Therefore this value was taken to set the lower end of the range of production rates, with the upper end set as the theoretical maximum in Section 3.4.1.

As may be seen from the image at the top right of Figure 3.7, release happens 
too quickly to be probed by currently available confocal microscopes ${ }^{7}$. Even with the fastest scanning on this microscope $(12 \mathrm{kHz}$ bidirectional, $42 \mathrm{\mu s}$ per line), the transition still occurred between line scans.

Confocal microscopy is not known for its time resolution, but for capturing $3 \mathrm{D}$ data. Such information about the droplet formation process would be valuable but this is also obstructed by the poor time resolution. It may be possible to reconstruct one cycle in $3 \mathrm{D}$ by registering frames in videos captured at different heights to points in the cycle. However, at present framerates all this would produce would be a $3 \mathrm{D}$ video of the flattened droplet in the nozzle which is suddenly replaced by the emulsion droplet. The only useful information therein would be the vertical curvature of the front in the nozzle, and even this would be extremely challenging in this device as the vertical height in the nozzle is only $200 \mathrm{~nm}$.

To achieve more informative imaging of the droplet formation process, it will need to be slowed drastically. This may be achievable by increasing the viscosities of both fluids. Also beneficial would be an improvement to the optical situation by bonding the patterned device to a microscopy cover glass. Finally, a device with larger nozzles would make imaging considerably easier, with presumably little mechanistic change.

\subsubsection{Supraparticles}

\section{Droplet Evaporation: volatile dispersed solvent}

A facile route to the synthesis of supraparticles involves using volatile organic compounds such as cyclohexane, with the nanoparticle loaded droplets shrinking due to evaporation of the dispersed solvent. However, the nanopede was found to not function properly with cyclohexane as the dispersed phase. The resulting droplets and supraparticles are shown in Figure 3.8. It was observed that the droplets are both much larger and much more polydisperse than the hexadecane ones.

The reason for this failure, and a solution to it, are explained in Reference 104 wherein it is shown that the similar solvent hexane forms too small a contact angle on glass for stable droplet formation. It is also shown that by modifying the glass surface to make it more hydrophilic, monodisperse hexane droplets were made in a step device, although on a larger scale than that examined here. This requirement for a high contact angle correlates with a requirement for

\footnotetext{
${ }^{7}$ The scanner used here is a fast one capable of achieving framerates of up to 110 fps. The fastest available confocal microscopes can achive framerates $10 \times$ higher, still too slow to image this process.
} 


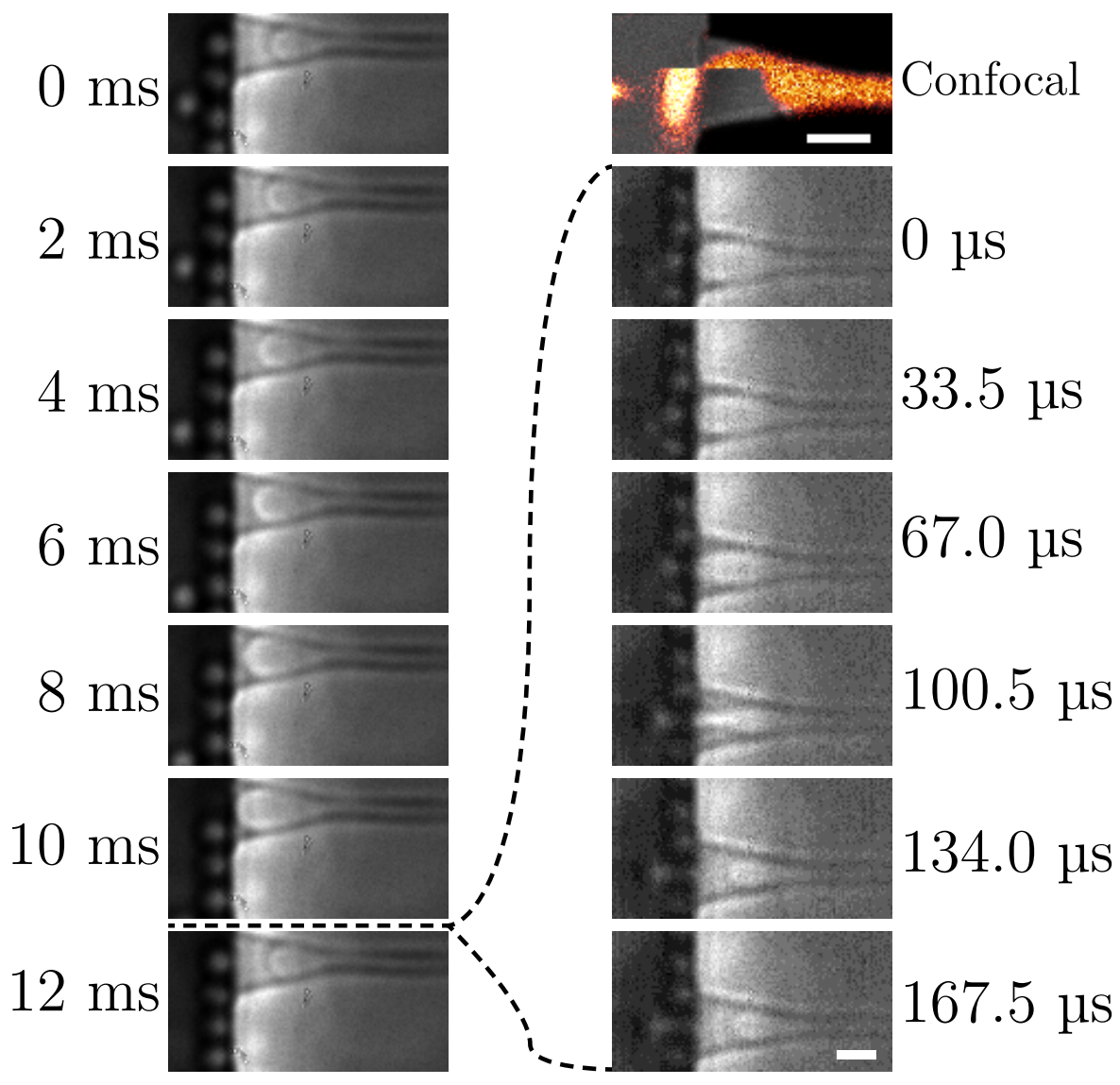

Figure 3.7: Droplet formation in the nanopede viewed in brightfield (timestamped) and confocal microscopy. In all cases, the emulsion/CP flow is from top to bottom. The left column shows one full cycle while the right shows the moment of release at $3 \times 10^{4}$ frames per second. Even at this high framerate, the necking of the droplet is only visible in a single frame (at $100.5 \mu \mathrm{s}$ ). The confocal image (reflection in grey, fluorescence in colour) shows the droplet release event occurring between scans of subsequent lines. The confocal image was constructed by horizontal raster scanning from top right to bottom left, with each line taking $250 \mu \mathrm{s}$ (vertical scan speed $267 \mathrm{\mu m} \mathrm{s}^{-1}$ ). From the high speed brightfield images, it was observed that the breakoff event was completed between the frames at 33.5 and $134 \mu$ s. This means that the confocal records the droplet inside the nozzle for one line scan and the droplet completely released from the nozzle in the next line, giving the apparent straight edges in this image. Examination of the droplet moving downwards in the far left of the left hand column indicates an emulsion flow rate of $263 \mu \mathrm{m} \mathrm{s}^{-1}$. Thus the released droplet moved down the channel at almost exactly the vertical scan rate, making it appear very large and stretched in the flow direction. Both scalebars have length $2 \mu \mathrm{m}$. 

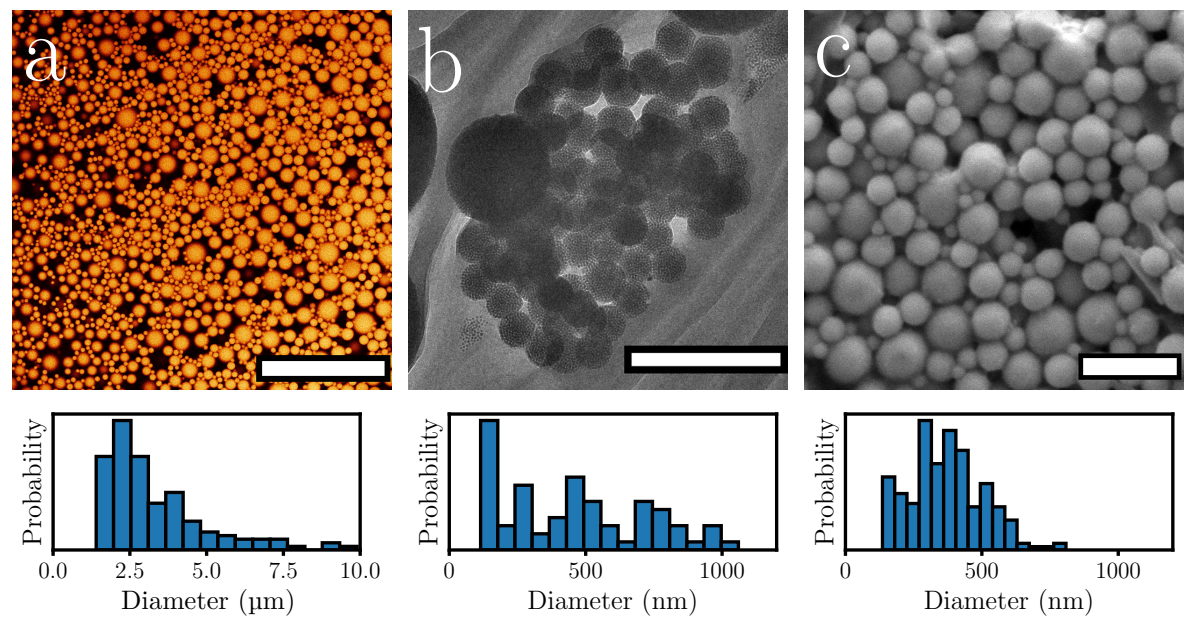

Figure 3.8: Cyclohexane in water emulsions. a) Confocal micrograph of cyclohexane droplets made using the nanopede. b, c) Supraparticles resulting from cyclohexane droplets in TEM (b) and SEM (c) after a further washing step. The scalebars indicate $50 \mu \mathrm{m}, 500 \mathrm{~nm}$ and $1 \mu \mathrm{m}$ respectively and the corresponding diameter distributions are shown below the images.

stronger intermolecular forces and therefore inversely with volatility ${ }^{8}$. Thus the most promising route to making supraparticles by evaporating the solvent is to modify the channel walls to lessen the wetting of the dispersed phase, rather than looking for a different volatile solvent.

\section{Droplet Quenching}

An alternative approach is to disperse the nanoparticles in hexadecane which is known to form monodisperse droplets and remove it by adding a solvent to the continuous phase which increases the DP solubility. Thus the dispersed liquid is further dissolved not by easing the saturation of the $\mathrm{CP}$ by evaporation, but by increasing the saturation concentration. This is not a new idea, and was in fact the method used in work which founded the field of supraparticle assembly [116].

\footnotetext{
${ }^{8}$ High contact angle also correlates with high viscosity, presumably leading to the initial conclusion that the dispersed phase viscosity needed to be higher for stable emulsification in Reference 98.
} 

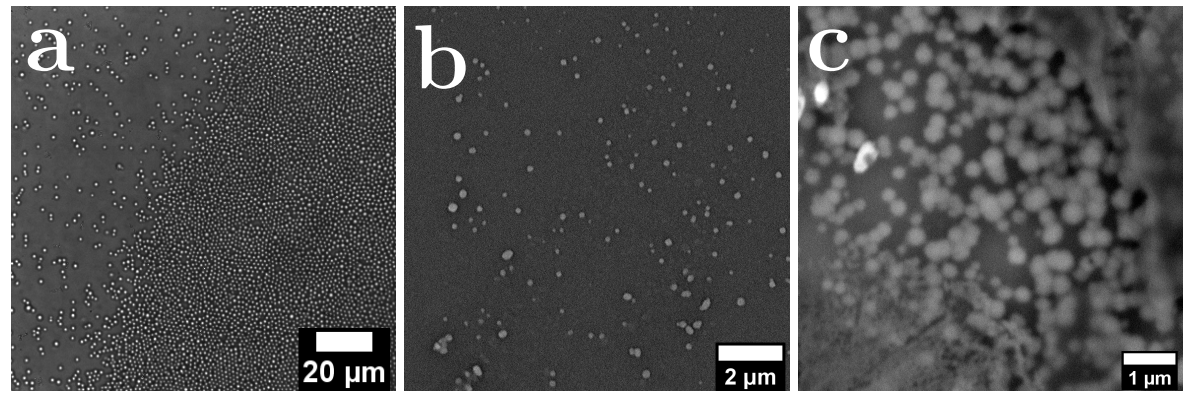

Figure 3.9: Supraparticles formed by dissolution of the dispersed liquid instead of evaporation. a) $1.2 \mu \mathrm{m}$ droplets containing nanoparticles. b) Supraparticles formed by dissolving the hexadecane in ethanol.

c) Supraparticles formed by dissolving the hexadecane in acetone.

Preliminary results obtained by dissolving a hexadecane dispersed liquid in a large quantity of ethanol or acetone are shown in Figure 3.9. It may be seen that the supraparticles formed are more polydisperse than the original droplets and some have merged together and formed ill defined aggregates. It is likely that the injection quenching used here was too violent for the droplets to withstand, with the sudden total miscibility of the dispersed liquid and $\mathrm{CP}$ causing coalescence. A more controlled approach in which the CP composition is slowly altered may yield the low polydispersity supraparticles that would be expected from this emulsion.

\subsection{Conclusion}

The nanopede has been shown to be capable of producing emulsions consisting of droplets with diameter around $1 \mu \mathrm{m}$ and polydispersity below $7 \%$ at rates of up to $5 \mu \mathrm{Lh}^{-1}$ with production frequency between 0.37 and $2.7 \mathrm{MHz}$. This size is lower than that previously achieved in a millipede device and the polydispersity is lower than that observed in microchannel devices forming droplets almost as small as these. With further optimisation of parameters and procedures, this device will become a valuable tool for the preparation of new colloidal systems for use both in fundamental research and the applied synthesis of new materials. 


\subsection{Outlook}

This work shows some very promising results in the synthesis of monodisperse colloidal emulsions, but it is clear that there is more research needed for this method to be fully characterised and reach its full potential. One area for improvement is that of the interface of the chip with the tubing, where leaks often occurred. A way to reduce leakage might be to make the device from thicker glass, thus maximising the surface contact of the ferrules. With the prevention of leaking, the droplet size as a function of capillary number may be properly characterised. Furthermore, contact angle measurements under emulsification conditions will allow greater control and understanding of the process.

For $3 \mathrm{D}$ imaging, the thin glass is of course required but in this case improvements may be made by scaling up and slowing down. Scaling the height of the nozzle channels up to make droplets of 5-10 $\mu \mathrm{m}$ would lift the salient features up away from the spatial limits of confocal imaging and increasing the fluid viscosities would slow down the dynamics, and pull them away from the temporal limits.

Extraction of an accurate size distribution from electron microscopy should also be achievable by increasing the strength of the cured polymer as compared to that in this work. By increasing the monomer concentration at emulsification, the polymer strength may be readily increased.

Formation of monodisperse supraparticles will likely require surface modification of the device surface to increase the contact angle of volatile solvents like cyclohexane. This will present its own challenges in these extremely small channels but nonetheless seems to be the most promising route to this goal.

\section{Acknowledgements}

The work presented in this chapter was carried out in collaboration with researchers at the University of Twente. Corentin Trégouët designed and fabricated the nanopede chips with assistance from Sofie Kölling, Jasper Lozemann and Micronit Microtechnologies B V. Edo de Kruiff designed the holder which allowed high N A microscopy in the nanopede and Mathieu Odijk initiated the project. The images in Figure $3.2 \mathrm{a}$ and $\mathrm{b}$ were made by Corentin Trégouët. In Utrecht, the nanoparticles from which the supraparticles were assembled were synthesised by Ramakrishna Kotni. All are gratefully acknowledged for these contributions and for fruitful discussions. 


\section{A Method to collect a volatile creaming emulsion produced in a microfluidic device}

Figure 3.10 shows collection of creaming droplets of a volatile dispersed phase (DP) while preventing shrinking by evaporation. The plunger is first removed from a large syringe and the nozzle sealed with a rubber septum. It is then mounted vertically and partially filled with the continuous phase ( $\mathrm{CP}$ ) which has been pre saturated with the DP solvent. A shortened Pasteur pipette is inserted with the opening aligned with the back of the syringe nozzle (the sloping at the front of many syringes guides the pipette to the middle, over the opening). An open test tube containing the volatile DP solvent is also inserted to ensure that the air within the syringe is saturated with this solvent, preventing evaporation from the saturated $\mathrm{CP}$ or droplets. A thin needle is pushed through the side of the syringe to allow pressure release and the syringe plunger is replaced. This effectively closed system is allowed to equilibrate for several hours before use.

To collect an emulsion, it is guided into a needle from the tubing and this needle is pushed through the rubber septum such that the end is inside the Pasteur pipette. The droplets then cream inside the pipette forming a concentrated layer at the top which does not evaporate. At the end of collection, the plunger is removed and the pipette retrieved by sealing the top with a finger and lifting it out. The concentrated emulsion may then be pushed and rinsed down into a vial for storage. 


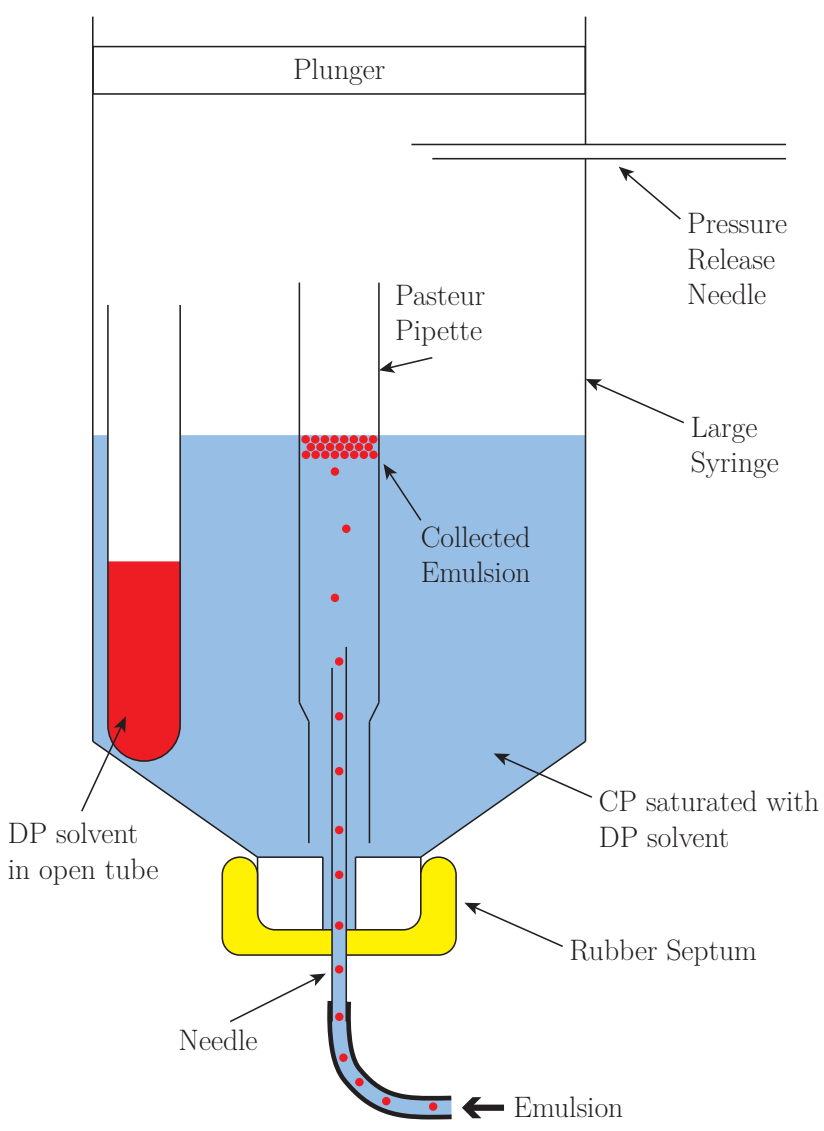

Figure 3.10: Creaming droplet collection method. 



\title{
4 Deep STED microscopy: experimental PSFs and deconvolution
}

\begin{abstract}
Imaging deep inside thick specimens is problematic in all forms of microscopy, and stimulated emission depletion (STED) is no exception. A challenge unique to this technique is engineering the depletion spot shape such that it remains effective at focal planes away from the cover glass. A test sample was developed to characterise the morphing depletion pattern as a function of depth inside the sample and measure the resulting $3 \mathrm{D}$ STED point spread functions (PSFs) at focal depths up to $100 \mu \mathrm{m}$. The sample consisted of three types of silica particle: gold-core (to reflect the incident laser spots), fluorescent-core (to measure P S Fs) and a scaffold of unfunctionalised particles to disperse and immobilise the probe particles in 3 D. A method was developed to maintain the depletion spot shape by adjusting the correction collar of the objective lens for each imaging height. Thus a compact 3 D STED PSF was maintained while imaging deep inside the sample. The measured PSFs were used for experimental depth dependent deconvolution of image volumes of biological and colloidal test samples and the results compared to restorations with theoretical PSFs. The combination of calibration particles may be used for convenient quality control of microscopes for deep STED and confocal imaging.
\end{abstract}




\subsection{Introduction}

All imaging systems from the largest telescopes to the smallest 'eyeball' bacteria $^{1}[117]$ share a common purpose: to obtain spatial information about the world $^{2}$. Unfortunately for bacteria and astronomers alike, the images created by these systems do not exactly represent the form of the world. In fact, they show a version of the scene which is altered in ways which depend on the properties of the imaging system. For example, images projected onto the human retina are upside down, in contrast to the real world objects they represent. This is corrected in post-processing by the brain which uses knowledge of the image formation process to bring the image closer to reality. While this is effortlessly corrected, other aberrations such as blurring due to a cataract in the lens are not.

Even when the imaging system is a high quality device designed and manufactured to be as close to perfect as possible, it is still unable to produce an image without adding its own specific distortions. This is the case of diffraction limited imaging, where the finite size of the light manipulating system necessarily results in diffraction, which ultimately limits the imaging resolution. Such a system creates an image of a point light source as a series of concentric rings in two dimensions: the familiar Airy disk [118].

This chapter is concerned with the optical microscope, one example of a diffraction limited imaging system. The design requirements for a microscope to reach the diffraction limit, as well as where this limit lies, were demonstrated in the $19^{\text {th }}$ century by the optical pioneer Ernst Abbe [119]. The first minimum of the Airy disk in a diffraction limited image is located at a distance of

$$
r_{\min }=0.61 \frac{\lambda}{n \sin \alpha}
$$

from the central maximum (as measured in the object plane). $\lambda$ is the wavelength of light in vacuum, $n$ is the refractive index (RI) of the lens working medium and $\alpha$ is the half-angle of the cone of light acceptable by the objective lens. The denominator in Equation 4.1 is the numerical aperture (NA). For a modern oil immersion objective lens with $\mathrm{NA}=1.4, r_{\min } \approx 220 \mathrm{~nm}$.

The world which is to be imaged is of course a three dimensional place, and the Airy disk is a slice through a $3 \mathrm{D}$ diffraction pattern which is a series of

\footnotetext{
${ }^{1}$ Reference 117 describes a spherical bacterium in which light incident on one side is focussed onto the opposite side, forming an image. Thus the entire organism behaves like a tiny eyeball.

${ }^{2}$ This is a very broad definition, worded to include the electron microscopist and indeed the stencil artist. The focus of this discussion is optical imaging, although many of the concepts are more broadly applicable.
} 
cones with rotational symmetry about the optical axis. This response to a point source is known as the point spread function (PSF) and it contains a great deal of information about the imaging process. The first minimum in intensity along the optic axis is located at a distance of

$$
z_{\min }=2 \frac{\lambda n}{\mathrm{NA}^{2}}
$$

from the central maximum [120]. For the immersion lens mentioned above, $z_{\text {min }} \approx 775 \mathrm{~nm}$, indicating a much lower axial than lateral resolution with the disparity decreasing linearly with increasing NA.

The problem with a PSF which extends as cones far above and below the focal plane is that, when not imaging an isolated point object, blurred light from out of focus objects appears in the focal plane, reducing the contrast of the in-focus objects. The utility of such a microscope for imaging extended objects is limited to thin specimens, in which this out of focus signal is minimal. An ingenious solution to this problem was found in 1957 by Marvin Minsky [121].

The innovation was the insertion of two axial pinholes of radius close to $r_{\text {min }}$ into the optical path at conjugate focal planes in front of the illumination source and the detector. The first of these has the effect of illuminating only one lateral point in the object plane and the second acts to reject light originating from out of focus planes above and below. This means that extended objects can be imaged as if they were series of isolated point sources, with the whole object being imaged by laterally scanning through the illumination point. The rejection of out of focus light additionally allows the confocal microscope to record $3 \mathrm{D}$ images. This is achieved by recording 'stacks' of images at different focal depths in the sample. The PSF contains components of the diffraction patterns of the illumination and detection spots, which in the confocal arrangement both have the form of the same Airy pattern. Therefore the confocal PSF is approximately the square of the widefield one. This does not move the positions of the minima but does have the effect of increasing the intensity of primary features compared to secondary ones. With sharper central maxima, two objects may be closer together before becoming unresolvable. This may be further improved by decreasing the size of the exit pinhole so that it rejects a greater portion of the Airy pattern. This improvement in resolution approaches a factor of $\sqrt{2}$ approaching an infinitely small pinhole. However, decreasing the radius of the pinhole below $r_{\min }$ reduces the signal to noise ratio $(\mathrm{SNR})$ by rejecting potentially useful light.

The modern confocal laser scanning microscope (CLSM) operates using much the same principles, except that the point illumination is a laser spot and lateral scanning is done by this spot rather than the sample. The exit pinhole is still present, and generally has an adjustable radius to accommodate different 
imaging requirements. While the above discussion was general to transmission and epi-illumination systems, CLSM is mostly used in the latter mode and usually as a fluorescence microscope. Fluorescence imaging is possible deep in an optically homogeneous medium whereas imaging anything in brightfield requires that the medium refractive index not be homogeneous, resulting in distortions at greater depths. The use of laser illumination also lends itself to fluorescence mode, because the coherence of the laser is not retained in the emission signal, maximising resolution [122].

While confocal imaging has contributed to important advances in the fields of cell biology and condensed matter physics [123], it has limits and various efforts are ongoing to circumvent them. One of these is stimulated emission depletion (STED) in which fluorophores peripheral to the focal spot are darkened using a second laser beam having a focal pattern containing a central minimum [124126]. In the ideal case, the depletion spot is a diffraction pattern with zero intensity at its centre, meaning that the resolution is not diffraction limited but may be arbitrarily increased by increasing the depletion beam intensity [127]. However, as in the confocal case, the gain in resolution is paid for by a reduction in SNR and unlike a pinhole, the high depletion laser intensity can significantly damage the object being imaged.

One successful way to achieve such a depletion beam utilises a spatially variant phase plate to cause destructive interference in some parts of the depletion spot. For example, an annular half wave plate inducing a phase shift in a central spot only can yield a spot with intense axial lobes and a weaker lateral ring around a central minimum [128]. This pattern is the result of destructive interference of the PSF from the outer phase ring and the larger PSF from the inner spot, which only illuminates the central portion of the lens back aperture $[129,130]$. This configuration is encountered frequently in this Chapter (see e.g. Figure 4.8) and is referred to as ' 3 D STED'.

Alternative to this is a vortex phase plate, which results in a cylindrical depletion spot with a dark hole through its centre, becoming the characteristic STED 'doughnut' when viewed in cross section $[130,131]$. This is referred to as ' $2 \mathrm{D}$ STED', and it is able to achieve lateral resolutions of a few tens of $\mathrm{nm}$ [132-134]. Combinations of different proportions of the $2 \mathrm{D}$ and $3 \mathrm{D}$ depletion shapes may be used to meet specific imaging requirements.

Despite all these developments, imaging deep inside an extended specimen still presents significant challenges. Many of these stem from inhomogeneities in the optical path in front of the objective lens. Refractive index variations at the front and back of the cover glass introduce depth dependent spherical aberrations which distort images captured deep in the sample [135]. In the case of STED imaging, the effect is compounded as the depletion light is also subject to aberrations. This can cause expansion of the central minimum, reducing 
resolution, or shifting of the pattern such that useful signal is depleted [136138]. Efforts have been made to reverse these aberrations by modifying the optical path in two principal ways [139]. The first of these is to adjust the correction collar ( CC) of the objective lens at each imaging height [140] and the second involves the use of adaptive optics such as spatial light modulators and deformable mirrors [141]. The former approach is used in this work due to its ease of implementation in already commercially available microscopes.

The goal of this work was twofold. First, it was desired to develop a test sample allowing measurement of $3 \mathrm{D}$ STED point spread functions far from the cover glass, at heights of tens of $\mu \mathrm{m}$. This would represent comprehensive characterisation of the imaging ability of one of the first commercially available systems capable of deep 3 D S TED microscopy. The second aim was to push the resolution limit of $3 \mathrm{D}$ STED microscopy in thick specimens by deconvolution with the measured depth variant PSFs.

\subsection{Theory}

\subsubsection{Image formation}

CLSM is an imaging technique which may often be approximated as linear and shift invariant [142]. The former results from the incoherent emission, which means that the image may be described as a superposition of PSFs resulting from a corresponding series of point sources composing the object. The latter means that the PSF is the same for all positions. These two properties allow the image, $i(\boldsymbol{r})$, to be described by a convolution of the ground truth object $(f(\boldsymbol{r})$, the distribution of fluorophores in space) with the PSF, $h(\boldsymbol{r})$ :

$$
i(\boldsymbol{r})=f(\boldsymbol{r}) * h(\boldsymbol{r})+n(\boldsymbol{r}),
$$

where $*$ denotes convolution and $n(\boldsymbol{r})$ is noise.

It is also beneficial to consider this process in reciprocal space. Taking the Fourier transform of Equation 4.3 yields:

$$
I(\boldsymbol{k})=F(\boldsymbol{k}) \times H(\boldsymbol{k})+N(\boldsymbol{k}),
$$

using the convolution theorem and linearity. The object $F(\boldsymbol{k})$ is now considered to be composed not of point light sources but of spatial frequency harmonics (which in isolation are sinusoidal gratings). Low frequency harmonics are associated with large features and smooth gradients while high ones correspond to fine detail and sharp edges. It is clear from Equation 4.4 that the extent to which each of these frequencies is reproduced in the image is determined by 
$H(\boldsymbol{k})$. This is the Fourier transform of the PSF, known as the optical transfer function (OTF). The OTF always drops to 0 at a certain spatial frequency, defining the bandwidth of the instrument (black in Figure 4.1). Below this, its specific shape determines how different features of the image are blurred [143].

\subsubsection{Deconvolution}

With an understanding of the imaging process (Equation 4.3), the natural progression is to attempt to reverse it and recover the true object. The way do do this would seem to be to divide through Equation 4.4 by the OTF, $H(\boldsymbol{k})$, obtainable from a measured or computed PSF. This simplest approach is known as inverse filtering, and it generally does not yield useful results. The problem lies in the fact that noise is present at all frequencies up to the sampling one and when this is divided by the very low values in the OTF (see Figure 4.2), high frequency noise dominates the result, obscuring all useful information [145].

\section{Wiener Filtering}

This noise amplification may be partially brought under control, at the cost of some spatial resolution, by replacing $H(\boldsymbol{k})$ with $H(\boldsymbol{k}) /\left(H(\boldsymbol{k})^{2}+C\right)$, where $C$ is a small constant chosen to only be significant when the OTF has very low values. This is known as Weiner filtering and it is able to recover useful information from distorted images [146]. However, the value of $C$ must be chosen empirically ${ }^{3}$, with the choice affecting the whole restored image in a somewhat arbitrary way [143].

\section{Nonlinear constrained iterative methods}

Currently widely used deconvolution methods have largely departed from linear ones in favour of nonlinear constrained iterative algorithms. These allow extra information to be added to the restoration process, such as the constraint that fluorescence intensity cannot be negative [143]. The goal is to iteratively improve an estimate of the original object, $f^{j}$, using the following general procedure.

1. Choose an initial estimate $f^{0}$. Often the measured image or a blurred version of it are suitable choices.

\footnotetext{
${ }^{3}$ In fact, $C$ is an approximation to a term in the full Wiener filter: $H /\left(H^{2}+F^{2} / N^{2}\right)$, which estimates an object with minimal mean square error compared to the ground truth. The approximation is required because the autocorrelation of the ground truth object $\left(F^{2}\right)$ is not known. See Reference 146.
} 


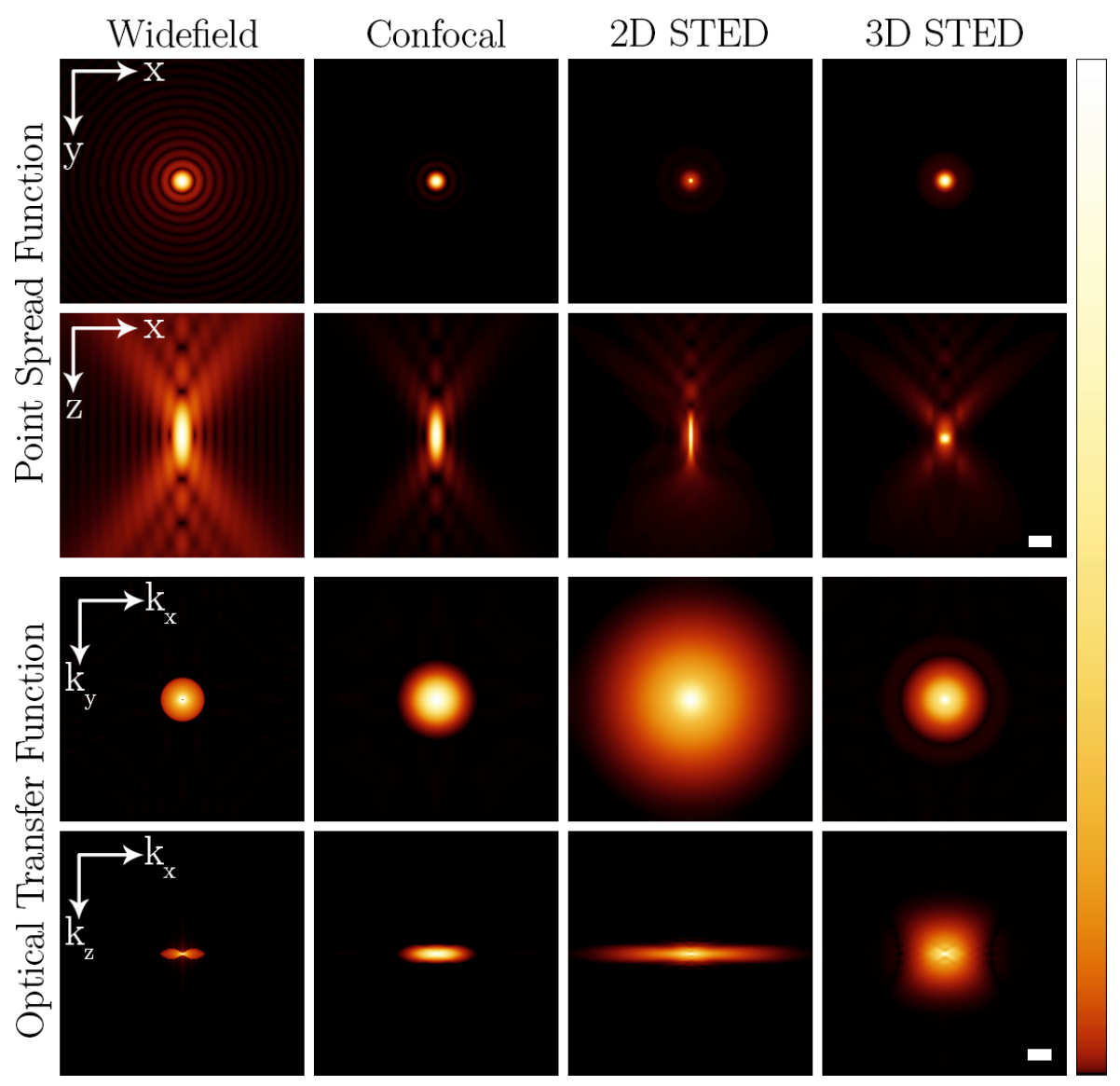

Figure 4.1: Theoretical PSFs and corresponding OTFs for various imaging modes of an oil immersion objective with $\mathrm{NA}=1.4$, imaging in immersion oil. The increase in bandwidth with decreasing PSF size is visible. The scalebars have length $500 \mathrm{~nm}$ and $5 \mu^{-1}$ and the colourbar shows linearly increasing grey values with the same lookup table $(\gamma=0.3)$. The widefield PSF was calculated using the Born and Wolf model [144] and its square used to approximate the confocal PSF. The S TED PSFs were calculated using Huygens software (see methods for details). 

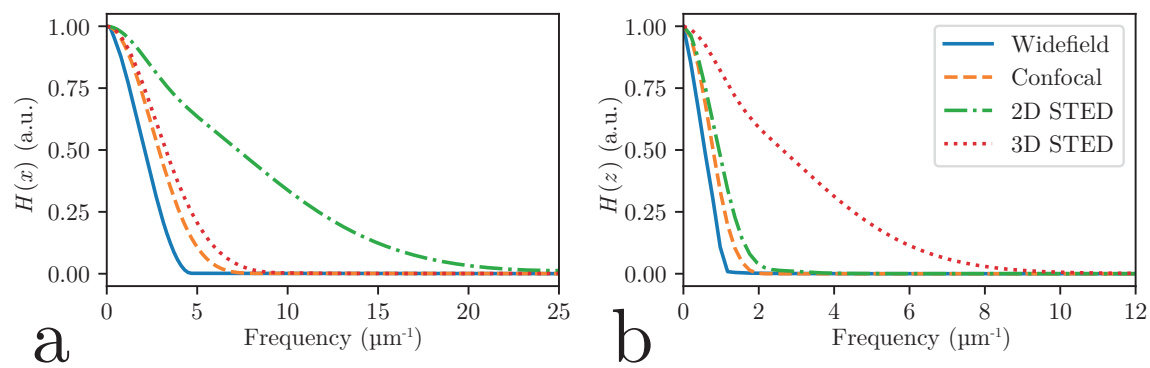

Figure 4.2: Integrated theoretical optical transfer functions in the lateral (a) and axial (b) directions. All are scaled by their maximum value to allow comparison. In both cases, the theoretically achievable gains in resolution with STED are clear.

2. Compute a new estimate, $f^{j+1}$, using $f^{j}, i$ and the PSF, $h$.

3. Apply the constraints to $f^{j+1}$. For example, by setting all the negative pixel values to 0 .

4. Check if some stopping criterion is met and if not, repeat from step 2. A convenient stopping criterion could be when the relative difference between estimates $\left(\frac{f_{j+1}-f_{j}}{f_{j}}\right.$ falls below some value, e.g. $\left.10^{-4}[143]\right)$.

This recipe hides the complexity and diversity of options in computing a new estimate, while ensuring that it is better than the previous one. The accuracy of the estimate can be checked by convolving it with the PSF and comparing it to the measured image. This is the basis of the Jansson/van Cittert method $[147,148]$ in which the next estimate is computed as follows:

$$
f_{j+1}=f_{j}+\epsilon\left[i-\left(f_{j} * h\right)\right] .
$$

The estimate is altered by adding some fraction, $\epsilon$, of the error signal $i-i_{\text {est }}$, which should lead to successively better estimates. This algorithm has achieved considerable success, despite having the drawback that it does not account for noise and this makes the optimal restoration ambiguous [143].

A class of methods which includes a specific noise model is Maximum Likelihood Estimation. Here the problem is considered as a fitting one, with every pixel in $f$ a parameter. A good choice of parameters should have finite probability of measuring the data which were observed, given a specific noise model. Therefore the goal is to find $f$ which maximises the likelihood that the measured image would be produced. Leaving out the details of the derivation, in 
the case of Poisson noise, the estimate is now updated using Equation 4.6 [143]:

$$
f_{j+1}=f_{j} \times \mathcal{F}^{-1}\left\{\mathcal{F}\left\{\frac{i}{f_{j} * h}\right\} \times H^{*}\right\} .
$$

$\mathcal{F}^{(-1)}$ denotes the (inverse) Fourier transform. Multiplication by the complex conjugate of the o TF, $H^{*}$, in the Fourier domain corresponds to cross correlation of the PSF with the relative difference $\left(i /\left(f_{j} * h\right)\right)$. Intuitively, this may be understood by considering a feature missing from the estimate. A peak will appear in the difference map which will become a blurred peak when cross correlated with the PSF. The signal in that region of the estimate will be amplified when multiplied by $f_{j}$. This is the Richardson-Lucy algorithm [149, 150].

\section{Regularisation}

The final component to be discussed here is regularisation. This is a procedure widely used in inverse problems such as deconvolution. The general aim is to push the restoration result towards a reasonable result based on knowledge of the problem, adding this knowledge to improve the conditioning ${ }^{4}$ of the problem. Often this takes the form of penalising high amplitude oscillations in the deconvolved image, hence reducing noise amplification [143]. How much regularisation should be imposed depends on the particular image, with too little allowing spurious features to appear from the noise and too much suppressing real detail.

This discussion is limited to the currently available methods with suitably user friendly implementations for general microscopists. Image deconvolution is an active field of research and is likely to see many improvements in the coming years with advancements in signal processing and through incorporation of novel machine learning techniques [151].

\section{Spatially variant deconvolution}

A foundation of the preceding discussion was the assumption that the microscope is shift invariant. One case in which this assumption becomes invalid is that of imaging volumes with large $z$ dimension in an optically mismatched medium. Here, the PSF far from the objective will be significantly deteriorated compared to that at the opposite end of the volume, rendering the assignment of a single transfer function to the whole volume meaningless. The simplest way to deal with this is to divide the image volume into slabs along $z$ and deconvolve each of these with a different PSF [152, 153]. This allows use of the

${ }^{4}$ 'Conditioning' here refers to the sensitivity of the numerical solution to small changes in the data. The deconvolution problem is generally ill conditioned. 
well developed methods described above if one accepts the inherent coarsening of the restoration. It is this route which is taken in this chapter, but it is noted that research is ongoing into more rigorous methods of spatially variant deconvolution [154] and the results of this chapter may be readily incorporated into these and novel methods appearing in the future.

\section{Obtaining a PSF}

The restoration schemes discussed above require that the PSF of the microscope is somehow known. The two main options for acquiring this are to compute it based on optical theories or measure it using a sub-resolution object ${ }^{5}[155,156]$. Methods to measure the confocal PSF are well established [156] and measurements as a function of depth have been demonstrated using beads on a tilted cover glass [157] and using a single bead translated with an optical tweezer [158]. In STED microscopy, direct acquisition of a PSF is more difficult due to the limited signal from the $\sim 50 \mathrm{~nm}$ beads which are required. Despite commercial availability of fluorescent probes designed for this purpose [159], studies focussed on measurement of STED PSFs have not been reported, let alone a study of the depth dependent behaviour.

\subsection{Methods}

\subsubsection{Sample Preparation}

\section{Particles}

Three different types of silica particle were used, each with a specific role. For measuring the PSF, the fluorescent probes (Figure $4.3 \mathrm{~b}$ ) consisted of a $45 \mathrm{~nm}$ R I T C-labelled core coated with Stöber silica up to a diameter of $505 \mathrm{~nm}$ (polydispersity $(\delta)$ expressed as ratio of the width of the distribution and its mean $<2 \%$ ). To image the depletion laser beam, particles with an $80 \mathrm{~nm}$ gold core were grown to the same diameter as the fluorescent particles as described in Reference 160 were used. Finally, the scaffold particles were made using a standard method based on that described in Reference 25. Specifically, $6.6 \mathrm{~mL}$ of tetraethyl orthosilicate (TEOS, 98\%) was pipetted under the surface of a mixture containing $120 \mathrm{~mL}$ absolute ethanol and $15 \mathrm{~mL}$ ammonium hydroxide solution ('ammonia', $28 \mathrm{w} \% \mathrm{NH}_{3}$ in $\mathrm{H}_{2} \mathrm{O}$ ) under vigorous stirring in a $250 \mathrm{~mL}$ round bottomed flask at $30^{\circ} \mathrm{C}$. The stirring rate was reduced to $300 \mathrm{rpm}$

\footnotetext{
${ }^{5}$ There is a third option, which is to optimise an estimate of the PSF alongside the estimate of the object. This is called blind deconvolution and has its own merits, but is adjacent to the scope of this chapter.
} 
after 10 minutes and the mixture allowed to react for $24 \mathrm{~h}$ before washing the particles three times by centrifugation $(700 \mathrm{~g} / 45$ minutes) and redispersing in ethanol. This yielded the particles shown in Figure $4.3 \mathrm{c}$ with a mean diameter of $307 \mathrm{~nm}(\delta \sim 11 \%)$.

Mixed dispersions of the particles in ethanol were made such that the number ratio of unlabelled host : fluorescent : gold-core particles was 85000 : 99 : 1 (19500 : $99: 1$ by volume). This mixture was chosen for an average separation between fluorescent particles of approximately $\sqrt[3]{85000 / 99} \approx 10$ scaffold particles, or $\sim 3 \mu \mathrm{m}$.

\section{Index Matching Liquid}

For the fluorescent and reflective probes to be suspended in an optically homogeneous medium, the cell must be filled with a liquid with the same refractive index as the scaffold particles. A mixture of glycerol $\left(\mathrm{n}_{\mathrm{D}}^{20}=1.474\right.$ [63] $)$ and 1-butanol (99.8\%, Sigma Aldrich, $\mathrm{n}_{\mathrm{D}}^{20}=1.400$ [161]) was used to measure the refractive index of the scaffold particles ${ }^{6}$. To determine the index matching solvent ratio, a series of mixtures with increasing butanol concentration were made and scaffold particles dispersed in each to a volume fraction of $1.3 \%$. Transmission spectra were measured of these dispersions (HP 8452a spectrophotometer)and the mixture with maximal transmittance at $500 \mathrm{~nm}$ was deemed to have the same refractive index as the particles at this wavelength and temperature $\left(21^{\circ} \mathrm{C}\right)$. This was found to be at $61 \%$ butanol in glycerol (Figure $4.3 \mathrm{e}$ ), corresponding to a refractive index of $\mathrm{n}_{\mathrm{D}}^{21}=1.423$ as measured using an Atago $3 \mathrm{~T}$ Abbe refractometer (Figure $4.3 \mathrm{~d}$ ).

\section{Microscopy Cell}

A schematic showing the cell design is shown in Figure $4.3 \mathrm{a}$. First, $5 \mu \mathrm{L}$ of the mixed particle dispersion $(18 \mathrm{mg} / \mathrm{mL})$ in ethanol was repeatedly $(5-10 \times)$ dropcast onto the same spot on a cover glass (Marienfield Superior \#1.5H), with the ethanol rapidly evaporated at $50{ }^{\circ} \mathrm{C}$ between each drop addition. The resulting residue was uneven with regions more than $100 \mu \mathrm{m}$ high after several steps. This heap of dried particles was incorporated into a channel of height $\sim 200 \mu \mathrm{m}$ suspended beneath a microscope slide, shown in Figure $4.3 \mathrm{a}$, using U V glue and \#1.5 cover glasses as spacers. The channel was filled with $63.0 \mathrm{w} \%$ glycerol/water $\left(n_{D}^{21}=1.4230, V_{D}^{21}=59.7\right)$, chosen to match the refractive index of the scaffold particles, and subsequently closed with UV-curing glue (NOA68,

\footnotetext{
${ }^{6}$ In the notation $\mathrm{n}_{\mathrm{D}}^{x}$, the subscript refers to the wavelength at which the refractive index is being quoted, in this case that of the sodium doublet line at $589 \mathrm{~nm}$. The superscript indicates the temperature in ${ }^{\circ} \mathrm{C}$.
} 
Norland). $61 \%$ butanol was not used in the cell because it was found to be incompatible with the glue (causing cell leakage).

\section{Deconvolution Test Samples}

A spheroid culture of Caco-2 cells was prepared [162] with actin filaments stained using Alexa Fluor 568 Phalloidin. These were mounted in Prolong Diamond (Thermo Fisher) with a curved \#1.5 borosilicate cover glass. The buffer which contained the cells prior to mounting was partially incorporated, reducing the refractive index of the sample medium to a value between that of water and Prolong Diamond $(\sim 1.47)$. The exact sample refractive index is a crucial parameter in deconvolution so this value was measured after cell preparation by measuring the critical angle in a TIR microscope as described in Reference 163. This indicated a sample refractive index: $\mathrm{n}_{561 \mathrm{~nm}}^{25}=1.395$.

The actin filaments were imaged using the same lens and immersion as described above with $568 \mathrm{~nm}$ excitation and detection around $600 \mathrm{~nm}$ with gating from $2-7 \mathrm{~ns}$. Image volumes of dimensions $16.7 \times 16.7 \times 5.1 \mu \mathrm{m}$ (voxel dimensions $80 \times 80 \times 33 \mathrm{~nm}$ ) were captured at $20 \mu \mathrm{m}$ height intervals.

A colloidal test sample was made in a similar fashion to the PS F measurement sample but with different particles. The R I T C dyed silica particles described in Section 2.3.6 $\left(\varnothing_{\text {fluo }}=77 \mathrm{~nm}(4 \%), \varnothing_{\text {total }}=354 \mathrm{~nm}(2 \%)\right)$ were repeatedly dried on on a cover glass to build a pile more than $100 \mu \mathrm{m}$ high and incorporated into a channel as described above. This was filled with glycerol/water mixture $\left(n_{21}^{D}=1.4506\right)$ and sealed with U V curing glue. Image stacks were captured in the manner described above for the actin filaments but with $534 \mathrm{~nm}$ excitation.

\subsubsection{Microscopy}

Measurements were performed on a Leica TCS SP8 S TED 3X inverted confocal microscope controlled with LASX version 3.5. This was equipped with a Leica HC PL APO $93 \times / 1.30$ glyc motcorr STED white objective with a correction collar and an $85 \mathrm{w} \%$ glycerol/water mixture $\left(n_{D}^{21}=1.4506, V_{D}^{21}=\right.$ 47.1) as immersion. The axial focal shift due to the small RI mismatch of this immersion with the sample medium should be only $\sim 2 \%$ [160], so the stage displacements are quoted as the $z$ heights in this chapter. For excitation, a $543 \mathrm{~nm}$ laser line $(80 \mathrm{MHz}$ pulsed white light laser, NKT Photonics) was used and a $660 \mathrm{~nm}$ continuous wave laser provided the depletion light in STED measurements. Fluorescence emission was detected in the range $553-650 \mathrm{~nm}$ by a hybrid detector. All microscopy was carried out at $23.5^{\circ} \mathrm{C}$. 

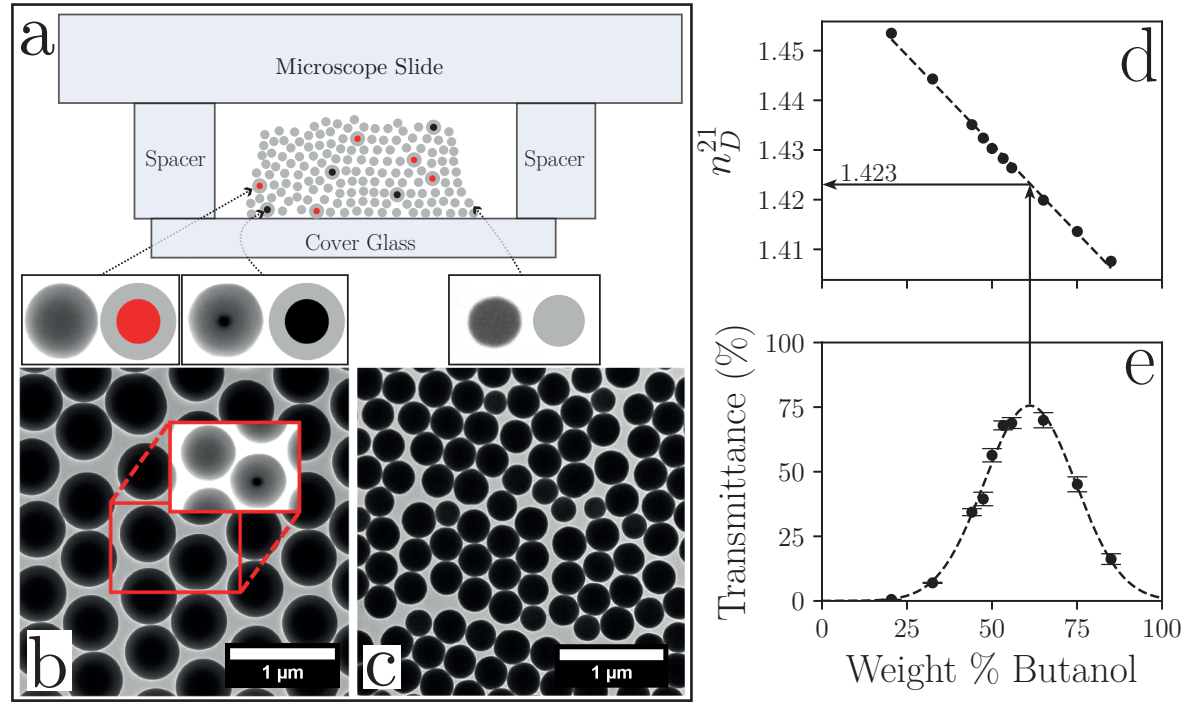

Figure 4.3: $(\mathrm{a}-\mathrm{c})$ Ternary particle system used for PSF measurements. a) Schematic of the cell showing the fluorescent and gold-core particles (red and black centres respectively) suspended in the scaffold of 'undyed' particles (no heterogeneous core). The cell is imaged through the bottom cover glass. b) TEM image of the mixture of fluorescent and gold core particles. The increased contrast in the inset allows the gold core to be seen. Shown above are the particles next to their corresponding schematic form. c) TEM image of the scaffold particles at the same scale and accompanying schematic. (d-e) Determination of the refractive index of the scaffold particles. d) Refractive index of glycerol/butanol mixtures and linear fit with gradient $7 \times 10^{-4}$ and intercept 1.467 . e) Transmittance at $550 \mathrm{~nm}$ of dispersed scaffold particles in glycerol/butanol mixtures and Gaussian fit. The horizontal axis is shared and the arrows indicate index matching (maximum transmittance) at $n_{D}^{21}=1.423$ and $61 \%$ butanol. 
4 Deep STED microscopy: experimental PSFs and deconvolution

\begin{tabular}{|c|c|c|c|l|c|}
\hline & $\begin{array}{c}\text { Sampling } \\
x, y \times z(\mathrm{~nm})\end{array}$ & $\begin{array}{c}\text { Dimensions } \\
x, y \times z(\mu \mathrm{m})\end{array}$ & $\begin{array}{c}\text { PD T } \\
(\mu \mathrm{s})\end{array}$ & \multicolumn{1}{|c|}{ Averaging } & Figures \\
\hline Reflection & $20 \times 80$ & $2.6 \times 4.1$ & 3.8 & SAv $(10)$ & $4.7,4.8,4.13$ \\
\hline PSF & $20 \times 98$ & $10.4 \times 4.1$ & 0.3 & FAc $(4)$, LAv $(2)$ & $4.4,4.8,4.12,4.13$ \\
\hline Bio. Test & $33 \times 80$ & $16.7 \times 5.1$ & 0.3 & LAv $(16)$ & $4.14,4.15,4.16$ \\
\hline Silica Test & $31 \times 80$ & $8.0 \times 5.1$ & 0.6 & FAc $(2)$, LAv $(8)$ & $4.17,4.18$ \\
\hline
\end{tabular}

Table 4.1: Imaging conditions in this chapter. Key: PDT $=$ Pixel Dwell Time, $\mathrm{L} / \mathrm{F} / \mathrm{SAc}=$ Line/Frame/Stack Accumulation, $\mathrm{L} / \mathrm{F} / \mathrm{SAv}=$ Line/Frame/Stack Averaging.

\section{STED alignment and examining the deep depletion spot}

Before imaging the fluorescent particles, the reflection signals (at 543 and $660 \mathrm{~nm}$ ) from the gold core particles were used to find optimal parameters for $3 \mathrm{D}$ STED imaging. First, the depletion beam was adjusted to centre the excitation and depletion reflection patterns (cyan and magenta respectively in Figure 4.8) of a gold-core particle located on the cover glass. This alignment was not adjusted again. Gold core particles were found at $20 \mu \mathrm{m} z$ intervals up to $100 \mu \mathrm{m}$ deep in the sample and stack averaged $3 \mathrm{D}$ images captured of each of them (see Table 4.1). A gold core particle $80 \mu \mathrm{m}$ above the cover glass was repeatedly imaged in the same way with different settings of the correction collar. These data are presented in Figure $4.7 \mathrm{a}$ and b. Based on these results, the following procedure was devised to optimise STED imaging.

A gold particle at each $z$ position was located and a correction collar $(\mathrm{CC})$ position chosen to equalise the $3 \mathrm{D} \mathrm{STED}$ axial lobe intensities as viewed in the $x z$ plane through the centre of the particle. This was achieved by iteratively adjusting the $\mathrm{CC}$ position, re-centring the particle in the field of view by moving the sample stage and checking the intensities of the side lobes with a line profile along $z$ through the $x y$ centre of the particle. With these equalised as much as possible, the reflection images shown in the bottom row of Figure 4.8 were captured, using the parameters in Table 4.1 and CC positions in Figure $4.7 \mathrm{~d}$.

\section{PSF Measurement}

Image volumes of the fluorescent beads were captured from which to extract the PSFs. At each height, test images using low laser power were captured at different $x y$ positions to discern whether or not any fluorescent beads were located in the region of interest, away from the edges. If so, the position was marked and eventually $7-15 x y z$ stacks of dimension $10.4 \times 10.4 \times 4.1 \mu \mathrm{m}$ were captured at the various chosen $x y$ positions in a 'Mark and Find' acquisition. 
The checking was required to avoid wasted image volumes containing no usable particles and it was done in this way to avoid bleaching the fluorescent cores before the main measurement. The low pixel dwell time $(0.3 \mu \mathrm{s})$ used was to ensure that the beads did not bleach significantly during acquisition.

\subsubsection{Analysis}

\section{Reflection Images}

Profiles were extracted for each channel of the image volumes of gold particles by summing the intensities in each slice to give a single value at each $z$ height. Single and double Gaussian functions were fitted to the excitation and depletion profiles respectively. The mean positions and heights of these were used to compare the positions and intensities of the excitation spots and depletion lobes.

\section{PSF Extraction}

The beads in the image volumes at each height were located and summed together to give an averaged image of the particles at that height (Figure 4.4). Only signals well separated from each other and from the image volume boundaries were included. This average was then deconvolved with a sphere of diameter $45 \mathrm{~nm}$ (the known fluorescent core size), yielding the measured PS F [164]. Both of these steps were carried out using Huygens Professional deconvolution software (version 18.10, SVI).

\section{Deconvolution}

All deconvolutions in this chapter were carried out using Huygens with its Classic Maximum Likelihood (CM LE) algorithm. The regularisation parameter ('signal to noise ratio') was set at 20 and 7 for confocal and STED images respectively. This value was not necessarily optimal but was kept constant to allow comparison of restorations using different PSFs. Theoretical PSFs were calculated using the same software with measured solvent refractive indices and the nominal numerical aperture as inputs.

\section{Synthetic Images}

A synthetic image volume was generated starting from a ground truth distribution representing microtubules from Reference 151. This was convolved with a measured 3D STED PSF (see Figure 4.8, $0 \mu \mathrm{m}$ ) and Poisson noise with 


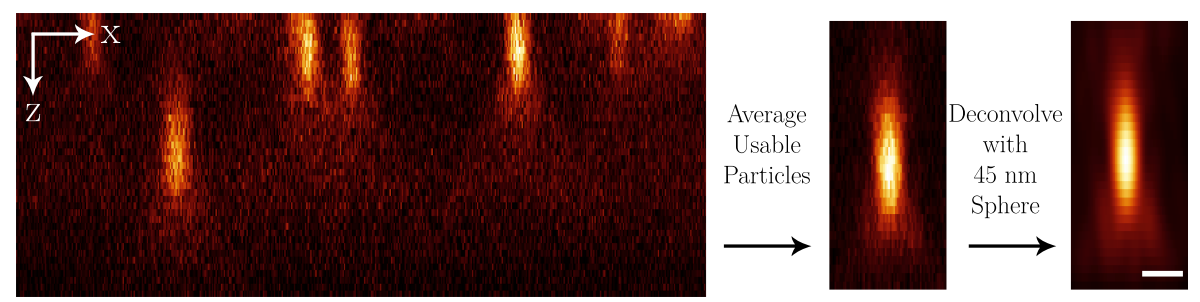

Figure 4.4: Extracting a measured PSF. Particles sufficiently separated from edges and each other are selected from the raw image volume (left) and averaged to improve signal (middle). The PSF is then extracted from this averaged image volume by deconvolution with a sphere the size of the fluorescent core. These images show the sum of pixel values along the y axis, meaning that the lateral separation of particles appears reduced. Nonetheless, the particles 'touching' the top of the image volume were not used. The images have the same scale and the bar has length $500 \mathrm{~nm}$.

signal to noise ratio ( $\mathrm{SNR}$ ) of 7 added, also using Huygens. This procedure is summarised in Figure 4.5.

\subsection{Results and Discussion}

\subsubsection{Behaviour of the depletion beam far from the objective}

Part of the standard operating procedure in STED microscopy is inspection and alignment of the excitation and depletion focal spots, conveniently achieved using the bright reflections of gold nanoparticles on the coverslip [130]. However, for imaging deep in the sample, good alignment at the bottom does not guarantee the same at deeper imaging planes. The sample described in Section 4.3.1 has gold core particles dispersed in $x, y$ and $z$, allowing inspection of the STED pattern at various heights. The changes to the focal spot with increasing imaging depth are examined in this section, along with a method to counteract them.

\section{Axial lobe asymmetry}

The effect of increasing imaging depth is immediately visible in Figure 4.6 b: the axial S TED lobes become increasingly lopsided until the lower one is barely present. This is quantified by the line profiles on the left of Figure $4.6 \mathrm{a}$ and 

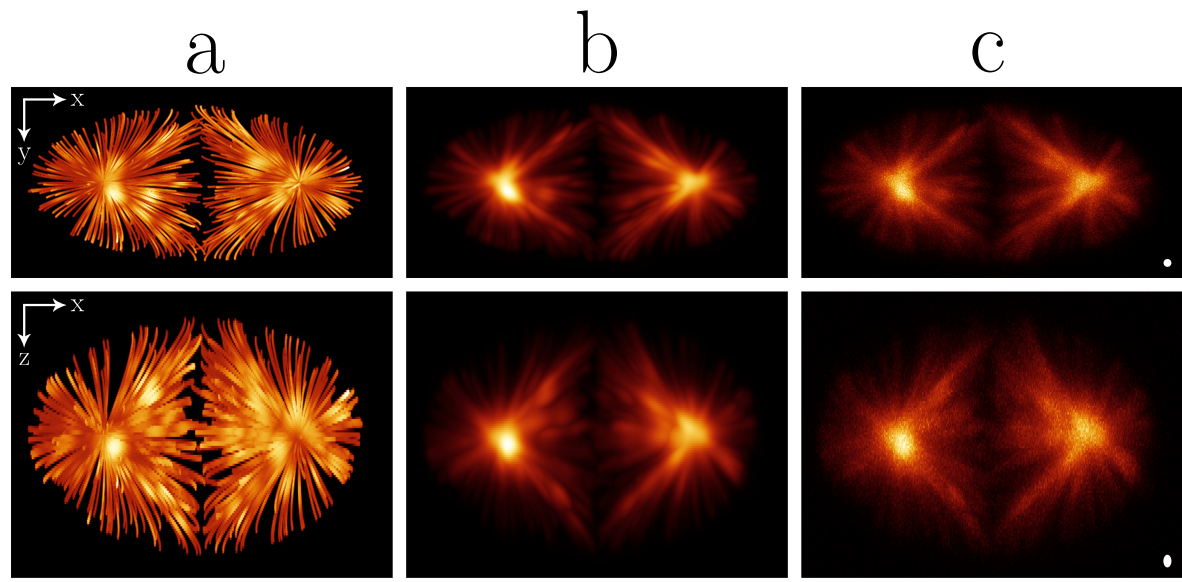

$$
f(x, y, z)
$$

$$
f(x, y, z) * h(x, y, z)
$$

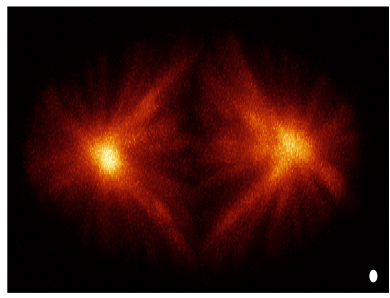

$$
\begin{gathered}
f(x, y, z) * h(x, y, z) \\
+N(x, y, z)
\end{gathered}
$$

Figure 4.5: Simulated image formation. a) Ground truth object from Reference 151. $f(x, y, z)$ is the distribution of fluorophores in $3 \mathrm{D}$ space. b) Convolution of the object with the PSF $(h)$. c) Poisson noise is added to the convolution to simulate photon noise. All images are maximum projections through the volume. The white spots on the right indicate the extent of the PSF at half maximum.

the results of fitting these profiles shown in Figure $4.7 \mathrm{a}$. In the latter, the red crosses show the difference in height of the two components of the normalised double Gaussian function used to fit the STED line profile. The upper lobe becomes increasingly dominant before plateauing high in the sample.

This is a consequence of the refractive index mismatch [136] and may be intuitively understood by considering the way the STED focal spot is formed. The 3D STED beam is shaped using an annular phase plate, which has the effect of subtracting a high N A focal spot from a lower N A focal spot [128, 129]. Both of these spots grow with depth in the sample due to spherical aberration, increasing the axial width of the dark region between the STED lobes. This effect was observed but omitted from Figure 4.7 for clarity.

Depth dependent spherical aberration is more severe with higher N A [135]. The observed pattern may therefore be explained by more rapid asymmetric aberration of the smaller (subtracting) PSF, such that eventually the entire bottom half of the larger spot is negated. 


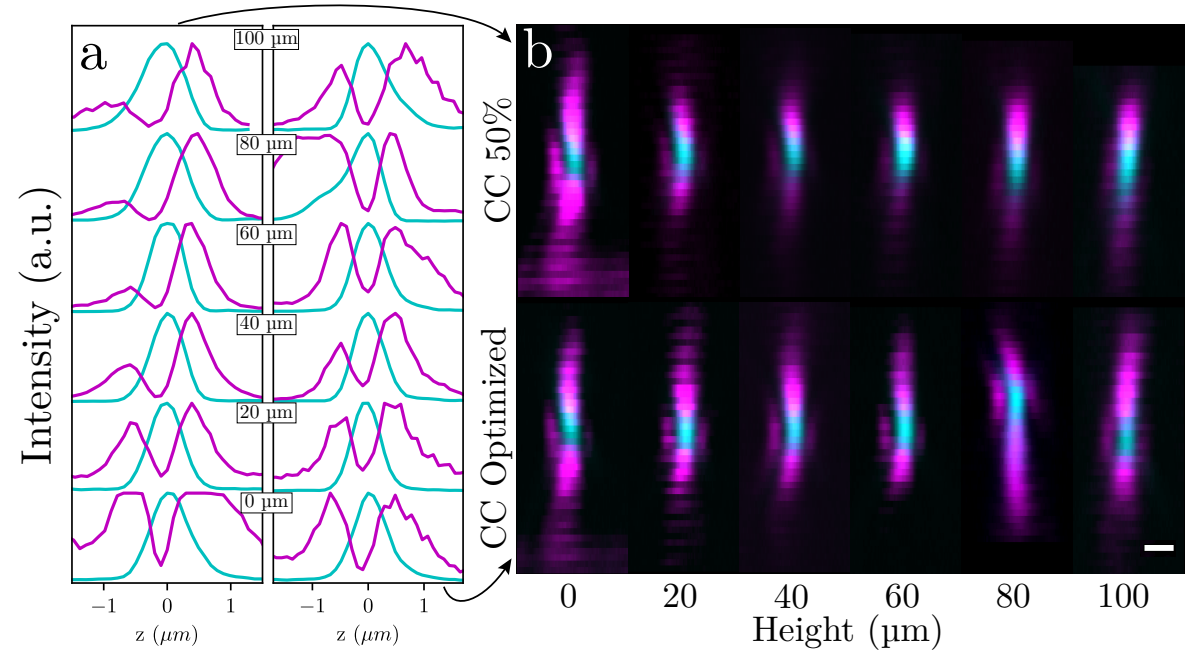

Figure 4.6: The changing STED pattern deep in the sample. a) Line profiles along $z$ for the excitation (cyan) and depletion (magenta) focal spots. The left column corresponds to the top row in (b), in which the correction collar was at $50 \%$ throughout. In the right column (bottom row in (b)), the correction collar was adjusted to equalise the axial STED lobes. (b) $x z$ slices of the excitation and depletion spots with the same colouring as in (a). In the top row the correction collar was fixed while in the bottom, it was adjusted at each height. The scale bar has length $500 \mathrm{~nm}$.

\section{Depletion misalignment}

Also visible in Figure $4.7 \mathrm{a}$ is a related effect: the increasing axial misalignment of the excitation and depletion spots. The centre of the STED pattern became linearly higher with respect to the excitation spot for deeper imaging. Axial shifts of similar magnitude have previously been observed, although not explained, at lower depth in a system of greater R I mismatch [138]. An explanation may be found using geometric optics of two rays of different wavelength passing through the dispersive immersion media, which predicts a misalignment, $\Delta F^{\prime}$, as a function of the axial stage displacement, $\Delta z$ :

$$
\Delta F^{\prime} \approx \Delta z\left(\frac{n_{3}\left(\lambda_{s}\right)}{n_{1}\left(\lambda_{s}\right)}-\frac{n_{3}\left(\lambda_{e}\right)}{n_{1}\left(\lambda_{e}\right)}\right)
$$


$n_{1}$ and $n_{3}$ denote the refractive indices in the lens and sample immersion media at the depletion $(s)$ and excitation $(e)$ wavelengths. Equation 4.7 and the measured dispersions (Table 4.2) were used to plot the theoretical line in Figure 4.7 a. The details of this simplified model as well as the approximations leading to Equation 4.7 may be found in Appendix 4.A.

\section{Correction collar effects}

Both the dimming of the lower lobe and the increasing misalignment are undesired for STED microscopy; the former will pass signal which is unwanted and the latter will reject that which is useful. Figure $4.7 \mathrm{~b}$ shows an attempt to counteract these effects by adjusting the correction collar of the objective lens. Imaging at $80 \mu \mathrm{m}$, the correction affected the same properties as changing the imaging depth, namely the balance of the STED lobes and the axial misalignment of the two focal spots.

The axial misalignment was fitted with two straight lines, one rising and one falling. The linear relationship is not possible to explain without knowledge of the internal lens design so it was concluded that the correction element was designed to effect a linear shift in focal position which was made unequal by the same dispersive media discussed above. Similarly, it is assumed that the correction element is designed to shift the point at which the lens is fully achromatic, allowing adjustment of the STED lobe balance. Limited understanding of the action of the correction element does not hinder its utility in counteracting the problems introduced by deep imaging.

Ideally, the $\mathrm{CC}$ would be able to reduce both the STED lobe disparity and axial misalignment to 0 . A minimum in axial lobe disparity was found with the $\mathrm{CC}$ at around $20 \%$. At the same setting, a turning point in the axial misalignment was observed, although not a minimum in magnitude. This agreement in stationary point location is promising in that one $\mathrm{CC}$ setting should be able to reverse both of the problems introduced by the deep imaging plane. Neither the lobe disparity nor the axial misalignment reached zero at this setting, suggesting a systematic misalignment which should have been corrected for by adjustment of the depletion beam path behind the objective. This last conclusion is speculative based on this plot, but is supported by the discussion of Figure $4.7 \mathrm{c}$ below.

\section{Using the correction collar for deep STED imaging}

The $\mathrm{CC}$ was adjusted at each height to equalise the STED lobes as much as possible (Figure $4.7 \mathrm{c}$ ). With the STED alignment properly adjusted behind the objective, it was possible to keep both the misalignment and lobe disparity close 

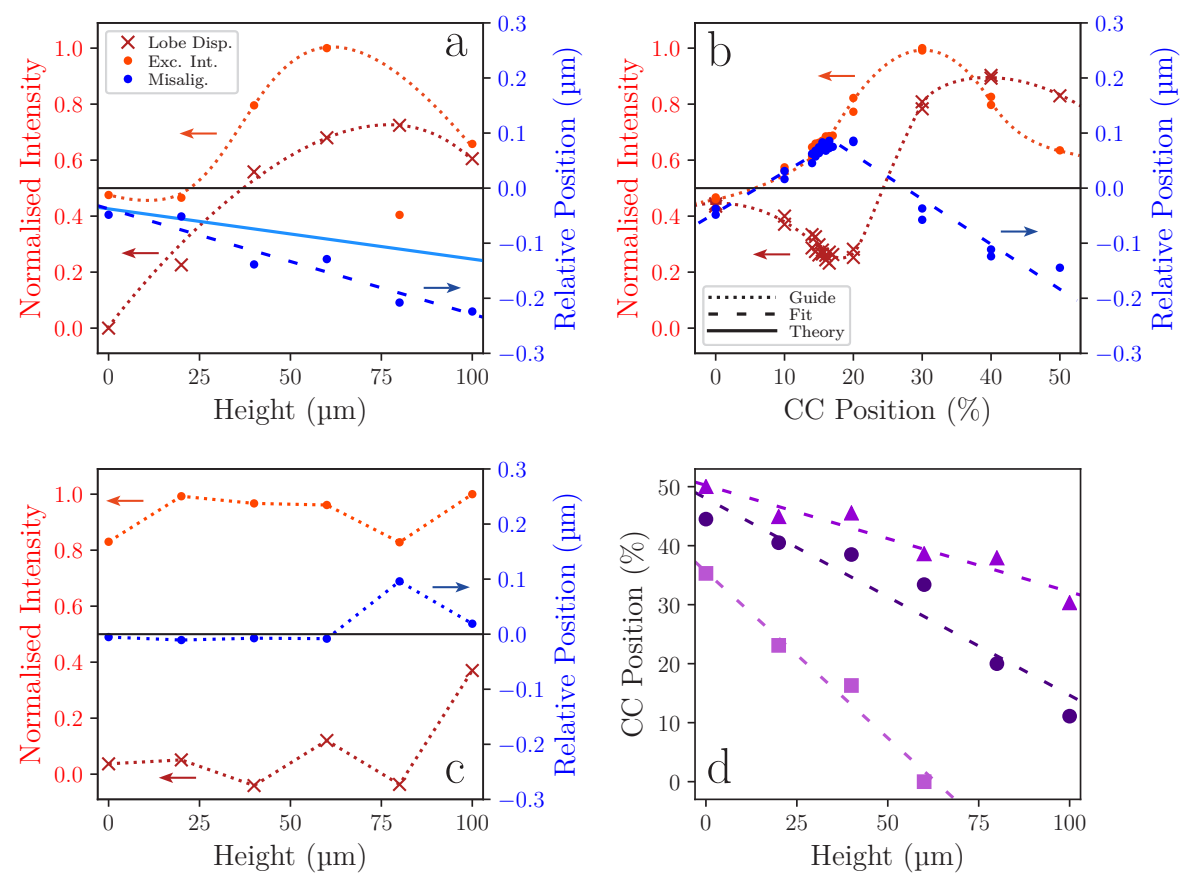

Figure 4.7: Effects of depth and the correction collar, as observed in reflection. In $\mathrm{a}-\mathrm{c}$, the left axis shows the relative maximal intensity (Gaussian fit height) of the excitation spot as well as the intensity difference between normalised STED maxima (between Gaussian fit heights). On the right is the height of the excitation spot measured relative to the centre of the axial depletion lobes. The long dashes indicate fits and the short dashed lines are guides for the eye. For 'ideal' conditions, both the axial misalignment and the STED lobe disparity would be 0 , and the excitation intensity maximal. The legend in (a) applies to panels $\mathrm{a}-\mathrm{c}$ and that in (b) applies to all panels. a) CC fixed at 50\%. The theoretical line is based on the relative dispersion of the immersion media, calculation detailed in Appendix 4.A. b) Height fixed at $80 \mu \mathrm{m}$. The axial misalignment was fitted with two straight lines, sharing the points at $20 \%$. c) $\mathrm{CC}$ adjusted at each height to equalise the STED lobe intensities. d) The correction collar was adjusted to equalise the axial lobes in three runs on different days in the same test sample. Here, the colours and symbols serve only to distinguish the three runs. 
to 0 . Examples of the observed depletion patterns with the $\mathrm{CC}$ progressively optimised are shown in the bottom rows in Figures $4.6 \mathrm{~b}$ and 4.8 . The $\mathrm{CC}$ values which were required in three different experiment runs are shown in Figure $4.7 \mathrm{~d}$. The gradients of the linear fits have an order of magnitude consistent with that predicted by combining the counteracting axial shifts in Figures $4.7 \mathrm{a}$ and b but are imprecise. This despite all three runs being done with the same test sample on the same microscope. More disconcerting still is the run in which the $\mathrm{CC}$ was not able to correct for heights beyond $60 \mu \mathrm{m}$. The changes in gradient in this plot are not due to changes in the measurement temperature as this was constant at $23.5^{\circ} \mathrm{C}$, leaving the reasons for these changes unknown. It is possible that temperature and usage cycles in the weeks between these measurements had residual effects on the correction element or other parts of the optical train. Just as the required $\mathrm{CC}$ position is rarely known in advance in conventional imaging, the line of $\mathrm{CC}$ values for deep STED must also be determined immediately prior to measurement. That it is a line though, should allow the practising microscopist to simply check the optimal $\mathrm{CC}$ positions at the minimal and maximal required image heights and interpolate between them.

In an attempt to find a more convenient method to optimise the $\mathrm{CC}$ position at each height, the intensity of the excitation spot was examined, and its value plotted in Figures $4.7 \mathrm{a}-\mathrm{c}$. The manufacturer-recommended procedure for choosing a $\mathrm{CC}$ position in conventional microscopy is to maximise the reflection of the excitation beam in some object, often the cover glass interface [165]. However, the excitation maximum occurred at a different $\mathrm{CC}$ position than the optimal STED pattern. This may also be attributed to the dispersion properties of the immersion media discussed in Appendix 4.A. There it is shown that the STED beam experiences a slightly larger R I mismatch, meaning that its position of minimal spherical aberration was different to that of the excitation beam. It was decided to use the optimal depletion condition in the following measurements based on the idea that a brighter excitation could only counteract the loss of signal due to axial misalignment, not recover the loss of resolution due to one-sided depletion. Verification of this reasoning remains for future work.

Thus was the procedure for the following measurements determined: first optimise the STED pattern in reflection and then use the chosen CC positions for imaging at different depths.

\subsubsection{The measured PSF}

The experimentally measured PSFs for confocal and 100\% 3D STED imaging are shown in Figure 4.8, along with reflections of the corresponding focal spots. 


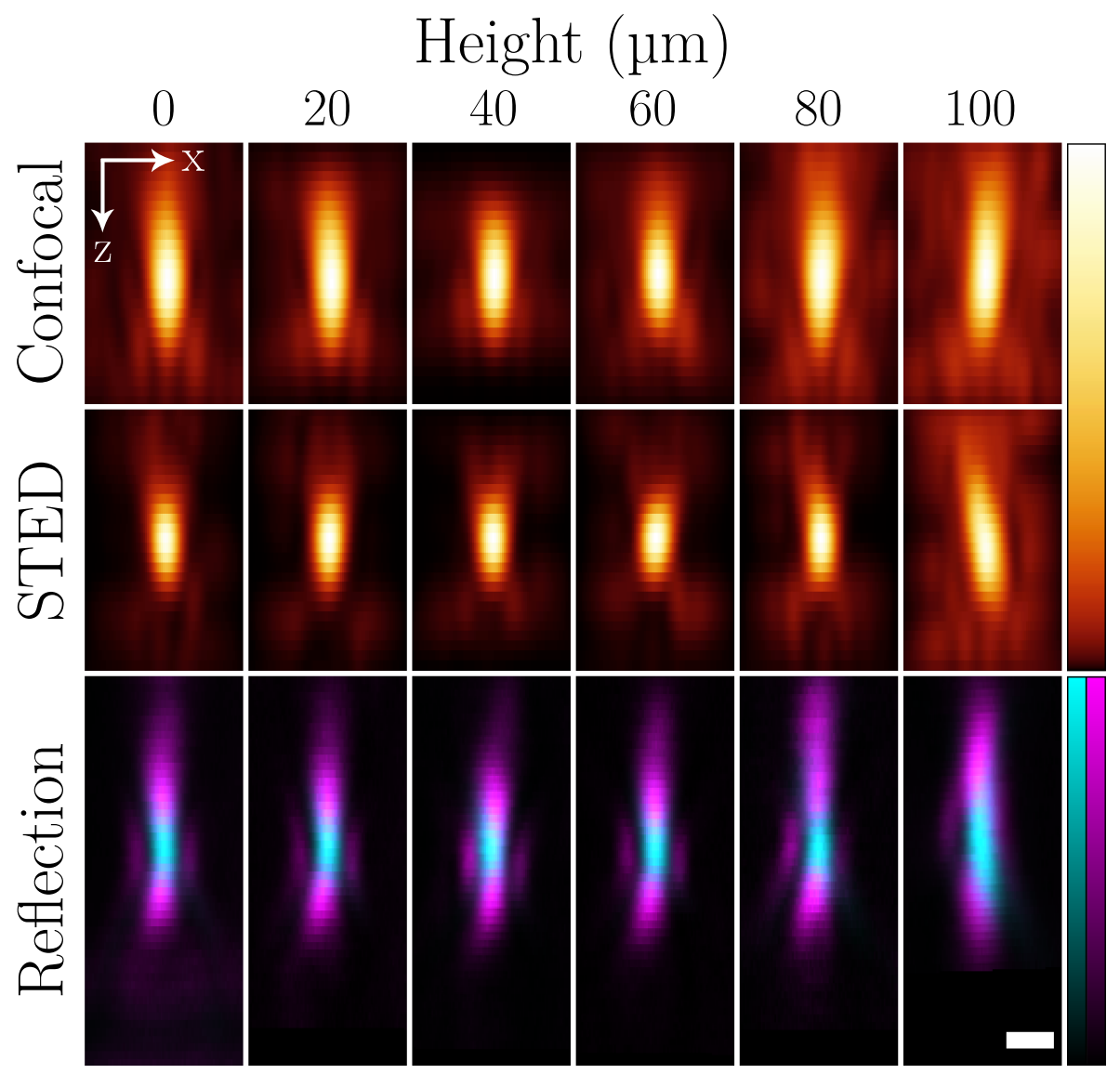

Figure 4.8: Measured PSFs in Confocal (top) and 3D STED (middle) imaging modes at the 6 indicated heights above the cover glass. The first column is comparable to the theoretical PSFs in Figure 4.1. The colourbar on the right shows linearly increasing grey values displayed with the same lookup table $(\gamma=0.5)$ as the PSFs. The bottom row shows the reflection of the excitation (cyan) and depletion (magenta) beams at the same heights $(\gamma=1$, see Figure $4.13 \mathrm{~d}$ for a mixing colourbar). All images have the same scale and the bar has length $500 \mathrm{~nm}$. The relative positions of the separate images is not physical and was chosen to align the signal centres as much as possible without cropping any significant intensity. 


\section{On the measurement itself}

The PSFs were measured with details besides the on axis maxima visible in confocal and STED up to at least $60 \mu \mathrm{m}$. The secondary lobes are present below the centre in agreement with the theory in Figure 4.1. Higher order maxima were lost below the sensitivity of the measurement (the images shown were cropped from a larger imaged area due to the lack of signal beyond the displayed extent). Lobes above the centre were also not present, a known effect of immersion-medium R I mismatch [135]. Higher in the sample, the central maximum is clearly visible but the outer lobes are lost in the high background. While sufficient for microscope quality control, the lack of detail in the higher PSFS will negatively affect deconvolution. The deterioration is attributed to the decrease in signal high in the sample due to the R I mismatch $(\Delta \mathrm{n}=0.027)$. This does not imply that imaging is not possible at these heights, merely that obtaining an accurate $\mathrm{PSF}$ in $3 \mathrm{D}$ is challenging. The latter requires much more signal from much dimmer objects than the former.

Thus it is concluded that the measurement broadly reproduces the PSF shape predicted by theory (Figure 4.1) but to check the precision, separate measurement runs were compared. The results are presented in Figure 4.10. It can be seen that the STED results are fairly consistent for the two runs with the axial PSF width generally maintained at around $550 \mathrm{~nm}$ throughout the measured depth. The confocal PSF widths are less consistent, with several exhibiting unacceptable widths of greater than $1 \mu \mathrm{m}$. This variation could be due to the setting of the correction collar based on the depletion focal spot. It was found in the previous section that this does not optimise the excitation and it is feasible based on Figure $4.7 \mathrm{~d}$ that the relative effect of the $\mathrm{CC}$ on the two lasers could be different on different days, causing these large fluctuations. A fairer comparison would perhaps be with the CC optimised for the excitation spot for confocal PSF measurements, but this was beyond the scope of this STED-focused work.

\section{On the results}

Considering the measured PSF as a quality control measure of the microscope, the shape and size of the PSF are what matter. The PSFs measured show good axial symmetry, indicating a well aligned microscope, with only a slight tilting visible at $100 \mu \mathrm{m}$. The STED beam is performing its duty in compressing the PSF in the expected manner: the $3 \mathrm{D}$ STED PSF is smaller both axially and laterally than the confocal one (Figures 4.8,4.10,4.11), the $2 \mathrm{D}$ STED one is smallest laterally (Figures 4.9,4.13), and the $60 \% 3 \mathrm{D}$ one is between these two. 


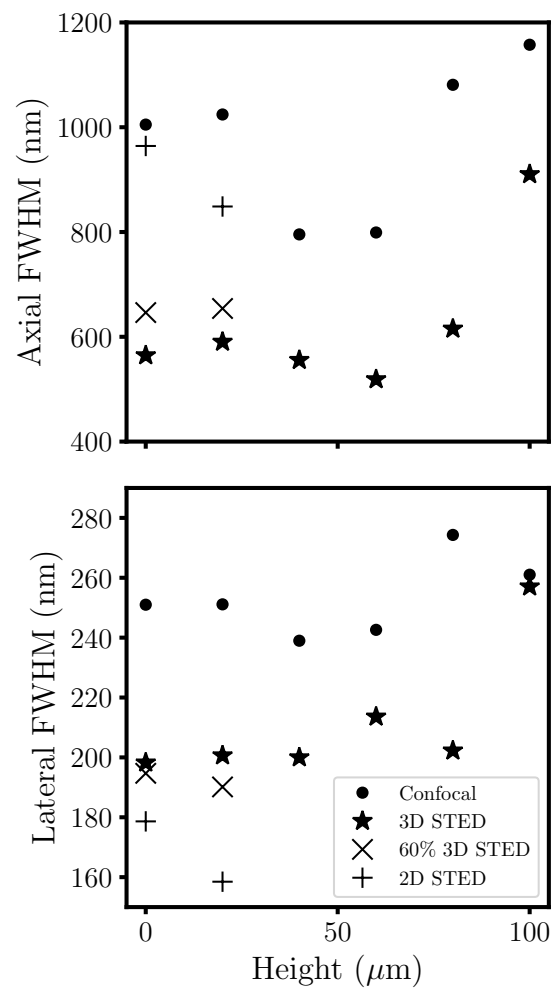

Figure 4.9: Axial (top) and lateral (bottom) full width at half maximum (FWHM) of the measured PSFS on a single day. The horizontal axis is shared and the vertical axes have different scaling.

The expected trend of deteriorating STED PSF with deeper imaging into the mismatched medium is only weakly visible, if at all. As the mismatch is small, this should be a weak effect, and it seems that it is weaker than the fluctuations induced by imprecise CC optimisation, at least lower in the sample.

The PSFs calculated for the same conditions (Figure 4.12) are consistently smaller than the measured ones. This disparity likely results from the PSF calculation assuming an optically homogeneous sample medium, which the measurement sample is not. While the scaffold particles were index matched with the solvent $\left(n_{D}^{21}=1.4230\right)$, the probe particles were not $\left(n_{550}^{21}=1.4303\right)$. The presence of these mismatched particles, albeit at low concentration, must 


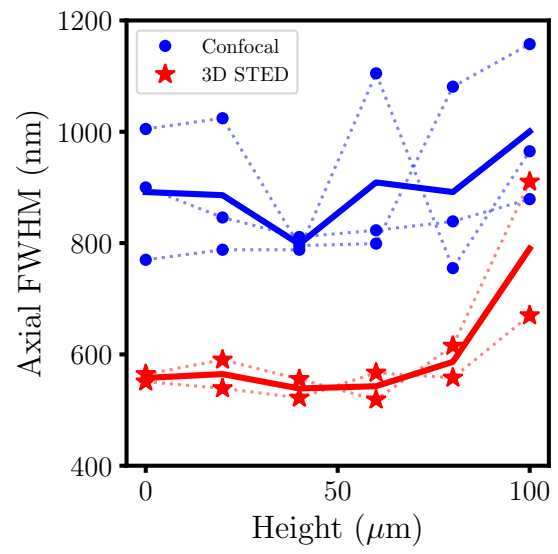

Figure 4.10: Axial FWHM of PSFs from different measurement runs (different days). The dotted lines group the measurements from one run and the solid lines are average values.

have deteriorated the PSF as compared to a completely matched medium.

Distinct from the optical effects on the PSF are possible sources of measurement error which could also have increased the spread of the PSF. These include the vibrations in the microscope and fitting errors when averaging the bead images together.

\subsubsection{Depth Variant Deconvolution}

Two test samples, one biological and one representative of colloidal model systems, were used to test the utility of the measured PSFs for deconvolution.

\section{Biological Test Sample: Caco-2 Cells}

Actin filaments inside the microvilli of $\mathrm{Caco}^{-2}$ cells were imaged under the same microscope conditions and at the same heights as the PSFs were recorded. These were then deconvolved with both a calculated PSF and the experimentally measured one and the results are shown in Figures 4.14-4.16. Representative images of these two PSF types are shown in the top and bottom rows in Figure 4.12. It may be seen that in most cases the results of the two deconvolutions were similar, with some notable exceptions discussed later in this section. 

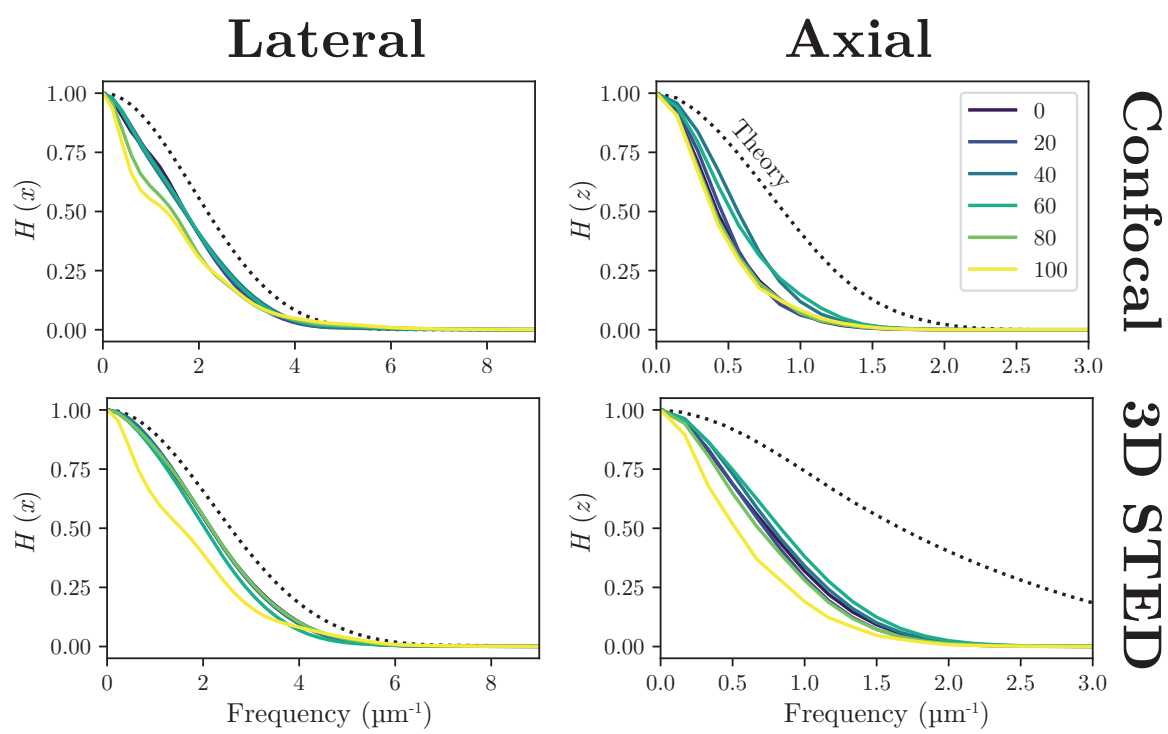

Figure 4.11: Optical transfer functions corresponding to the measured PSFs. The line colours correspond to the depths in the sample according to the top legend. Black lines show the theoretical OTFs. 

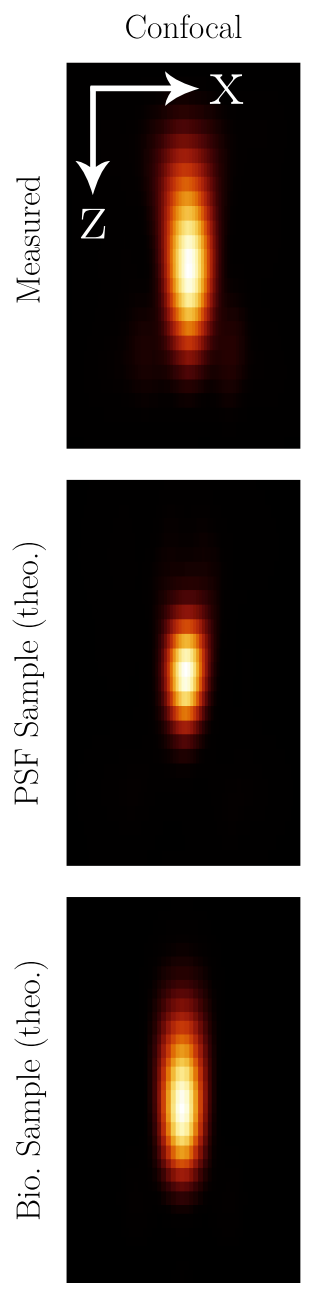
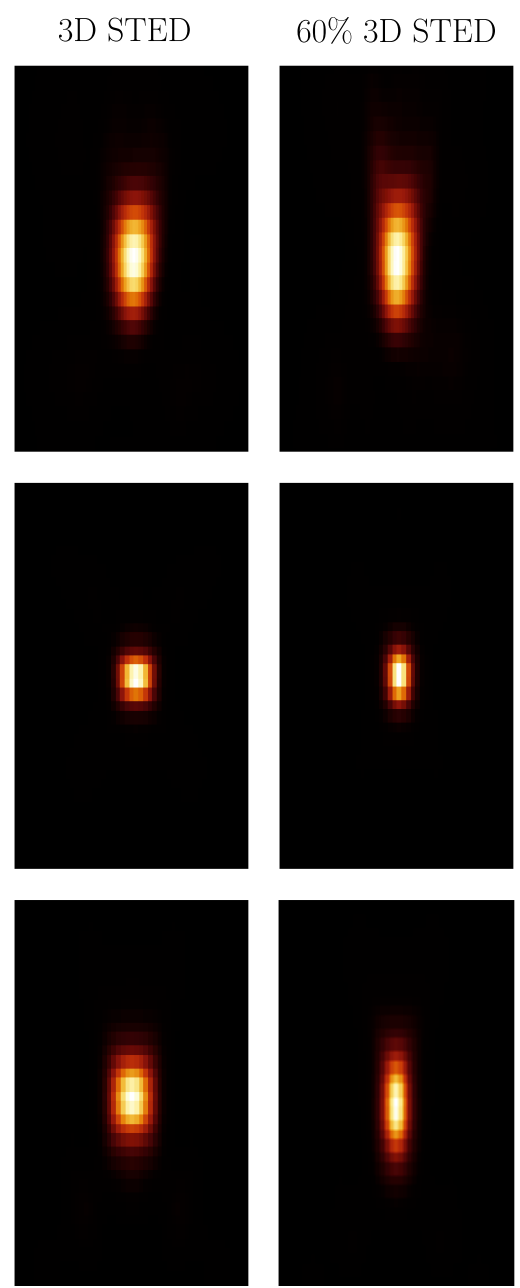

2D STED
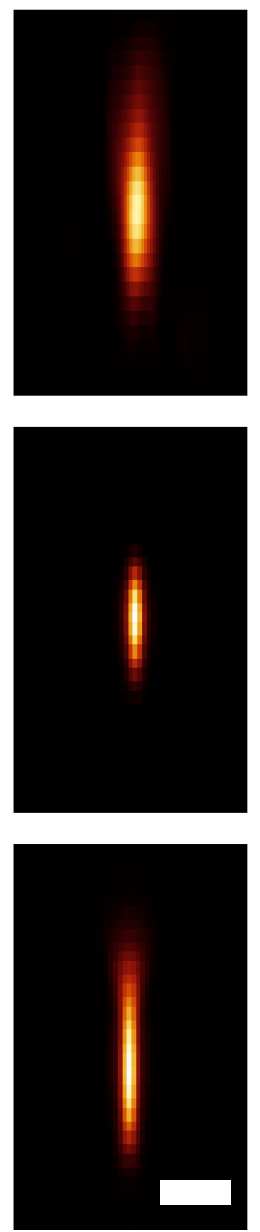

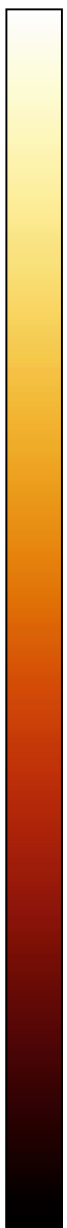

Figure 4.12: Measured and theoretical PSFs at a depth of $20 \mu \mathrm{m}$. The middle and bottom rows show PSFs calculated for the conditions in the PSF measurement $(\Delta \mathrm{n}=0.027)$ and biological $(\Delta \mathrm{n}=0.055)$ test samples respectively. 


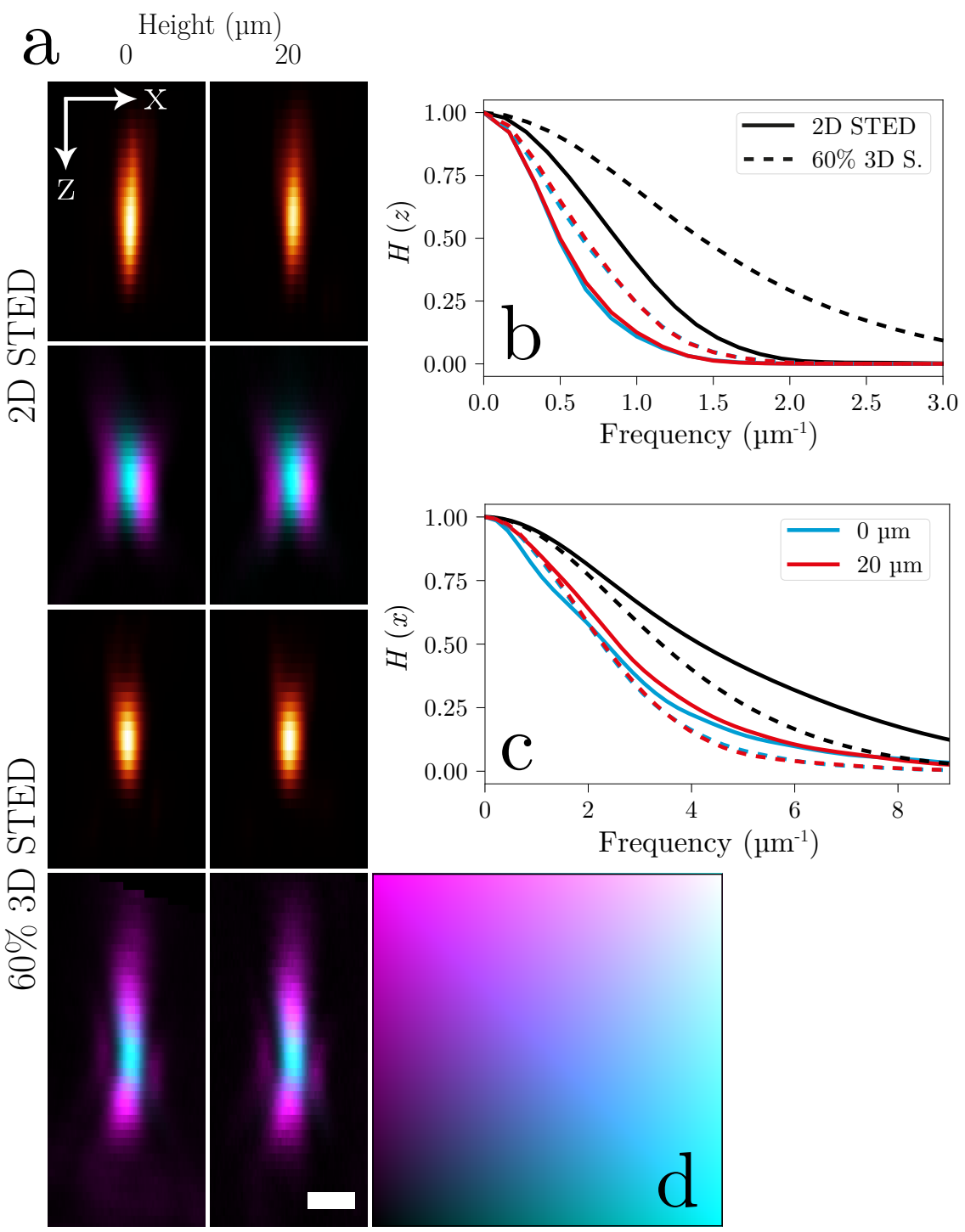

Figure 4.13: Other STED imaging modes. a) PSFs measured in 2D STED (top) and $60 \% 3 \mathrm{D}$ STED (bottom) modes. Shown below each is the reflection signal of the excitation (cyan) and STED (magenta) lasers. (b) and (c) show axial o TFs computed from the measured PSFS in (a). Both legends apply to both plots and the black lines show theoretical predictions. d) 2D colourbar for all mixed reflection signals shown in this chapter. 
Examination of Figure 4.12 (top and bottom rows) reveals that the PSFs used for the two deconvolutions were similar in size and form, although the theoretical ones are slightly smaller. It is unsurprising then that they yielded similar restoration results. The differences between the two theoretical predictions (bottom rows in Figure 4.12) indicate that the two PSFs used for deconvolution may have arrived at this similar form for different reasons.

In Section 4.4.2, it was concluded that the spreading of the PSF was due to the optical inhomogeneity introduced by the probe particles. Here, the large PSFs are predicted by the calculation due to the larger refractive index mismatch between the immersion and sample media. It therefore appears that the two PSFs, and hence the deconvolution results, are so similar due to the coincidence that the different factors disturbing them resulted in spreading of a similar magnitude.

Determination of which restoration is closer to the truth is complicated by the chaotic nature of this biological specimen, for which the ground truth is unknown. A case where the true object form is known (simulated imaging) is discussed in Section 4.4.4. Nonetheless, it is possible to gain some insight by comparison of the restoration results and the knowledge that the microvilli are fibrous with cores separated by about $100 \mathrm{~nm}$ [166]. For example in Figure 4.15 at $20 \mu \mathrm{m}$, the use of the measured P S F resulted in a loss of detail which is clearly visible in the raw image. The theoretical PSF also seems to recover additional fine detail as compared to the measured one, most visible in Figure 4.16. In fact, most of these details are likely spurious ones resulting from noise misidentified as features during deconvolution. This can result from overestimation of the microscope bandwidth during restoration (using a PSF which is too small), a problem discussed further in Section 4.4.4.

It is worth discussing the relative merits of the experimental and theoretical PSFs in relation to this test sample. The theoretical one has the obvious advantage of requiring much less effort to implement, but beyond that it also has the advantage of being able to account for different refractive indices in the optical path. In this case, the index of the mounting medium was 1.395 , rather lower than that of the immersion liquid (1.45). The depth dependence of the PSF shape depends on this difference and so was taken into account during calculation of the PSF. On the other hand, the experimentally measured PSF was restricted to the refractive index of the PSF measurement sample (1.423). It might be possible to scale the measured PSF, but this was not attempted here. Where this scaling approach could be more beneficial is in the case of a distorted PSF due to misalignment in the microscope, where knowledge of the manner of distortion would likely benefit deconvolution, even for very different immersion media.

In fact, when the PSF is distorted is when the measured PSF is likely to 
be most beneficial, allowing the quirks of the microscope to be corrected for. In these measurements, it was found that the PSF was highly symmetric and therefore similar enough in form to the theoretically predicted one to yield similar restorations. Therefore the benefit of measuring the PSF here may be found in: 1) knowing that the microscope is performing in such a way that theoretical deconvolution is reasonable, i.e. quality control and 2) knowing the limit of the microscope to avoid the appearance of spurious features in the final image. This rather than reaching the ambitious goal of pushing the achievable resolution limit downwards. With improved PSF measurements (e.g. with higher signal probes), it may be possible to measure tertiary features of the PSF and hence improve upon the restoration results presented here.

\section{Colloidal test sample: silica spheres}

Figure 4.17 shows images of a partially ordered deposit of fluorescent silica particles alongside attempts at restoration. When touching, these particles have fluorescent cores separated by $290 \mathrm{~nm}$, a distance between the lateral and axial resolution limits of conventional confocal microscopy. This is a better defined test sample than the biological one described above, and also has a refractive index closer to the immersion than either the biological or PSF measurement samples.

The restoration results in Figure 4.17 indicate that the theoretical PSF yielded superior results, with the experimental one more often losing even detail that was visible in the raw images. This is particularly clear in the confocal images at $0 \mu \mathrm{m}$ (top row). This is a trend that continues throughout the image series, although in the noisier STED images, more artefacts are visible in the theoretical restorations. For example: in the $x z$ view of the 3 D STED images at $0 \mu \mathrm{m}$, the theoretical deconvolution resulted in features smaller than the particles in the upper region, presumably formed by noise amplification.

As in the biological case, these results may be explained by considering the PSFS used for the deconvolutions and their relation to the actual system which was imaged. Here, the refractive index match of the test sample was better than that of the PSF measurement sample, meaning that the measured PSF was likely too large: underestimating the resolving power of the microscope in the test sample. This is borne out by the deconvolutions which are overly blurry, because actual detail was assumed to be outside the bandwidth of the instrument. In the theoretical case, this better matching was accounted for, predicting a smaller PSF and being able to restore some real detail. Again it probably overestimated the resolving power by ignoring the inhomogeneity introduced by the particles, leading to some amplification of noise. 


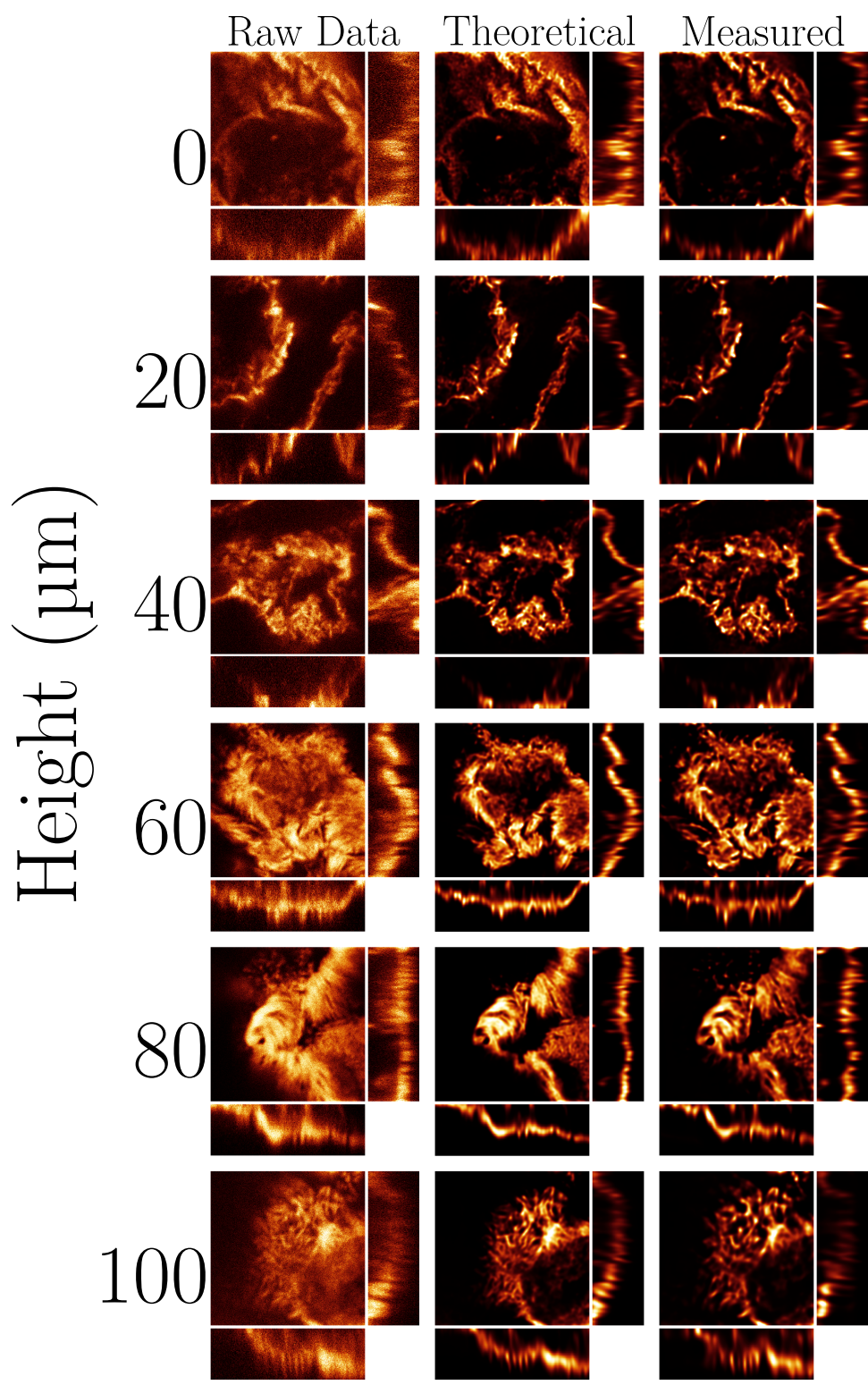

Figure 4.14: Deconvolution of confocal image volumes of Caco-2 cells with experimental and measured PSFs at different depths. These confocal images were recorded after the 3 D-S TE D ones in Figure 4.15, meaning that the SNR may be decreased due to bleaching during the previous imaging. 


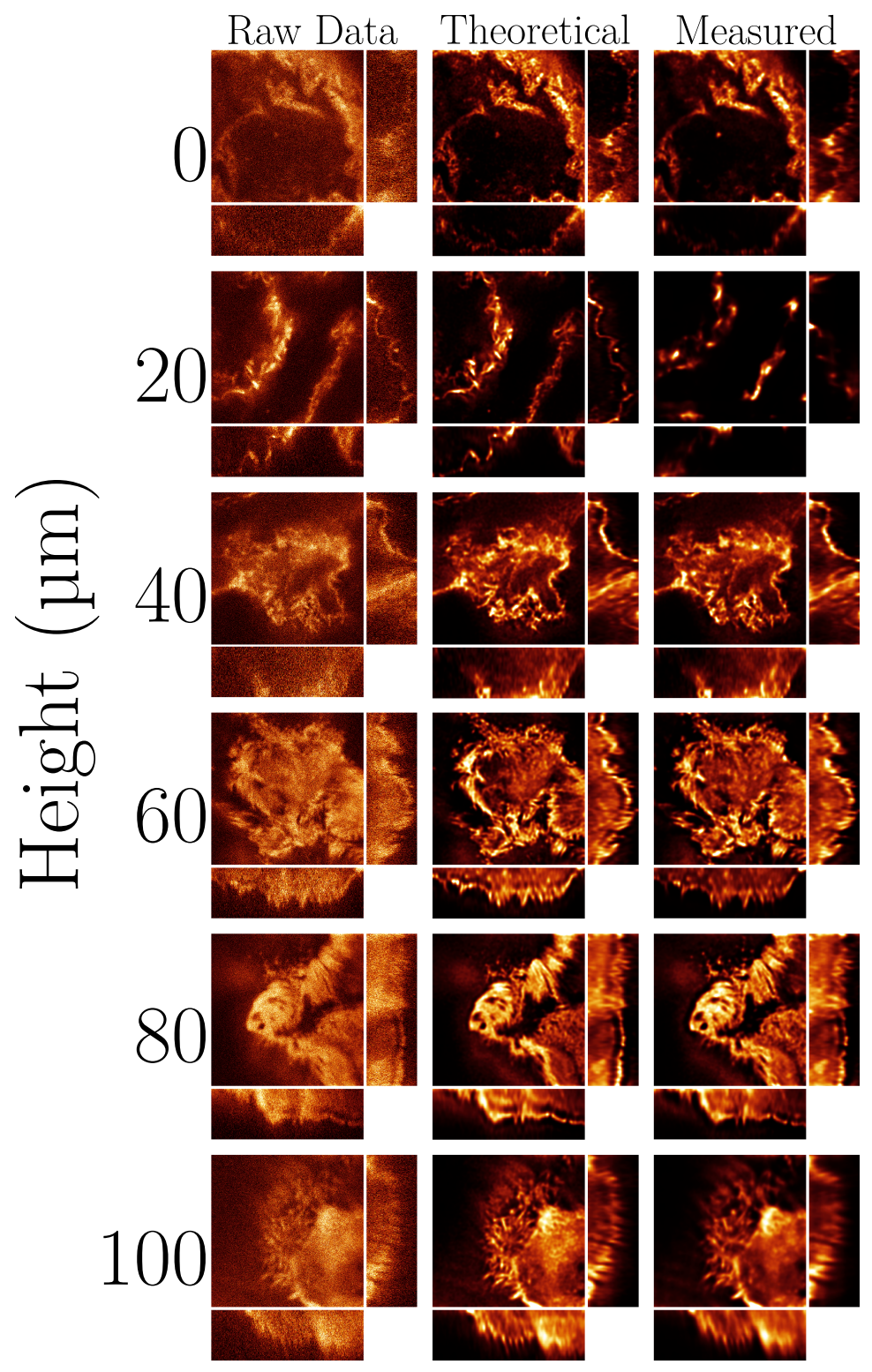

Figure 4.15: Deconvolution of 3D-STED data with the different PSFs. The imaged volumes are the same as those shown in Figure 4.14, allowing direct comparison. 


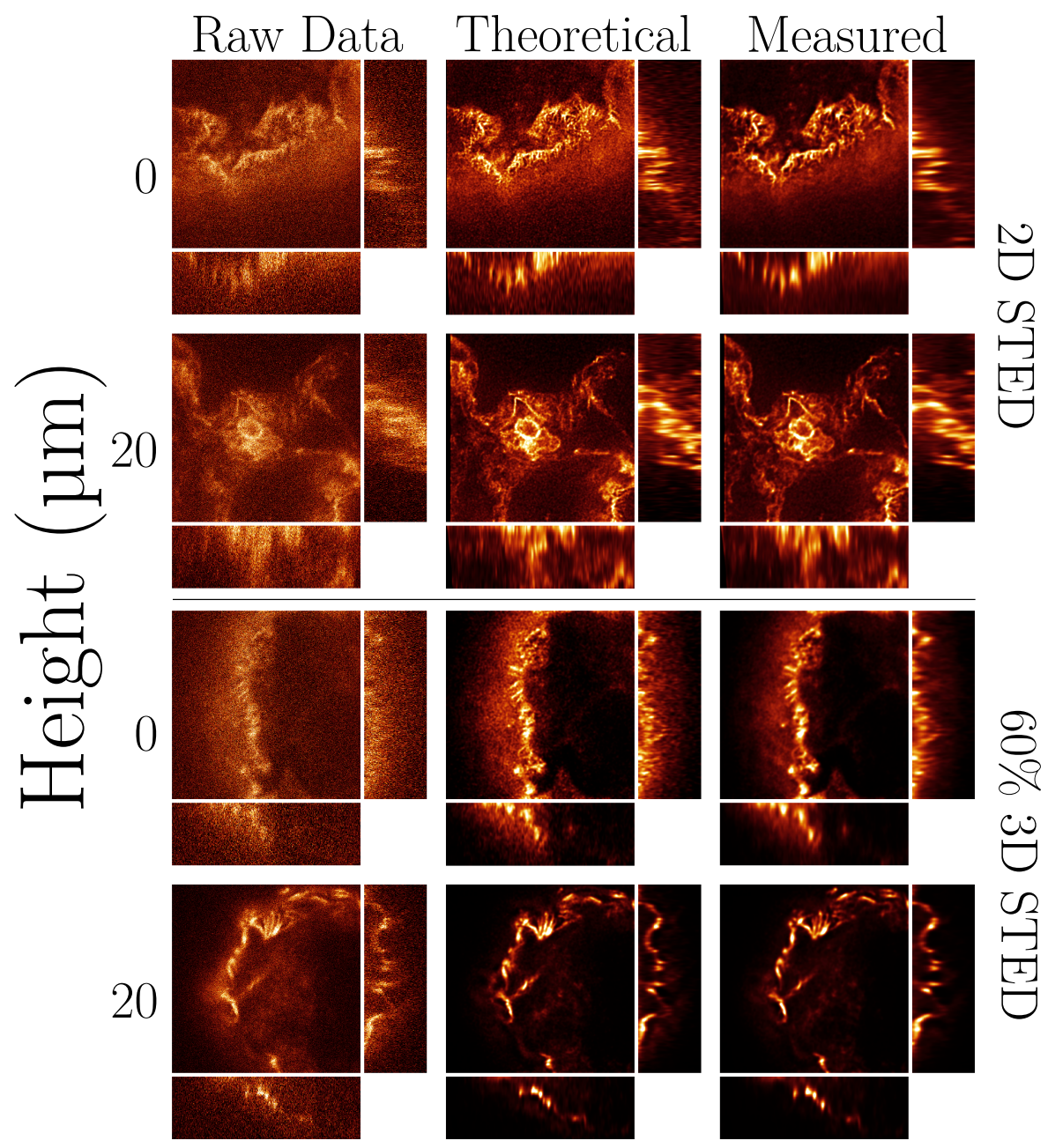

Figure 4.16: Deconvolution of data with different PSFs and with 'less 3D' STED. These data were obtained in unique regions, allowing only indirect comparison of the fibre appearance in each image. 


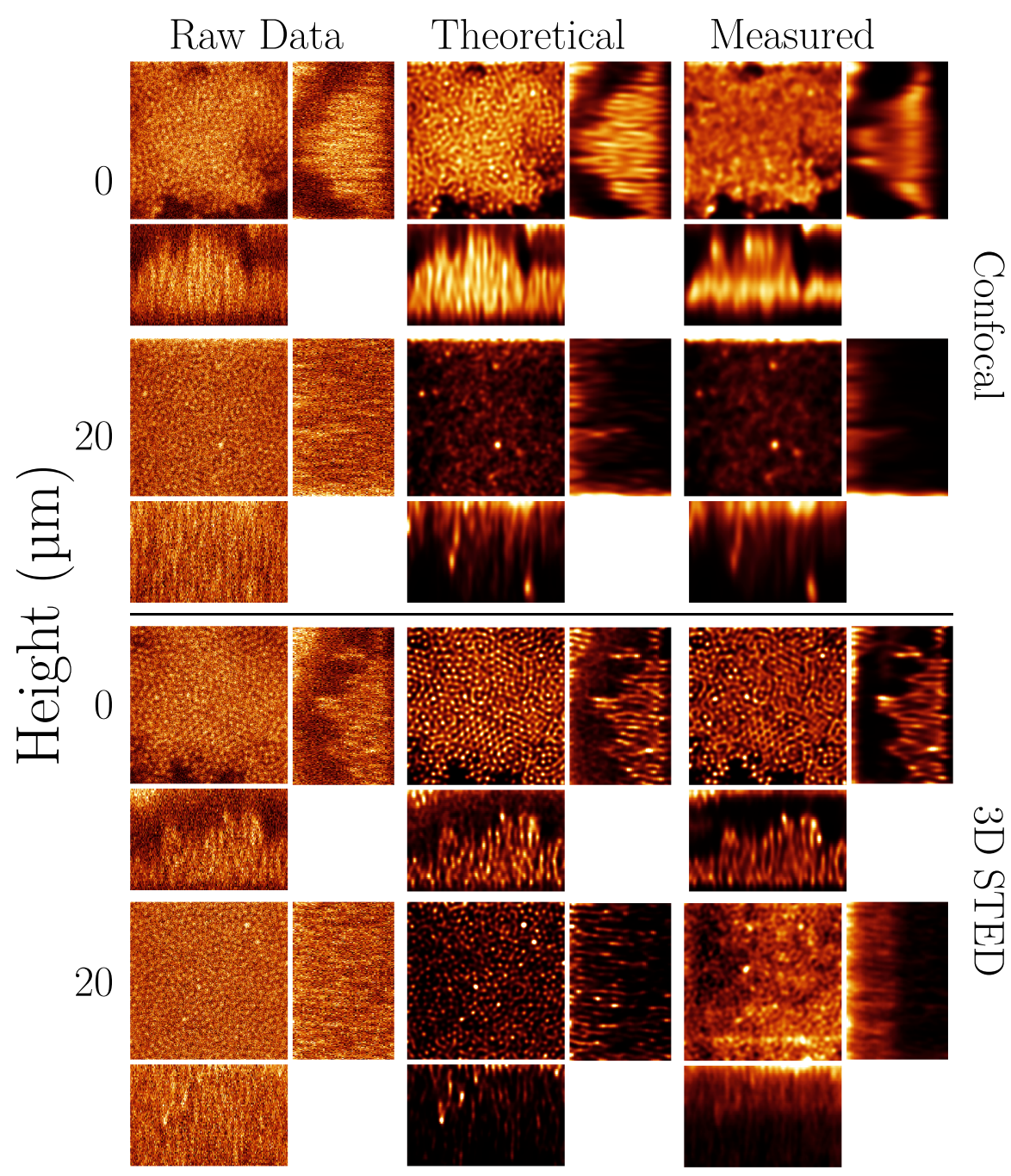

Figure 4.17: Comparison of deconvolution results using colloidal silica particles as a test sample. The confocal (top) and 3D STED (bottom) images were obtained in different regions, allowing only indirect comparison. 


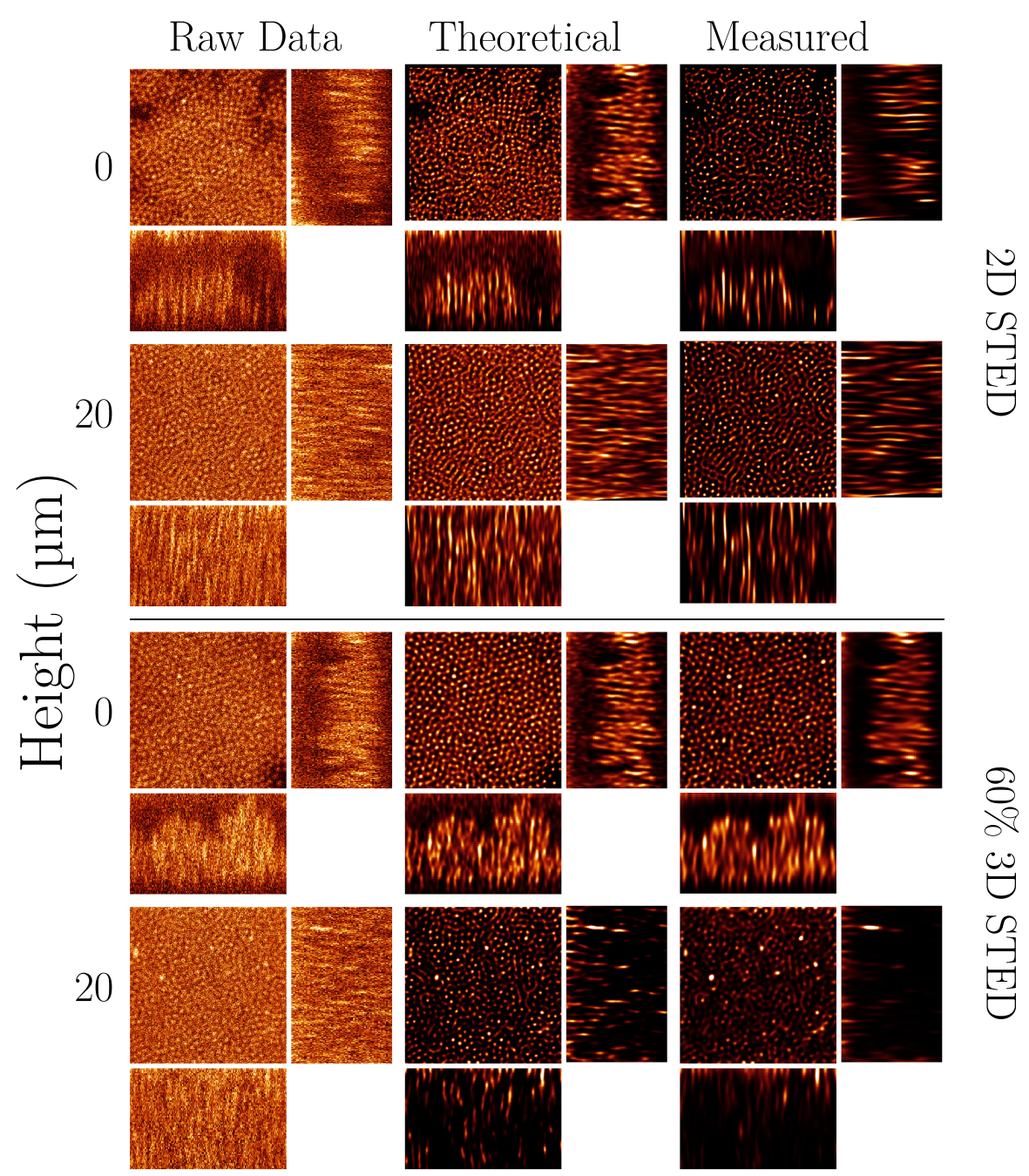

Figure 4.18: Alternative imaging modes of the same sample as in Figure 4.17: $2 \mathrm{D}$ STED (top) and $60 \% 3 \mathrm{D}$ STED (bottom). 


\subsubsection{Deconvolution of synthetic images}

Figure 4.19 shows two deconvolutions of the synthetic microtubule image shown in Figure 4.5. Here a measured PSF was used to simulate the imaging and the same one used for the experimental deconvolution, i.e. the experimental PSF deconvolution used the 'perfect' Ps F. Comparison of restorations of simulated images is much easier because the ground truth image is known.

Examination of the two restored images shows similar differences to those that were observed in the real image deconvolutions above. Again, the theoretical PSF case has added detail such as fine structure in the fibres which is not present in the true object. The magenta circle highlights a cross section of a tubule which appears as a lonely circle. This feature, which is incidentally unrecognisable without some form of deconvolution, appears to be in a crowded environment in the theoretical deconvolution due to amplification of noise in the neighbourhood. In the case where the bandwidth was not overestimated (Figure $4.19 \mathrm{c}$ ), this feature is correctly found to be in open space.

\subsection{Conclusions}

The challenges of STED microscopy deep inside thick specimens were studied using a new test sample capable of characterising both the depletion spot shape and the resulting fluorescent PSF at an effectively continuous range of imaging heights. The height dependence of the depletion spot distortion was measured and a method developed to counteract it using the objective correction collar. Using this method, it was shown that the depletion spot may be maintained in an optimal shape far from the cover glass.

With the correction collar adjusted to keep the 3D STED spot shape constant, PSFs were measured at heights up to $100 \mu \mathrm{m}$ and it was found that the compact PSF was also maintained until deep in the sample. PSF measurements for other imaging modes (conventional confocal, $2 \mathrm{D}$ STED and $60 \% 3 \mathrm{D}$ STED) were also demonstrated.

The measured PSFs were used for deconvolution of biological and colloidal image volumes but this was not found to show significant resolution improvement compared to deconvolution using a theoretical P SF. It was concluded that the small differences in conditions of the calibration sample and test samples were enough to negate the advantages of the measured PSF over the theoretical one. Specifically, the media had refractive indices differing by 0.028 and the scattering induced by the probe particles and cellular features was presumably not identical. By comparison to synthetic images, it was demonstrated that the theoretical PSF is more susceptible to noise amplification as it tends to 

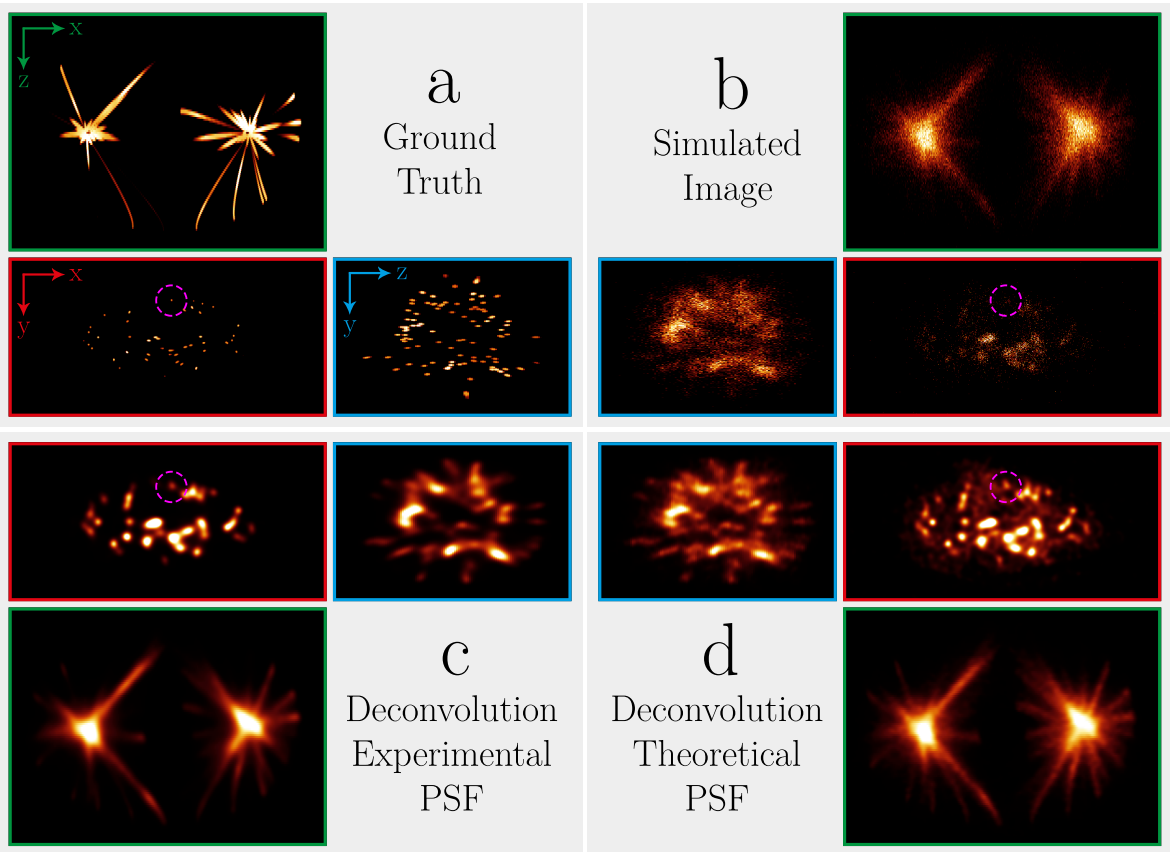

Figure 4.19: Deconvolution of a synthetic image volume using different PSFs. a) The ground truth object: representation of microtubules from Reference 151. An overview of this object is shown in Figure 4.5. b) The image simulated using the experimentally measured PSF in Figure 4.8 (3D STED, $0 \mu \mathrm{m}$ ) and Poisson noise with $\mathrm{SNR}=7$. c) Deconvolution result using the experimental (in this case, true) PSF. d) Deconvolution result using a theoretically calculated PSF under the same conditions (Figure 4.12). Comparable slices are bordered in the same colour and the magenta circles highlight a feature in the $x y$ slices to assist comparison. 
overestimate the bandwidth of the microscope. The measured PSF did did not result in spurious features this way, because the bandwidth was known to be narrower.

In conclusion, the new test sample allows a convenient method of quality control for long working distance STED microscopes. This allows the microscopist to avoid over fitting during deconvolution of deep image volumes but significant gains in resolution by deconvolution with measured STED PSFS in a well adjusted microscope remain for future work.

\subsection{Outlook}

The limitations of the test sample and method presented in this chapter lie principally in the fixed refractive index and the low signal of the fluorescent beads. The first means that the depletion beam optimisation settings and measured PSFs will only be valid for specimens with the same refractive index. It was demonstrated that even small differences in refractive index can negate any advantage of the measured PSF over a theoretical one in deconvolution. A theoretical route to extrapolate the measured PSFs to different sample media would greatly extend the applicability of the method.

The low signal which could be obtained from the small fluorescent beads before photobleaching limited the fidelity of the measured PSFs. With brighter fluorescent beads, or beads immune to bleaching such as quantum dots, sufficient signal could be obtained to capture tertiary and quaternary features of the PSF. Such PSFs would likely be able to provide significant gains through deconvolution.

One application with potential for significant gains is microscopy under more challenging conditions where a measured depth dependent confocal PSF might be the only way to achieve a meaningful deconvolution. For example, for imaging through a layer of PDMS in a microfluidic chip, a test sample like this one could be made which incorporates this layer for PSF measurement.

There are also parts of this method which could be readily automated. For example automated $\mathrm{CC}$ optimisation might yield more accurate results than those achieved 'manually' here. Sparse gold nanoparticles in an optically matched medium reflect much more brightly than the background, allowing easy identification. The automated analysis presented in Figure $4.7 \mathrm{~b}$ could be run at each height and a feedback directed to the motorised $\mathrm{CC}$ to optimise one or more of the parameters. Furthermore, with non bleaching beads, large image volumes could be captured and PSF extraction carried out without manual intervention. 


\section{Acknowledgements}

The work presented in this chapter was carried out in collaboration with Jantina Fokkema and Ernest van der Wee. Both are gratefully acknowledged for their contributions. The particles and image in Figure $4.3 \mathrm{~b}$ were made by Jantina Fokkema. The biological test sample in this chapter was kindly provided by Wilco Nijenhuis and its refractive index measured in situ by Eugene Katrukha. These are thanked for their assistance in biological microscopy, which could not have been in this chapter without their help.

\section{A Linear focal shift between excitation and depletion spots}

Here the objective lens is assumed to be a perfectly adjusted apochromat which is able to focus both $\lambda_{e}$ and $\lambda_{s}$ to the same point, located at a distance of $z_{0}^{\prime}$ in front of the cover glass as shown in Figure 4.20. When the objective is moved forwards by $\Delta z$, the focal points of $\lambda_{e}$ and $\lambda_{s}$ move forwards by $\Delta z_{e}^{\prime}$ and $\Delta z_{s}^{\prime}$ respectively. The difference between these is denoted by $\Delta F^{\prime}$. This is the mismatch which was measured in Section 4.4.1 and which is calculated here.

Examining the corresponding angles, all of the marked angles remain constant for the same two rays emerging from the objective. Therefore the factor which determines $\Delta F^{\prime}$ is the height at which each ray emerges from the cover glass, $x^{\prime}$. From basic trigonometry:

$$
\Delta z_{e}=z^{\prime}-z_{0}^{\prime}=\frac{x_{e}^{\prime}}{\tan \phi_{3}}-\frac{x_{0, e}^{\prime}}{\tan \phi_{3}},
$$

and

$$
x_{e}^{\prime}-x_{0, e}^{\prime}=x_{e}-x_{0, e}=\Delta x=\Delta z \tan \phi_{1} .
$$

$\Delta x$ is the height of the new ray as projected back onto the original lens position above the original point of emergence. Applying Snell's law at the cover glass entrance and exit:

$$
n_{1}\left(\lambda_{e}\right) \sin \phi_{1}=n_{3}\left(\lambda_{e}\right) \sin \phi_{3} .
$$

Combining the above expressions yields an expression for the focal shift of one wavelength:

$$
\Delta z_{e}^{\prime}=\frac{\Delta z \tan \phi_{1}}{\tan \phi_{3}}=\Delta z \frac{n_{3}\left(\lambda_{e}\right)}{n_{1}\left(\lambda_{e}\right)} \frac{\cos \phi_{3}}{\cos \phi_{1}}=\Delta z \frac{n_{3}\left(\lambda_{e}\right)}{n_{1}\left(\lambda_{e}\right)} \frac{\sqrt{1-\frac{n_{3}(\lambda)^{2}}{n_{1}(\lambda)^{2}} \sin ^{2} \phi_{1}}}{\cos \phi_{1}} .
$$




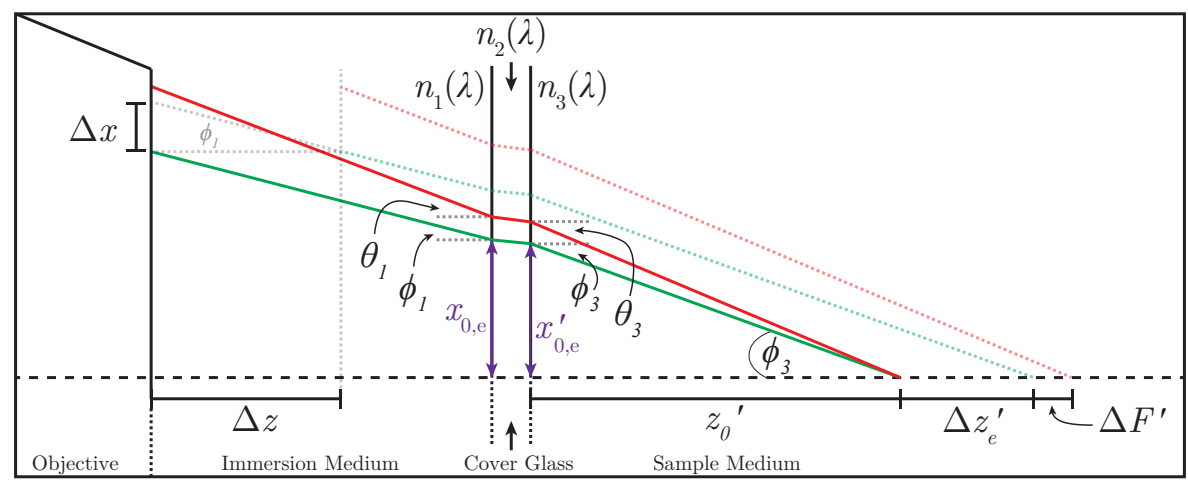

Figure 4.20: Focussing two rays of different wavelength. The solid rays in red ( S TED,$\lambda_{s}$ ) and green (excitation, $\lambda_{e}$ ) emerge from the objective such that they are focussed to the same point inside the sample. When the objective is moved forwards by distance $\Delta z$, the focal point of $\lambda_{e}$ moves forwards by $\Delta z_{e}^{\prime}$ and that of $\lambda_{s}$ moves by $\Delta z_{s}^{\prime}$. The difference between these is the mismatch which was measured in Section 4.4.1, $\Delta F^{\prime}$.

Here Snell's law was used twice: first to remove the tangents and second to reduce the dependence to a single angle. The last term on the right remains very close to unity at moderate angles and so this term is approximated to 1. This may be verified by plotting or observing that the second derivative of this term evaluated at $\phi_{1}=0$ is $1-\frac{n_{3}^{2}}{n_{1}^{2}}$, which is small when $n_{1}$ and $n_{3}$ are reasonably well matched. Using this simplification:

$$
\Delta F^{\prime}=\Delta z_{s}^{\prime}-\Delta z_{e}^{\prime} \approx \Delta z\left(\frac{n_{3}\left(\lambda_{s}\right)}{n_{1}\left(\lambda_{s}\right)}-\frac{n_{3}\left(\lambda_{e}\right)}{n_{1}\left(\lambda_{e}\right)}\right) .
$$

Thus the shift in focal point of the two beams depends on the dispersion in the immersion and sample media. This would seem to be a sufficiently small effect to be in line with the observed shift of around $1 \mathrm{~nm} / \mu \mathrm{m}$.

To test the prediction of this simplified model, the refractive indices of the two media were measured and the indices at the excitation and depletion wavelengths estimated using the two term Cauchy equation [168]:

$$
n(\lambda)=b+\frac{c}{\lambda^{2}} .
$$

The measured values and the refractive indices estimated using them are shown in Table 4.2. Substituting the calculated $n(\lambda)$ values into Equation 4.8, the 


\begin{tabular}{|l|c|c|c|c|c|c|}
\hline Medium & $n_{D}^{21}$ & $n_{F}-n_{C}$ & $b$ & $c\left(\mathrm{~nm}^{2}\right)$ & $n\left(\lambda_{e}\right)$ & $n\left(\lambda_{s}\right)$ \\
\hline 1 (immersion) & 1.4506 & $9.57 \times 10^{-3}$ & 1.4362 & 5009 & 1.4532 & 1.4477 \\
3 (sample) & 1.4230 & $7.08 \times 10^{-3}$ & 1.4123 & 3707 & 1.4249 & 1.4208 \\
\hline
\end{tabular}

Table 4.2: Refractive index and dispersion values. The refractive index $\left(n_{D}^{21}\right)$ and dispersion $\left(n_{F}-n_{C}\right)$ values were measured with an Atago $3 \mathrm{~T}$ Abbe refractometer. These were used to determine the Cauchy coefficients, $b$ and $c$ [167]. The Cauchy equation (Equation 4.9) was then used to calculate the refractive indices at the excitation and depletion wavelengths, shown in the last two columns.

displacement of the two focal spots is predicted to be $\frac{\Delta F^{\prime}}{\Delta z} \approx-9.1 \times 10^{-4} \approx$

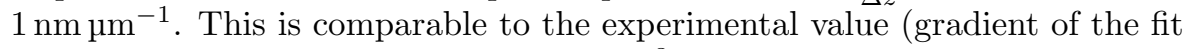
line in Figure 4.7 a) which was $-1.9 \times 10^{-3}$, a factor of two greater.

Thus this simplified model is able to reproduce the correct form and approximate magnitude of the beam misalignment with changing depth. The disparity in values likely results from the high concentration of silica in the sample, which was not present during refractive index measurement. It is concluded that the misalignment results from the different dispersive properties of the lens and sample immersion media as described by Equation 4.8. This effect could be reduced by using the same liquids for both immersions where possible. 



\title{
5 Diffusiophoresis at the single particle level
}

\begin{abstract}
Direct measurement of diffusiophoretic velocities of colloidal silica particles in electrolyte gradients is demonstrated. The gradients were generated using a microfluidic $\psi$-shaped channel and the motion of the fluorescent particles imaged with confocal microscopy. Analysis of single particle trajectories confirmed the action of diffusiophoretic transport and observations were compared to theoretical predictions using salt gradients as predicted for diffusion between laminar streams. The measured diffusiophoretic mobilities were consistently higher than those predicted by theory and this is attributed to a complication of the solute profile after investigation using a fluorescent salt. Despite this, the measured velocity profiles have shapes in close agreement with theory and it is proposed that small changes to the experimental design will reconcile the observations with predictions. With that achieved, this method would be ready to take its place in the library of standard laboratory techniques alongside microelectrophoresis.
\end{abstract}




\subsection{Introduction}

Some colloidal phenomena are easier to relate to the human experience than others. Sedimentation, for example, might be explained to the layperson by comparison to marbles sinking in a glass of water. Despite the loss of fascinating detail in such a description, the general idea may be conveyed and the results more or less predicted. Becoming more abstract, the motion of charged colloids in an electric field could be related to somebody aware of the flow of electrons from one end of a battery to the other. The explanation reached via this route would be largely incorrect but would again bestow reasonable predictive power (in that negatively charged colloids migrate towards the positively charged electrode). An example more abstract still is the subject of this chapter. The situation considered here is one in which a particle migrates neither because of nearby planets nor nearby electrodes, but because of differences in the concentration of dissolved species in the environment of the particle.

Familiarity with the way in which a drop of dissolved pigment added to water spreads out until the colour becomes uniform might suggest that the particle would also tend to move away from the region of high solute concentration. In fact, the opposite direction is more often the one which would be observed. This phenomenon is called diffusiophoresis and it involves the interplay of various microscopic effects occurring close to the particle surface. Thus it defies explanation using only macroscopic concepts and which is perhaps why this commonly occurring effect is often overlooked in favour of its more famous sibling: electrophoresis.

Diffusiophoresis was discovered with its origin immediately understood by Derjaguin et al. in 1947 for neutral solutes [169] and later for electrolytes [170]. Much of the early research into this effect was driven by its application in the manufacture of items such as rubber gloves [169-173] in a process known as coagulant latex dipping ${ }^{1}$. Alongside this application, growing recognition of the fundamental importance of diffusiophoresis led to fruitful efforts to advance the theory describing it [9, 174-180]. Recently, interest in diffusiophoresis has been reinvigorated due to its relevance to the rapidly emerging field of active colloidal matter [181].

Dukhin and Derjaguin formed the vanguard both in understanding and recognition of diffusiophoresis in the $20^{\text {th }}$ century. They wrote in $1974[174$, p. 325]:

Of great interest would be measurements of the diffusiophoretic mobility of separate particles by a procedure similar to microelectrophoresis.

A few years later, the same authors attempted just such a procedure [182]. 
This involved inserting a porous cellophane membrane into a capillary to separate an initially high electrolyte concentration region from a latex dispersion. Unfortunately, quantitative analysis was hampered by "the impossibility of establishing a spatially uniform, time-invariant gradient of concentration," as well as by gravitational convection. It must be assumed that the novelty of the current work would be minimal had modern microfabrication technology and microfluidic ideas been available in the USSR in the 1970 .

Still before the popularisation of microfluidics as it is known today, microchannels in the form of pores in fritted glass were utilised to suppress convection effects [183]. The pores were of a size that allowed passage of both the latex particles and the salt and by measuring changes in particle concentration on either side of the membrane in time, the rate of diffusiophoretic transport could be determined. Using this method, the diffusiophoretic velocities of latex particles were found to be in quantitative agreement with the theory of Reference 179 [184]. The applicability of this method was limited to particles of very thin double layer $(<6 \%$ of the particle radius, salt concentration $>\sim 10 \mathrm{~mm}$ ) by the requirement that the double layers of the particles and pore walls not interact, slowing particle flux.

In another 1980 s work of proto-microfluidics, diffusiophoresis was quantitatively measured in both electrolytes [186] and non-electrolytes [187]. The procedure in this case was to measure the banding in colloidal concentration induced by gradients in a stop-flow cell.

The broad potential of microfluidics was realised around the turn of the century and its use was accelerated by the fast prototyping technique of soft lithography [188]. This facilitated the seminal work of Abécassis et al. in which a $\psi$-shaped channel was used as an elegant way to study diffusiophoresis of colloids in many concentration gradients in a single experiment [189, 190]. By introducing coflowing laminar streams with differing solute concentrations, diffusion between the streams provided a different transverse concentration profile at each downstream position. In this system, the displacement from the junction in the flow direction corresponds to the diffusion time, so the concentration profiles are described by well-defined diffusion equations. This system was used to demonstrate diffusiophoretic spreading and focussing of silica particles. The resulting particle concentration profiles were used to determine the diffusiophoretic mobility of the particles in various electrolytes.

The aforementioned method is a powerful one but has the drawback that

\footnotetext{
${ }^{1}$ This important industrial process is variously known as coagulant dipping, coacervate dipping or ionic deposition. It involves dipping a mould coated in a coagulant (usually a salt) into a latex dispersion. The coagulant dissolves to create a salt gradient which draws the latex particles to the surface of the mould by diffusiophoresis. The particles aggregate at the surface of the mould, forming the polymer film in the desired shape [185].
} 


\section{Diffusiophoresis at the single particle level}

the particle concentration which is measured is an average across the full height of the channel and thus requires complicated manipulation to account for the Poiseuille flow velocity profile. Furthermore, the use of the width of the colloidal band to measure the diffusiophoretic transport limits the method to measuring a single diffusiophoretic velocity across the channel.

Perhaps the closest approach to the Dukhin/Derjaguin dream of microdiffusiophoresis reported to date is the innovative use of microfluidics reported in References 191 and 192. Therein, membranes porous to ions but not particles were incorporated into the side walls of a stop-flow channel. This allowed establishment of well defined electrolyte gradients across the channel in which polystyrene particles were dispersed. Particle motion was measured using micro particle image velocimetry ( $\mu$ P IV ), a technique which measures the velocity field in a micron-sized area from the cross correlation of successive microscopic video frames [193]. Thus the resolution of diffusiophoretic measurement was increased from that of ensemble concentration profiles to that of small groups of particles [194].

In this chapter, the resolution will be further increased to that of single colloidal silica particles by building upon our recent work [195]. The $\psi$ channel geometry of Reference 190 was chosen over the membrane cell both for its ease of fabrication and elegance of creating a broad range of concentration gradients in a single experimental run. Previous work in such channels [189, 190, 196] is extended by measuring the diffusiophoretic velocities not from averaged concentration profiles, but from the trajectories of single particles. This allows a direct observation of the diffusiophoretic effect, and a way to readily quantify it. Furthermore, with knowledge of where every particles is in the concentration gradient, the diffusiophoretic mobility may be measured as a function of the electrolyte concentration.

After a discussion of the relevant theory and methods in the following sections, independent characterisation of the zeta potential is presented. This measurement facilitates a quantitative comparison with the theory of diffusiophoresis. The results of the main diffusiophoresis experiments are then presented along with comparisons to the established theoretical predictions. While high resolution velocity profiles were measured, it was not possible to quantitatively reconcile the experiments with the theory. Therefore, an investigation into the most likely source of inconsistency, the solute concentration profile, may be found in Section 5.4.4. Finally, the chapter is concluded and suggestions made for future work. 


\subsection{Theory}

\subsubsection{Diffusiophoresis}

Consider a charged spherical particle suspended in a homogeneous aqueous solution of electrolyte. Adjacent to the particle surface is the electrical double layer, which contains an excess of counter ions with opposite sign to that of the surface charge. Due to the excess of entities attracted to the particle surface, the osmotic pressure inside the double layer is higher than that in the bulk solution [197]. This pressure decays exponentially with radial distance from the particle, in a way that depends on the screening of the particle charge. At higher salt concentration, the surface charge is screened more effectively, resulting in a higher osmotic pressure double layer of smaller extent, and vice versa.

Now consider that the electrolyte concentration is no longer homogeneous. In this case, the double layer rearranges so as to maintain equilibrium with the electrolyte adjacent to the outer extent of the double layer [174]. With the double layer thus polarised, the osmotic pressure within is higher at the high salt side of the particle. The osmotic pressure gradient along the surface of the particle induces a slip velocity tangential to the particle surface, towards the lower salt side of the particle. The liquid in the interfacial region is in contact with both the particle and the bulk solution. Since the latter is at rest in the laboratory frame, the particle is observed to move up the salt gradient. This effect is known as chemiphoresis. Here, the discussion has been focussed on electrolyte solutions ${ }^{2}$ because of an additional effect occurring in concentration gradients of salts: electrophoresis.

While the diffusion coefficients of the two ions in a monovalent electrolyte solution may have similar magnitude (as for $\mathrm{KCl}$ ), this is not in general the case. For example, in aqueous $\mathrm{NaCl}$ solution, the chloride anion diffuses more rapidly than the cation and in $\mathrm{HCl}$ solution, the opposite is true. The result is that, in a gradient, a macroscopic electric field (the diffusion potential) arises which acts to slow the faster ion and speed up the slower one. This electric field also acts upon colloidal particles in the gradient, inducing electrophoresis in the same way as if the electric field had been applied externally. The field direction depends on the relative diffusivities of the electrolyte ions, characterised (for

\footnotetext{
${ }^{2} \mathrm{~A}$ similar discussion may also be applied to gradients of neutral solutes, but with soluteparticle attractions (or repulsions) causing the osmotic pressure differences in the interfacial layer. In the repulsive case, chemiphoresis drives the particle down a neutral solute gradient [177].
} 
$z: z$ electrolytes) by

$$
\beta=\frac{D_{+}-D_{-}}{D_{+}+D_{-}} .
$$

Thus the two effects may act in harmony to move the particle, or may compete to drive it in opposite directions. Prediction of the resulting diffusiophoretic velocity requires accounting for both effects and various efforts to do so have been made in the surface science community. In this chapter, the 1984 theory of Prieve et al. [178] is used. While more recent developments by the same authors [179] and others [198] are more exact, the method in Reference 178 is conveniently implemented and will be shown to agree with more accurate predictions in the region of parameter space in which the work of this chapter is situated.

The approach taken was to approximate the in-gradient behaviour of the thin but finite double layer as a perturbation to its equilibrium structure. The thickness of the double layer in relation to the particle may be characterised by $\lambda=(\kappa a)^{-1}$ with $a$ the particle radius and $\kappa$ the inverse Debye length in $\mathrm{nm}^{-1}[199]$ :

$$
\kappa=3.288 \sqrt{I}
$$

in water at $25^{\circ} \mathrm{C}$ with ionic strength $I=1 / 2 \sum c_{i} z_{i}^{2}\left(c_{i}\right.$ is the molar concentration of each ionic species with charge $z_{i}$ ). In the $\lambda \rightarrow 0$ limit, the double layer is so thin compared to the particle curvature that the slip velocity is that of a flat wall, for which an exact solution is known [170, 174, 178].

The total diffusiophoretic velocity $v_{d p}$ results from the combination of chemiphoretic and electrophoretic effects. In the case of a weak ${ }^{3}$ electrolyte gradient [178]:

$$
v_{d p}=v_{c}+v_{e}
$$

$v_{c}$ is the chemiphoretic velocity in the presence of a concentration gradient of a symmetric electrolyte with $\beta=1 . v_{e}$ is the electrophoretic velocity, which would be observed if the diffusion potential were replaced by an equivalent external electric field. By treating the case of a thin double layer as a perturbation to the $\lambda=0$ case (for which analytical solutions are available), it was found that $[178,179]$ :

$$
v_{d p} \approx v_{c 0}\left[1+G_{c 0} \lambda+\mathcal{O}\left(\lambda^{2}\right)\right]+v_{e 0}\left[1+G_{e 0} \lambda+\mathcal{O}\left(\lambda^{2}\right)\right]
$$

\footnotetext{
${ }^{3}$ The requirement is that the diffusiophoretic velocity must be much slower than the diffusion of the electrolyte. If this is not the case, Equation 5.3 becomes nonlinear [178].
} 
with $v_{c 0}$ and $v_{e 0}$ being the exact solutions for $\lambda=0[170,174,176]$ :

$$
\begin{aligned}
& v_{c 0}=\frac{k T}{2 \pi \eta l_{B}}\left[-\ln \left(1-\gamma^{2}\right)\right] \nabla \ln c, \\
& v_{e 0}=\frac{k T}{4 \pi \eta l_{B}} \beta \bar{\zeta} \nabla \ln c .
\end{aligned}
$$

The Bjerrum length, $l_{B}=e^{2} / 4 \pi \epsilon_{0} \epsilon_{r} k T \approx 0.7 \mathrm{~nm}$ in water at room temperature and $\eta$ is the solvent viscosity. The non-dimensional zeta potential is denoted by $\bar{\zeta}=z e \zeta / k T$ and $\gamma=\tanh (\bar{\zeta} / 4)$. $e$ is the elementary charge, $\zeta$ is the dimensional zeta potential, $k$ is Boltzmann's constant and $T$ is the absolute temperature. Equation 5.5 is analogous to diffusiophoresis in a neutral solute gradient $[170,177]$ and approaches this case in the limit $\lambda \rightarrow 0$ and $\bar{\zeta}<<1$ [178]. Equation 5.6 reduces to Smulochowski's equation for electrophoresis in the diffusion potential in the small $\lambda$ limit [200]. The modifying $G$ factors are defined:

$$
\begin{aligned}
G_{c} & =-\frac{F_{0}+P e\left(F_{2}+\beta F_{3}\right)}{4 \ln \left(1-\gamma^{2}\right)}, \\
G_{e} & =\frac{F_{1}+P e\left(\beta F_{4}+F_{5}\right)}{2 \bar{\zeta}},
\end{aligned}
$$

with

$$
P e=\frac{k T}{8 \pi \eta D_{s} l_{B}},
$$

a Péclet number. Diffusion of the electrolyte is characterised by the coupled diffusion coefficient given by the Nernst-Hartley equation: $D_{s}=2 D_{+} D_{-} /\left(D_{+}+\right.$ $\left.D_{-}\right)$with $D_{ \pm}$the diffusion coefficients of the separate ions [201]. The $F_{n}(\bar{\zeta})$ functions characterise the perturbation of the thin double layer away from its equilibrium structure and are tabulated in Appendix B of Reference 178. In order to reasonably neglect the $\mathcal{O}\left(\lambda^{2}\right)$ terms in Equation $5.4,|G \lambda|$ should be small, limiting the applicability of this relation to very small $\bar{\zeta}$. In Reference 178 , a Padé approximant was used to extend the range of validity to larger $\zeta$ :

$$
v_{d p} \approx \frac{v_{c 0}}{1-G_{c} \lambda}+\frac{v_{e 0}}{1-G_{e} \lambda} .
$$

Equation 5.10 provides a convenient way to approximately predict the diffusiophoretic velocity for a system and it is used for the theoretical predictions in this chapter. Figure 5.1 shows the predicted diffusiophoretic mobility for particles with a range of $\bar{\zeta}$ at various values of $\lambda$ in a $\mathrm{NaCl}$ gradient in order to compare with the more exact predictions from Reference 179. Colloidal silica 

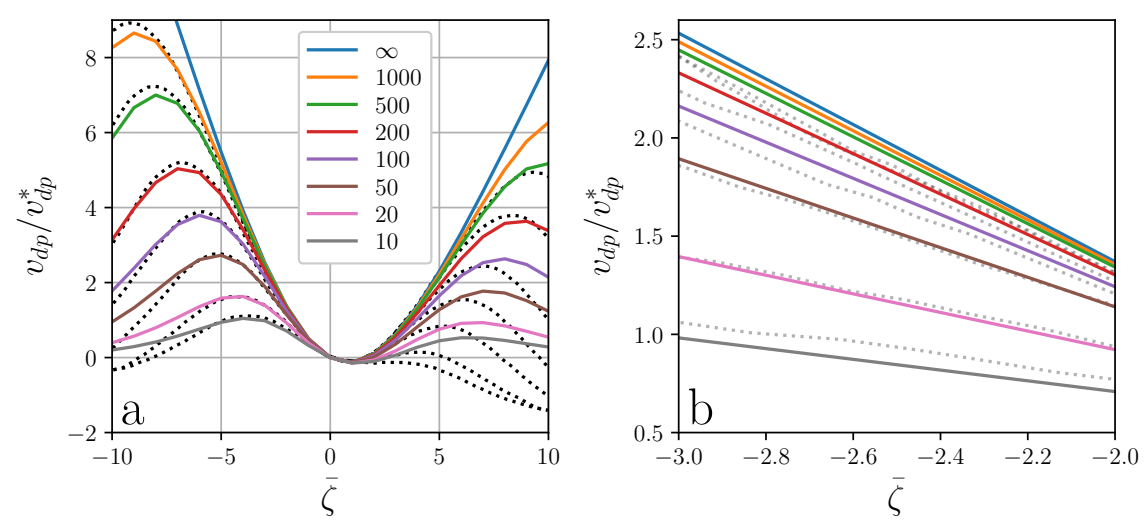

Figure 5.1: Theoretical prediction of the diffusiophoretic velocity in a $\mathrm{NaCl}$ gradient. a) Comparison of the predictions from Equation 5.10 (solid lines) with the more exact results from Figure 9a in Reference 179 (dotted lines). The legend indicates the $\kappa a=\lambda^{-1}$ value of each curve. b) The $\bar{\zeta}$ region relevant to this work at larger scale. The dotted lines are less reliable here because they were traced from a graph at scale similar to (a). $v_{d p}^{*}=k T\left|\nabla \ln c_{s}\right| / 6 \pi \eta l_{B} \sim$

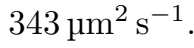

generally has $\bar{\zeta}$ in the range $\sim[-1,-3]$ and the agreement between the two approximations in this region is seen to be excellent. The largest deviations occur at $\bar{\zeta}>1$ which is not encountered in the experiments in this chapter. The analytical solution at $\kappa a=\infty$ (differentially thin double layer) is also shown.

Noting the gradient in chemical potential as a common factor in Equations 5.4, 5.5 and 5.6, it seems convenient to define a diffusiophoretic mobility, $D_{d p}$ such that

$$
v_{d p}=D_{d p} \nabla \ln c_{s}=D_{d p} \frac{1}{c_{s}} \nabla c_{s},
$$

in analogy to the electrophoretic mobility, $\mu_{E}$. However, this step should be taken with some care lest a crucial difference between these phoretic effects be lost in the beguiling simplicity of Equation 5.11. While both are affected by the extent of the double layer ( $\lambda$ in Equation 5.10 and $\kappa a$ in Equation 5.13), only in diffusiophoresis is the value of $\kappa a$ liable to change due to the transport. As the particle moves to a region of higher or lower electrolyte concentration, the double layer will compress or expand. Thus $D_{d p}$ depends not only on 
the properties of the particle and salt, but also the instantaneous position of the particle within the electrolyte gradient. The $\kappa a$ dependence of $D_{d p}$ is frequently overlooked without justification in the more recent experimental literature, with the diffusiophoretic mobility being assumed to be given by the sum of Equations 5.5 and 5.6 and therefore independent of particle size and ionic strength [189-191, 197].

\subsubsection{Zeta potential measurement with electrophoresis}

A charged colloidal particle in an electric field is induced to move with velocity, $\mathbf{v}$, proportional to the field strength, E:

$$
\mathbf{v}=\mu_{E} \mathbf{E} .
$$

The constant of proportionality, $\mu_{E}$, is the electrophoretic mobility. A good approximation of this mobility is given by the Henry formula [200]:

$$
\mu_{E}=\frac{2 \epsilon_{r} \epsilon_{0} \zeta f_{1}(\kappa a)}{3 \eta}
$$

where $\eta$ and $\epsilon_{r}$ are the viscosity and relative permittivity of the solvent, $\epsilon_{0}$ the permittivity of free space and $\zeta$ the zeta potential. Thus by measuring the velocity of particles in an imposed electric field, the zeta potential may be obtained. $f_{1}(\kappa a)$ is a monotonically increasing function which incorporates the dependence of the mobility on the double layer thickness and particle size. It connects the two limiting cases of infinitely thin double layer (the Smoluchowski limit, $\left.\kappa a=\infty, f_{1}(\kappa a)=3 / 2\right)$ and infinitely thick double layer (the Hückel limit, $\kappa a=0, f_{1}(\kappa a)=1$ ). In this work, the Ohshima form of $f_{1}(\kappa a)$ is used [200, 202]:

$$
f_{1}(\kappa a)=1+\frac{1}{2(1+\delta / \kappa a)^{3}},
$$

with

$$
\delta=\frac{2.5}{1+2 \exp (-\kappa a)} .
$$

This approximates well the behaviour of $\mu_{E}(\zeta)$ for low $\zeta$ and extreme values of $\kappa a$. For the moderate $\zeta$ and $\kappa a$ values encountered in this chapter, Equation 5.13 slightly overestimates $\mu_{E}$ and thus the derived zeta potentials are slightly low. While more exact values of $\mu_{E}$ may be obtained by numerically solving the governing differential equations in the electrolyte [203], the precision of the electrophoretic mobility measurement in this chapter did not justify this step. 


\subsubsection{Salt gradient evolution along the channel}

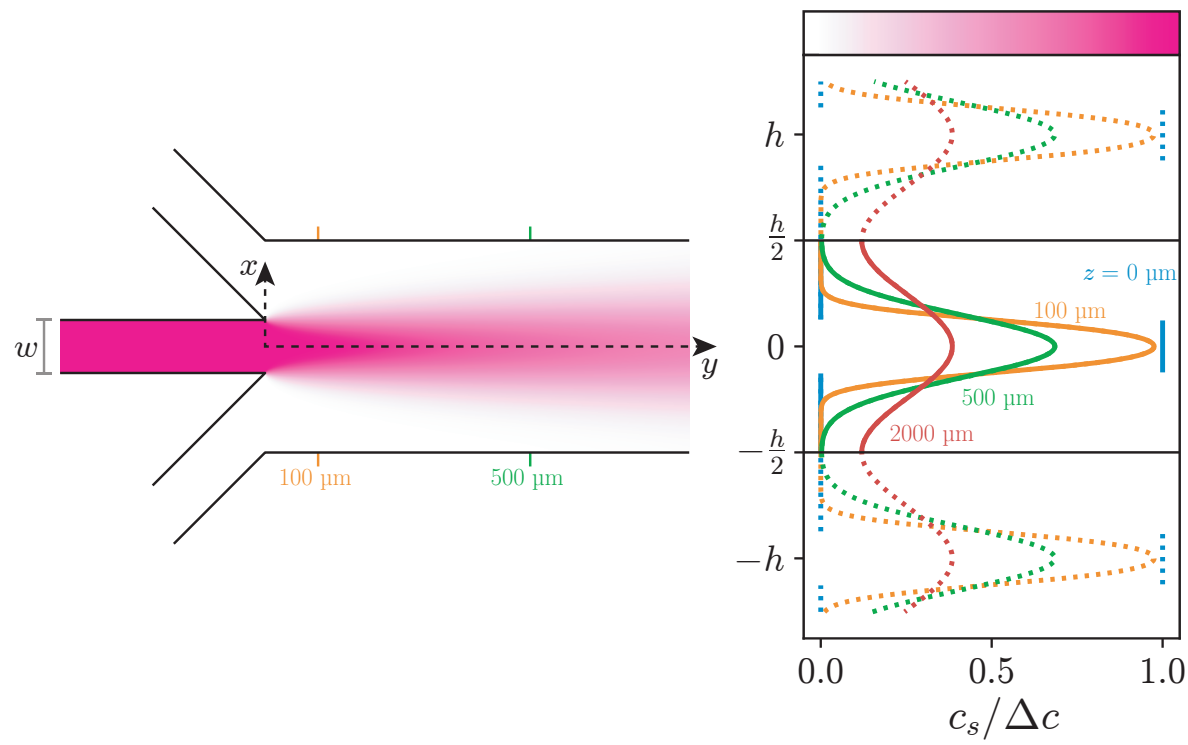

Figure 5.2: Solute diffusion in a microfluidic $\psi$ channel calculated using Equation 5.18. On the left is depicted a cross section of the device with a magenta solute in the central channel and pure solvent in the coflowing outer channels. The origin of the coordinate system is at the centre of the inlet in the plane of confluence and the $z$ axis points into the page. The graph on the right shows the concentration profile along $x$ at various $y$ positions, turned on its side to share the $x$ axis. The solid coloured lines show the solute concentrations within the channel while the broken lines show the first (of infinite) reflection images used to satisfy the boundary conditions. The colourbar relates the concentration axis to the colour in the diagram. The parameters in the calculation are as follows: $D_{s}=430 \mu^{2} \mathrm{~s}^{-1}, \bar{v}=174 \mu \mathrm{ms}^{-1}$ (flow rate $15 \mu \mathrm{L} \mathrm{h}^{-1}$ ), $h=400 \mu \mathrm{m}, w=\frac{h}{4}, c_{0}=0$ and $\Delta c=1$. The markers at the channel sides indicate $y=100$ and $500 \mu \mathrm{m}$, two of the sample points in the concentration graph.

The steady state concentration of a solute, $c_{s}(x, y, z)$, introduced into a channel as depicted in Figure 5.2 obeys a convection-diffusion equation [190]:

$$
v_{y} \frac{\partial}{\partial y} c_{s}(x, y, z)=D_{s} \nabla^{2} c_{s}(x, y, z)
$$


Here, $v_{y}$ is the fluid flow velocity which is assumed to be well described by Hele-Shaw flow such that $v_{y}(z)=\frac{3}{2} \bar{v}\left[1-\left(2 z / z_{0}\right)^{2}\right]$, with $\bar{v}$ the mean flow velocity and $z_{0}$ the channel depth in the $z$ direction [204]. $\nabla^{2}$ is the Laplacian operator and $D_{s}$ is the solute diffusion coefficient.

Since the depth of the channel, $z_{0}$, is small relative to its width, $h$, rapid diffusion in the $z$ direction may be assumed such that the $z$ dependence of $c_{s}$ may be neglected. This has been shown to be valid for $y \gtrsim \frac{1}{10} \frac{\bar{v}}{D_{s}}\left(\frac{z_{0}}{2}\right)^{2}[205]$. Furthermore, since the advective transport is much faster than diffusive transport, diffusion along $y$ may also be neglected. Thus Equation 5.16 may be simplified to [190]:

$$
\bar{v} \frac{\partial}{\partial y} c_{s}(x, y)=D_{s} \frac{\partial^{2}}{\partial x^{2}} c_{s}(x, y) .
$$

This may be solved by imposing no-flux boundary conditions at the channel walls $\frac{\partial}{\partial x} c_{s}(x= \pm h / 2, y)=0$ and a central stationary point due to symmetry $\frac{\partial}{\partial x} c_{s}(x=0, y)=0$. The 'initial' concentration profile at $y=0$ may be constructed from superimposed step functions [206]:

$$
c_{s}(x, y=0)=c_{0}+\Delta c\left[\mathrm{H}\left(x+\frac{w}{2}\right)-\mathrm{H}\left(x-\frac{w}{2}\right)\right],
$$

where $\mathrm{H}$ is the Heaviside step function, $w$ is the middle channel width (as in Figure 5.2), $c_{0}$ is the solute concentration in the outer channel and $\Delta c$ is the concentration difference between the inner and outer channels. $\Delta c>0$ corresponds to outward solute spreading and $\Delta c<0$ corresponds to inward spreading.

A solution to the diffusion equation for an initial superposition of step functions is a superposition of error functions of the form $\operatorname{erf}(x)=\frac{2}{\sqrt{\pi}} \int_{0}^{x} \exp \left(-u^{2}\right) d u$. Finally, to satisfy the no-flux boundary condition at the walls, mirror images of the concentration profile reflected in the walls are placed as shown in Figure 5.2. It may be seen that the symmetry of this arrangement forces the concentration profile to be stationary at the walls [207]. To keep the images in reflection of reality, they also require further images, meaning that the solution takes the form of two infinite sums of error functions:

$$
c_{s}(x, y)=c_{0}+\frac{\Delta c}{2}\left[\sum_{k=-\infty}^{\infty} \operatorname{erf}\left(\frac{x+\frac{w}{2}+k h}{2 \sqrt{\frac{D_{s} y}{\bar{v}}}}\right)-\sum_{k=-\infty}^{\infty} \operatorname{erf}\left(\frac{x-\frac{w}{2}+k h}{2 \sqrt{\frac{D_{s} y}{\bar{v}}}}\right)\right] .
$$


In the context of this work, the gradient in the solute concentration is also important:

$$
\frac{\partial c_{s}}{\partial x}=\frac{\Delta c}{2 \sqrt{\frac{\pi D_{s} y}{\bar{v}}}}\left[\sum_{k=-\infty}^{\infty} \exp -\left(\frac{x+\frac{w}{2}+k h}{2 \sqrt{\frac{D_{s} y}{\bar{v}}}}\right)^{2}-\sum_{k=-\infty}^{\infty} \exp -\left(\frac{x-\frac{w}{2}+k h}{2 \sqrt{\frac{D_{s} y}{v}}}\right)^{2}\right]
$$

\subsection{Methods}

\subsubsection{Particle synthesis and characterisation}

$\varnothing 460 \mathrm{~nm}$ (polydispersity 10\%) spherical silica particles with a $400 \mathrm{~nm}$ rhodaminedyed core inside an undyed shell were made using a well established modified Stöber method [26, 27, 40]. Particle diameters were measured from electron micrographs (Phenom Prox SEM, FEI). The particles were washed into ethanol by centrifugation after synthesis and transferred into deionised water (Milli-Q) shortly before use in experiments. The particles have gravitational length approximately $14 \mu \mathrm{m}$, corresponding to a sedimentation velocity around $80 \mathrm{nms}^{-1}$.

The electrophoretic mobilities of the particles in aqueous $\mathrm{LiCl}$ solutions was measured using laser Doppler velocimetry in a folded capillary cell (Zetasizer Nano, Malvern). Dispersions were prepared with identical particle concentrations (volume fraction $\phi \sim 3 \times 10^{-4}$ ) and $\mathrm{LiCl}$ concentrations between 0 and $10 \mathrm{~mm}$. The conductivities of the dispersions measured during the same measurement are also reported.

\subsubsection{Microfluidic Experiments}

The geometry of the device is depicted in Figure 5.2, with two inlets (outer two channels connected to the same one) and one outlet to waste. 3 channels of width $w=100 \mu \mathrm{m}$ merge into a channel of width $h=400 \mu \mathrm{m}$. An inverse master mould with height $60 \mu \mathrm{m}$ was fabricated in S U 8 on silicon using standard soft lithography techniques [188]. From this, a PDMS device was made and entry holes bored using a $1.5 \mathrm{~mm}$ biopsy punch (Miltex). The device was then bonded to a \#1.5 borosilicate cover glass using air plasma. Liquids were introduced into the device via $1.6 \mathrm{~mm}$ (outer diameter) P TFE tubing connected to glass syringes (ILS) mounted on a Cetoni Nemesys 290N syringe pump. 
For diffusiophoresis experiments, dispersions of the particles in deionised water were prepared either without added electrolyte or with salt ( $\mathrm{LiCl}$ or $\mathrm{NaCl}$ ) added to a concentration of $10 \mathrm{~mm}$.

To examine the ensemble averaged behaviour (Figure 5.4a-d), the particle concentration was $\phi \sim 4 \times 10^{-4}$ and dispersions were input only to the inner or outer channels. In the other channel(s), either deionised water or a $10 \mathrm{~mm}$ solution of $\mathrm{LiCl}$ flowed. In Figures $5.4 \mathrm{a}$ and $\mathrm{b}$, the flow rate in the large channel was $15 \mathrm{LL} \mathrm{h}^{-1}$, while in c,d it was increased to $60 \mu \mathrm{L} \mathrm{h}^{-1}$.

In single-particle experiments, particles were in both inlets. Dispersions $\left(\phi \sim 3 \times 10^{-5}\right)$ differing only in salt concentration were pumped into the device, either with the salty dispersion in the inner channel or in both of the outer channels. The syringe feeding the two outer channels pushed liquid at $10 \mu \mathrm{Lh}^{-1}$, while the one feeding the single central channel pushed at $5 \mathrm{LL} \mathrm{h}^{-1}$. A third syringe partially filled with deionised water was connected to the end of the main channel and set to pull liquid at $15 \mu \mathrm{L} \mathrm{h}^{-1}$ to stabilise flow in the device.

\subsubsection{Microscopy and image analysis}

All images were captured using a Leica TCS SP 8 inverted confocal microscope. For excitation, a $488 \mathrm{~nm}$ or $543 \mathrm{~nm}$ laser line was used. Fluorescence emission was detected by a hybrid detector.

\section{Ensemble measurements}

$450 \times 450 \mu \mathrm{m}$ images were captured using a 20x/0.70 dry (HC PL A P O, Leica) objective lens. The imaging time was $82 \mathrm{~s}(200 \mathrm{~Hz}$ scanning of $512 \times 512$ pixel raster accumulating each line 4 times and averaging the image 8 times). The calculated optical section was $\sim 2.5 \mu \mathrm{m}$.

Each frame contained the full width of the channel and by taking 5 frames separated along $y$ by $400 \mu \mathrm{m}$, the first $2 \mathrm{~mm}$ of the channel was imaged.

\section{Single particle measurements}

To resolve single particles, a $63 \times / 1.30$ glyc (HC PL APO CS 2 , Leica) objective lens was used with $85 \mathrm{wt} . \%$ glycerol/water mixture as immersion. 2000 video frames with $x y$ extent $50 \times 50 \mu \mathrm{m}$ were captured at 50 frames per second $(12 \mathrm{kHz}$ bidirectional scanning of $128 \times 128$ pixel raster, averaging each line 3 times). With axes as depicted in Figure 5.2, the scan direction was aligned with the $y$ axis and all videos were captured at the $z$ centre of the channel. The calculated optical section was $0.72 \mu \mathrm{m}$. 
At each of the sampled $y$ positions along the channel, 8 such videos were captured at $50 \mu \mathrm{m}$ intervals along $x$ to span the $400 \mu \mathrm{m}$ width of the channel.

The particle locations in these videos were fitted and linked into trajectories using Trackpy [64, 65]. To avoid spurious links, the average particle velocity from a first short tracking run was used as a predictor for the main linking routine. Tracking results were verified by overlaying trajectories on the images and checking for false links.

The trajectories were shifted to place the channel centre at the origin and then rotated to counteract the small misalignment between the channel and the $y$ axis. The 8 sets of trajectories were then translated by their relative $x y$ stage positions and combined to a full set of trajectories spanning the channel. The $v_{x}$ values were divided into 18 equal length bins along $x$ and the mean taken to be the diffusiophoretic velocity at the bin position.

\subsubsection{Fluorescent dye diffusion}

To investigate the solute diffusion behaviour in the channel, aqueous sodium fluorescein (Sigma Aldrich, F 6377 ) solution (1.7 mm) was pumped to one input and deionised water to the other (Figure 5.8). This dye contains two dissociable sodium ions but only one is dissociated at the slightly acidic $\mathrm{pH}$ of deionised water [208]. The total flow rate in the channel was $15 \mathrm{LL} \mathrm{h}^{-1}$. By measuring the fluorescence intensity (confocal imaging, parameters similar to those in 'Ensemble measurements' in the previous section), the solute concentration was approximated.

Unfortunately, the concentration of the dye solution was mistakenly made to be much higher than that at which self-quenching becomes significant [208]. This means that the proportional dependence of fluorescence intensity on dye concentration was limited to the diffuse edges of the dye front.

\subsection{Results and Discussion}

\subsubsection{Zeta Potential}

From the discussion in Section 5.2.1, it is clear that the zeta potential is a crucial parameter in the theoretical prediction of diffusiophoretic velocities. The electrophoretic mobility (in homogeneous electrolyte solution) can readily be measured and the zeta potential inferred using Equation 5.13. The results of doing so for the silica particles used in this chapter are plotted in Figure 5.3.

To ensure that the particles behave as intended at all points along the salt gradient, $\mu_{E}$ was measured at various concentrations of $\mathrm{LiCl}$ across the 

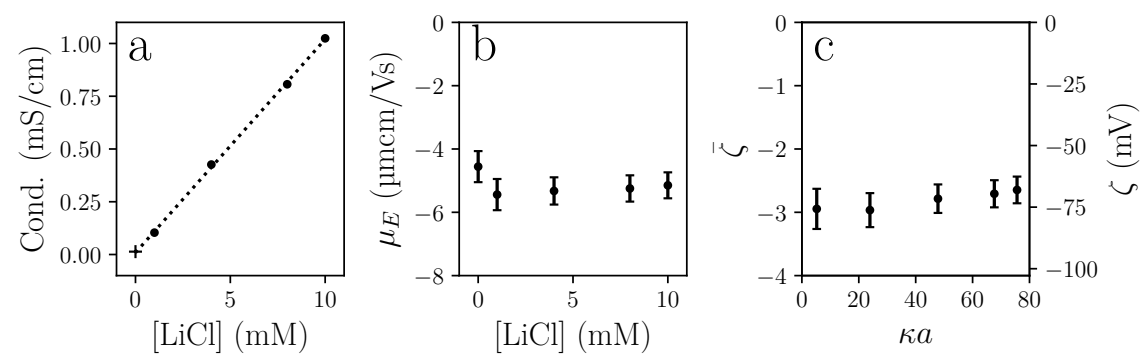

Figure 5.3: Conductivity and zeta potential measurements. a) Conductivity of suspensions as a function of $\mathrm{LiCl}$ salt concentration. The dotted line is a linear fit to the four nonzero-salt points with gradient $0.10 \mathrm{mS} \mathrm{cm}^{-1} \mathrm{mM}^{-1}$ and intercept $8.8 \times 10^{-3} \mathrm{mS} \mathrm{cm}^{-1}$. The conductivity of the dispersion without added $\mathrm{LiCl}$ is marked with the '+' symbol. b) The electrophoretic mobility, $\mu_{E}$, measured as a function of the salt concentration with a Zetasizer. c) The dimensionless zeta potential, $\bar{\zeta}$, computed from the mobilities in (b) using Equation 5.13. The right axis shows the dimensional zeta potential, $\zeta$. The abscissa was converted to $\kappa a$ using Equation 5.2. The ionic strength in the no-added-salt case was estimated by computing the $\mathrm{LiCl}$ concentration corresponding to the measured conductivity using the fit in (a). The error bars in (b) and (c) indicate one standard deviation in the mobility measurement.

concentration range used in the gradient experiments. Figure 5.3a shows that the conductivity of the dispersion increased linearly with salt concentration as expected. The value of the conductivity without added salt $\left(14 \mu \mathrm{S} \mathrm{cm}^{-1}\right)$ was used to estimate an equivalent salt concentration due to trace ions and dissociated silanol groups as $25 \mu \mathrm{M}$.

Figure $5.3 \mathrm{c}$ shows that the zeta potential was measured to be approximately constant at a $\bar{\zeta}$ value around $-2.9(75 \mathrm{mV})$ across the electrolyte concentration range.

\subsubsection{Diffusiophoresis of the ensemble}

While the aim of this work is to observe single particle diffusionphoresis, magnifying directly to that level would risk being unable to see the forest for the trees. There, every drift or fluctuation might be misidentified as the soughtafter phoretic transport. Therefore in this section, the average behaviour of 
many particles across the whole channel is examined. In this way, any conclusions from looking at individual trees in the following sections gain credibility by being consistent with the behaviour of the forest.

To do this, an experiment of the type used in Reference 190 was qualitatively carried out. This involved observing the evolution of the particle concentration in the channel after particles were introduced in either the inner or outer inlets only. From previous experimental studies [186, 190], as well as a small extension of the theory discussed in Section 5.2.1 to many particles, the profiles characteristic of diffusiophoresis are known and may be used to confirm its action.

Figure $5.4 \mathrm{a}-\mathrm{d}$ shows the results of these experiments, specifically with silica particles in $\mathrm{LiCl}$ gradients. Panels a and b show the results when the particles and added salt enter the main channel through different inlets. With the particles expected to move up the salt gradient, the spreading of the colloidal intensity profile might be attributed to diffusion. However, two features are visible evidence for superdiffusive transport. First is the non-monotonous behaviour of the concentration profile transverse to the flow. In Figure 5.4a, three bands of higher intensity are visible at the centre and sides of the profile. This occurs because the particles are driven outwards by diffusiophoresis, except at $x=0$ in the centre, where the electrolyte gradient passes through 0 . If the colloids were simply diffusing, the cross sectional profile would be expected to be high in the middle and decrease monotonously to low at the edges, as in the solute diffusion depicted in Figure 5.2. The same effect is visible in Figure 5.4 b, where the region adjacent to the colloid free zone has high intensity.

The second indicator of diffusiophoresis is the timescale of transport. For the flow rate in Figures $5.4 \mathrm{a}$ and b, the time to flow from nozzle to the end of the image was $12.3 \mathrm{~s}$, after which time the root-mean-square displacement of the freely diffusing particles would be around $5 \mu \mathrm{m}$. The migration distance of the particle front is seen to be considerably greater ${ }^{4}$.

In Figures $5.4 \mathrm{c}$ and $\mathrm{d}$, the diffusiophoretic contribution is more obvious. Now that the regions of high salt and colloid concentrations are initially the same, diffusiophoresis drives the particles in the opposite direction to diffusion, so the particles are observed to focus into more compact streams than the initial one.

Finally, the observation of both spreading and focussing of the inner channel, nonspecific hydrodynamic effects from the nozzle shape or flow rates may be ruled out as dominating factors. Thus it is concluded that diffusiophoresis is taking place and it should be possible to measure it by following the motion

${ }^{4}$ For scale: the linewidth of the text indicating the scale bar length in Figure $5.4 \mathrm{~d}$ corresponds to $5 \mu \mathrm{m}$ in the vertical direction 

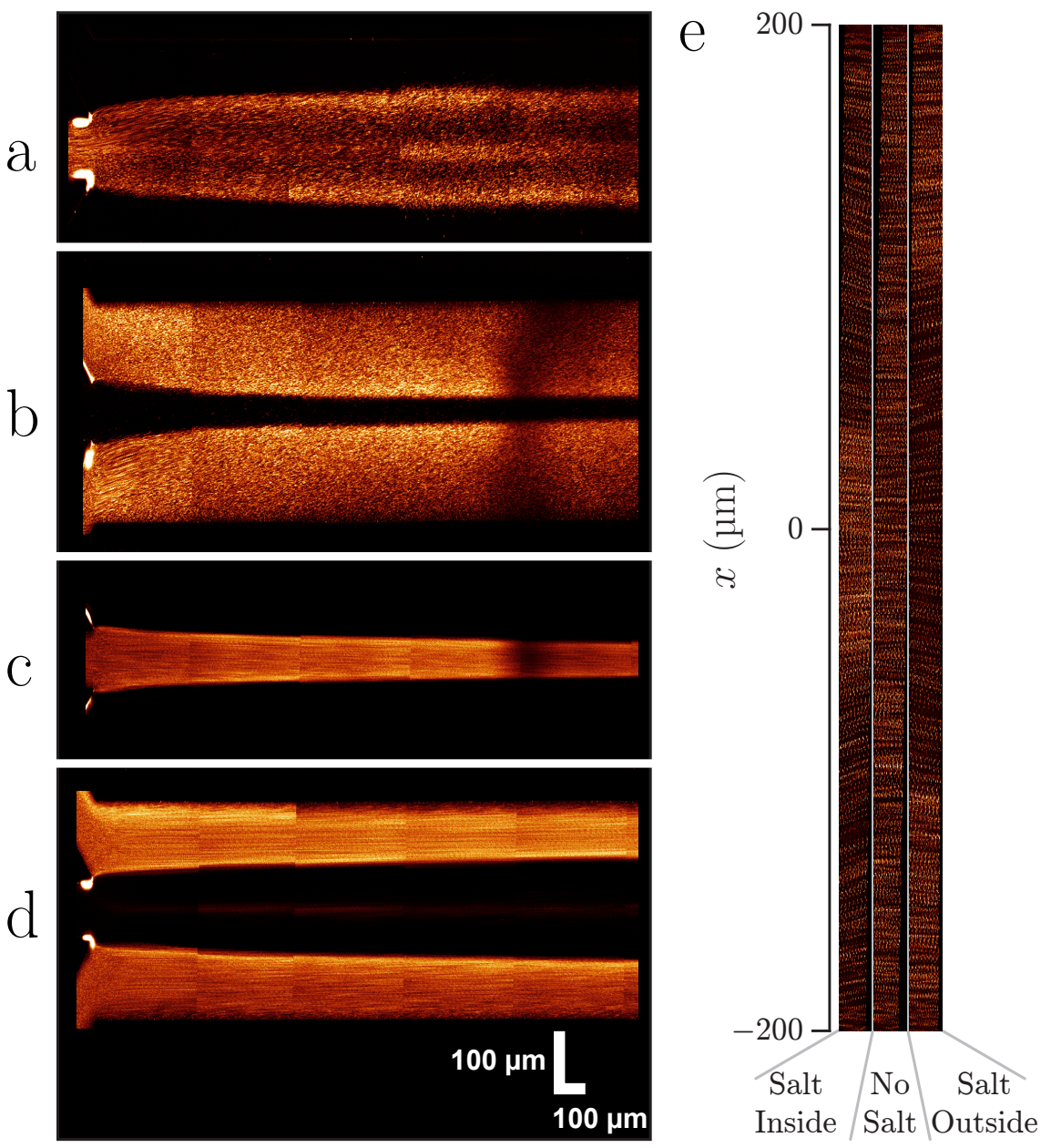

Figure 5.4: Confocal micrographs showing diffusiophoresis of silica particles in LiCl gradients. a-d) Phoretic banding of concentrated colloid streams originating in only the inner or the outer channels. a) Particles inside, salt outside. b) Particles outside, salt inside. c) Both particles and salt inside. d) Both particles and salt outside. e) Maximum intensity projections of 500 frames (10 s) captured at low concentration and high magnification to facilitate single particle tracking. A thin $y$ region located $250 \mu \mathrm{m}$ downstream from the nozzle is shown, with the projection making the trajectories discernible. Particle motion is observed to be biased towards the direction of positive salt gradient (converging in left panel, diverging in right panel). In the central panel, the particles move horizontally as there is no gradient in salt concentration. All images (a-e) have been stretched vertically by a factor of two to facilitate visualisation. The scale is identified in $(\mathrm{a}-\mathrm{d})$ by the ' $\mathrm{L}$ '-shaped scale bar and in (e), each image has dimensions $50 \times 400 \mu \mathrm{m}$. 
of single particles comprising the averaged profiles shown here.

\subsubsection{Diffusiophoresis of single particles}

An impression of the data which were taken in order to measure the diffusiophoretic velocity of single particles may be found in Figure 5.4 e. In each video frame, fewer than ten particles were visible but by stitching the videos taken across the channel and projecting hundreds of frames together, the prevailing particle trajectories become visible. Now that particles are input via all three channels, the diffusiophoretic behaviour is observable across the whole range of the solute gradient.

The directions of the trajectories show clear focussing when the high salt region is in the middle and spreading when it is at the sides. The effect is most clearly visible at approximately $\pm 100 \mu \mathrm{m}$, where the salt gradient is steepest. In the case of no salt gradient, the prevalent particle trajectory is aligned with the macroscopic flow.

By tracking around 25,000 individual particles, the diffusiophoretic velocity along $x$ was directly measured. Figure 5.5 a shows the average velocity in a series of bins along the $x$ direction, at three different sample points along $y$. Without a gradient in electrolyte concentration (in grey), the Brownian fluctuations in the $+x$ and $-x$ directions should average to 0 with equal likelihood to move in both directions. Indeed, at all three sample points along the channel, the average velocity remains close to 0 at all $x$ positions. The low amplitude noise is presumably due to the finite number of particles per bin.

With a gradient in dissolved $\mathrm{LiCl}$, a clear velocity profile emerges. The highest diffusiophoretic velocity of around $30 \mathrm{\mu m} \mathrm{s}^{-1}$ is observed at the 'earliest' sample point ( $250 \mu \mathrm{m}$ downstream) when the high salt concentration is in the channel centre. This is as expected, since the magnitude of the electrolyte gradient decreases further from the nozzle. The asymmetry in the focussing and spreading profiles is also consistent because the amount of salt introduced to the channel is not equal. In the spreading case, twice as much salt is in the channel so the gradient diminishes to a homogeneous profile more rapidly.

The experimental velocity profiles bear a striking resemblance to those predicted by Equation 5.10, shown in Figure 5.5 b, with one caveat. The measured diffusiophoretic velocities are higher than the theoretically predicted ones by a factor up to two. This discrepancy also propagates to the attempt to measure the value of the diffusiophoretic mobility across the channel (Figure $5.5 \mathrm{c}$ ), by inverting Equation 5.11 with $\nabla \ln c_{s}$ computed from Equations 5.18 and 5.19.

The same analysis of the experiments in which a $\mathrm{NaCl}$ gradient was applied is presented in Figure 5.6. The profiles are noisier but the characteristic shape of diffusiophoretic profiles is visible. The measured velocities are again higher 

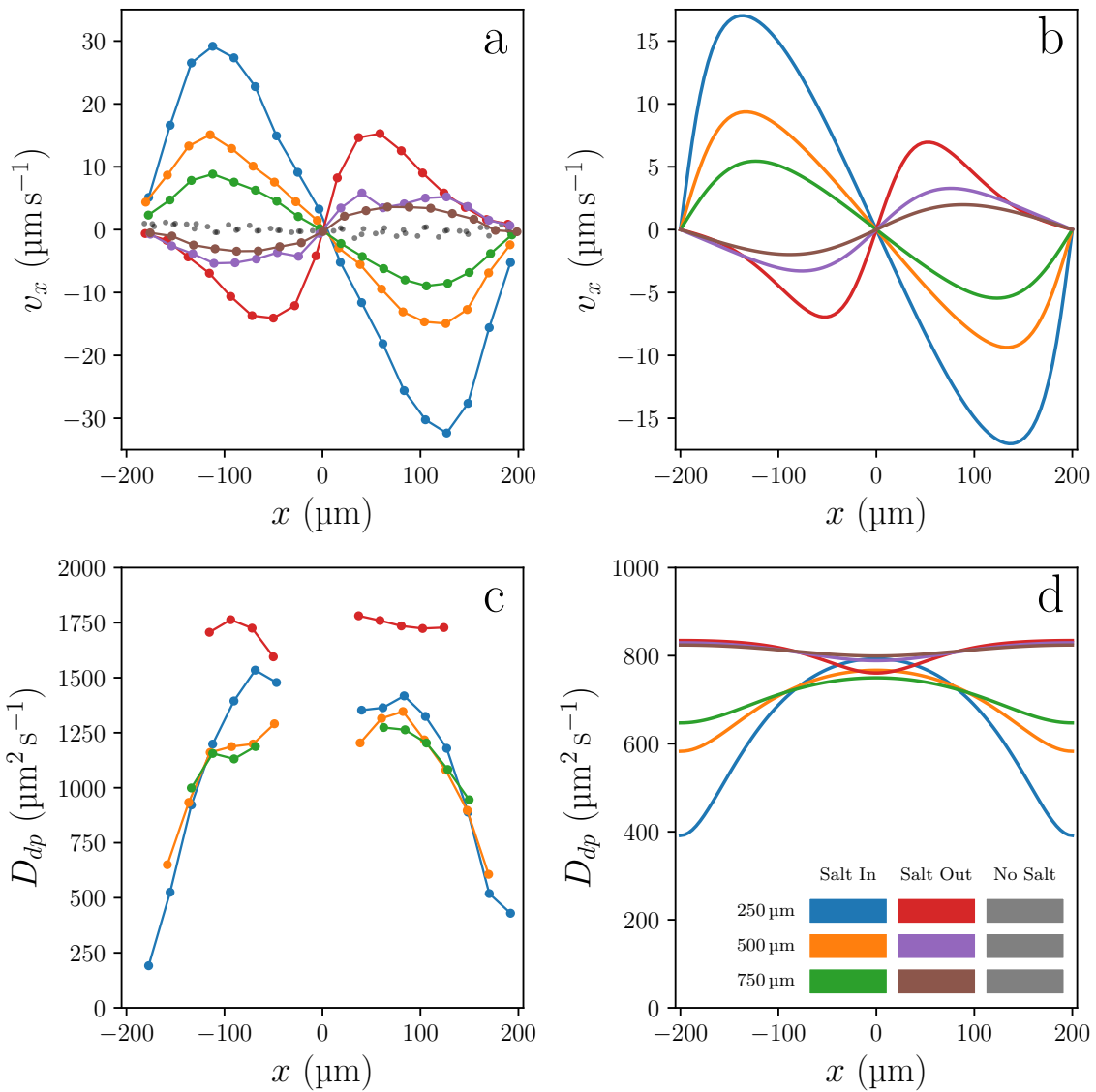

Figure 5.5: Diffusiophoresis of silica particles in $\mathrm{LiCl}$ gradient from tracking single particles (left) and theory (right). a) Average velocity transverse to the flow direction as a function of position across the channel. Straight lines connect the data to guide the eye. b) Predictions from Equation 5.10 for the same positions as in (a), using salt concentrations computed with Equations 5.18 and 5.19. c) The diffusiophoretic mobility calculated from the experimental velocities by inverting Equation 5.11 and using the same salt gradient as in (b). The points close to the centre and walls of the channel are omitted because the division by the near-zero salt gradient in these regions causes catastrophic noise amplification. d) The calculated diffusiophoretic mobility variation across the channel, which were used to compute the velocities in (b). The 2D legend in (d) applies to all four panels. The distance refers to the $y$ displacement from the nozzle and 'Salt In' indicates that the added $\mathrm{LiCl}$ was in the central channel, etc. Note that the ordinate scale is twice as large in the right panels compared to the left panels. 

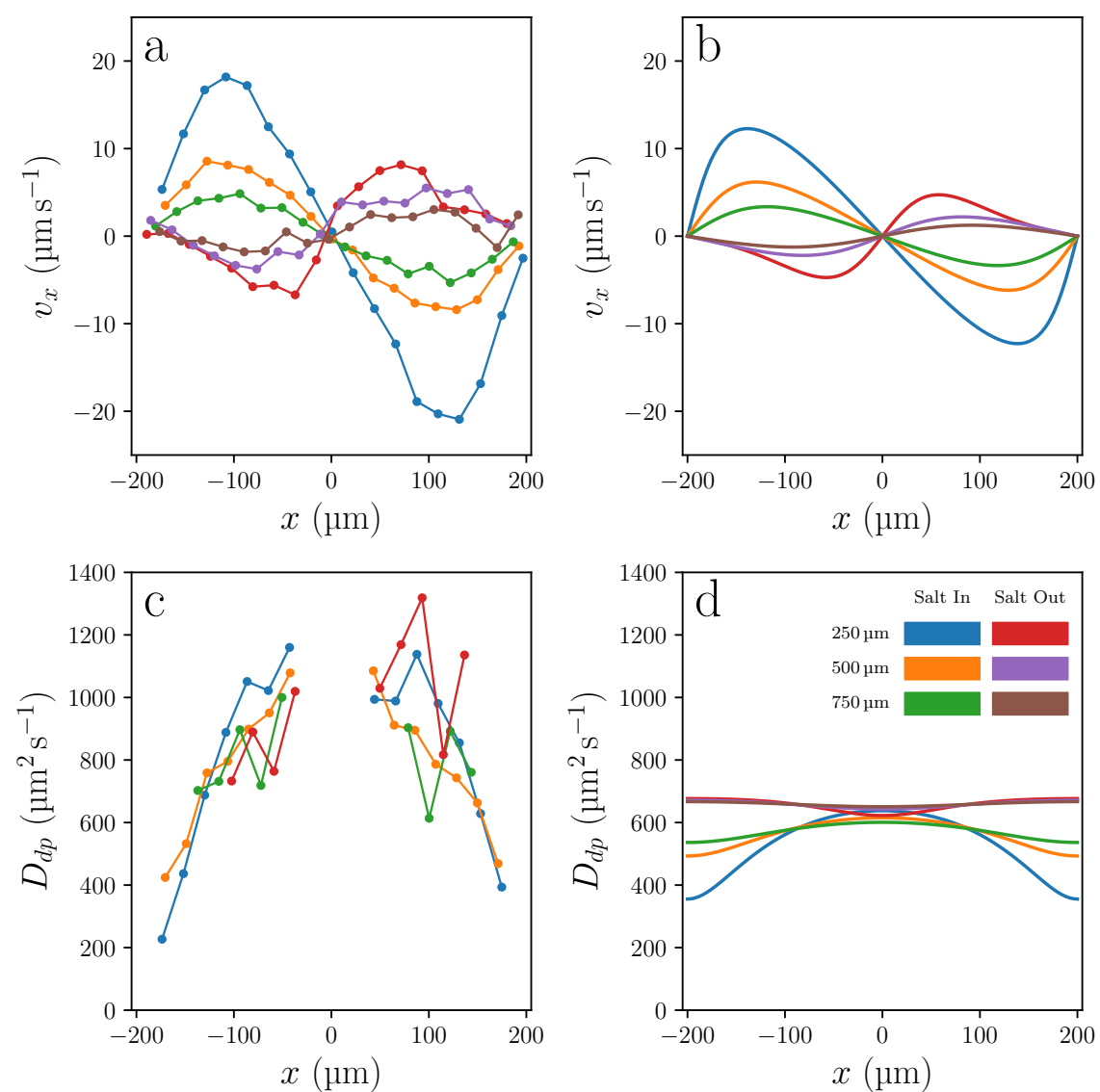

Figure 5.6: Diffusiophoresis of silica particles in $\mathrm{NaCl}$ gradient from tracking single particles (left) and theory (right). The four panels are explained in the caption of Figure 5.5. In this case, the vertical scales are the same.

than the predicted ones. With $\mathrm{NaCl}$ being a salt with less asymmetric diffusivity (lower $|\beta|$ ) than $\mathrm{LiCl}$, slower diffusiophoresis is expected in a $\mathrm{NaCl}$ gradient. This does appear to be the case when comparing the observed velocities in Figures 5.5 and 5.6 but comparing these directly is not in general valid.

The subtlety which is obscured by the apparent consistency in the aforementioned figures (and in the experimental procedure) is that of the salt concentra- 
tion profile at a given $y$ position. The different diffusivities of the salts mean that the solute profile has a different shape at (for example) $y=250 \mu \mathrm{m}$ for $\mathrm{LiCl}$ and $\mathrm{NaCl}$. The diffusiophoretic driving force, $\nabla \ln c_{s}$, has already been scaled out of Figures $5.5 \mathrm{c}$ and $5.6 \mathrm{c}$ but the 'retarding force' represented by the finite double layer and $\kappa a$ (see Figure 5.1) has not. By converting the $(x, y)$ positions in the channel to $\kappa a$ values using the calculated solute concentration profile, the diffusiophoretic mobilities for a given electrolyte should collapse onto the same curve. This is shown in Figure 5.7 a. With the mobilities now comparable, the higher values in the lithium salt gradient are confirmed.
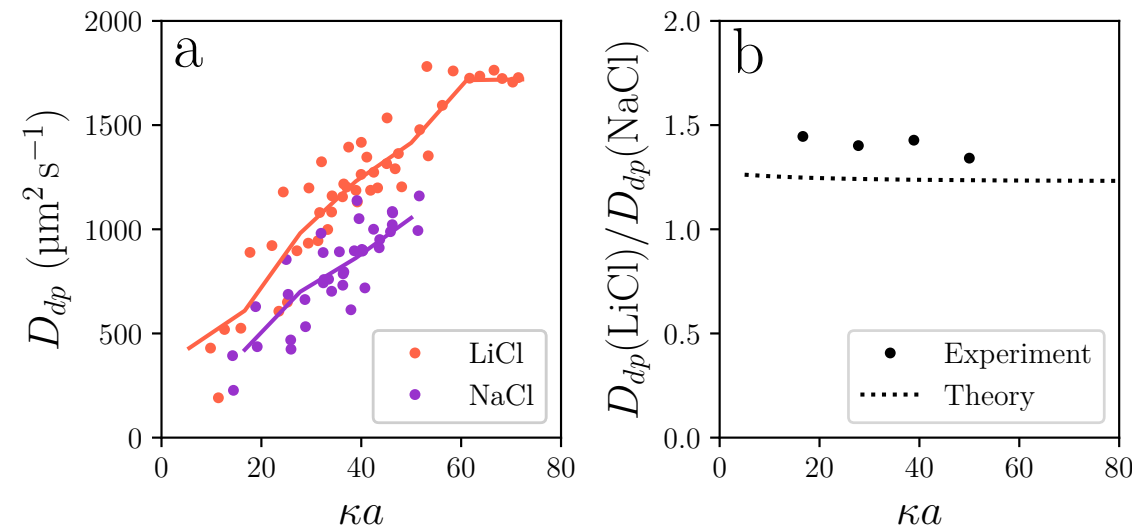

Figure 5.7: Comparison of the diffusiophoretic mobilities of the same particles in two different salt gradients. a) By computing $\kappa a(x, y)$ for the points in Figures $5.5 \mathrm{c}$ and $5.6 \mathrm{c}$, the $D_{d p}$ values between the different salts may be compared. The solid lines show the same data after averaging inside bins of width $\Delta \kappa a \sim 10$ which highlights trends and allows a ratio to be calculated for each bin. b) The ratio of the diffusiophoretic mobilities in $\mathrm{LiCl}$ gradients to those in $\mathrm{NaCl}$ gradients. The experimental ratios are approximately constant and have average 1.40. The theoretical curve is almost flat at 1.24 in this $\kappa a$ range.

The ratio of the mobilities of the same particles in the different salts is shown in Figure $5.7 \mathrm{~b}$. While the absolute values of all the measured mobilities are higher than predicted by theory, the experimental ratio (average 1.40) is close to the theoretical value (almost constant at 1.24 in the relevant $\kappa a$ range). 
Finally, diffusiophoresis was also observed in a system of TPM particles ${ }^{5}$ (Figure 5.10 in Appendix 5.A) in a LiCl gradient. Again, the measured velocities were much higher than those theoretically predicted. With the same discrepancy observed in a different system of particles, it would appear to be a systematic effect, rather than an anomalous one. With this in mind, the most likely factor in the analysis to be inaccurate is the detailed form of the salt gradient itself. This is investigated in the following section.

\subsubsection{The salt gradient}

To investigate the electrolyte concentration profile, the diffusion of a fluorescent salt was imaged in the same channel. The results are shown in Figure 5.8.

At a glance, the behaviour of the diffusing solute in Figure 5.8 a appears to match the theoretical description in Figure 5.2. However, upon inspection of the indicated transverse profiles, disparities emerge. As was noted in Section 5.3.4, the dye concentration was (mistakenly) above the self-quenching limit at the inlet and this accounts for some of the differences in the profiles. Specifically, the flattening of the fluorescence profile at its maximal values is likely due to quenching. This limits the applicability of this check on the diffusion profile to the low intensity regions, where the fluorescence intensity may still be considered proportional to the solute concentration.

In both the outward (Figure $5.8 \mathrm{~b}$ ) and inward (Figure $5.8 \mathrm{f}$ ) cases, the dye appears to spread more rapidly than the prediction of Equation 5.18 with $^{6}$ $D_{s}=639 \mathrm{\mu m}^{2} \mathrm{~s}^{-1}$. This is also visible in Figures $5.8 \mathrm{c}$ and $5.8 \mathrm{~g}$ which show the gradient of the experimental and theoretical concentration profiles. Again, the experimental profiles are likely artificially pulled towards 0 by quenching but the outer region in $\mathrm{c}$ and the inner region in $\mathrm{g}$ should be valid. In both of these, the concentration gradient is seen to have higher magnitude than the theoretical prediction.

The way in which this disparity would be expected to affect the predicted diffusiophoretic velocity is shown in Figures $5.8 \mathrm{~d}$ and $5.8 \mathrm{~h}$. Here, the ratio of the value of $\nabla \ln c$ from the dye experiment to the theoretical value is plotted. While this value diverges in the regions where the experimental dye gradient is close to 0 due to quenching, the low concentration regions may hold some insight. In the outer regions in the outward spreading experiment (Figure $5.8 \mathrm{~d}$ ), the value of this ratio fluctuates in the range $1.5-2$. This indicates is that the

\footnotetext{
${ }^{5} \varnothing 400 \mathrm{~nm} 3$-(trimethoxysilyl)propyl methacrylate spheres with $\bar{\zeta} \sim-1$ synthesised according to Reference 209.

${ }^{6}$ Calculated using the Nernst-Hartley Equation and diffusion coefficients of fluorescein and $\mathrm{Na}^{+}$in References 210 and 211 respectively.
} 
diffusiopheoretic velocity due to the measured solute gradient would be a factor of 1.5-2 greater than that due to the theoretical gradient.

The same might be occurring in Figure $5.8 \mathrm{~h}$, but the evidence is less convincing. Here, $c_{s}$ and $\nabla c_{s}$ are close to 0 in the same place, meaning that the most relevant values (at low $c_{s}$ ) are lost in the noise amplification (at low $\nabla c_{s}$ ). The values in this region are omitted from the plot.

While these experiments should clearly be repeated at lower dye concentration, the incorrect prediction of the concentration profile already seems a credible source of the disparity in the diffusiophoretic velocities.

The natural question then is why does Equation 5.18 not accurately describe the solute concentration in this well defined geometry? The place to look for an answer is at the nozzle, where theory and experiment seem most likely to disagree. There, the theory considers a perfect discontinuity at the meeting of the channels, while the real nozzle consists of micrometer sized features, without infinitely sharp points.

Figure 5.9 shows enlarged views of the nozzle during the dyed salt experiments. In Figure 5.9 a, a low concentration of dye is visible behind the $x=0$ position, which is indicative of an initial condition of the solute concentration deviating significantly from a top hat with width that of the inner channel. This different initial condition is consistent with the observed dye profile being wider than the predicted one in Figure $5.8 \mathrm{~b}$. Resolving the disparity is unfortunately not a case of replacing $w$ in Equation 5.18 with a larger effective width, because the edges of the observed profile at $x=0$ are too diffuse to be approximated by a wider top hat function. Numerical solution of Equation 5.16 with a more complex initial condition might reconcile the theoretical profile with the experiments, although this seems a rather unsatisfying hack. Besides, a procedure to convert the initial dye profile to that of a salt of different diffusivity etc. is not obvious without a clear understanding of the effect causing this broadening.

The lack of a corresponding dark region behind the nozzle in Figure $5.9 \mathrm{~b}$ rules out a purely hydrodynamic effect resulting from the nozzle geometry and flow rates. The most likely mechanism, therefore, would seem to be the very one being investigated, except in a different frame of reference.

Diffusiophoresis occurs as a result of gradient induced flow along the surface of a colloidal particle as discussed in Section 5.2.1. The particle sees itself as stationary with the liquid flowing by at the slip velocity. In the laboratory frame of reference, the particle is observed to move towards higher solute concentration. If the surface in question were fixed in the laboratory frame (for example: the wall of a microfluidic device), then a flow would be observed in the direction of decreasing solute concentration. This phenomenon is called 

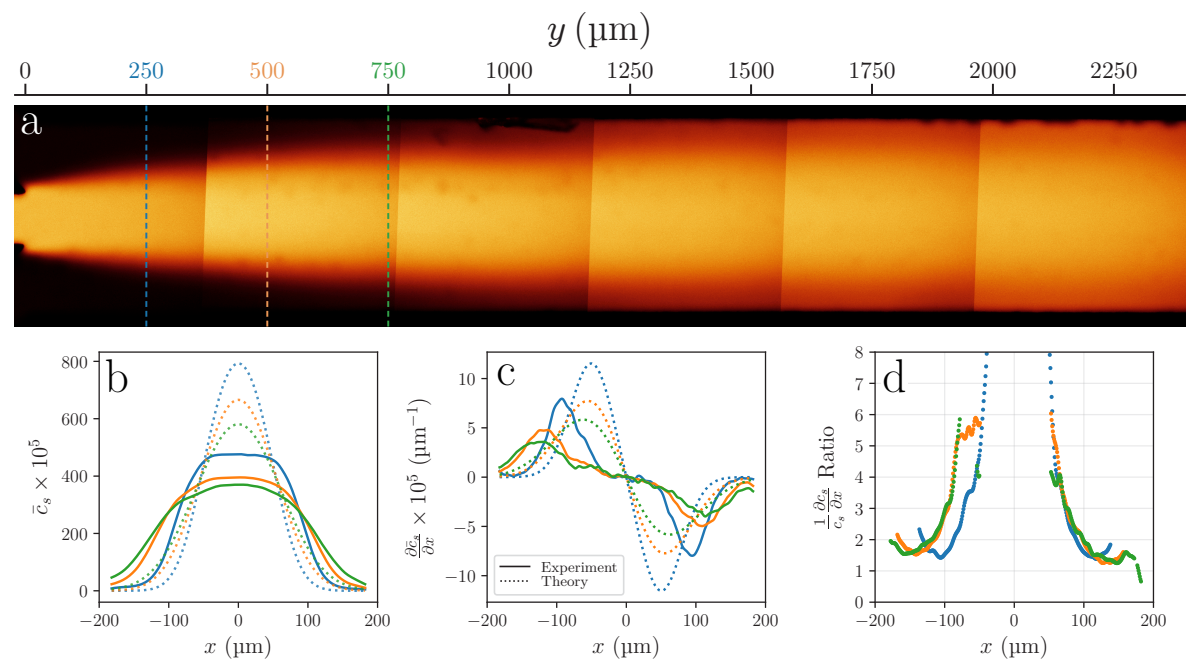

$y(\mu \mathrm{m})$
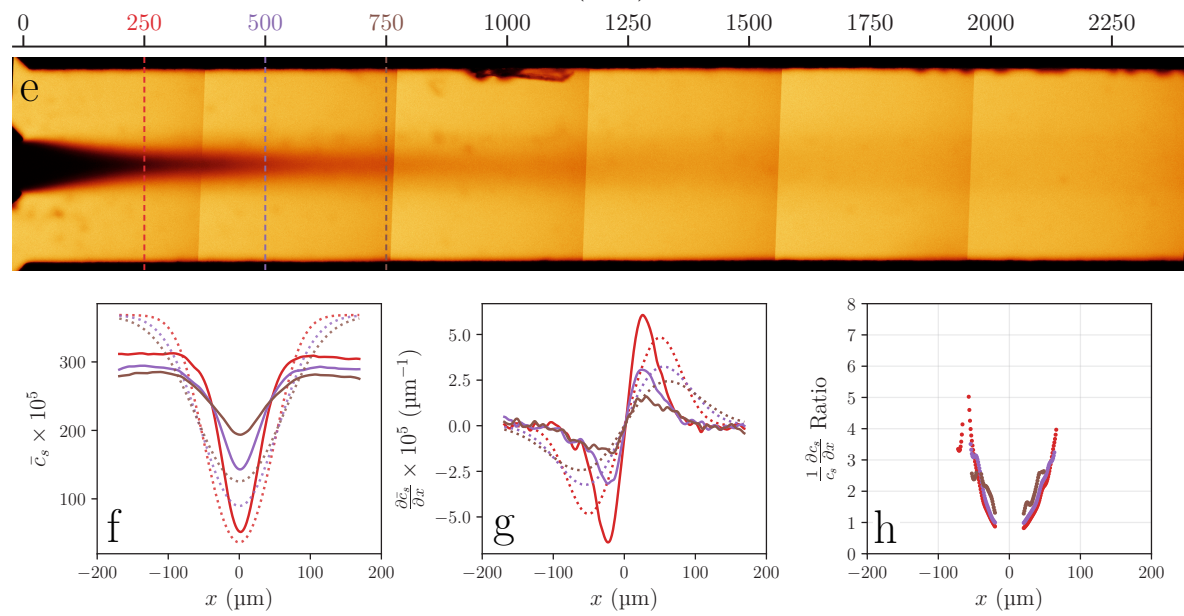

Figure 5.8: Examining salt profiles with a fluorescent dye (and no particles). a-d) Dye enters via the inner channels and diffuses outwards. e- $\mathrm{h}$ ) Dye enters via the outer channels and diffuses inwards. a,e) Stitched confocal images showing $\sim 2 \mathrm{~mm}$ of the channel length. b,f) Line profiles at distances 250, 500 and $700 \mathrm{\mu m}$ from the nozzle as indicated by the dashed lines across the images. Solid lines show experimental profiles and dotted lines are concentrations calculated using Equation 5.18. $\bar{c}_{s}$ denotes the concentration (fluorescence intensity) normalised by the total intensity in the line. $\mathrm{c}, \mathrm{g}$ ) The gradient in salt concentration from line profiles (solid) or calculated with Equation 5.19 (dotted). d, h) The ratio of $\nabla \ln c_{s}$ observed for the dye to the theoretical value. 

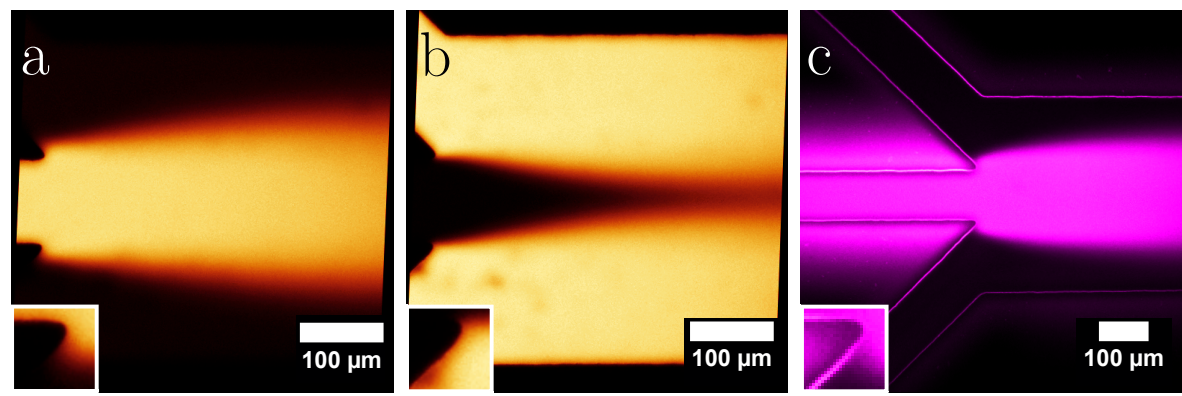

Figure 5.9: Enlarged views at the nozzle in dye diffusion experiments. a) Sodium fluorescein dye enters via central channel. b) Dye enters via outer channels. c) Fluorescence image of a preliminary experiment with rhodamine B instead of sodium fluorescein, in which the dye penetrates the PDMS walls [214]. A different lookup table was is used to distinguish this from the main fluorescein experiments. The insets show enlarged views of the lower confluence point and all have side length $40 \mu \mathrm{m}$.

diffusio-osmosis ${ }^{7}$ [212] and it is to diffusiophoresis what electroosmosis is to electrophoresis [174, 178].

Given that the radius of curvature of the PDMS wall shown in the insets of Figure 5.9 is orders of magnitude greater than $\kappa^{-1}$, the slip velocity at this wall should be well described by the sum of Equations 5.5 and 5.6. The zeta potential of PDMS is similar to that of the colloidal silica [213] and the almost discontinuous solute gradient at the very point of confluence might drive a very large slip velocity indeed. Thus it is concluded that the solute gradient was disrupted by a 'slinging' flow around the corner of the nozzle, induced by the solute gradient. Such are the perils of surface science in a system which is necessarily bounded by surfaces.

The reasons for the disparity between the experimental and theoretical solute profiles are certainly interesting but the most important conclusion from this section is that there $i s$ a disparity. Thus the electrolyte gradient in the diffusiophoresis was not exactly known, ruling out the possibility of reconciling the magnitude of the diffusiophoretic velocity in experiment and theory.

\footnotetext{
${ }^{7}$ Or 'capillary osmosis' [169].
} 


\subsection{Conclusions}

It has been demonstrated that a combination of a microfluidic $\psi$ channel and single particle tracking may be used to directly measure diffusiophoretic velocities. The results were compared with theory and the fidelity of the measurements was such that subtle details of the predicted velocity profiles were identifiable in the experimental ones. The magnitudes of the experimental diffusiophoretic velocities were found to be consistently higher than those predicted by theory. This was ascribed to differences between the theoretical and experimental solute gradients leading to an underestimation of the diffusiophoretic driving force. With minor modifications to bring the solute gradient under control, it is likely that this method will prove powerful in measuring the detailed responses of colloidal particles to solute gradients.

\subsection{Outlook}

The use of a glass device such as that used in Reference 196 with sharper features in the nozzle would likely allow for a much better approximation of a step in solute concentration at the nozzle. A convenient internal control might be to combine the two main experiments of this chapter. Namely, by using a fluorescent electrolyte gradient to drive diffusiophoresis, both the particle motion and the solute gradient might be measured simultaneously. This would require a dye of higher diffusivity and higher self-quenching concentration than the one used in this chapter.

Once the issue of the solute gradient has been ironed out, it is envisioned that the method described in this chapter might become a standard laboratory technique to measure diffusiophoretic mobilities, in analogy to capillary electrophoresis. What has not yet been fully demonstrated here is the considerable versatility of the method. For example, diffusiophoretic mobilities could be measured from a very small quantity of non-fluorescent particles, utilising the reflection signal from single particles in very dilute dispersion.

\section{Acknowledgements}

This work was initiated by Pepijn Moerman and carried out in collaboration with the same. Relinde van Dijk-Moes is acknowledged for synthesising the silica particles and Ramakrishna Kotni for the T P M particles. Alessia Broccoli and Mathieu Odijk of the University of Twente are thanked for fabricating the master mould from which the $\psi$ channels were cast. An anonymous member of 
the Van 't Hoff Laboratory is thanked for a clandestine donation of a few milligrams of sodium fluorescein. Finally, financial support in the form of partial sponsorship of the course 'Introduction to Microfluidics' course at Brandeis University is acknowledged (NSFDMR1420382). 


\section{A Diffusiophoresis of TPM particles}
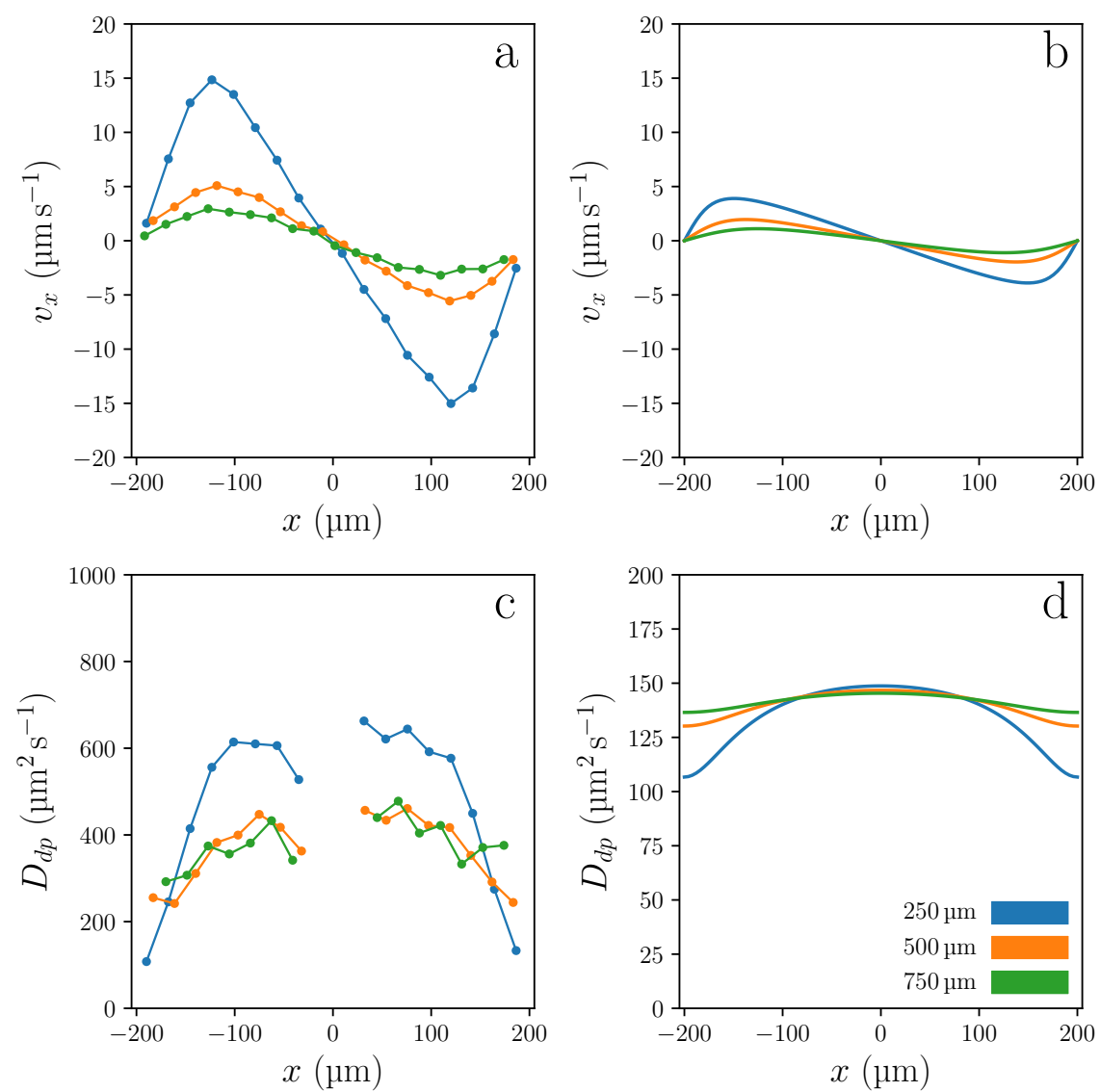

Figure 5.10: Diffusiophoresis of T P M particles in LiCl gradient from tracking single particles (left) and theory (right). The four panels are explained in the caption of Figure 5.5. 


\section{Bibliography}

[1] R. Piazza. Soft Matter: The stuff that dreams are made of. Springer, 2011. DOI: $10.1007 / 978-94-007-0585-2$. (see p. 2)

[2] G.V. Franks, C. Tallon, A.R. Studart, M.L. Sesso and S. Leo. 'Colloidal processing: enabling complex shaped ceramics with unique multiscale structures'. Journal of the American Ceramic Society 100: 458-490, 2017. DOI: $10.1111 /$ jace.14705. (see p. 3)

[3] G. Bickley Remmey. Firing Ceramics. World Scientific, 1994. DoI: 10. 1142/2279. (see p. 3)

[4] B. Vincent. 'Introduction to Colloidal Dispersions'. In: Colloid Science. Blackwell Publishing Ltd., 2005. 1-13. DOI: 10.1002/9781444305395. ch1. (see p. 4)

[5] R.J. Hunter. Foundations of Colloid Science. 2nd ed. Oxford University Press, 2001. Chap. 1: Nature of colloidal dispersions, 304-372. (see p. 4)

[6] H.C. Sorby. 'On the organic origin of the so-called 'Crystalloids' of the chalk'. Annals and Magazine of Natural History 8: 193-200, 1861. DOI: 10.1080/00222936108697404. (see p. 5)

[7] F.M. Ross. 'Opportunities and challenges in liquid cell electron microscopy'. Science 350: 9886, 2015. DOI: 10.1126/science.aaa9886. (see p. 6)

[8] L. Schermelleh, A. Ferrand, T. Huser, C. Eggeling, M. Sauer, O. Biehlmaier and G.P.C. Drummen. 'Super-resolution microscopy demystified'. Nature Cell Biology 21: 72-84, 2019. DOI: 10.1038/s41556-018-02518. (see p. 6)

[9] J.L. Anderson. 'Colloid Transport by Interfacial Forces'. Annual Review of Fluid Mechanics 21: 61-99, 1989. DOI: 10.1146/annurev.fl.21.010189. 000425. (see pp. 6, 7, 116)

[10] T. Aste and D. WeAiRE. The pursuit of perfect packing. Bristol, PA: Institute of Physics Publishing, 2000. (see p. 10)

[11] R. Piazza. 'Settled and unsettled issues in particle settling'. Reports on Progress in Physics 77: 056602, 2014. DOI: 10.1088/0034-4885/77/5/ 056602. (see p. 10)

[12] B.J. Alder and T.E. Wainwright. 'Phase Transition for a Hard Sphere System'. The Journal of Chemical Physics 27: 1208-1209, 1957. DOI: 10. 1063/1.1743957. (see p. 11) 
Bibliography

[13] P.N. Pusey and W. van Megen. 'Phase behaviour of concentrated suspensions of nearly hard colloidal spheres'. Nature 320: 340-342, 1986. DOI: 10.1038/320340a0. (see p. 11)

[14] P.N. Pusey. 'The effect of polydispersity on the crystallization of hard spherical colloids'. Journal de Physique 48: 709-712, 1987. DOI: 10.1051/ jphys:01987004805070900. (see p. 11)

[15] E. Zaccarelli, C. Valeriani, E. Sanz, W.C.K. Poon, M.E. Cates and P.N. Pusey. 'Crystallization of Hard-Sphere Glasses'. Physical Review Letters 103: 2009. DOI: 10.1103/physrevlett.103.135704. (see p. 11)

[16] P. Bartlett and P.B. Warren. 'Reentrant Melting in Polydispersed Hard Spheres'. Physical Review Letters 82: 1979-1982, 1999. DOI: $10.1103 /$ physrevlett.82.1979. (see p. 12)

[17] D.A. Kofke and P.G. Bolhuis. 'Freezing of polydisperse hard spheres'. Physical Review E 59: 618-622, 1999. DOI: 10.1103/physreve.59.618. (see pp. 12, 50)

[18] P. Chaudhuri, S. Karmakar, C. Dasgupta, H.R. Krishnamurthy and A.K. Sood. 'Equilibrium Glassy Phase in a Polydisperse Hard-Sphere System'. Physical Review Letters 95: 2005. DOI: 10.1103/physrevlett. 95. 248301. (see p. 12)

[19] P. Sollich and N.B. Wilding. 'Crystalline Phases of Polydisperse Spheres'. Physical Review Letters 104: 2010. DOI: 10.1103/physrevlett.104.118302. (see p. 12)

[20] B. Cabane, J. Li, F. Artzner, R. Botet, C. Labbez, G. Bareigts, M. Sztucki and L. Goenring. 'Hiding in Plain View: Colloidal Self-Assembly from Polydisperse Populations'. Physical Review Letters 116: 2016. DOI: 10.1103/physrevlett.116.208001. (see p. 12)

[21] B.A. Lindquist, R.B. Jadrich and T.M. Truskett. 'From close-packed to topologically close-packed: Formation of Laves phases in moderately polydisperse hard-sphere mixtures'. The Journal of Chemical Physics 148: 191101, 2018. DOI: 10.1063/1.5028279. (see p. 12)

[22] P.K. Bommineni, N.R. Varela-Rosales, M. Klement and M. Engel. 'Complex Crystals from Size-Disperse Spheres'. Physical Review Letters 122: 2019. DOI: 10.1103/physrevlett.122.128005. (see p. 12)

[23] Y. Xia, B. Gates, Y. Yin and Y. Lu. 'Monodispersed Colloidal Spheres: Old Materials with New Applications'. Advanced Materials 12: 693-713, 2000. DOI: $10.1002 /$ (sici) 1521-4095(200005)12:10<693: :aid-adma693> 3.0.co;2-j. (see p. 12)

[24] W. StöBer, A. Fink and E. Bohn. 'Controlled growth of monodisperse silica spheres in the micron size range'. Journal of Colloid and Interface Science 26: 62-69, 1968. DOI: 10.1016/0021-9797(68)90272-5. (see p. 12) 
[25] A. van Blaaderen, J. van Geest and A. Vrij. 'Monodisperse colloidal silica spheres from tetraalkoxysilanes: particle formation and growth mechanism'. Journal of Colloid and Interface Science 154: 481-501, 1992. DOI: 10.1016/0021-9797 (92)90163-G. (see pp. 12, 17, 18, 33, 82)

[26] H. Giesche. 'Synthesis of monodispersed silica powders II. Controlled growth reaction and continuous production process'. Journal of the European Ceramic Society 14: 205-214, 1994. DOI: 10.1016/0955-2219(94) 90088-4. (see pp. 12, 23, 126)

[27] A. van BlaAderen and A. VRiJ. 'Synthesis and characterization of colloidal dispersions of fluorescent, monodisperse silica spheres'. Langmuir 8: 29212931, 1992. DOI: 10.1021/1a00048a013. (see pp. 12, 22, 45, 126)

[28] T. Yokoi, Y. Sakamoto, O. Terasaki, Y. Kubota, T. Okubo and T. Tatsumi. 'Periodic Arrangement of Silica Nanospheres Assisted by Amino Acids'. Journal of the American Chemical Society 128: 13664-13665, 2006. DOI: $10.1021 /$ ja065071y. (see p. 12)

[29] K.D. Hartlen, A.P.T. Athanasopoulos and V. Kitaev. 'Facile Preparation of Highly Monodisperse Small Silica Spheres $(15$ to $>200 \mathrm{~nm})$ Suitable for Colloidal Templating and Formation of Ordered Arrays'. Langmuir 24: 1714-1720, 2008. DOI: 10.1021/la7025285. (see pp. 12, 20, 29, 31, 34, 45)

[30] H.S. Lee, T.S. Shim, H. Hwang, S.-M. Yang and S.-H. Kim. 'Colloidal Photonic Crystals toward Structural Color Palettes for Security Materials'. Chemistry of Materials 25: 2684-2690, 2013. DOI: 10.1021/cm4012603. (see p. 12)

[31] C.H. Lim, H. Kang and S.-H. Kim. 'Colloidal Assembly in Leidenfrost Drops for Noniridescent Structural Color Pigments'. Langmuir 30: 8350-8356, 2014. DOI: 10.1021/la502157p. (see p. 12)

[32] A. Zhokhov, V. Masalov, N. Sukhinina, D. Matveev, P. Dolganov, V. Dolganov and G. Emelchenko. 'Photonic crystal microspheres'. Optical Materials 49: 208-212, 2015. DOI: 10.1016/j.optmat.2015.09.019. (see p. 12)

[33] G.H. Lee, T.M. Choi, B. Kim, S.H. Han, J.M. Lee and S.-H. Kim. 'Chameleon-Inspired Mechanochromic Photonic Films Composed of NonClose-Packed Colloidal Arrays'. ACS Nano 11: 11350-11357, 2017. DOI: 10.1021/acsnano.7b05885. (see p. 12)

[34] H. Lee, T.Y. Jeon, S.Y. LeE, S.Y. LeE and S.-H. Kim. 'Designing Multicolor Micropatterns of Inverse Opals with Photonic Bandgap and Surface Plasmon Resonance'. Advanced Functional Materials 28: 1706664, 2018. DOI: 10.1002/adfm.201706664. (see p. 12)

[35] J.-W. Kim, J.-S. LeE and S.-H. Kim. 'Biodegradable Inverse Opals with Controlled Discoloration'. Advanced Materials Interfaces 5: 1701658, 2018. DOI: 10.1002/admi.201701658. (see p. 12) 
Bibliography

[36] G.H. Lee, J.B. Kim, T.M. Choi, J.M. LeE and S.-H. Kim. 'Structural Coloration with Nonclose-Packed Array of Bidisperse Colloidal Particles'. Small 15: 1804548, 2019. DOI: 10.1002/smll.201804548. (see p. 12)

[37] G.H. Lee, S.H. Han, J.B. Kim, J.H. Kim, J.M. LeE and S.-H. Kim. 'Colloidal Photonic Inks for Mechanochromic Films and Patterns with Structural Colors of High Saturation'. Chemistry of Materials 31: 8154-8162, 2019. DOI: 10.1021/acs. chemmater.9b02938. (see p. 12)

[38] S. Shahabi, L. Treccani and K. Rezwan. 'Amino acid-catalyzed seed regrowth synthesis of photostable high fluorescent silica nanoparticles with tunable sizes for intracellular studies'. Journal of Nanoparticle Research 17: 2015. DOI: $10.1007 /$ s11051-015-3072-8. (see pp. 12, 13, 19)

[39] T. Kao, F. Kohle, K. Ma, T. Aubert, A. Andrievsky and U. Wiesner. 'Fluorescent Silica Nanoparticles with Well-Separated Intensity Distributions from Batch Reactions'. Nano Letters 18: 1305-1310, 2018. DoI: 10.1021/ acs.nanolett.7b04978. (see p. 13)

[40] N.A.M. Verhaegh and A. van BlaAderen. 'Dispersions of RhodamineLabeled Silica Spheres: Synthesis, Characterization, and Fluorescence Confocal Scanning Laser Microscopy'. Langmuir 10: 1427-1438, 1994. DOI: 10.1021/1a00017a019. (see pp. 13, 126)

[41] T.M. Davis, M.A. Snyder, J.E. Krohn and M. Tsapatsis. 'Nanoparticles in Lysine-Silica Sols'. Chemistry of Materials 18: 5814-5816, 2006. DOI: 10.1021/cm061982v. (see p. 13)

[42] M.A. Snyder, J.A. Lee, T.M. Davis, L.E. Scriven and M. Tsapatsis. 'Silica Nanoparticle Crystals and Ordered Coatings Using Lys-Sil and a Novel Coating Device'. Langmuir 23: 9924-9928, 2007. DOI: 10.1021/1a701063v. (see p. 13)

[43] T. Yokoi, J. Wakabayashi, Y. Otsuka, W. Fan, M. Inama, R. Watanabe, K. Aramaki, A. Shimojima, T. Tatsumi and T. Okubo. 'Mechanism of Formation of Uniform-Sized Silica Nanospheres Catalyzed by Basic Amino Acids'. Chemistry of Materials 21: 3719-3729, 2009. DOI: $10.1021 /$ cm900993b. (see pp. 13, 14)

[44] J. Wang, A. Sugawara, A. Shimojima and T. Okubo. 'Preparation of Anisotropic Silica Nanoparticles via Controlled Assembly of Presynthesized Spherical Seeds'. Langmuir 26: 18491-18498, 2010. DOI: 10.1021/ la103564p. (see pp. 13, 33)

[45] R. Watanabe, T. Yokoi, E. Kobayashi, Y. Otsuka, A. Shimojima, T. OkuBo and T. TAtsumi. 'Extension of size of monodisperse silica nanospheres and their well-ordered assembly'. Journal of Colloid and Interface Science 360: 1-7, 2011. DOI: $10.1016 / \mathrm{j}$.jcis.2010.09.001. (see p. 13) 
[46] S. Foullloux, A. Désert, O. Taché, O. Spalla, J. Daillant and A. THILL. 'SAXS exploration of the synthesis of ultra monodisperse silica nanoparticles and quantitative nucleation growth modeling'. Journal of Colloid and Interface Science 346: 79-86, 2010. DOI: 10.1016/j.jcis.2010.02.052. (see p. 14)

[47] S. Fouilloux, O. Taché, O. Spalla and A. Thill. 'Nucleation of Silica Nanoparticles Measured in Situ during Controlled Supersaturation Increase. Restructuring toward a Monodisperse Nonspherical Shape'. Langmuir 27: 12304-12311, 2011. DOI: 10.1021/la2013842. (see p. 14)

[48] S. Fouilloux, J. Daillant and A. Thill. 'Single step synthesis of 5-30nm monodisperse silica nanoparticles: Important experimental parameters and modeling'. Colloids and Surfaces A: Physicochemical and Engineering Aspects 393: 122-127, 2012. DOI: 10.1016/j.colsurfa.2011.11.009. (see p. 14)

[49] C.C.M.C. CArcouËt et al. 'Nucleation and Growth of Monodisperse Silica Nanoparticles'. Nano Letters 14: 1433-1438, 2014. DOI: 10.1021/nl404550d. (see p. 14)

[50] G.H. Bogush and C.F. Zukoski. 'Studies of the kinetics of the precipitation of uniform silica particles through the hydrolysis and condensation of silicon alkoxides'. Journal of Colloid and Interface Science 142: 1-18, 1991. DOI: 10.1016/0021-9797(91)90029-8. (see p. 14)

[51] R. Bertholdo, F.V. dos Reis, S.H. Pulcinelli and C.V. Santilli. 'SAXS study of monodispersed silica nanospheres obtained by an amino acid route'. Journal of Non-Crystalline Solids 356: 2622-2625, 2010. DOI: $10.1016 / \mathrm{j}$. jnoncrysol.2010.05.011. (see p. 14)

[52] E.D.E.R. Hyde, A. Seyfaee, F. Neville and R. Moreno-Atanasio. 'Colloidal Silica Particle Synthesis and Future Industrial Manufacturing Pathways: A Review'. Industrial \& Engineering Chemistry Research 55: 8891-8913, 2016. DOI: 10.1021/acs.iecr.6b01839. (see pp. 16, 31,33)

[53] J. Bailey and M. Mecartney. 'Formation of colloidal silica particles from alkoxides'. Colloids and Surfaces 63: 151-161, 1992. DOI: 10.1016/01666622(92)80081-c. (see p. 17)

[54] A. van BlaAderen and A. Kentgens. 'Particle morphology and chemical microstructure of colloidal silica spheres made from alkoxysilanes'. Journal of Non-Crystalline Solids 149: 161-178, 1992. DOI: 10.1016/0022-3093(92) 90064-q. (see pp. 17, 33)

[55] H. Giesche. 'Synthesis of monodispersed silica powders I. Particle properties and reaction kinetics'. Journal of the European Ceramic Society 14: 189-204, 1994. DOI: 10.1016/0955-2219(94)90087-6. (see p. 17) 
[56] T. Matsoukas and E. Gulari. 'Monomer-addition growth with a slow initiation step: A growth model for silica particles from alkoxides'. Journal of Colloid and Interface Science 132: 13-21, 1989. DOI: 10.1016/00219797 (89)90210-5. (see pp. 17, 18)

[57] T. Matsoukas and E. Gulari. 'Self-sharpening distributions revisited-polydispersity in growth by monomer addition'. Journal of Colloid and Interface Science 145: 557-562, 1991. DOI: 10.1016/0021-9797(91)90385-1. (see pp. 17, 18)

[58] K.D. Keefer. 'Growth and Structure of Fractally Rough Silica Colloids'. MRS Proceedings 73: 1986. DOI: 10.1557/proc-73-295. (see p. 18)

[59] J.W. Vanderhoff, J.F. Vitkuske, E.B. Bradford and T. Alfrey. 'Some factors involved in the preparation of uniform particle size latexes'. Journal of Polymer Science 20: 225-234, 1956. DOI: 10.1002/pol.1956.120209501. (see p. 18)

[60] J.H. Zhang, P. Zhan, Z.L. Wang, W.Y. Zhang and N.B. Ming. 'Preparation of monodisperse silica particles with controllable size and shape'. Journal of Materials Research 18: 649-653, 2003. DOI: 10.1557/jmr.2003.0085. (see p. 23)

[61] B. Liu, T.H. Besseling, M. Hermes, A.F. Demirörs, A. Imhof and A. VAN BLAADEREN. 'Switching plastic crystals of colloidal rods with electric fields'. Nature Communications 5: 2014. DOI: 10.1038/ncomms4092. (see p. 24)

[62] P. Jiang, J.F. Bertone, K.S. Hwang and V.L. Colvin. 'Single-Crystal Colloidal Multilayers of Controlled Thickness'. Chemistry of Materials 11: 2132-2140, 1999. DOI: 10.1021/cm990080+. (see p. 25)

[63] Glycerine Producers' Association. Physical properties of glycerine and its solutions. New York, 1963. (see pp. 25, 83)

[64] D. Allan et al. soft-matter/trackpy: Trackpy v0.4.2. 2019. DOI: 10.5281/ ZENODO.3492186 (see pp. 25, 128)

[65] J.C. Crocker and D.G. Grier. 'Methods of Digital Video Microscopy for Colloidal Studies'. Journal of Colloid and Interface Science 179: 298-310, 1996. DOI: $10.1006 /$ jcis.1996.0217. (see pp. 25, 128)

[66] A. Stukowski. 'Visualization and analysis of atomistic simulation data with OVITO-the Open Visualization Tool'. Modelling and Simulation in Materials Science and Engineering 18: 015012, 2009. DOI: 10.1088/09650393/18/1/015012. (see p. 25)

[67] D. FAKen and H. Jónsson. 'Systematic analysis of local atomic structure combined with 3D computer graphics'. Computational Materials Science 2: 279-286, 1994. DOI: 10.1016/0927-0256(94)90109-0. (see p. 25) 
[68] Ü. Akbey, B. Altin, A. Linden, S. Özçelik, M. Gradzielski and H. Oschkinat. 'Dynamic nuclear polarization of spherical nanoparticles'. Physical Chemistry Chemical Physics 15: 20706, 2013. DOI: 10.1039/c3cp53095g. (see pp. 31, 45)

[69] J.L. Russell, N.-L.L. Tran and T.E. Mallouk. 'Adaptive Shape Ripening and Interparticle Bridging of l-Arginine-Stabilized Silica Nanoparticles during Evaporative Colloidal Crystal Assembly'. ACS Applied Materials \& Interfaces 11: 4568-4577, 2019. DOI: 10.1021/acsami.8b17907. (see pp. 31, 34)

[70] P.F. Marina, C. Cheng, R. Sedev, A. Stocco, B.P. Binks and D. Wang. 'Van der Waals Emulsions: Emulsions Stabilized by Surface-Inactive, Hydrophilic Particles via van der Waals Attraction'. Angewandte Chemie International Edition 57: 9510-9514, 2018. DOI: 10.1002/anie.201805410. (see p. 31)

[71] E.J.W. Verwey. 'Theory of the Stability of Lyophobic Colloids'. The Journal of Physical and Colloid Chemistry 51: 631-636, 1947. DOI: 10 . 1021/j150453a001. (see p. 33)

[72] T. Cosgrove, ed. Colloid Science. Blackwell Publishing Ltd., Aug. 2005. DOI: 10.1002/9781444305395. (see pp. 33, 171)

[73] M. Yang, G. Chen, Y. Zhao, G. Silber, Y. Wang, S. Xing, Y. Han and H. CHEN. 'Mechanistic investigation into the spontaneous linear assembly of gold nanospheres'. Physical Chemistry Chemical Physics 12: 11850, 2010. DOI: $10.1039 /$ c0cp00127a. (see p. 33)

[74] J. Liao, Y. Zhang, W. Yu, L. Xu, C. Ge, J. Liu and N. Gu. 'Linear aggregation of gold nanoparticles in ethanol'. Colloids and Surfaces A: Physicochemical and Engineering Aspects 223: 177-183, 2003. DOI: 10.1016/s09277757(03)00156-0. (see p. 33)

[75] X. Han, J. Goebl, Z. Lu and Y. Yin. 'Role of Salt in the Spontaneous Assembly of Charged Gold Nanoparticles in Ethanol'. Langmuir 27: 52825289, 2011. DOI: 10.1021/la200459t. (see p. 33)

[76] G. Trefalt and M. Borkovec. Overview of DLVO Theory. 2014. urL: www. colloid.ch/dlvo accessed 24/03/2020 (see p. 33)

[77] A. Dong, J. Chen, P.M. Vora, J.M. Kikkawa and C.B. Murray. 'Binary nanocrystal superlattice membranes self-assembled at the liquid-air interface'. Nature 466: 474-477, 2010. DOI: 10.1038/nature09188. (see p. 42)

[78] U. Landman and W.D. LuedtKe. 'Small is different: energetic, structural, thermal, and mechanical properties of passivated nanocluster assemblies'. Faraday Discussions 125: 1, 2004. DOI: 10.1039/b312640b. (see p. 42) 
Bibliography

[79] X. Cao, E. Panizon, A. Vanossi, N. Manini and C. Bechinger. 'Orientational and directional locking of colloidal clusters driven across periodic surfaces'. Nature Physics 15: 776-780, 2019. DOI: 10.1038/s41567-0190515-7. (see p. 43)

[80] H. Maeda, N. Ishida, H. Kawauchi and K. Tuzimura. 'Reaction of Fluorescein-Isothiocyanate with Proteins and Amino Acids: I. Covalent and Non-Covalent Binding of Fluorescein-Isothiocyanate and Fluorescein to Proteins'. The Journal of Biochemistry 65: 777-783, 1969. DOI: $10.1093 /$ oxfordjournals. jbchem.a129077. (see p. 45)

[81] S. Shahabi, L. Treccani and K. Rezwan. 'A comparative study of three different synthesis routes for hydrophilic fluorophore-doped silica nanoparticles'. Journal of Nanoparticle Research 18: 2016. DOI: 10.1007/s11051-0163334-0. (see p. 45)

[82] G.T. Vladisavluević. 'Preparation of microemulsions and nanoemulsions by membrane emulsification'. Colloids and Surfaces A: Physicochemical and Engineering Aspects 579: 123709, 2019. DOI: 10.1016/j.colsurfa. 2019. 123709. (see p. 48)

[83] R. Farzad, S. Puttinger, S. Pirker and S. Schneiderbauer. 'Investigation of droplet size distribution for liquid-liquid emulsions in Taylor-Couette flows'. Journal of Dispersion Science and Technology 39: 250-258, 2018. DOI: 10.1080/01932691.2017.1312431. (see p. 48)

[84] S. Schultz, G. Wagner, K. Urban and J. Ulrich. 'High-Pressure Homogenization as a Process for Emulsion Formation'. Chemical Engineering 86 Technology 27: 361-368, 2004. DOI: 10.1002/ceat.200406111. (see p. 48)

[85] R.G. SweEt. 'High frequency recording with electrostatically deflected ink jets'. Review of Scientific Instruments 36: 131-136, 1965. DOI: 10.1063/1. 1719502. (see p. 48)

[86] N. Convery and N. Gadegaard. '30 Years of Microfluidics'. Micro and Nano Engineering 2: 76-91, 2019. DOI: 10.1016/j.mne.2019.01.003. (see p. 48)

[87] G.M. Whitesides. 'The origins and the future of microfluidics'. Nature 442: 368-373, 2006. DOI: 10.1038/nature05058. (see p. 48)

[88] P. Zhu and L. WANG. 'Passive and active droplet generation with microfluidics: a review'. Lab on a Chip 17: 34-75, 2017. DOI: 10.1039/c61c01018k. (see pp. 48, 50)

[89] T. Kawakatsu, Y. Kikuchi and M. Nakajima. 'Regular-sized cell creation in microchannel emulsification by visual microprocessing method'. Journal of the American Oil Chemists' Society 74: 317-321, 1997. DOI: 10.1007/ s11746-997-0143-8. (see p. 48) 
[90] K.C. van Dijke, G. Veldhuis, K. SchroËn and R.M. Boom. 'Simultaneous formation of many droplets in a single microfluidic droplet formation unit'. AIChE Journal 56: 833-836, 2009. DOI: 10.1002/aic.11990. (see p. 48)

[91] S. Sugiura, M. Nakajima, S. Iwamoto and M. Seki. 'Interfacial Tension Driven Monodispersed Droplet Formation from Microfabricated Channel Array'. Langmuir 17: 5562-5566, 2001. DOI: 10.1021/1a010342y. (see p. 48)

[92] G.T. Vladisavljević, I. Kobayashi and M. Nakajima. 'Production of uniform droplets using membrane, microchannel and microfluidic emulsification devices'. Microfluidics and Nanofluidics 13: 151-178, 2012. DOI: 10.1007/s10404-012-0948-0. (see p. 49)

[93] I. Kobayashi, K. Uemura and M. Nakajima. 'Formulation of monodisperse emulsions using submicron-channel arrays'. Colloids and Surfaces A: Physicochemical and Engineering Aspects 296: 285-289, 2007. DOI: $10.1016 / \mathrm{j}$. colsurfa.2006.09.015. (see p. 49)

[94] S. Sugiura, M. Nakajima and M. Seki. 'Preparation of monodispersed emulsion with large droplets using microchannel emulsification'. Journal of the American Oil Chemists' Society 79: 515-519, 2002. DOI: 10.1007/s11746002-0517-6. (see p. 49)

[95] L. Shui, E.S. Kooij, D. Wijnperlé, A. van den Berg and J.C.T. Eijkel. 'Liquid crystallography: 3D microdroplet arrangements using microfluidics'. Soft Matter 5: 2708, 2009. DOI: 10.1039/b908498c. (see pp. 49, 50)

[96] F. Malloggi, N. Pannacci, R. Attia, F. Monti, P. Mary, H. Willaime, P. Tabeling, B. Cabane and P. Poncet. 'Monodisperse Colloids Synthesized with Nanofluidic Technology'. Langmuir 26: 2369-2373, 2010. DOI: 10.1021/la9028047. (see p. 49)

[97] L. Shui, A. van den Berg and J.C.T. EiJkel. 'Scalable attoliter monodisperse droplet formation using multiphase nano-microfluidics'. Microfluidics and Nanofluidics 11: 87-92, 2011. DOI: 10.1007/s10404-011-0776-7. (see pp. 49, 50)

[98] E. Amstad, M. Chemama, M. Eggersdorfer, L.R. Arriaga, M.P. BrenNER and D.A. WEITZ. 'Robust scalable high throughput production of monodisperse drops'. Lab on a Chip 16: 4163-4172, 2016. DOI: 10.1039/ C6LC01075J. (see pp. 49, 67)

[99] A. Ofner, D.G. Moore, P.A. Rühs, P. Schwendimann, M. Eggersdorfer, E. Amstad, D.A. Weitz and A.R. Studart. 'High-Throughput Step Emulsification for the Production of Functional Materials Using a Glass Microfluidic Device'. Macromolecular Chemistry and Physics 218: 1600472, 2017. DOI: $10.1002 /$ macp. 201600472. (see p. 49) 
Bibliography

[100] P.G. Bolhuis and D.A. Kofke. 'Monte Carlo study of freezing of polydisperse hard spheres'. Physical Review E 54: 634-643, 1996. DOI: 10.1103/ physreve.54.634. (see p. 50)

[101] M. Meissner, J. Dong, J. Eggers, A.M. Seddon and C.P. Royall. 'Oilin-water microfluidics on the colloidal scale: new routes to self-assembly and glassy packings'. Soft Matter 13: 788-794, 2017. DOI: 10.1039/c6sm02390h. (see p. 50)

[102] R. Dangla, S.C. Kayi and C.N. Baroud. 'Droplet microfluidics driven by gradients of confinement'. Proceedings of the National Academy of Sciences 110: 853-858, 2013. DOI: 10.1073/pnas.1209186110. (see p. 50)

[103] A.S. Utada, E. Lorenceau, D.R. Link, P.D. Kaplan, H.A. Stone and D.A. Weitz. 'Monodisperse Double Emulsions Generated from a Microcapillary Device'. Science 308: 537-541, 2005. DOI: 10.1126/science.1109164. (see p. 51)

[104] M.L. Eggersdorfer, H. Seybold, A. Ofner, D.A. Weitz and A.R. STUDART. 'Wetting controls of droplet formation in step emulsification'. Proceedings of the National Academy of Sciences 115: 9479-9484, 2018. DOI: 10.1073/pnas. 1803644115. (see pp. 51-53, 62, 65)

[105] N. Mittal, C. Cohen, J. Bibette and N. Bremond. 'Dynamics of stepemulsification: From a single to a collection of emulsion droplet generators'. Physics of Fluids 26: 082109, 2014. DOI: 10.1063/1.4892949. (see p. 53)

[106] C.I. Zoldesi and A. ImHof. 'Synthesis of Monodisperse Colloidal Spheres, Capsules, and Microballoons by Emulsion Templating'. Advanced Materials 17: 924-928, 2005. DOI: 10.1002/adma.200401183. (see p. 55)

[107] S. PRAHL. miepython v1.3.0. https://github.com/scottprahl/miepython. 2019 (see p. 55)

[108] W.J. Wiscombe. Mie Scattering Calculations: Advances in Technique and Fast, Vector-speed Computer Codes. Tech. rep. National Center for Atmospheric Research, 1979. DOI: 10.5065/D6ZP4414 (see p. 55)

[109] C.F. Bohren and D.R. Huffman. In: 'Absorption and Scattering of Light by Small Particles'. Chap. A: Homogeneous Sphere, 477-482. John Wiley \& Sons, Ltd, 1998. DOI: 10.1002/9783527618156.app2. (see p. 55)

[110] J.-M. SchröDER and S. Wiegand. 'Experimental suppression of multiple scattering: Effect on dynamic and static scattering data'. Physical Chemistry Chemical Physics 2: 1493-1495, 2000. DOI: 10.1039/a909828c. (see p. 56)

[111] D.L. Camin, A.F. Forziati and F.D. Rossini. 'Physical Properties of nHexadecane, n-Decylcyclopentane, n-Decylcyclohexane, 1-Hexadecene and n-Decylbenzene'. The Journal of Physical Chemistry 58: 440-442, 1954. DOI: 10.1021/j150515a015. (see p. 56) 
[112] M.I. Bodnarchuk, M.V. Kovalenko, H. Groiss, R. Resel, M. Reissner, G. Hesser, R.T. Lechner, W. Steiner, F. Schäffler and W. Heiss. 'Exchange-Coupled Bimagnetic Wüstite/Metal Ferrite Core/Shell Nanocrystals: Size, Shape, and Compositional Control'. Small 5: 2247-2252, 2009. DOI: $10.1002 / \mathrm{smll} .200900635$. (see p. 56)

[113] B. de Nijs, S. Dussi, F. Smallenburg, J.D. Meeldijk, D.J. Groenendijk, L. Filion, A. Imhof, A. van BlaAderen and M. DiJkstra. 'Entropy-driven formation of large icosahedral colloidal clusters by spherical confinement'. Nature Materials 14: 56-60, 2014. DOI: 10.1038/nmat4072. (see p. 56)

[114] F. Franks. 'Solute-Water Interactions and the Solubility Behaviour of Longchain Paraffin Hydrocarbons'. Nature 210: 87-88, 1966. DOI: $10.1038 /$ 210087a0. (see p. 62)

[115] J. Gaitzsch, X. Huang and B. Voit. 'Engineering Functional Polymer Capsules toward Smart Nanoreactors'. Chemical Reviews 116: 1053-1093, 2015. DOI: 10.1021/acs. chemrev.5b00241. (see p. 62)

[116] O.D. Velev, K. Furusawa and K. Nagayama. 'Assembly of Latex Particles by Using Emulsion Droplets as Templates. 1. Microstructured Hollow Spheres'. Langmuir 12: 2374-2384, 1996. DOI: 10.1021/la9506786. (see p. 67)

[117] N. Schuergers, T. Lenn, R. Kampmann, M.V. Meissner, T. Esteves, M. Temerinac-Ott, J.G. Korvink, A.R. Lowe, C.W. Mullineaux and A. WILDE. 'Cyanobacteria use micro-optics to sense light direction'. eLife 5: 12620, 2016. DOI: 10.7554/elife.12620. (see p. 74)

[118] E. Hecht. Optics. 4th ed. Addison-Wesley, 2002. (see p. 74)

[119] E. AbBe and H. Fripp. 'A contribution to the theory of the microscope, and the nature of microscopic vision.' Proceedings of the Bristol Naturalists' Society, New Series 1: 200-58, 1874. (see p. 74)

[120] S. Inoué and R. Oldenbourg. 'Microscopes'. In: Handbook of Optics, Volume 2. Ed. by M. BAss. 2nded. Vol. 2. New York: McGraw-Hill Professional, 1994. Chap. 17, 1-52. (see p. 75)

[121] M. Minsky. 'Microscopy apparatus'. U.S. pat. 3013467. 1961 (see p. 75)

[122] S. InOUÉ. 'Foundations of Confocal Scanned Imaging in Light Microscopy'. In: Handbook Of Biological Confocal Microscopy. Ed. by J.B. PAWLEY. 3rd ed. Boston, MA: Springer US, 2006. 1-19. DOI: 10.1007/978-0-387-45524-2_1. (see p. 76 )

[123] B.D. Leahy, N.Y.C. Lin and I. Cohen. 'Quantitative light microscopy of dense suspensions: Colloid science at the next decimal place'. Current Opinion in Colloid and Interface Science 34: 32-46, 2018. DOI: 10.1016/j. cocis.2018.03.002. (see p. 76) 
Bibliography

[124] S.W. Hell and J. Wichmann. 'Breaking the diffraction resolution limit by stimulated emission: stimulated-emission-depletion fluorescence microscopy'. Optics Letters 19: 780-2, 1994. DOI: 10.1364/OL.19.000780. (see p. 76)

[125] S.W. HeLL et al. 'The 2015 super-resolution microscopy roadmap'. Journal of Physics D: Applied Physics 48: 443001, 2015. DOI: $10.1088 / 0022-$ 3727/48/44/443001. (see p. 76)

[126] P. Bianchini, C. Peres, M. Oneto, S. Galiani, G. Vicidomini and A. DiAspro. 'STED nanoscopy: a glimpse into the future'. Cell and Tissue Research 360: 143-150, 2015. DOI: 10.1007/s00441-015-2146-3. (see p. 76)

[127] S.W. Hell, K.I. Willig, M. Dyba, S. Jakobs, L. Kastrup and V. WestPHAL. 'Nanoscale Resolution with Focused Light: Stimulated Emission Depletion and Other Reversible Saturable Optical Fluorescence Transitions Microscopy Concepts'. In: Handbook Of Biological Confocal Microscopy. Ed. by J.B. PAWLEY. 3rd ed. Boston, MA: Springer US, 2006. Chap. 31, 571-579. DOI: $10.1007 / 978-0-387-45524-2 \_31$. (see p. 76)

[128] T.A. Klar, S. Jakobs, M. Dyba, A. Egner and S.W. Hell. 'Fluorescence microscopy with diffraction resolution barrier broken by stimulated emission.' Proceedings of the National Academy of Sciences of the United States of America 97: 8206-10, 2000. (see pp. 76, 89)

[129] M. Reuss. 'Simpler S ted Setups'. PhD thesis. Heidelberg University, 2010. (see pp. 76, 89)

[130] H. Blom and J. Widengren. 'Stimulated Emission Depletion Microscopy'. Chemical Reviews 117: 7377-427, 2017. DOI: 10 . 1021/acs . chemrev . 6b00653. (see pp. 76, 88)

[131] G. Donnert, J. Keller, R. Medda, M. Alexandra Andrei, S.O. Rizzoli, R. Lührmann, R. Jahn, C. Eggeling and S.W. Hell. 'Macromolecularscale resolution in biological fluorescence microscopy'. Proceedings of the National Academy of Sciences 103: 11440-5, 2006. DOI: 10.1073/pnas . 0604965103. (see p. 76)

[132] K.I. Willig, S.O. Rizzoli, V. Westphal, R. Jahn and S.W. Hell. 'S ted microscopy reveals that synaptotagmin remains clustered after synaptic vesicle exocytosis'. Nature 440: 935-9, 2006. DOI: 10.1038/nature04592. (see p. 76)

[133] K.I. Willig, J. Keller, M. Bossi and S.W. Hell. 'Sted microscopy resolves nanoparticle assemblies'. New Journal of Physics 8: 106, 2006. DOI: 10.1088/1367-2630/8/6/106. (see p. 76)

[134] L. Meyer, D. Wildanger, R. Medda, A. Punge, S.O. Rizzoli, G. DonNERT and S.W. HeLl. 'Dual-Color S TEd Microscopy at 30-nm Focal-Plane Resolution'. Small 4: 1095-100, 2008. DOI: 10.1002/smll.200800055. (see p. 76) 
[135] S. Hell, G. Reiner, C. Cremer and E.H.K. Stelzer. 'Aberrations in confocal fluorescence microscopy induced by mismatches in refractive index'. Journal of microscopy 169: 391-405, 1993. (see pp. 76, 89, 95)

[136] S. Deng, L. Liu, Y. Cheng, R. Li and Z. Xu. 'Investigation of the influence of the aberration induced by a plane interface on STED microscopy'. Optics Express 17: 1714, 2009. DOI: 10.1364/oe.17.001714. (see pp. 77, 89)

[137] S. Deng, L. Liu, Y. Cheng, R. Li and Z. Xu. 'Effects of primary aberrations on the fluorescence depletion patterns of STED microscopy'. Optics Express 18: 1657, 2010. DOI: 10.1364/oe.18.001657. (see p. 77)

[138] B.R. Patton, D. Burke, D. Owald, T.J. Gould, J. Bewersdorf and M.J. Bоотн. 'Three-dimensional S TED microscopy of aberrating tissue using dual adaptive optics'. Optics Express 24: 8862, 2016. DOI: 10.1364/OE. 24. 008862. (see pp. 77,90 )

[139] G. Vicidomini, P. Bianchini and A. Diaspro. 'S TED super-resolved microscopy'. Nature Methods 15: 173-82, 2018. DOI: 10.1038/nmeth.4593. (see p. 77$)$

[140] N.T. Urban, K.I. Willig, S.W. Hell and U.V. NäGerl. 'S ted nanoscopy of actin dynamics in synapses deep inside living brain slices'. Biophysical Journal 101: 1277-84, 2011. DOI: 10.1016/j.bpj.2011.07.027. (see p. 77)

[141] T.J. Gould, D. Burke, J. Bewersdorf and M.J. Booth. 'Adaptive optics enables 3D STED microscopy in aberrating specimens'. Optics Express 20: 20998, 2012. DOI: 10.1364/oe.20.020998. (see p. 77)

[142] P.J. Shaw. 'Comparison of Widefield/Deconvolution and Confocal Microscopy for Three-Dimensional Imaging'. In: Handbook Of Biological Confocal Microscopy. Ed. by J.B. PAWLey. 3rded. Boston, MA: Springer US, 2006. Chap. 23, 453-467. DOI: 10.1007/978-0-387-45524-2_23. (see p. 77)

[143] M.B. Cannell, A. McMorland and C. Soeller. 'Image Enhancement by Deconvolution'. In: Handbook Of Biological Confocal Microscopy. Ed. by J.B. Pawley. 3rded. Boston, MA: Springer US, 2006. Chap. 25, 488-500. DOI: $10.1007 / 978-0-387-45524-2 \_25$. (see pp. 78, 80,81)

[144] H. Kirshner, F. Aguet, D. Sage and M. Unser. '3-D ps F fitting for fluorescence microscopy: Implementation and localization application'. Journal of Microscopy 249: 13-25, 2013. DOI: 10.1111/j.1365-2818.2012.03675.x. (see p. 79)

[145] T.J. Holmes, D. Biggs and A. Abu-Tarif. 'Blind Deconvolution'. In: Handbook Of Biological Confocal Microscopy. Ed. by J.B. PAWLEY. 3rded. Boston, MA: Springer US, 2006. Chap. 24, 468-487. DOI: 10.1007/978-0387-45524-2_24. (see p. 78)

[146] R.C. Gonzalez and R.E. Woods. In: 'Digital image processing'. Chap. 5, 311-93. Upper Saddle River, N.J.: Prentice Hall, 2008. (see p. 78) 
[147] P.H. van Cittert. 'Zum Einfluß der Spaltbreite auf die Intensitätsverteilung in Spektrallinien'. Zeitschrift für Physik 65: 547-563, 1930. DOI: $10.1007 /$ bf01397051. (see p. 80)

[148] P.A. Jansson, R.H. Hunt and E.K. Plyler. 'Resolution Enhancement of Spectra'. Journal of the Optical Society of America 60: 596-9, 1970. DOI: 10.1364/josa.60.000596. (see p. 80)

[149] W.H. Richardson. 'Bayesian-based iterative method of image restoration'. Journal of the Optical Society of America 62: 55-9, 1972. DOI: 10.1364/ JOSA.62.000055. (see p. 81)

[150] L.B. LuCY. 'An iterative technique for the rectification of observed distributions'. Astronomical Journal 79: 745-54, 1974. (see p. 81)

[151] D. Sage, L. Donati, F. Soulez, D. Fortun, G. Schmit, A. Seitz, R. Guiet, C. Vonesch and M. Unser. 'DeconvolutionLab2: An open-source software for deconvolution microscopy'. Methods 115: 28-41, 2017. DOI: 10.1016/j.ymeth.2016.12.015. (see pp. 81, 87, 89, 109)

[152] H. Trussell and B. Hunt. 'Image restoration of space-variant blurs by sectioned methods'. IEEE Transactions on Acoustics, Speech, and Signal Processing 26: 608-609, 1978. DOI: 10.1109/TASSP.1978.1163161. (see p. 81)

[153] C. Preza and J.-A. Conchello. 'Image estimation accounting for pointspread function depth variation in three-dimensional fluorescence microscopy'. Three-Dimensional and Multidimensional Microscopy: Image Acquisition and Processing X 4964: 135-42, 2003. DOI: 10.1117/12.481116. (see p. 81)

[154] Y. Chen, M. Chen, L. Zhu, J.Y. Wu, S. Du and Y. Li. 'Measure and model a 3-D space-variant P SF for fluorescence microscopy image deblurring'. Optics Express 26: 14375, 2018. DOI: 10.1364/OE.26.014375. (see p. 82)

[155] S.F. Gibson and F. LANNI. 'Experimental test of an analytical model of aberration in an oil-immersion objective lens used in three-dimensional light microscopy'. Journal of the Optical Society of America A 8: 1601-13, 1991. DOI: $10.1364 /$ josaa.8.001601. (see p. 82)

[156] R.W. Cole, T. Jinadasa and C.M. Brown. 'Measuring and interpreting point spread functions to determine confocal microscope resolution and ensure quality control'. Nature Protocols 6: 1929-41, 2011. DOI: 10.1038/nprot. 2011.407. (see p. 82)

[157] A. Diaspro, F. Federici and M. Robello. 'Influence of refractive-index mismatch in high-resolution three-dimensional confocal microscopy'. Applied Optics 41: 685-90, 2002. DOI: 10.1364/ao.41.000685. (see p. 82)

[158] J.W. Shaevitz and D.A. Fletcher. 'Enhanced three-dimensional deconvolution microscopy using a measured depth-varying point-spread function'. Journal of the Optical Society of America A 24: 2622-7, 2007. DOI: 10.1364/ JOSAA.24.002622. (see p. 82) 
[159] J.J. Schmied, R. Dijkstra, M.B. Scheible, G.M.R. De Luca and J.J. SIEBER. Measuring the 3D STED-PSF with a new type of fluorescent beads. Leica Microsystems. Sept. 2016. (see p. 82)

[160] E.B. van der Wee, J. Fokkema, C.L. Kennedy, M. de Pozo-Puig, D.A.M. DE Winter, P.N.A. Speets, H.C. Gerritsen and A. van BLAADEREN. ' $3 \mathrm{D}$ test sample for the calibration and quality control of super-resolution and confocal microscopes'. Submitted. (see pp. 82, 84)

[161] H. EL-KASheF. 'The necessary requirements imposed on polar dielectric laser dye solvents'. Physica B: Condensed Matter 279: 295-301, 2000. DOI: 10.1016/S0921-4526(01)00642-1. (see p. 83)

[162] Y. Sambuy, I. De Angelis, G. Ranaldi, M.L. Scarino, A. Stammati and F. ZuCCO. 'The Caco-2 cell line as a model of the intestinal barrier: influence of cell and culture-related factors on Caco-2 cell functional characteristics'. Cell Biology and Toxicology 21: 1-26, 2005. DOI: 10.1007/s10565-0050085-6. (see p. 84)

[163] C.K. Carniglia, L. Mandel and K.H. Drexhage. 'Absorption and Emission of Evanescent Photons'. Journal of the Optical Society of America 62: 479-86, 1972. DOI: 10.1364/josa.62.000479. (see p. 84)

[164] H.T.M. van Der VoorT and K.C. Strasters. 'Restoration of confocal images for quantitative image analysis'. Journal of Microscopy 178: 165-81, 1995. (see p. 87)

[165] LeICA. 'Glycerol Objective'. Leica Confocal Application Letter, 2004. (see p. 93)

[166] S.W. Crawley, M.S. Mooseker and M.J. Tyska. 'Shaping the intestinal brush border'. Journal of Cell Biology 207: 441-51, 2014. DOI: 10.1083/ jcb.201407015. (see p. 101)

[167] Precision Abbe Refractometer $3 T$ Instruction Manual (Cat. No. 1230). Atago, Tokyo, 1997. (see p. 113)

[168] F.A. Jenkins and H.E. White. Fundamentals of optics. 3rded. McGrawHill, 1957. (see p. 112)

[169] B.V. Derjaguin, G. Sidorenkov, E. Zubashchenko and E. Kiseleva. 'Kinetic Phenomena in the boundary layers of liquids 1. The capillary osmosis'. Progress in Surface Science 43: 138-152, 1993. DOI: 10.1016/0079-6816(93) 90023-o. Trans. of Kolloidnyi Zhurnal 9: 335-347, 1947. (see pp. 116, 139)

[170] B.V. Derjaguin, S.S. Dukhin and A.A. Korotkova. 'Diffusiophoresis in electrolyte solutions and its role in the mechanism of the formation of films from caoutchouc latexes by the ionic deposition method'. Progress in Surface Science 43: 153-158, 1993. DOI: 10.1016/0079-6816(93)90024-p. (see pp. 116, 120, 121) 
Bibliography

[171] B.V. Deryagin, Z.R. Ul'berg, G.L. Dvornichenko and S.S. Dukhin. 'Use of surface chemical reactions to form diffusiophoretic coatings'. Colloid Journal of the USSR 42: Consultants Bureau, New York, 711-714, 1980. (see p. 116)

[172] S.S. Dukhin, Z.R. Ul'berg, G.L. Dvornichenko and B.V. Deryagin. 'Diffusiophoresis in electrolyte solutions and its application to the formation of surface coatings'. Bulletin of the Academy of Sciences of the USSR Division of Chemical Science 31: 1535-1544, 1982. DOI: 10.1007/bf00956888. (see p. 116)

[173] M.M.-J. LiN and D.C. PrIEve. 'Electromigration of latex induced by a salt gradient'. Journal of Colloid and Interface Science 95: 327-339, 1983. DOI: 10.1016/0021-9797(83)90192-3. (see p. 116)

[174] S.S. Dukhin and B.V. Derjaguin. Electrokinetic phenomena. Ed. by E. MatiJević. Vol. 7. Surface and Colloid Science. Wiley, 1974. (see pp. 116, 119-121, 139)

[175] B.V. Deruaguin, S.S. Dukhin and V.N. Shilov. 'Kinetic aspects of electrochemistry of disperse systems. Part I. Introduction'. Advances in Colloid and Interface Science 13: 141-152, 1980. DOI: 10.1016/0001-8686(80)87004-7. (see p. 116)

[176] J.L. Anderson. 'Motion of a charged particle in a gradient of electrolyte'. PhysicoChemical Hydrodynamics 1: 51-56, 1980. (see pp. 116, 121)

[177] J.L. Anderson, M.E. Lowell and D.C. Prieve. 'Motion of a particle generated by chemical gradients Part 1. Non-electrolytes'. Journal of Fluid Mechanics 117: 107-121, 1982. DOI: 10.1017/s0022112082001542. (see pp. 116, 119, 121)

[178] D.C. Prieve, J.L. Anderson, J.P. Ebel and M.E. Lowell. 'Motion of a particle generated by chemical gradients. Part 2. Electrolytes'. Journal of Fluid Mechanics 148: 247-269, 1984. DOI: 10.1017/s0022112084002330. (see pp. 116, 120, 121, 139)

[179] D.C. PRIEVE and R. Roman. 'Diffusiophoresis of a rigid sphere through a viscous electrolyte solution'. Journal of the Chemical Society, Faraday Transactions 2 83: 1287, 1987. DOI: 10.1039/f29878301287. (see pp. 116, $117,120-122)$

[180] S.S. Dukhin. 'Non-equilibrium electric surface phenomena'. Advances in Colloid and Interface Science 44: 1-134, 1993. DOI: 10.1016/0001-8686(93) 80021-3. (see p. 116)

[181] J.L. Moran and J.D. Posner. 'Phoretic Self-Propulsion'. Annual Review of Fluid Mechanics 49: 511-540, 2017. DOI: 10.1146/annurev-fluid-122414034456. (see p. 116) 
[182] B.V. Deryagin, S.S. Dukhin, Z.R. Ul'berg and T.V. Kuznetsova. 'Microscopic method for investigating diffusiophoresis'. Colloid Journal of the USSR 42: Consultants Bureau, New York, 382-385, 1980. (see p. 116)

[183] W.J. Lechnick and J.A. Shaeiwitz. 'Measurement of diffusiophoresis in liquids'. Journal of Colloid and Interface Science 102: 71-87, 1984. DOI: 10.1016/0021-9797(84)90201-7. (see p. 117)

[184] J.P. Ebel, J.L. Anderson and D.C. Prieve. 'Diffusiophoresis of latex particles in electrolyte gradients'. Langmuir 4: 396-406, 1988. DOI: 10. 1021/la00080a024. (see p. 117)

[185] D.M. HiLl. Latex Dipping: Science and Technology. 2nd ed. De Gruyter, 2019. (see p. 117)

[186] P.O. Staffeld and J.A. Quinn. 'Diffusion-induced banding of colloid particles via diffusiophoresis: 1. Electrolytes'. Journal of Colloid and Interface Science 130: 69-87, 1989. DOI: 10 .1016/0021-9797(89) 90079-9. (see pp. 117, 130)

[187] P.O. Staffeld and J.A. Quinn. 'Diffusion-induced banding of colloid particles via diffusiophoresis: 2. Non-Electrolytes'. Journal of Colloid and Interface Science 130: 88-100, 1989. DOI: 10.1016/0021-9797(89)90080-5. (see p. 117)

[188] D. Qin, Y. XiA and G.M. Whitesides. 'Soft lithography for micro- and nanoscale patterning'. Nature Protocols 5: 491-502, 2010. DOI: 10.1038/ nprot.2009.234. (see pp. 117, 126)

[189] B. Abécassis, C. Cottin-Bizonne, C. Ybert, A. Ajdari and L. Bocquet. 'Boosting migration of large particles by solute contrasts'. Nature Materials 7: 785-789, 2008. DOI: 10.1038/nmat2254. (see pp. 117, 118, 123)

[190] B. Abécassis, C. Cottin-Bizonne, C. Ybert, A. Ajdari and L. Bocquet. 'Osmotic manipulation of particles for microfluidic applications'. New Journal of Physics 11: 075022, 2009. DOI: 10.1088/1367-2630/11/7/075022. (see pp. 117, 118, 123-125, 130)

[191] J.S. Paustian, C.D. Angulo, R. Nery-Azevedo, N. Shi, A.I. AbdelFATTAH and T.M. SQuires. 'Direct Measurements of Colloidal Solvophoresis under Imposed Solvent and Solute Gradients'. Langmuir 31: 4402-4410, 2015. DOI: 10.1021/acs.langmuir.5b00300. (see pp. 118, 123)

[192] R. Nery-Azevedo, A. Banerjee and T.M. Squires. 'Diffusiophoresis in Ionic Surfactant Gradients'. Langmuir 33: 9694-9702, 2017. DOI: 10.1021/ acs. langmuir.7b01094. (see p. 118)

[193] S.T. Wereley and C.D. Meinhart. 'Recent Advances in Micro-Particle Image Velocimetry'. Annual Review of Fluid Mechanics 42: 557-576, 2010. DOI: 10.1146/annurev-fluid-121108-145427. (see p. 118) 
Bibliography

[194] T. Dracos. 'Particle Tracking Velocimetry (P TV)'. In: Three-Dimensional Velocity and Vorticity Measuring and Image Analysis Techniques. Springer Netherlands, 1996. 155-160. DOI: 10.1007/978-94-015-8727-3_7. (see p. 118)

[195] P.G. Moerman. 'Dynamics of active droplets and freely jointed colloidal trimers'. PhD thesis. Utrecht University, 2019. (see p. 118)

[196] A. Visan and R.G.H. LAmmertink. 'Reaction induced diffusio-phoresis of ordinary catalytic particles'. Reaction Chemistry \& Engineering 4: 14391446, 2019. DOI: 10.1039/c9re00103d. (see pp. 118, 140)

[197] D. Velegol, A. Garg, R. Guha, A. Kar and M. Kumar. 'Origins of concentration gradients for diffusiophoresis'. Soft Matter 12: 4686-4703, 2016. DOI: $10.1039 / \mathrm{c} 6 \mathrm{sm} 00052$ e. (see pp. 119, 123)

[198] S. Marbach, H. Yoshida and L. Bocquet. 'Local and global force balance for diffusiophoretic transport'. Journal of Fluid Mechanics 892: 2020. DOI: 10.1017/jfm.2020.137. (see p. 120)

[199] R.J. Hunter. Foundations of Colloid Science. 2nd ed. Oxford University Press, 2001. Chap. 7: Electrified Interfaces: The Electrical Double Layer, 304-372. (see p. 120)

[200] R.J. Hunter. Foundations of Colloid Science. 2nd ed. Oxford University Press, 2001. Chap. 8: Electrokinetics and the zeta potential, 374-433. (see pp. 121, 123)

[201] J.M.G. Barthel, H. Krienke and W. Kunz. Physical Chemistry of Electrolyte Solutions. Topics in Physical Chemistry 5. Steinkopff/Springer, 1998. (see p. 121)

[202] H. Ohshima. 'A Simple Expression for Henry's Function for the Retardation Effect in Electrophoresis of Spherical Colloidal Particles'. Journal of Colloid and Interface Science 168: 269-271, 1994. DOI: 10.1006/jcis.1994.1419. (see p. 123)

[203] R.W. O'Brien and L.R. White. 'Electrophoretic mobility of a spherical colloidal particle'. Journal of the Chemical Society, Faraday Transactions 2 74: 1607, 1978. DOI: 10.1039/f29787401607. (see p. 123)

[204] G.K. BAtchelor. 'Flow of a Uniform Incompressible Viscous Fluid'. In: An Introduction to Fluid Dynamics. Cambridge University Press, Feb. 2000. Chap. 4, 174-263. DOI: 10.1017/cbo9780511800955.006. (see p. 125)

[205] J.-B. SAlmon and A. AJdARI. 'Transverse transport of solutes between coflowing pressure-driven streams for microfluidic studies of diffusion/reaction processes'. Journal of Applied Physics 101: 074902, 2007. DOI: 10.1063/1. 2714773. (see p. 125)

[206] R.W. Balluffi, S.M. Allen and W.C. Carter. In: 'The Diffusion Equation'. Chap. 4, 77-97. John Wiley \& Sons, Inc., Dec. 2005. DOI: $10.1002 /$ 0471749311.ch4. (see p. 125) 
[207] H. Chanson. 'Diffusion: basic theory'. In: Environmental Hydraulics of Open Channel Flows. Elsevier, 2004. 65-74. DOI: 10.1016/b978-0750661652.50037-0. (see p. 125)

[208] K.G. Romanchuk. 'Fluorescein. Physicochemical factors affecting its fluorescence'. Survey of Ophthalmology 26: 269-283, 1982. DOI: 10.1016/00396257(82)90163-1. (see p. 128)

[209] C. van der Wel, R.K. Bhan, R.W. Verweij, H.C. Frijters, Z. Gong, A.D. Hollingsworth, S. Sacanna and D.J. Kraft. 'Preparation of Colloidal Organosilica Spheres through Spontaneous Emulsification'. Langmuir 33: 8174-8180, 2017. DOI: 10.1021/acs.langmuir.7b01398. (see p. 136)

[210] T. Casalini, M. Salvalaglio, G. Perale, M. Masi and C. Cavallotti. 'Diffusion and Aggregation of Sodium Fluorescein in Aqueous Solutions'. The Journal of Physical Chemistry B 115: 12896-12904, 2011. DOI: 10.1021/ jp207459k. (see p. 136)

[211] P. VANÝSEK. 'Ionic conductivity and diffusion at infinite dilution'. In: $C R C$ Handbook of Chemistry and Physics. Ed. by J.R. Rumble. 101sted. CRC Press, 2020. (see p. 136)

[212] A. AJdAri and L. Bocquet. 'Giant Amplification of Interfacially Driven Transport by Hydrodynamic Slip: Diffusio-Osmosis and Beyond'. Physical Review Letters 96: 2006. DOI: 10.1103/physrevlett.96.186102. (see p. 139)

[213] Z. Almutairi, C.L. Ren and L. Simon. 'Evaluation of polydimethylsiloxane (PDMS) surface modification approaches for microfluidic applications'. Colloids and Surfaces A: Physicochemical and Engineering Aspects 415: 406412, 2012. DOI: 10.1016/j.colsurfa.2012.10.008. (see p. 139)

[214] G.T. Roman, T. Hlaus, K.J. Bass, T.G. Seelhammer and C.T. CulBERTSOn. 'Sol-Gel Modified Poly(dimethylsiloxane) Microfluidic Devices with High Electroosmotic Mobilities and Hydrophilic Channel Wall Characteristics'. Analytical Chemistry 77: 1414-1422, 2005. DOI: 10.1021/ac048811z. (see p. 139) 



\section{Summary for a broad audience}

Water and oil do not mix. Therefore if we pour cooking oil into a glass of water, it will form a separate oil region, floating atop the water. It is possible though to spread the oil through the water, and without breaking our intuitive understanding that they do not mix. The trick is to make and maintain very small portions of the substance to be dispersed. The combination is then known as a colloidal dispersion. One example is milk, which contains tiny oil droplets in a sea of water. Thus the oil and water are still in separate regions, but now there are billions of small oil regions spread throughout one big water one. Such a dispersion is different from a true solution. Ethanol, for example, cannot form a colloidal system in water because the two substances mix completely, making a single region of ethanol-water solution.

The two components need not be liquids to form a colloidal dispersion. Toothpaste contains solid particles which participate in microscopic sandblasting of your teeth. More tasty are the solids swirling around in a cup of coffee and the lethargic fragments of fruit in tomato ketchup. A gas may also be involved such as in fog, which is water droplets dispersed in air. The solid particles or liquid droplets in all of these are too small to be seen with the naked eye. In fact, with sizes around 1 micrometre $(\mu \mathrm{m})$, they are about 100 times smaller than the breadth of a hair but still 10,000 times larger than atoms. Particles of this size are able to stay dispersed in the continuous liquid for a long time: a glass of milk left on the counter will remain opaque while a mixture of sand and water quickly separates. Another important property of colloidal systems is the very large surface area of contact between the two substances: surface area increases whenever an object is broken down into smaller pieces. In addition to their occurrence in nature and everyday life, the special properties of colloidal particles are often put to work in industrial processes and fundamental research.

The modern world has high demands for machinery and some of these are met by colloidal matter in the form of paints and lubricants. Colloid science also has a role to play in the processing of minerals and manufacture of ceramics.

In research laboratories, colloids may be studied for industrial applications but they have further utility as models for much smaller entities like atoms or molecules. A model should share at least some properties with the real system, but should also be easier to observe. For example, oranges can be used to 
model atoms and test theories about the most efficient packing of balls into boxes. Because oranges have only a few properties in common with atoms (most importantly being more or less spherical), the atomic theories which can be tested are quite limited. Colloidal particles have more properties in common with atoms and molecules and therefore can be used as a better model. Objects which cannot be seen would seem to be unfit to serve as a model, when one might just as well use the invisible atoms themselves. The difference is that colloids can be observed with any high school microscope, which is convenient enough to make the model useful.

The abilities to create, observe and manipulate colloidal particles are central to colloid science. The work of this thesis was to make a contribution to each of these three pillars of the discipline, to sharpen the tools which give us these abilities and to understand what is happening at the cutting edge.

Presented first is the 'creation' (synthesis) part of the thesis. In Chapter 2, we improved methods of making spherical silica (glass) particles. These can be used as a model for atomic behaviour. The particle sizes can be controlled by adding layers (like growing a snowball) until a desired size is reached. The challenges lie in ensuring that all the particles are the same size and that they stay separate during growth. Otherwise the result will not be uniform spheres but a hodgepodge of snowman shaped particles. These problems are most prevalent for smaller particles (e.g. $<0.5 \mu \mathrm{m})$. These smaller particles are less affected by gravity and so behave more like atoms (and less like oranges). With recent improvements in microscopy, smaller particles can be imaged. In this chapter, we overcame the synthetic challenges associated with making uniform small particles, in order to take advantage of our new ability to observe this better model system.

Chapter 3 is also concerned with creation of colloidal particles, but in this case liquid droplets. These are also difficult to make uniform in diameter, especially in the colloidal size range. One solution is to make each droplet one at a time using something like a $\mu \mathrm{m}$ sized dripping tap. Fluid handling at this small scale is called 'microfluidics'. This one at a time method is very slow compared to other methods of droplet production, so in Chapter 3 we investigated a method involving 5000 such 'taps' dripping in parallel. In this way we were able to speed up the production of uniformly sized colloidal droplets.

In the 'observation' part of the thesis, Chapter 4 is concerned with testing imaging capability of a modern optical microscope. For this measurement, one should use an object with a size close to the smallest thing that can be resolved. Such measurements allow improved restoration of images in post processing. As an example, imagine a camera which takes photos which are always blurred vertically (maybe the sensor/film is loose and moves when the shutter button 
is pressed). If you take a photo of a small/far away source of light (e.g. a star or a pinhole), it will appear as a vertical line, when we know it should look like a dot. Based on how stretched dot has become, we know how much blurring is taking place. If we take another photo of a face, the blur will render it almost unrecognisable. However, if we imagine the portrait to be made up of many of the dots that we measured, we can apply a reverse process and undo some of the blurring. This allows more detail to be seen in the restored photo, although some information is irrecoverably lost.

The microscope in question is one which is able to make $3 \mathrm{D}$ volume images by taking a picture of one slice at a time, similar to how a medical $\mathrm{CT}$ scanner looks inside a patient. Microscopes are usually calibrated with a flat test target but this can only measure two out of three dimensions in a $3 \mathrm{D}$ microscope. Therefore in this chapter, we developed a thick test sample with the required small objects (fluorescent silica spheres) distributed in $3 \mathrm{D}$.

Finally, in Chapter 5, we investigated a way of manipulating colloidal particles. Particles respond to differences in salt concentration by moving towards or away from areas of high concentration. Thus by controlling salt concentrations in their environment, we can change their speed and direction of movement. The experimental procedure involved particles flowing along a microfluidic channel with different salt concentration in the centre and at the sides.

To illustrate this, consider a street with a crowd exiting a stadium at one end. People will not respond much to salt on the ground so instead we can study their response to chewing gum stuck to the street. We put a lot of chewing gum close to the outer edges and none in the middle. Since nobody likes to walk on sticky gum, the people who began at the sides of the streaming crowd will start moving towards the middle of the street. The particles do something similar, although they usually move toward the region of high salt.

This phenomenon has previously been studied by examining the averaged behaviour of many particles. For our analogy, this is akin to studying the people using only the overlapping footprints left when the crowd is gone. From these, the tendency to move into the middle of the street is clear, and the approximate speed at which people do so may be inferred from how far from the stadium doors the footprints become focussed in the middle. Such a study would struggle to test a detailed theory predicting that the people move to the middle at different speeds, depending on how much chewing gum is currently under their feet. In this work, we zoomed in and analysed videos of individual particles and were able to confirm the fine details of a comparable theoretical prediction. 



\section{Samenvatting voor een breed publiek}

Water en olie mengen niet. Daarom krijgen we twee aparte delen als we olie in een glas water schenken: een gedeelte met alleen water en een ander deel met alleen olie. Echter is het mogelijk om de olie door het water te verspreiden én ons begrip te houden dat ze niet mengen. De truc is om hele kleine druppeltjes te maken. De combinatie heet dan een colloïdaal mengsel. Melk is een voorbeeld hiervan. Het bevat kleine oliedruppeltjes in een zee van water. De olie en het water bevinden zich dus nog steeds in aparte gebieden, maar nu zijn er miljarden oliegebiedjes verspreid door een groot watergebied. Dit soort mengsels zijn anders dan een oplossing. Ethanol bijvoorbeeld vormt nooit een colloïdaal mengsel in water want de twee stoffen mengen helemaal door elkaar. De resultaat is dan één gebied dat een ethanol-water oplossing bevat.

De twee stoffen hoeven niet vloeibaar te zijn om een colloïdaal mengsel te vormen. Tandpasta bevat wel water maar ook vaste deeltjes die door een soort miniatuur zandstralen je tanden schoon houden. Lekkerdere deeltjes die je regelmatig tegenkomt zijn de vaste stoffen die rondwervelen in een kopje koffie en de fruitdeeltjes die in tomatenketchup zitten. Een gas kan ook mee doen, zoals in de nevel, die waterdruppeltjes verspreid door lucht is. De vaste deeltjes of vloeibare druppeltjes zijn te klein om met het blote oog te zien. Met groottes van ongeveer 1 micrometer $(\mu \mathrm{m})$ zijn ze 10.000 keer groter dan atomen en 100 keer kleiner dan de breedte van een haar. Deeltjes van deze maat kunnen voor een lange tijd verspreid blijven door een andere stof: een glas melk op tafel blijft ondoorzichtig terwijl een mengsel van zand en water zich snel scheidt. Een andere belangrijke eigenschap van colloïdale systemen is het grote raakvlak tussen de twee stoffen. Dit komt doordat het oppervlak vergroot als een voorwerp tot kleinere stukjes afgebroken wordt. Naast hun voorkomen in de natuur en in het alledaagse leven, worden de bijzondere eigenschappen van colloïdaal materie vaak aan het werk gezet zowel in industriële processen als fundamenteel onderzoek.

De moderne wereld heeft veel hoge eisen voor machines en aan sommige hiervan wordt voldaan dankzij colloïdaal materie, bijvoorbeeld in de vorm van verven en smeermiddelen. De colloïdchemie heeft ook een rol in het verwerken 
van mineralen en in de productie van keramiek.

In onderzoekslaboratoria worden colloïden bestudeerd voor deze industriële toepassingen, maar ze worden ook vaak gebruikt als modellen voor veel kleinere entiteiten, zoals atomen of moleculen. Een model moet tenminste wat eigenschappen gemeen met het echte systeem en moet ook makkelijker te observeren zijn. Bijvoorbeeld, sinaasappels kunnen gebruikt worden om atomen na te bootsen, en theorieën van efficiënte bolstapeling te testen. Omdat sinaasappels maar een paar eigenschappen gemeen met atomen hebben (zoals bolvormig zijn), zijn de atomistische theorieën die getest kunnen worden beperkt. Colloïdale deeltjes hebben meer eigenschappen gemeen met atomen en moleculen en vormen daardoor een beter modelsysteem. Onzichtbare dingen klinken misschien als onhandige model. Echter zijn collö̈den makkelijk om te zien met de meest simpele microscoop en dit gemak maakt het model nuttig.

Het creëren, observeren en manipuleren van deeltjes staat centraal in de colloïdchemie. Het werk van dit proefschrift is een bijdrage aan elk van deze drie pijlers van het vakgebied door de gereedschappen die ermee geassocieerd worden te verfijnen en de beperkingen ervan te begrijpen.

Het proefschrift start met het 'creëren' (synthese) gedeelte. In Hoofdstuk 2 verbeterden we methoden voor het maken van bolvormige glasdeeltjes. Deze deeltjes kunnen gebruikt worden in een model dat het gedrag van atomen nabootst. Controle over de grootte van deze deeltjes is mogelijk door het toevoegen van lagen tot een gewenste diameter. Dit is net als de groei van een sneeuwbal. De uitdaging is om deeltjes te maken die allemaal dezelfde maat hebben en die apart blijven tijdens de groei. Anders is het resultaat niet uniform, maar een mengsel van sneeuwpop-vormige deeltjes. Deze problemen zijn het ergst bij het maken van kleinere deeltjes $(<0.5 \mu \mathrm{m})$. Tegelijkertijd hebben deze als voordeel dat ze minder beïnvloed worden door de zwaartekracht (en zich dus meer als atomen gedragen). Met recente verbeteringen van microscopen wordt het mogelijk om kleinere deeltjes te zien. Doordat we deze deeltjes nu kunnen zien, is het de moeite waard geworden om de creatie ervan te verbeteren. In dit hoofdstuk overwonnen we problemen rond het maken van uniforme en kleine deeltjes. $\mathrm{Nu}$ zijn ze klaar voor gebruik als verbeterd atoommodel.

Hoofdstuk 3 houdt zich ook bezig met het maken van colloïdale deeltjes, maar hier in de vorm van vloeibare druppeltjes. Het is moeilijk om deze druppeltjes te maken met een uniforme diameter, vooral in de colloïdale maat. Een oplossing is om elke druppeltje apart te maken met zoiets als een micrometer druppelende kraan. Vloeistofbehandeling op deze kleine schaal heet microfluïdica. Deze methode, waarbij elke druppel apart wordt gemaakt, is langzaam in vergelijking met andere manieren om druppeltjes te maken. Om dit te versnellen onderzochten we in Hoofdstuk 3 een methode met 5000 'kraantjes' in 
parallel. Hierdoor konden we de productie van uniforme druppeltjes versnellen.

In het 'observatie' deel van het proefschrift, Hoofdstuk 4, gaat het over de kalibratie van een moderne lichtmicroscoop. Net als in alle wetenschappelijke beeldapparatuur, is het belangrijk om te controleren dat de microscoop de werkelijke dingen weergeeft. Voor deze meting gebruikt men een voorwerp dat even groot is als het kleinste ding dat gezien kan worden door de microscoop. Naast kalibratie, vergemakkelijkt deze meting ook het beeldherstel in nabewerking. Ter illustratie, stel je een camera voor die foto's maakt die allemaal verticaal vervaagd zijn (misschien is de sensor/film los en beweegt hij als de ontspanknop ingedrukt wordt). Als test maak je eerst een foto van een kleine of verre lichtbron (zoals een ster). Het zal in de weergave verschijnen als een verticale lijn, terwijl we weten dat het een puntje moet zijn. Gebaseerd op hoe uitgerekt het puntje is, weten we hoeveel vervaging plaatsvindt en hoeveel we moeten corrigeren. Als we dan vervolgens een foto van een gezicht maken, zal hij bijna onherkenbaar zijn door de vervaging. Maar het portret is gewoon een collectie van vele vervaagde puntjes, die we begrijpen dankzij de meting van de ster. Met deze kennis kunnen we de vervaging (gedeeltelijk) omkeren. Hierdoor kunnen meer details gezien worden in de herstelde foto, maar sommige informatie is onherstelbaar verloren.

De microscoop uit dit hoofdstuk kan 3 D volume afbeeldingen maken door foto's te maken van een plak per keer zoals in een medische CT scanner. Meestal wordt een plat $2 \mathrm{D}$ kalibratiebeeld gebruikt om microscopen te kalibreren, maar dit is niet voldoende voor een $3 \mathrm{D}$ microscoop. Daarom ontwikkelden we in dit hoofdstuk een dik testvoorwerp met de gewenste kleine puntjes (fluorescent silicadeeltjes) gedistribueerd in $3 \mathrm{D}$. De bijdrage van dit hoofdstuk was het maken en demonstreren van een voorwerp gemaakt van fluorescent colloïdale deeltjes dat geschikt is voor deze kalibratietesten.

Tot slot onderzochten we in Hoofdstuk 5 de manipulatie van colloïden. Deeltjes reageren op verschillen in zoutconcentratie door te bewegen van of naar regio's van hoge concentratie. Door de zoutconcentratie in hun omgevingen te beheersen, is het mogelijk om de snelheid en de richting van hun beweging te veranderen. De experimenten waren met deeltjes die door een microfluïdische kanaal stroomden, waarbij de zoutconcentratie in het midden anders was dan die van de zijkanten.

Om dit te illustreren, kan je denken aan een menigte die allemaal het treinstation verlaten op weg naar de stad. Mensen reageren nauwelijks op zout op straat, dus in plaats daarvan onderzoeken we hun reactie tot kauwgum op de grond. We zetten veel kauwgum op de stoep van de straat en niks in het midden. Doordat niemand vindt het leuk om op plakkerige gum te lopen, zullen de mensen onderweg naar de stad steeds meer in het midden van de straat gaan lopen. De deeltjes in dit hoofdstuk doen iets soortgelijks, hoewel 
ze meestal bewegen naar de regio met een hoge zoutconcentratie.

Dit fenomeen werd al onderzocht door te kijken naar het gemiddelde gedrag van veel deeltjes. In ons voorbeeld, is dit verwant aan onderzoek naar de mensen met het gebruik van alleen de overlappende voetafdrukken die zijn achtergelaten als de straat weer leeg is. Hiervan is de richting van beweging naar het midden duidelijk en een snelheid kan berekend worden van hoe ver van de stationsdeur iedereen gefocust in het midden van de straat wordt. Dit soort onderzoek zou het moeilijk vinden om een gedetailleerde theorie te testen. Bijvoorbeeld een theorie die voorspelt dat mensen bewegen met verschillende snelheden, op basis van de hoeveel kauwgum die op dat moment onder hun voeten is. In dit hoofdstuk zoomden wij in en analyseerden we de individuele deeltjes in het microfluïdische kanaal om zo de fijne details van een vergelijkbare theorie te bevestigen. 


\section{Acknowledgements}

Just as important as the unknowing giants whose shoulders I stand atop is the crowd of friends and colleagues who have carried me in times of need. To all those who have kindly lent their hand to help me along a little or a lot, I would like to extend my thanks.

First and foremost, I would like to thank my supervisor, Alfons van Blaaderen, for sharing his deep insight and great vision. I learned a great deal from you and have great respect for your pure attitude to science and egalitarian attitude to research teams.

I am grateful also to my reading committee for lending their time and expertise in the assessment of this thesis. Thank you Professors van Roij, Kegel, Voets, Mugele and de Groot.

The scientific staff of SCMB shared their expertise in many forms. Thank you Arnout, Gerhard, Marjolein, René, Joost, Laura, Freddy, Marijn, Hans, Patrick and Krassimir for sharing your wisdom over the years.

I had a lot of help at crucial times from the administrative support staff. Thank you Marion, Hester and Dianne for all your assistance and amazing capacity for getting things done.

More heroes may be found among the technical staff who made this work possible. Thank you Peter, Judith, Relinde, Elleke, Dave, Fabian, Chris and Hans for all your direct and indirect assistance.

I had the pleasure of supervising several enthusiastic and hard working students. Thank you Davide, Anan, Wendy and Roy, I learned something from all of you.

All of my thesis chapters were enriched by collaborations of one sort or another. Thank you Relinde, Judith, Corentin, Mathieu, Alessia, Jantina, Ernest, Pepijn, Rama, Doug, José, Sina, Da, Matthijs, Wessel, Fabian, Robin, Robin and Henriëtte.

I have shared my office office with a host of characters over the years. Thank you to Wiebke, Siddharth, Maarten, Stijn, Albert, Doug, Rama and Naveed for many tips and a pleasant working atmosphere. Thank you also to all the other members of SCM past and present who made my time here enjoyable and productive.

In my various flats in Utrecht, there was always a friendly physicist or two to be found. Thank you John, Simone (and sometimes Jessi), Giulia (and 
sometimes Guido) and Pepijn (and sometimes Christine).

I was lucky enough to enjoy many friendships during my PhD. Particular thanks are due to Giulia, Guido, Doug, Tonnishtha, Wiebke, Siddharth, Naveed, Rama, Anna, Simone, Jessi, Ernest, Zdenek, Carmine, Fara and Pepijn for all the fun in the good times and support when times were tough.

Thank you Mum, Dad and Duncan for all your support and thank you Granny for always being ready to save the date for my Nobel Prize ceremony.

Finally, thank you Eke for wading through several versions of my Dutch summary, for believing in me when I did not, and for bringing much joy into my life. 


\section{About the author}

Chris Kennedy was born on the $20^{\text {th }}$ of February 1993 in Glasgow, Scotland. He attended Strathaven Academy and was awarded the Dux Medal in 2009. The following year he commenced his undergraduate studies in chemistry and physics at the University of Glasgow. He was the top physics student in his third year. His fourth year was spent on exchange at Utrecht University where he carried out a research study on colloidal rod shaped particles under the supervision of Thijs Besseling and Arnout Imhof. Thereafter he returned to Glasgow where he completed his thesis project on remote focussing microscopy. In 2015, he graduated with a MSci in chemical physics (first class). Later that year he returned to Utrecht to begin his doctoral research with Alfons van Blaaderen, which is described in this thesis.

Chris first encountered the word 'colloid' in 2004 while reading Scorpia, a children's spy novel by Anthony Horowitz. Perhaps it was a childhood memory that led him to spend $£ 2$ on Colloid Science (Reference 72) in 2012, when Glasgow University Library was selling off some old textbooks. Since then, his interest has only deepened and with this thesis, he has produced his own little book about colloids. 